\title{
Organic Bioelectronics for In Vitro Systems
}

\author{
Charalampos Pitsalidis, Anna-Maria Pappa, Alexander J. Boys, Ying Fu, Chrysanthi-Maria Moysidou, \\ Douglas van Niekerk, Janire Saez, Achilleas Savva, Donata Iandolo, and Róisín M. Owens*
}

Cite This: https://doi.org/10.1021/acs.chemrev.1c00539

Read Online

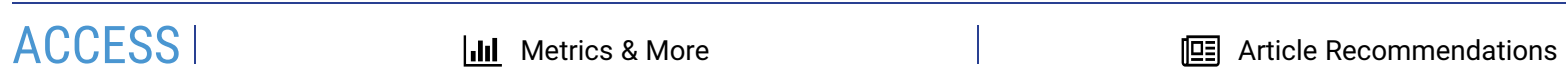

\begin{abstract}
Bioelectronics have made strides in improving clinical diagnostics and precision medicine. The potential of bioelectronics for bidirectional interfacing with biology through continuous, label-free monitoring on one side and precise control of biological activity on the other has extended their application scope to in vitro systems. The advent of microfluidics and the considerable advances in reliability and complexity of in vitro models promise to eventually significantly reduce or replace animal studies, currently the gold standard in drug discovery and toxicology testing. Bioelectronics are anticipated to play a major role in this transition offering a much needed technology to push forward the drug discovery paradigm. Organic electronic materials, notably conjugated polymers, having demonstrated technological maturity in fields such as solar cells and light emitting diodes given their outstanding characteristics and versatility in processing, are the obvious route forward for bioelectronics due to their biomimetic nature, among other merits. This review highlights the advances in conjugated polymers for interfacing with biological tissue in vitro, aiming ultimately to develop next generation in vitro systems. We showcase in vitro interfacing across multiple length scales, involving biological models of varying complexity, from cell components to complex 3D cell cultures. The state of the art, the possibilities, and the challenges of conjugated polymers toward clinical translation of in vitro systems are also discussed throughout.
\end{abstract}

\section{CONTENTS}

1. Introduction

1.1. Market Drivers, Challenges, and Opportunities

1.2. In Vitro Systems: Improving Authenticity and Reliability of the Model

1.3. Toward in Vitro Organic Bioelectronics

2. Organic Electronic Materials and Devices to Interface with Biological Systems

2.1. Materials for In Vitro Organic Bioelectronic Systems

2.1.1. Electronic Properties of CPs

2.1.2. Requirements of CPs for In Vitro Applications

2.1.3. Doped Conjugated Polymers: The Case of PEDOT:PSS

2.1.4. Undoped Conjugated Polymers

2.2. Organic Bioelectronic Interfaces

2.2.1. Surface Functionalization

2.2.2. Surface Topography

2.2.3. Tailoring Biointerfaces via Redox Switching

2.3. Organic Bioelectronic Devices

2.3.1. Electrodes

2.3.2. Transistors

2.3.3. Ion Pumps

2.3.4. Photoactuators
2.4. Modeling Organic Bioelectronic Devices for In Vitro Applications

2.4.1. Analytical Modeling

2.4.2. Empirical Modeling In Vitro

3. Interfacing Organic Bioelectronics with Cells

3.1. Cell Membrane Models

3.1.1. Toward Native Cell Membrane Models

3.2. 2D Cell Cultures

3.2.1. Electrogenic Cells

3.2.2. Nonelectrogenic Cells

3.2.3. Nonmammalian Cells

3.3. 3D Cell Cultures

3.3.1. Tissue Engineering Approaches

3.4. Stimulation Applications

3.4.1. Stimulation of Electrogenic Cells

3.4.2. Stem Cells

4. Monitoring Biomarkers in Cell Cultures

4.1. Biomarkers Relevant to Cell Activity

4.2. Organic Electronics for Cell-Based Biosensing

U

$\mathrm{V}$

W

$Y$

$Y$

AC

AD

AD

Al

$\mathrm{AO}$

$\mathrm{AQ}$

AR

$A Z$

$A Z$

$\mathrm{BB}$

$B F$

BF

BG 
DATA ACQUISITION, MODELLING AND ANALYSIS

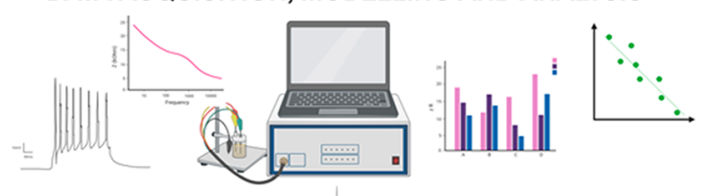

DEVICES

PLATFORMS

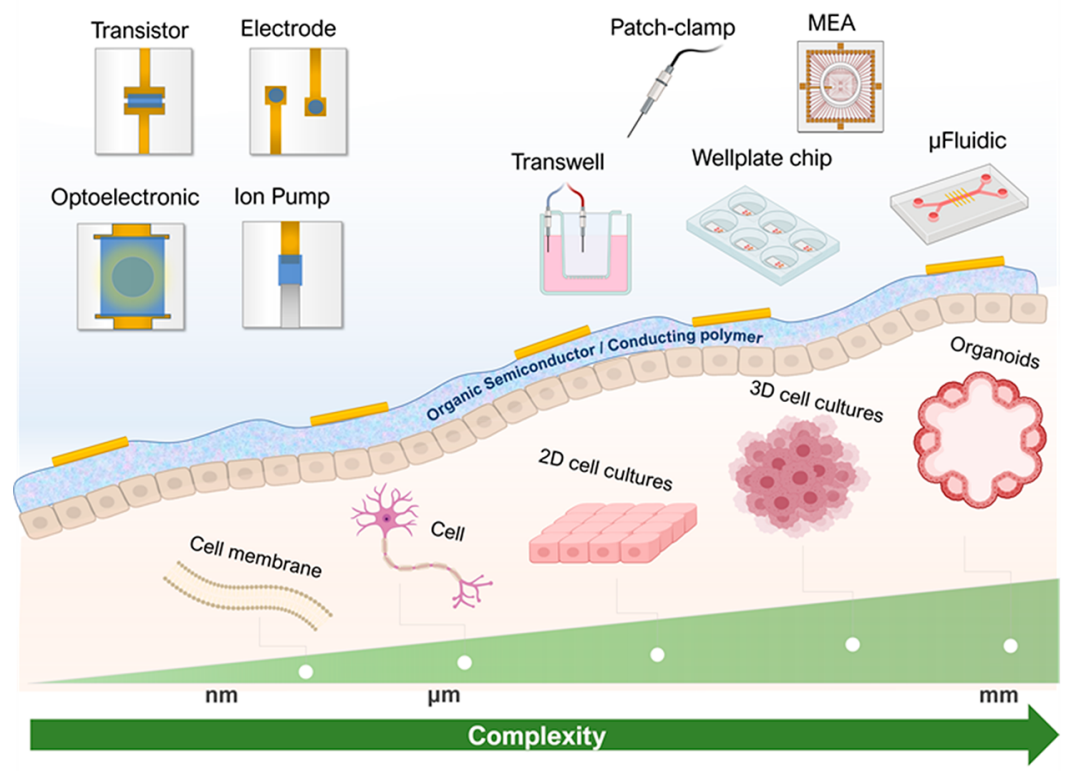

Figure 1. Schematic overview of in vitro organic bioelectronics showing the biointerface with state-of-the-art electronic devices and platforms used for monitoring and controlling function and activity of biological systems of increasing complexity. Created with BioRender.com.

$\begin{array}{lr}\text { 4.3. State of The Art of Cell-Based Biosensors } & \text { BH } \\ \text { 4.3.1. Sensing Secreted Metabolites from Cells } & \text { BH } \\ \text { 4.3.2. Sensing Cell Surface Biomarkers } & \text { BI } \\ \text { 4.4. Characteristics and Challenges of Cell-Based } & \\ \text { Biosensors } & \text { BK } \\ \text { 4.4.1. Cell culture: Biosensor Positioning } & \text { BK } \\ \text { 4.4.2. Eliminating the Interference } & \text { BK } \\ \text { 4.4.3. Improving Device Performance } & \text { BK } \\ \text { 5. On-Chip or Fluidic-Integrated In Vitro Models } & \text { BK } \\ \text { 6. Perspectives } & \text { BN } \\ \text { 6.1. Technological Challenges To Be Overcome } & \text { BN } \\ \text { 6.2. Opportunities } & \text { BN } \\ \text { Author Information } & \text { BO } \\ \text { Corresponding Author } & \text { BO } \\ \text { Authors } & \text { BO } \\ \text { Notes } & \text { BO } \\ \text { Biographies } & \text { BO } \\ \text { Acknowledgments } & \text { BP } \\ \text { References } & \text { BP }\end{array}$

\section{INTRODUCTION}

Cell-based in vitro models (including models of the cell membrane) have been increasingly adopted for applications ranging from tissue engineering to drug discovery and toxicology. ${ }^{1}$ In addition, a significant body of work on in vitro models aims at simulating biological reactions to materials for later use in vivo (or in the body) as well as to survey newly developed materials. In brief, compared to in vivo studies, in vitro studies, apart from being ethically advantageous, are faster and more cost-effective and can be easily standardized and validated. ${ }^{2,3}$ Advances in $3 \mathrm{D}$ cell cultures as well as the advent of microfluidics ${ }^{4}$ and the downsizing of large-scale biological assays ${ }^{4}$ heralded a new era of in vitro models, ${ }^{5,6}$ amplifying the role such studies will play not only for gaining insight into basic biological systems, but also in the early stages of development and testing of therapeutic compounds. However, there remain challenges related mainly to the authenticity and validity of those systems as well as the lack of a standardized and adaptable technology for meaningfully converting biological signals to a readable output. ${ }^{7}$ Electrochemical readout schemes can be highly advantageous for the assessment and standardization of in vitro platforms providing a wealth of real-time information given that they are direct, more adaptable, and more scalable compared to optical methods, currently dominating biological assays. ${ }^{8}$ Within this realm, organic electronics are anticipated to play a key role for biointerfacing, bridging the gap between the biotic and the abiotic. This review covers the latest advances in the use of organic electronic devices to interface, monitor, and control in vitro systems of varying degrees of complexity, from the simplest protein-ligand interactions to cell membrane models to complex 3D cell cultures. Ultimately, generation of authentic biological models interfaced with bespoke electronic devices will lead to more reliable and efficient biological assays (see Figure 1).

Section 1 provides an overview of in vitro systems, the state of the art, the market, and the challenges currently faced. We also discuss how (organic) bioelectronics can advance this field by providing technological solutions to existing bottlenecks. The in vitro systems covered are cell-based systems (including isolated cell membranes), excluding studies based on molecules purified from cells (such as proteins and nucleic acids), which focus on 
detection outside of the cell context. The fundamental aspects of the conjugated polymers (section 2.1 ), the in vitro organic bioelectronic interfaces (section 2.2), and the resulting device architectures (section 2.3) are covered in section 2. We do not cover small molecule organic electronics and carbon-based materials, including carbon nanotubes (CNTs) and graphene. The core section of the review, section 3 , covers the state of the art in organic bioelectronic cell-based in vitro systems, where organic electronics technology is used for monitoring and transducing biological activity. The categorization we follow is based on the complexity of the biological model of study, starting from the simplest cell membrane model (section 3.1), followed by typical $2 \mathrm{D}$ cell cultures or cell monolayers (section 3.2 ), then more advanced $3 \mathrm{D}$ or organotypic cultures (section 3.3 ), and finally the integration of microfluidics toward miniaturized "organ" on-chip systems (section 3.4). The last part of this section gives an overview of the different modeling and analysis methodologies that have been used to date to translate the generated electrical signals to meaningful biological properties. Section 4 summarizes the state-of-the-art in the use of organic bioelectronics technologies for controlling or stimulating cell cultures. Section 5 provides an overview of the use of organic electronic sensors for monitoring biomarkers from or in cells. This section covers applications where the sensors are used in the context of cell-based in vitro systems, hence detecting biomarkers in cell cultures, either in-line or remotely. Sensors for proteins or nucleic acids from diverse biofluids are excluded from this review. The last section of the review, section 6 discusses the challenges and the perspectives of this field for the future of in vitro systems.

\subsection{Market Drivers, Challenges, and Opportunities}

In recent years, there has been a marked decline in the number of approved therapeutics, with attrition rates in drug discovery increasing at an alarming rate. ${ }^{9}$ In addition, tighter safety regulations result in increasing development costs and decreasing profitability of new medicines, associated with the high costs of animal studies and their failure to predict adverse effects of promising drug candidates. ${ }^{10}$ Two key areas expected to improve success rates are (i) the discovery of new biomarkers and more specific drug targets driven by advances in precision medicine $\mathrm{e}^{11-13}$ and (ii) the availability of preclinical models that better recapitulate thein vivo biology and microenvironmental factors while enabling the assessment of thousands of compounds. ${ }^{14}$ Animal models, the gold standard for assessing the potency and toxicity of pharmaceutical compounds, not only pose limitations in terms of throughput capabilities but also suffer inherent differences from human physiology and have frequently failed to predict the fate of a given drug. ${ }^{15,16}$ The use of accurate and reliable in vitro models at appropriate points during drug development could greatly improve the chances of identifying successful drug candidates.

There is now a push toward improving predictability of in vitro models, thus providing a potential replacement for animal experiments, in line with the "three Rs" (reduction, refinement, replacement). ${ }^{17}$ Looking at the in vitro toxicology market, which is expected to reach USD 14.9 billion by 2025 from an estimated USD 9.1 billion in 2020, at a CAGR (Compound Annual Growth Rate) of $10.3 \%$, growth in this market is primarily driven by technological advancements to detect toxicity at an early stage during drug development, overcoming the use of animal testing. ${ }^{18}$ According to the same report, the cell culture technologies segment accounts for the largest share of the in vitro toxicology technologies market. Similarly, looking at cellculture assays, this market is projected to reach USD 22 billion by 2025 from USD 14.9 billion in 2020 , at a CAGR of $8.1 \%{ }^{19}$ The rising need for cell-based assays in drug discovery has resulted in ever-increasing funding for cell-based research, and growth in the number of drug discovery activities are the key factors supporting the growth of the market. It should be noted that the $3 \mathrm{D}$ cell culture market contributes to the broader drug discovery and tissue engineering market. Specifically, organ-onchip (OoC) technologies have undergone significant growth over the last 5 years as they offer great promise for creating powerful preclinical models and are expected to play a pivotal role in accelerating drug discovery and development. This provides tremendous opportunity for new market players to enter this field. Current trends for organs-on-chips reveal a market size of USD 303.6 million by 2026 at a CAGR of $39.9 \% .^{20}$

\subsection{In Vitro Systems: Improving Authenticity and Reliability} of the Model

In vitro (within glass, originally denoting a glass test tube or Petri dish) studies are typically performed with microorganisms, cells, or biological molecules outside of a living organism. Typical examples of in vitro studies include the isolation and growth of cells derived from multicellular organisms including the use of subcellular components or extracts, as well as molecules purified from cells. The key challenge for cell-based in vitro models is to mimic, as accurately as possible, the in vivo state of the biological system under study. It is also essential that the limitations of the model are understood. The integration of electrical transducers offers an opportunity to noninvasively interface with these biological models as we will detail here.

The traditional method to study cells outside their natural environment, developed in the early 1900s, is in twodimensional (2D) cell cultures (or cell monolayers). 2D in vitro systems represent an oversimplification of the in vivo situation since, inherently, they fail to mimic the necessary cellcell and environmental conditions responsible for cell differentiation, proliferation, and viability, required for functional tissues and organs. ${ }^{21,22}$ In the past $10-15$ years, advances in $3 \mathrm{D}$ cell biology and stem cell research have brought to the fore novel and robust biological tools or bricks for building more representative and reliable in vitro systems. ${ }^{14,23,24}$ Importantly, the discovery of induced pluripotent stem cells (iPSCs) led to another major breakthrough, heralding a new era in the field of biomedical research. ${ }^{14,25}$ Other progress paths involve the use of scaffolding materials (i.e., hydrogels, fibers) for 3D cell culture in fluidics chips ${ }^{26}$ and the incorporation of inline sensors that can be easily standardized and give quantifiable information in simple readout formats.

\subsection{Toward in Vitro Organic Bioelectronics}

Traditionally, electronic assays have been used for the study of electroactive cells. The most prevalent method of assessing neuronal activity has historically been patch-clamp electrophysiology and, more recently, (high-density) planar microelectrode arrays (MEAs), for monitoring intracellular or extracellular and mainly extracellular activity, respectively. ${ }^{27} \mathrm{It}$ is now widely accepted that the electrical properties of nonelectroactive cells are relevant to their natural function and can provide insight on complex physiological states of the cell or the tissue. ${ }^{28,29}$ Conventional (inorganic) electronic materials including gold, platinum, silicon, etc., benefiting from advances in the integrated circuit industry, have been successfully 
a

d
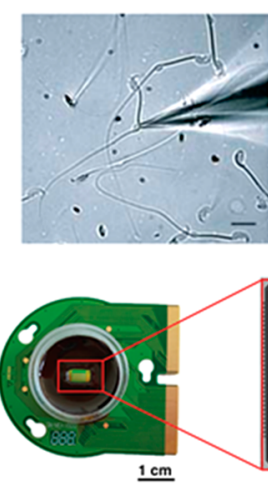

e

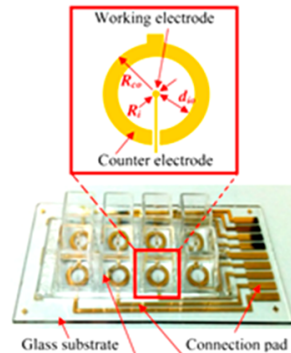

Plastic cell culture wells Connection lead

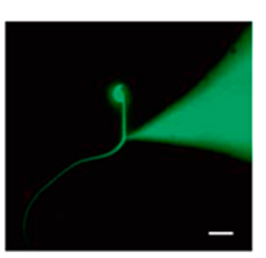

b
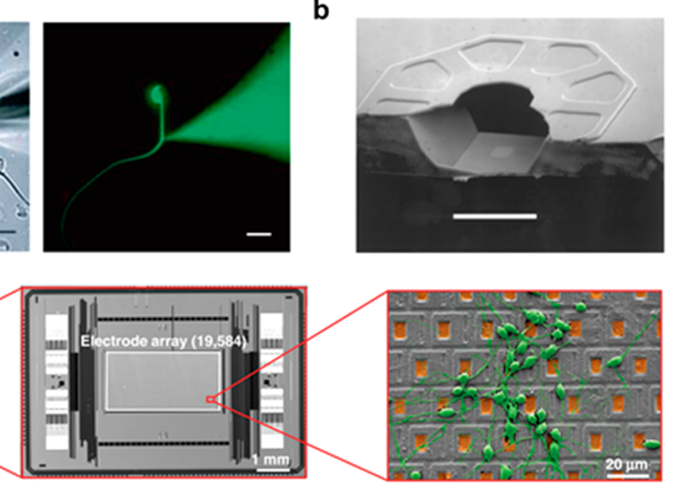

f

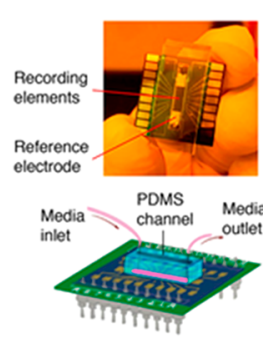

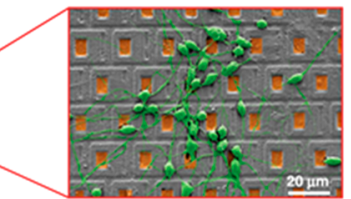

g

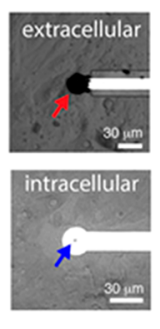

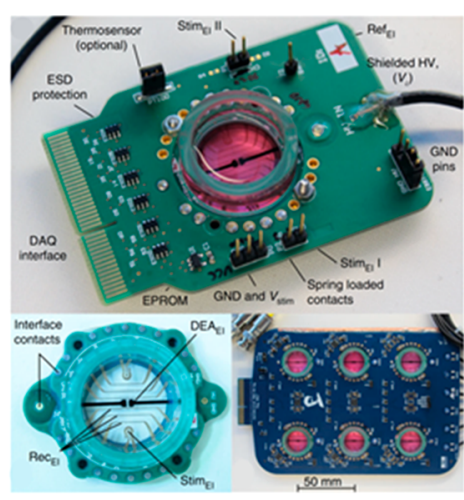

h
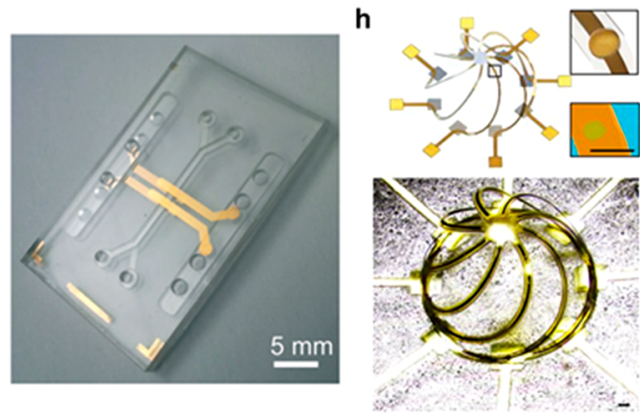

Figure 2. Inorganic in vitro bioelectronic systems. (a) Patch clamp recording process. Reproduced from ref 42 . Copyright 2014 Springer Nature under Creative Commons Attribution 4.0 International (CC BY 4.0) License, https://creativecommons.org/licenses/by/4.0/. (b) Neurochip, a multielectrode device for simultaneous stimulation and monitoring. Adapted with permission from ref 43. Copyright 1999 Elsevier. (c) Mechanically active multielectrode array for generating strains and performing electrophysiological recordings in cardiac tissue. Reproduced from ref 44 . Copyright 2019 Springer Nature under Creative Commons Attribution 4.0 International (CC BY 4.0) License, https://creativecommons.org/licenses/by/4.0/. (d) Dual mode high density MEA platform capable of simultaneous monitoring from 19584 recording sites and 246 high signal-to-noise ratio channels. Reproduced from ref 45. Copyright 2020 Springer Nature under Creative Commons Attribution 4.0 International (CC BY 4.0) License, https://creativecommons.org/licenses/by/4.0/. (e) Electric cell-substrate impedance sensing (ECIS) chip with eight ECIS sensors. Adapted with permission from ref 46. Copyright 2017 Elsevier. (f) Microfluidic heart-on-a-chip model with integrated extracellular and intracellular bioelectronic elements for monitoring cardiac electrophysiology under acute hypoxia. Adapted with permission from ref 47 . Copyright 2020 American Chemical Society. (g) Microfluidic gut-on-a-chip device with integrated gold electrodes for TEER measurements under flow conditions. Reproduced with permission from ref 48. Copyright 2019 Royal Society of Chemistry under Creative Commons Attribution-NonCommercial 3.0 Unported License, https://creativecommons.org/licenses/by-nc/3.0/. (h) Schematic and photograph of a 3D electronic scaffold configuration used for stimulation and recording of dorsal root ganglion neurons. Reproduced from ref 49. Copyright 2017 PNAS under Creative Commons Attribution-NonCommercialNoDerivatives License 4.0 (CC BY-NC-ND) https://creativecommons.org/licenses/by-nc-nd/4.0/.

employed for in vitro biointerfacing in a variety of different scenarios with key examples being highlighted in Figure 2. A significant body of work exists on the integration of electrical transducers in cell-based models, employing electrodes, transistors, MEAs, and other devices, combined with electrical modeling and simulation, for real-time monitoring of biological events in cell culture. ${ }^{30-35}$ Electrochemical impedance spectroscopy (EIS) is a typical method used to assess the function and growth of cells in a noninvasive manner, ${ }^{36,37}$ and more recently, electrical impedance tomography (EIT) has shown potential to provide better spatial resolution in $3 \mathrm{D}$, offering more in-depth information within complex $3 \mathrm{D}$ cell systems. ${ }^{38}$ Although the aforementioned methods provide a solution to overcome the challenges in monitoring and characterizing $3 \mathrm{D}$ cell systems, most of the approaches rely on traditional, flat, rigid metal electrodes that are designed for $2 \mathrm{D}$ cell models. In addition, conventional electronic materials sometimes fail to establish intimate cell-electrode coupling that is necessary to accurately record a signal, limiting the type and depth of biological information that could be acquired notably in $3 \mathrm{D}$ biological systems. ${ }^{39-41}$

In contrast, organic electronic materials, being easily adopted in various forms, have been shown to more seamlessly integrate with biological systems of advanced complexity, providing better cell-electrode coupling and thus more effective signal transduction of biological events. ${ }^{39,41,50-53}$ Organic electronic materials including conjugated small molecules ${ }^{54}$ and polymers, ${ }^{55}$ exhibit mechanical compliance, negligible cytotoxicity and ease of functionalization and modification of the material surface (or bulk). Notably, conjugated polymers (CPs) are mixed conductors (ions and electrons), facilitating their direct coupling with biological systems, where ions are the predominant carriers, via a variety of different device designs.

Figure 3 captures our interpretation of the parameter space for interfacing organic electronic devices with in vitro models. Typically, this interfacing has been thought of as two-sided: stimulation on the one hand and monitoring on the other. We introduce a third component, which is the interfacing of biological systems with electronics, where the chemical or physical characteristics of the active layer of the device can alter the biological system being studied. Interfacing can be a powerful means of controlling biological systems when used carefully. In terms of models, the degree of complexity of an in vitro model can vary from isolated parts of the cell (i.e., cell membranes) (see section 3.1) to cell monolayer models (see section 3.2 ) to complex $3 \mathrm{D}$ (or organotypic) cell cultures (see 


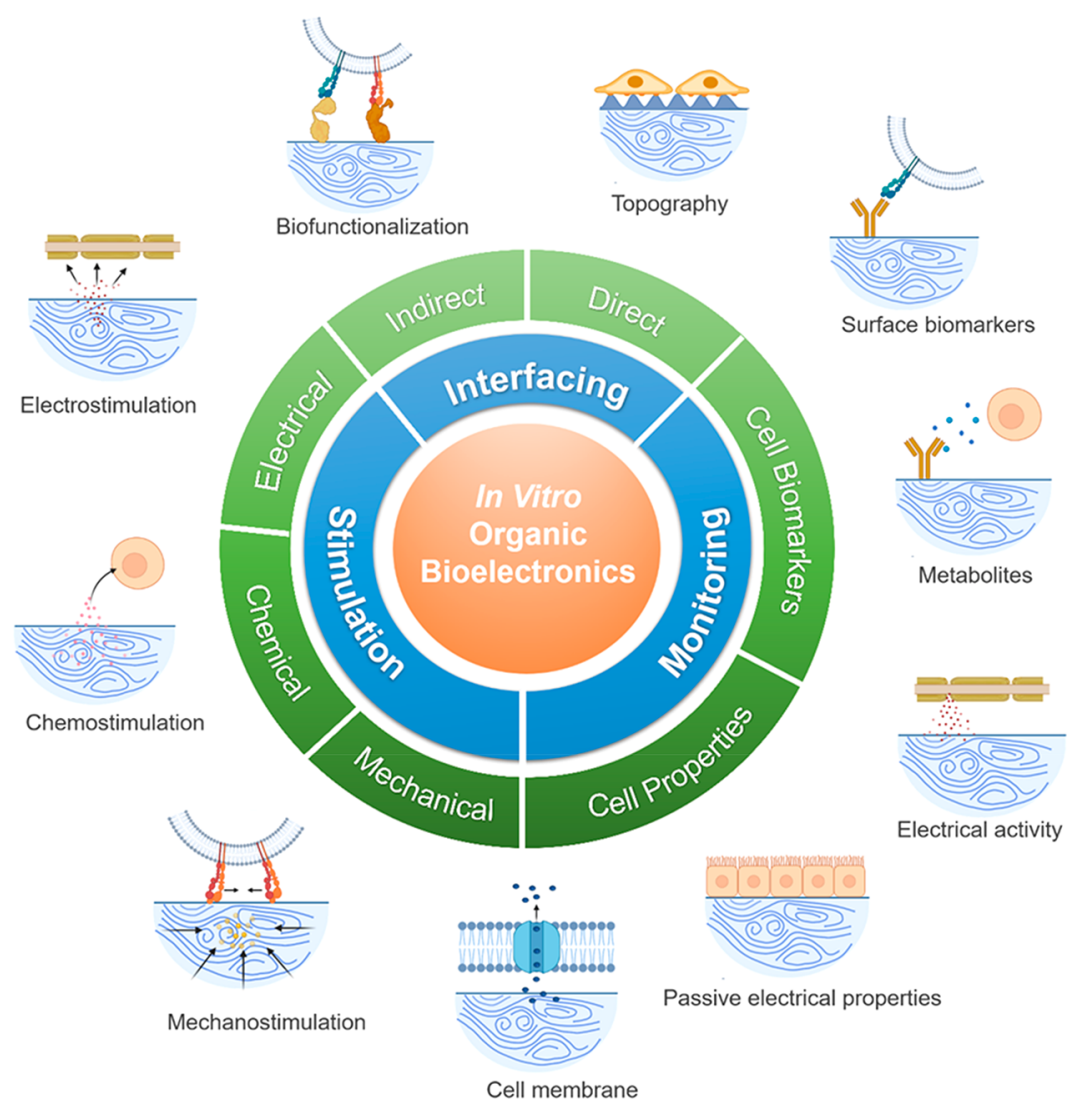

Figure 3. Schematic showing the three key functionalities of in vitro organic bioelectronics and the various associated configurations found at the conjugated polymer/cell junction. Created with BioRender.com.

section 3.3). Depending on the model under investigation and the intended application, different types of bioelectronic sensors are developed. Typically, electrophysiology sensors (i.e., sensors of cell electrical activity) operate via the transduction of the local membrane potential state as a function of time, by translating the ionic mass transfer into the electronic domain. ${ }^{56-58}$ Beyond traditional electrophysiology recordings of neurons and cardiac cells, electrical measurements are widely used to assess passive electrical properties of cells. For example, EIS, that is, the application of a frequency sweep of current, offers a straightforward and rapid way to monitor changes in the electrode impedance due to fundamental biological processes, such as cell adhesion and coverage, as well as a means to quantitatively characterize the integrity of barrier tissues (or cell membranes), by means of their electrical resistance, in a noninvasive manner. ${ }^{28,36,37,59,60}$

Sensors of cellular biomarkers, involving an analyterecognition unit, can be also interfaced with in vitro cultures to monitor secreted metabolites or surface biomarkers, resulting in biological interactions, which can be either Faradaic or electrostatic. $^{50}$ In the case of the former, the analyte binding event causes charge transfer between the CP and the analyterecognition unit, while in the latter, the binding event induces conformational changes, which modulate either the CP or the interface between the recognition unit and the CP (as detailed in section 4). ${ }^{29}$

In terms of stimulation, applications can be subdivided into mechanical, electrical and chemical stimulation, where in each case an actuation of the biological system alters the current state of the system, for example, inducing cell differentiation and migration $^{61,63,64}$ (see section 3.4). Electrical stimulation refers to the application of an external electrical stimulus that can activate intracellular signaling and influence the intracellular microenvironment. ${ }^{65}$ As stated in the recent review by Gelmi and Schutt, ${ }^{66}$ the electroactive material delivers the stimulus to cells either via an electric field or via charge injection. The role played by the electrically responsive biomaterial in signal transduction is typically direct charge injection into the biological entity. Chemostimulation involves modulating the exposure of a biological system to controlled amounts of chemical species or drugs (i.e., growth factors), which can result in the doping and dedoping of CPs. ${ }^{62}$ Further, alterations in the volume of the electroactive material, as a result of doping and dedoping state of a CP, could induce a mechanical strain on a biological entity should it be bound to the functionalized surface of the material. Finally, interfaces allow for both measurement and actuation by controlling the coupling between biological and electronic systems by means of surface functionalization or patterning (see section 2.2)..$^{61,62}$

\section{ORGANIC ELECTRONIC MATERIALS AND DEVICES TO INTERFACE WITH BIOLOGICAL SYSTEMS}

\subsection{Materials for In Vitro Organic Bioelectronic Systems}

Conjugated polymers alone or in combination with other organic materials have been extensively used to interface with biological systems in vitro. ${ }^{67,64}$ Almost all CPs used for in vitro applications are soluble in common solvents and allow facile solution processing in different formats. CPs have been explored as thin film coatings on flat rigid or flexible $2 \mathrm{D}$ interfaces ${ }^{68}$ and as electroactive $3 \mathrm{D}$ interfaces for growing and monitoring cell cultures. ${ }^{51}$ Notably, CP properties can be tuned at the molecular 


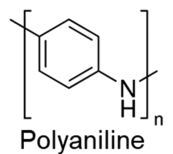

(PANi)

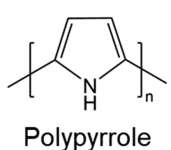

(PPy)

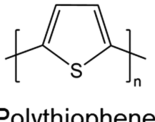

(PTh)

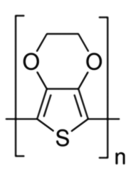

Poly(3,4-ethylenedioxythiophene) (PEDOT)

Figure 4. Chemical structure of the most commonly used conjugated polymers for in vitro bioelectronics applications.

level through chemical synthesis or via other routes enabling de novo design of materials that are tailor-made for biological applications highlighting their unprecedented potential for bioelectronics. $^{53}$

2.1.1. Electronic Properties of CPs. CPs comprise a backbone of alternating double and single bonds with overlapping $\pi$-orbitals that create a system of delocalized $\pi$ electrons. ${ }^{69}$ This delocalized system can result in electrical conductivity values several orders of magnitude higher than insulating polymers ${ }^{70}$ (i.e., $>10^{-9} \mathrm{~S} \mathrm{~cm}^{-1}$ ) and strong optical absorption in the visible spectrum of light. ${ }^{71}$ The most commonly used CPs in bioelectronics are shown in Figure 4. Polyaniline (PANI) and polypyrrole (PPy) represent two of the very first CPs reported to exhibit semiconducting properties. $^{72,73}$ Polythiophenes (PTh) and derivatives, including poly(3,4-ethylene dioxythiophene) (PEDOT), have been extensively interfaced with cell cultures in vitro. The aforementioned CPs behave as semiconductors and hence in their neutral state exhibit low electrical conductivity in the range of $10^{-7}$ to $10^{-3} \mathrm{~S} / \mathrm{cm}$. When semiconducting CPs are chemically doped, ${ }^{74,75}$ they can form stable materials with metal-like conductivity (above $1 \mathrm{~S} / \mathrm{cm}$ and up to several $\mathrm{kS} / \mathrm{cm}$ ). These materials are known as (intrinsically) conducting polymers. So far, both conducting and semiconducting polymers have been used for in vitro bioelectronics.

2.1.2. Requirements of CPs for In Vitro Applications. Direct contact with biological tissue poses specific complications, and therefore, the set of requirements that CPs need to meet are quite demanding in order to noninvasively exchange electrochemical signals (i.e., without damaging each other). Figure 5 summarizes the critical material properties that in vitro bioelectronic interfaces require.

2.1.2.1. Biocompatibility. CPs must be biocompatible to avoid injurious effects to biological systems. Most CPs are inherently biocompatible because they are mainly made of chemical elements that match the organic composition of cells and tissue, such as carbon and hydrogen. However, biocompatibility of CPs cannot be universally defined because they can elicit different biological responses depending on the type of cells and the local tissue environment. ${ }^{79}$ Moreover, CPs are often modified with functional groups or mixed with additives, which could be harmful for the in vitro system of interest. An example of a healthy cell culture growing within a CP-based mesoporous scaffold is shown in the top left image ${ }^{40}$ of Figure 5.

2.1.2.2. Stability. Another critical requirement is the stable operation of these materials in biologically relevant electrolytes or cell culture media. Material composites based on CPs that are used for this purpose are usually water-soluble, and strategies have been employed to avoid deformation or delamination upon immersion in physiologically relevant electrolytes. ${ }^{80}$ The electrical output of the materials must remain stable for many cycles of operation to guarantee accurate electrical monitoring of biological events. ${ }^{81}$ An example is shown in the top right image of Figure 5, where the current output of the organic

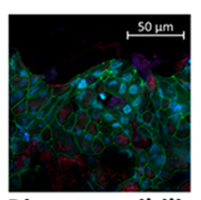

Biocompatibility
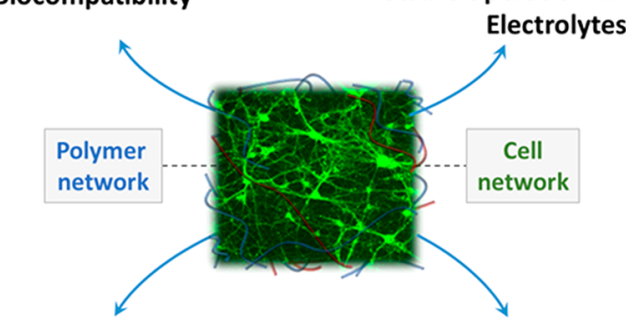

Ionic and Electronic Conduction
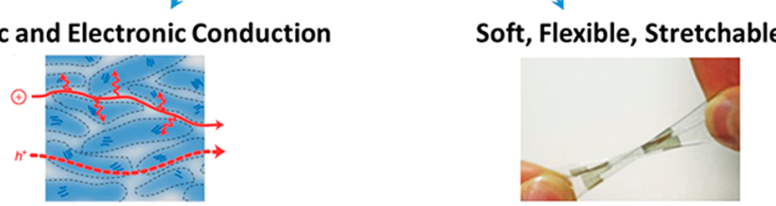

Figure 5. Critical properties required by CP-based materials to be used as bioelectronic interfaces for in vitro bioelectronics. Biocompatibility. Adapted with permission from ref 40. Copyright 2021 The Authors. Advanced Biology published by Wiley-VCH GmbH under the terms of the Creative Commons CC BY license, https://creativecommons.org/ licenses/. Stable operation in aqueous electrolytes. Reproduced with permission from ref 76. Copyright 2020 The Authors. Published by WILEY-VCH Verlag GmbH \& Co. KGaA, Weinheim, under the terms of the Creative Commons CC BY license https://creativecommons. org/licenses/. Mixed ionic and electronic conduction. Adapted with permission from ref 77. Copyright 2016 The Authors. Published by Springer Nature under a Creative Commons Attribution 4.0 International License http://creativecommons.org/licenses/by/4.0/. Soft, flexible, strechtable. Adapted from ref 78. Copyright 2019 WILEYVCH Verlag GmbH \& Co. KGaA, Weinheim.

bioelectronic device is tested for 1000 on/off cycles and the current output remains unchanged. ${ }^{76}$

2.1.2.3. Mixed Conduction. One of the most important properties of CPs that makes them increasingly popular for bioelectronic applications is their ability to conduct both ionic and electronic currents. ${ }^{82}$ When electrically addressed (biased) in aqueous electrolytes, ions migrate from the electrolyte into the bulk of the polymer to compensate for electronic charges. ${ }^{83}$ As such, ionic fluxes originating from biological activity can directly alter the electronic properties of CPs when in contact with cells or tissue. ${ }^{84}$

2.1.2.4. Mechanical properties. Mechanical properties of CPs are also crucial for in vitro interfacing. For example, their literal flexibility enables their adaptation to various form factors and can be tuned on demand to serve the requirements of a given biological model. Their typically low mechanical stiffness, indicated by the material Young's modulus, is a critical parameter, especially for complex tissue cultures. Biological tissues exhibit a wide range of mechanical stiffness, spanning multiple orders of magnitude (i.e., $\mathrm{kPa}$ for brain, $\mathrm{MPa}$ for 

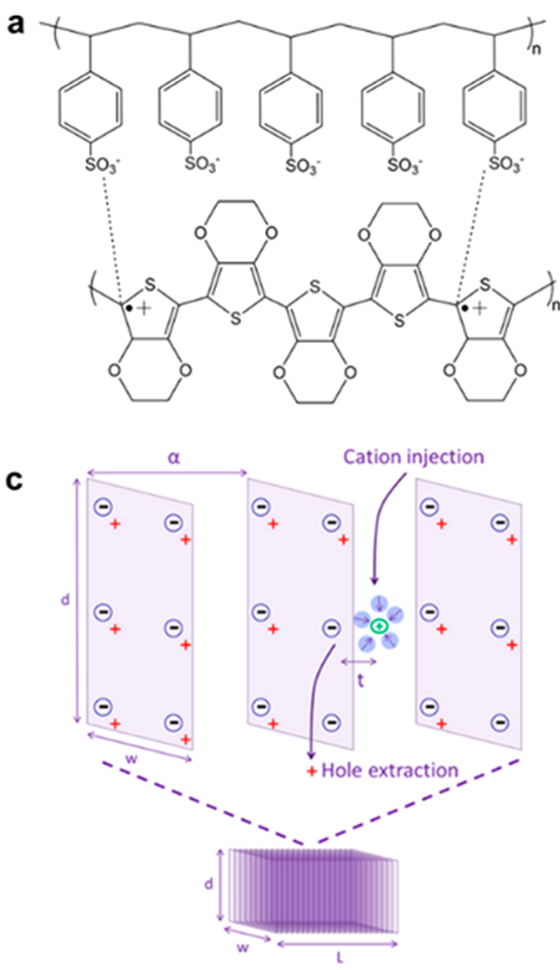

d
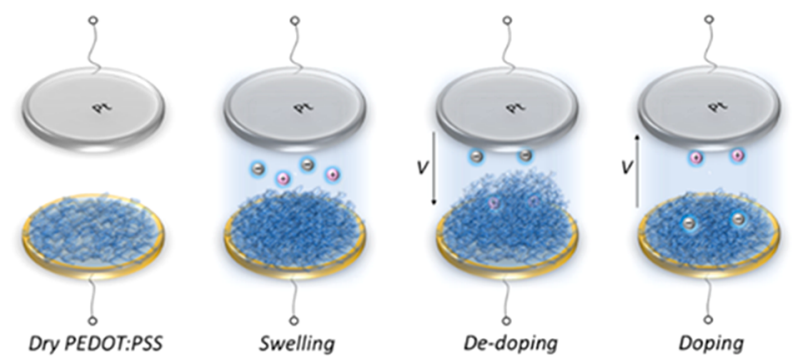

Figure 6. (a) Chemical structure of PEDOT:PSS. (b) Schematic showing the dedoping of PEDOT:PSS with cations from an electrolyte. (c) Schematic showing an array of plates against which ions pile up to form double layer capacitors. Reproduced with permission from ref 106 . Copyright 2016 John Wiley and Sons. (d) Reversible swelling of PEDOT:PSS film when in contact with the electrolyte and during dedoping/doping cycles due to up-take of cations and water. Adapted with permission from ref 112. Copyright 2018 The Authors, published Royal Society of Chemistry under a Creative Commons Attribution 3.0 Unported License, https://creativecommons.org/licenses/by/3.0/.

muscle, GPa for bone). ${ }^{85}$ Given that most CPs show a Young's modulus in the range of $\sim 1 \mathrm{GPa}^{86}$ (still significantly lower than silicon-based electronic materials), mixing with additives or other materials can adjust it to match the mechanical properties of the tissue of interest. ${ }^{87}$ The ability to match the mechanical properties of tissues renders CPs particularly attractive. CPs have been mixed with other functional materials or biopolymers to form multifunctional composites, including conducting hydrogels with low Young's modulus suitable for ultrasoft tissue. $^{88} \mathrm{~A}$ wide range of $\mathrm{CP}$-based ternary or quaternary multifunctional composites have been realized with tunable conductivity, low Young's modulus, self-healing properties, and biodegradability. ${ }^{89}$ These materials are ideal for soft electronic devices and tissue engineering. ${ }^{90}$ Bioderived materials like chitosan, ${ }^{91}$ hyaluronic acid, ${ }^{92}$ gelatin, ${ }^{93}$ and others have been mixed with CPs to form multifunctional interfaces for bioelectronic applications including wound healing ${ }^{94}$ and tissue engineering. ${ }^{95}$ PPy has been mixed or modified with functional groups and biomolecules for improving biocompatibility. ${ }^{96}$ Yang et al. used a conducting hydrogel based on PPy and hyaluronic acid with electrical conductivity of $7.3 \mathrm{mS} / \mathrm{cm}$ and low Young's modulus $(\sim 3 \mathrm{kPa})$ to culture $3 \mathrm{~T} 3$ cells in vitro. ${ }^{97}$ In addition, PPy doped with $p$-toluene sulfonate and incorporating the growth factor neurotrophin-3 has been synthesized and used to stimulate the outgrowth of auditory neuron explant cultures. $^{98}$ Furthermore, conducting 3D hydrogel matrices based on PPy mixtures with hyaluronic acid and carbon nanotubes were used to electrically stimulate the differentiation of human neural stem or progenitor cells. ${ }^{99}$

2.1.3. Doped Conjugated Polymers: The Case of PEDOT:PSS. PEDOT doped with the polyanionic dopant poly(styrene sulfonate) (PSS) is widely used in bioelectronics due to its stability in cell culture conditions, ${ }^{68}$ commercial availability, and excellent mixed ionic and electronic conduction properties. ${ }^{100,101}$ PEDOT:PSS is separated in two distinct phases: PEDOT is the p-type semiconducting polymer backbone that conducts holes in the form of polarons (or bipolarons), ${ }^{102}$ which are electrostatically stabilized by the negatively charged sulfonate groups of PSS (Figure 6a). The hydrophilic nature of PSS promotes good ionic mobility within PEDOT:PSS. ${ }^{103}$ When electrically addressed, in aqueous electrolytes, hydrated cations dedope PEDOT:PSS by disrupting the electrostatic polaron- $\mathrm{SO}_{3}{ }^{-}$bonds between the PEDOT backbone and PSS, respectively. ${ }^{104}$ As shown in the schematic of Figure 6b, PEDOT chains are then distorted, the hole transport pathways are disrupted, and therefore, the hole conductivity of the material is reduced. The conductivity modulation is the principle of operation for PEDOT:PSS-based bioelectronic devices. The dedoping mechanisms in PEDOT:PSS have been extensively investigated by several groups. ${ }^{77,105,106}$ Berggren and Malliaras recently described the elementary steps of this process, in terms of a PEDOT:PSS-coated metal electrode bathed in an electrolyte. ${ }^{107}$ First, a hole is injected from the metal electrode into the HOMO of the polymer. Second, the hole is transported within the delocalized HOMO of the polymer. Third, ions are injected from the electrolyte into the polymer. Fourth the ions are transported within the free volume between the polymer crystallites or chains, and fifth an electrostatic compensation of the two charges occurs in the bulk of the film. Romele et al. provided experimental evidence of the electrostatic compensation of the fixed charges in the bulk of PEDOT:PSS films by the mobile ions provided by the electrolyte. ${ }^{108}$ The bulk interaction 
a<smiles>CO[Si](C)(CCOCC1CO1)OC</smiles><smiles>CCOCCOCCOCC1CO1</smiles>

e b

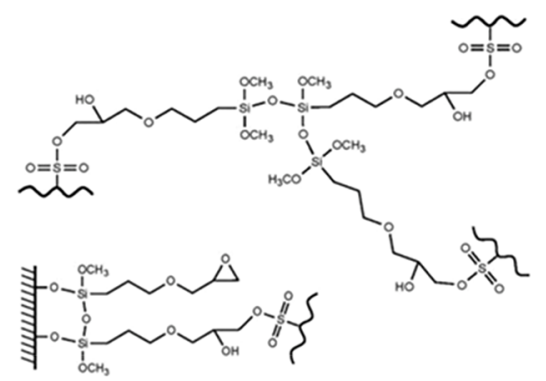

d

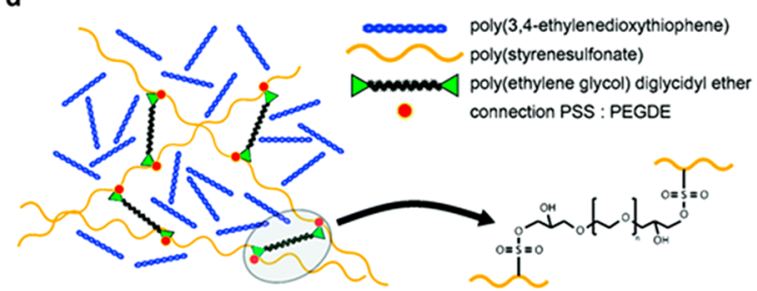

f

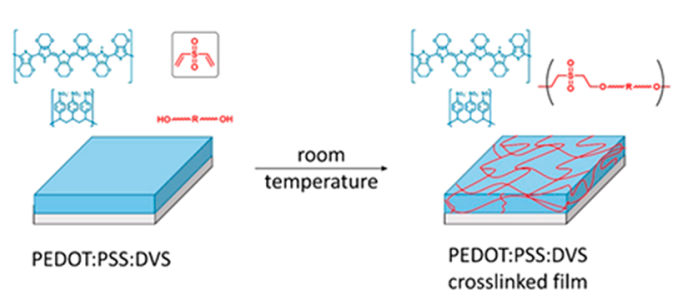

Figure 7. Chemical structure of (a) (3-glycidyloxypropyl)trimethoxysilane (GOPS) and (b) three main chemical bonds formed during cross-linking with GOPS. Adapted with permission from ref 114. Copyright 2016 John Wiley and Sons. (c) Chemical structure of poly(ethylene glycol)diglycidyl ether (PEGDE) and (d) chemical bonds formed between the epoxy rings of PEGDE and $-\mathrm{SO}_{3}{ }^{-}$groups of PSS. Adapted with permission from ref 113. Copyright 2019 The Royal Society of Chemistry. (e) Chemical structure of divinylsulfone (DVS) and (f) schematic showing the cross-linking process of PEDOT:PSS thin films. Reprinted with permission from ref 116. Copyright 2017 American Chemical Society.

between ions and holes gives rise to high capacitance values. Rivnay et al. found that the capacitance scales with the volume of the film as a linear function, revealing a volumetric capacitance of $\mathrm{C}^{*}=39 \mathrm{~F} / \mathrm{cm}^{3} .{ }^{109}$ Proctor et al. looked in more detail at the volumetric capacitance of PEDOT:PSS and proposed a simple model how volumetric capacitance arises. ${ }^{106}$ The model describes capacitance in terms of available "sites" consisting of sulfonate anion/hole pairs, in which ions injected from the electrolyte replace holes (Figure 6c). An injected cation drifts to one of these sites and replaces the hole, creating a double layer capacitor along these planes. Volkov et al. also demonstrated that the major contribution to the capacitance of PEDOT:PSS originates from electrical double layers formed along with the interfaces between PEDOT-rich and PSS-rich interconnected grains. ${ }^{110}$ Processing additives (i.e., ethylene glycol, 4dodecylbenzenesulfonic acid (DBSA), etc.) or annealing of PEDOT:PSS postprocessing are proven successful strategies to enhance the mixed conduction properties of PEDOT:PSS to maximize $C^{*}$ and ion and hole transport properties. ${ }^{77}$ The interaction of hydrated ions and holes within PEDOT:PSS leads to swelling of the material during operation. In fact, PEDOT:PSS films almost double their thickness or volume when immersed in water due to the absorption of electrolyte ions and water through the hydrophilic PSS phase. ${ }^{105}$ Upon dedoping, cation transport takes place in the swollen phase and further expands the volume of the material. In the swelling state, the PSS and PEDOT regions are physically separated from ions and water. ${ }^{111}$ This process is reversed when the electrochemical potential changes, the material shrinks again, and ions and water are expelled back into the electrolyte (Figure 6d).

Swelling and shrinking of PEDOT:PSS occurs repeatedly with every doping/dedoping cycle in aqueous electrolytes, which stresses the material and can lead to deformation and delamination. ${ }^{113}$ In fact, pristine PEDOT:PSS is unsuitable for in vitro bioelectronic applications due to its water solubility. Both thin films and 3D structures made of PEDOT:PSS can be rendered water-stable by using cross-linking agents as additives during processing and thermally activating the cross-linking reactions postprocessing. The most commonly used cross-linker for PEDOT:PSS is the (3-glycidyloxypropyl)trimethoxysilane (GOPS) (Figure 7a). The cross-linking reaction occurs via three main reactions that have been described in detail by Hakansson et al. ${ }^{114}$ (Figure $7 \mathrm{~b}$ ). Strong chemical bonds are formed between GOPS-PSS, GOPS-GOPS, and GOPS-substrate and guarantee stable PEDOT:PSS electrochemical operation within in vitro devices without dissolution or delamination. However, the cross-linking strategy with GOPS comes at the expense of reduced mixed conduction properties of PEDOT:PSS. El Mahmoudy et al. demonstrated that the benefits of stable electrochemical operation of PEDOT:PSS cross-linked with GOPS come at the expense of a decrease in swelling and an increase in the electrochemical impedance. ${ }^{115}$ This indicates that the ionic mobility is also reduced due to the formation of the hydrophobic silane regions within thin films and 3D structures.

Alternative cross-linking strategies involve the use of nonsilane-based cross-linkers, such as poly(ethylene glycol) diglycidyl ether (PEGDE). ${ }^{113}$ (Figure $7 \mathrm{c}$ ). Similar to GOPS, 


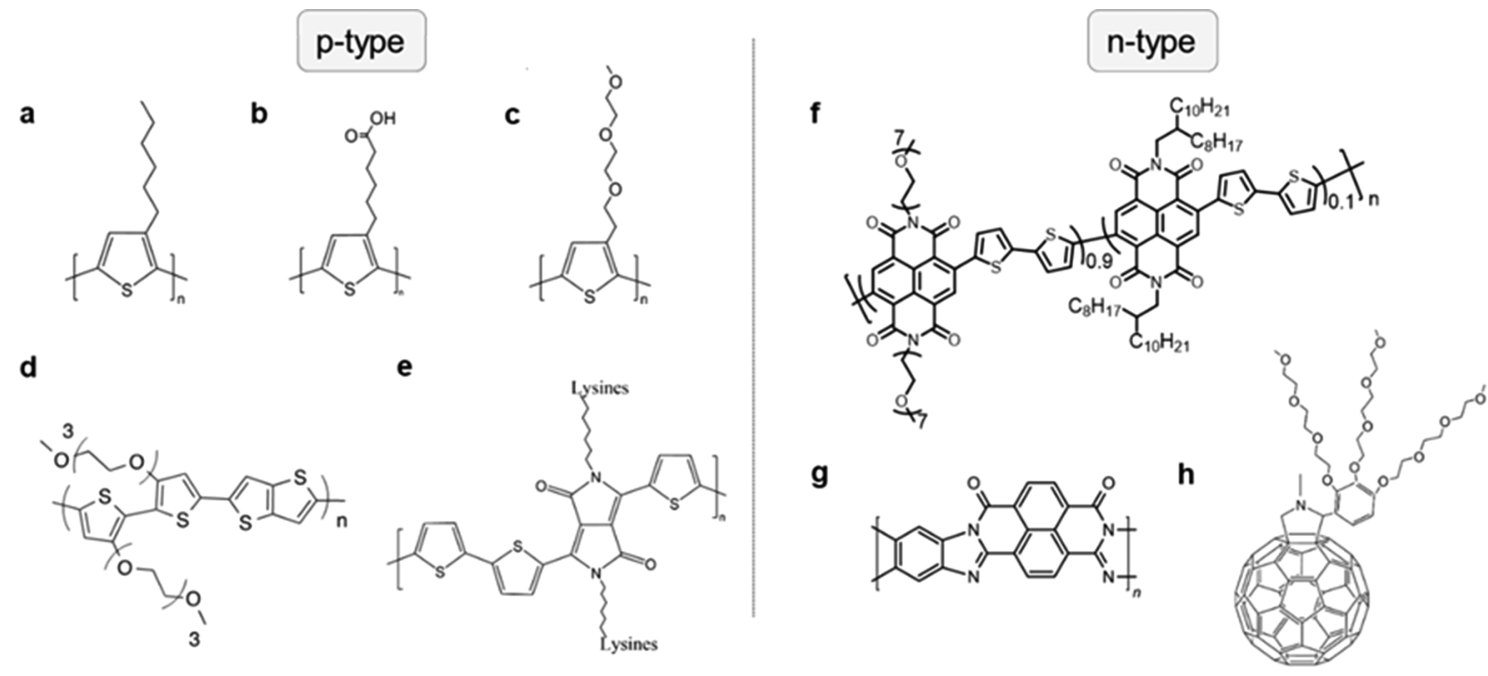

Figure 8. Chemical structures of organic semiconducting materials that have been used for in vitro bioelectronic applications or as building blocks for organic bioelectronics devices. Left panel shows p-type materials, that is, redox active hole conductors, (a) poly(3-hexylthiophene) [P3HT], (b) poly(3-carboxy-pentyl-thiophene) [P3CPT], (c) poly (3-\{[2-(2-methoxyethoxy)ethoxy]methyl\}-thiophene-2,5-diyl) [P3HT-EG], (d) poly (2-(3,3'bis (2-(2-(2-methoxyethoxy)ethoxy)ethoxy)-[2,2'-bithiophen]-5-yl)thieno[3,2-b]thiophene) [p(g2T-TT)], and (e) poly\{2,2'-[(2,5-bis $(2-$-hexyldecyl)-3,6-dioxo-2,3,5,6-tetrahydropyrrolo[3,4-c]pyrrole1,4-diyl)dithiophene]-5,5'-diyl-alt-thiophen-2,5-diyl\} [DPP3T-L]. Right panel shows n-type materials, that is, redox active electron conductors, (f) poly $\left(N, N^{\prime}\right.$-bis (7-glycol)-naphthalene-1,4,5,8-bis(dicarboximide)-co-2,2'-bithiophene-co- $N, N^{\prime}$ bis(2-octyldodecyl)-naphthalene-1,4,5,8-bis(dicarboximide)) [P-90], (g) poly(benzobisimidazobenzophenanthroline) [BBL], and (h) 2-(2,3,4tris(methoxtriglycol)phenyl) [60]fulleropyrrolidine [C60-TEG].

the reaction takes place between the delocalized electrons on the PSS chain and the epoxy ring of PEGDE. In this case, due to the double epoxy ring at both ends of the PEGDE molecule, PSS chains are directly interconnected without the intermediate bonds between silanes resulting in superior conduction properties compared to GOPS cross-linked PEDOT:PSS (Figure 7d). Divinylsulfone (DVS) (Figure 7e) has been also used for cross-linking PEDOT:PSS thin films ${ }^{116}$ and mesoporous scaffolds for 3D cell culture. ${ }^{117}$ Unlike GOPS and PEGDE, DVS reacts with nucleophilic (alcohol and amine) residues in the PEDOT:PSS formulation, creating a network of insoluble chains that render the material stable during operation (Figure $7 \mathrm{f}$ ). ${ }^{116}$ Furthermore, DVS results in reduction of the Young's modulus of the film when compared with pristine PEDOT:PSS, making possible PEDOT:PSS free-standing electrodes. ${ }^{118}$ Finally, cross-linker free approaches for stable PEDOT:PSS thin films have been developed. One interesting study by Kim et al. showed that treatment of PEDOT:PSS films with acid yielded highly conducting devices, likely due to removal of excess PSS. ${ }^{119}$ A later study by the same group demonstrated the use of highly crystalline PEDOT:PSS films to modulate cardiomyocyte beating via direct electrical stimulation. The incorporation of the crystalline PEDOT:PSS into microelectrode arrays showed excellent performance, as well as long-term underwater stability and biocompatibility. Electropolymerized PEDOT:PSS electrodes have also been used to interface with neurons and to build metabolite biosensors. ${ }^{120}$ However, although electropolymerized PEDOT:PSS often yields highly conducting films, the ease of processing of chemically cross-linked PEDOT:PSS combined with its commercial availability have ensured the dominance of chemically prepared PEDOT:PSS.

2.1.4. Undoped Conjugated Polymers. Unlike doped CPs, undoped CPs exhibit notoriously low conductivity in their pristine state. Undoped CPs with excellent mixed conduction properties and stable operation in physiological electrolytes have been recently synthesized via chemical synthesis routes. Many recent reviews discuss the synthesis and the mixed conduction properties of these newly established CPs for bioelectronics. ${ }^{55,121-124}$ These semiconducting polymers do not contain a bulky ionic phase, and therefore, their mixed conduction properties are inherently different compared with PEDOT:PSS. Usually, side chain engineering is applied: hydrophilic side chains are attached on a rigid polymer backbone to promote ionic conduction, while good electronic conduction is maintained. ${ }^{125}$ Among many side chain chemical groups used (e.g., hydroxyl, lysines), ethylene glycol-based side chains are almost exclusively used ${ }^{126,127}$ because of their hydrophilicity and biocompatibility. The general mechanisms of redox activity of these polymers is as follows: upon application of a bias, ions are injected into the material and transported through the hydrophilic network formed by the glycol side chains to compensate for electronic charges that are located in the rigid polymer backbone. ${ }^{128}$ Similar to PEDOT:PSS, these undoped CPs are also ideal volumetric capacitors, with high $C^{*}$ values and good ion and electron (or hole) conduction. ${ }^{83}$ By fine-tuning side chain engineering, electrochemical doping can happen at low voltages (as low as $0.05 \mathrm{~V}$ vs $\mathrm{Ag} / \mathrm{AgCl}$ ). ${ }^{129} \mathrm{The}$ understanding of all these processes is ongoing and many recent reports establish structure-morphology-property relations necessary to understand how these materials operate in aqueous electrolytes. ${ }^{83,130,131}$ Side chain engineering has been applied to synthesize both p-type $\mathrm{e}^{132}$ (hole transport) and n-type $\mathrm{e}^{133}$ (electron transport) CPs. Figure 8 shows the chemical structures of representative CPs that either have been used for in vitro bioelectronics or showed a step change for development of organic bioelectronic devices. Polythiophene backbones functionalized with side chains containing different functional groups are successful examples. P3HT (Figure 8a) is a CP with good optoelectronic properties that has been used extensively for photostimulation of cell activity ${ }^{134}$ and for biosensing. ${ }^{135}$ Furthermore, a number of CPs based on 


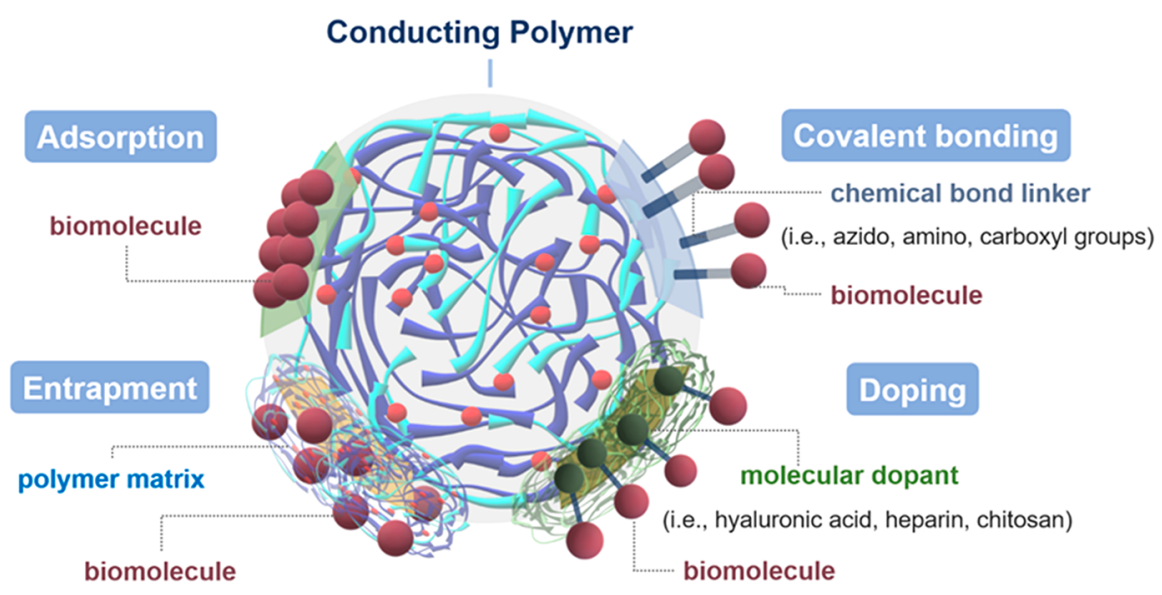

Figure 9. Schematic illustration showing the different biofunctionalization strategies.

polythiophene functionalized with hydrophilic side chains have been explored as the channel material of organic electrochemical transistors (OECTs) (Figure 8b,c). ${ }^{126,136-138}$ In addition, other $\mathrm{CP}$ backbones have been functionalized with glycol side chains and show great potential to be used as bioelectronic interfaces. Glycolated 2-(thiophen-2-yl)thieno[3,2- $b]$ thiophene (2T-TT) polymers, such as poly(2-(3,3'-bis(2-(2-(2-methoxyethoxy)ethoxy) ethoxy)-[2,2' -bithiophen $]-5-y l)$ thieno $[3,2-b]-$ thiophene) $(\mathrm{p}(\mathrm{g} 2 \mathrm{~T}-\mathrm{TT}))^{129}$ (Figure $\left.8 \mathrm{~d}\right)$ and diketopyrrolopyrrole $\left(\mathrm{DPP}^{139}\right)$ (Figure $8 \mathrm{e}$ ) are representative examples, with state-of-the-art mixed conduction properties and stability in aqueous electrolytes. Interestingly, Ohayon et al. showed that lysinated side chains can be attached to a DPP CP backbone to improve adhesion of primary neuron cultures without the need for additional biopolymer coatings. ${ }^{140}$ However, the development of $n$-type CP materials with mixed conduction properties operating in aqueous electrolytes is challenging. The injection of an electron from a metal electrode into an $n$-type CP interfacing an electrolyte, leads to electrochemically reduced chains that are likely to react with molecular $\mathrm{O}_{2}{ }^{141}$ This leads to faradaic side reactions, which are potentially responsible for the limited mixed conduction properties and the electrochemical instability of ntype CPs. ${ }^{142}$ Despite these challenges, recent developments proved that $n$-type materials can be synthesized with operational stability in aqueous solutions and in contact with the biological milieu. ${ }^{141,143}$ Recently, Yang et al. reported the development of a highly conducting n-type polymer based on poly(benzimidazobenzophenanthroline) (BBL) (Figure 8g) doped with poly(ethylenimine), which shows great potential to be used in a number of bioelectronic applications. ${ }^{144}$ Although n-type CPs for in vitro bioelectronics applications are scarce, the recent advancement of n-type CP mixed conductors has enabled new technological opportunities such as complementary logic circuits with high gain, ${ }^{138}$ amplifiers for multiscale real-time ion detection and monitoring, ${ }^{145}$ and biofuel cells for powering in vitro bioelectronic devices. ${ }^{146}$ In addition, these n-type CPs show great potential to be used for metabolite biosensors with simplified design. ${ }^{147,148}$ Finally, it is worth noting that other organic semiconductors (i.e., fullerenes, Figure $8 \mathrm{~h}$ ) have been recently introduced as efficient n-type mixed conductors and show great promise to expand the library of materials for bioelectronics applications. ${ }^{149}$

\subsection{Organic Bioelectronic Interfaces}

Bioelectronic interfaces can be formed with subcellular components, individual cells, cell monolayers, 3D cell cultures, organotypic structures, or real tissue. In bioelectronics, the biotic-abiotic interface is undeniably the most important factor for communication between biological systems and electronic components. Biocompatibility of the abiotic material is crucial for maintaining living cells, the formation of a lasting interface, and the accurate expression of biological mimicry. As described in the previous sections, organic electronic materials can be engineered for the purpose (i.e., via synthetic chemistry) or tailored (i.e., electrically, chemically, or topographically) to support and manipulate cell growth, thus minimizing the mismatch between hard electronics and soft living tissues.

The distinctive features of organic electronic materials were first demonstrated three decades ago by Langer and co-workers in their pioneering work on the ability of PPy to regulate cellsubstrate interactions and subsequently control cell shape and growth. ${ }^{150}$ Specifically, they showed that alterations in the electroactivity of PPy can play a key role in the fate and the function of mammalian cells. Switching from oxidized to reduced (i.e., toward more neutral) state in PPy (bearing fibronectin) resulted in cell rounding and retraction, which in turn can be associated with the detachment or loss of anchoring points between the cells and the substrate-adsorbed fibronectin molecules. Interestingly, despite the dramatic change of cell shape and spreading in the neutral state of the polymer, no loss of cell viability was observed. Since then, several studies on both electrogenic $^{151-154}$ and nonelectrogenic ${ }^{155-157}$ cells confirmed the hypothesis that CPs bearing absorbed biomolecules can be used as functional substrates for controlling crucial functions such as tissue regeneration, as well as for regulating cell adhesion and growth.

In the following sections, we highlight the main strategies that have been used to improve the interface between CPs and biological systems. One strategy involves the incorporation of biomolecules that can promote cell adhesion, growth, or differentiation. Another strategy is the modification of surface properties via the introduction of nano- or microtopographical features at the interface. Finally, an alternative strategy involves the inherent ability of CPs to electrochemically promote cell adhesion. Here, rather than giving an exhaustive review on $\mathrm{CP}$ modification for enhanced biocompatibility, we will showcase key examples, highlighting the features of each method and their potential for in vitro bioelectronic applications.

2.2.1. Surface Functionalization. Surface functionalization of CPs has been a widely explored strategy to improve cell attachment and growth. Typically, this can be achieved by 
incorporating extracellular matrix proteins (i.e., collagen, ${ }^{158,159}$ fibronectin, ${ }^{160,161}$ and laminin ${ }^{162,163}$ ). Alongside improving cell-surface interactions or reducing (bio)fouling, biofunctionalization of CPs has been also used as a strategy to boost their intrinsic properties (i.e., wettability, ${ }^{164,165}$ electroactivity ${ }^{166}$ ). Typically, biological molecules, or other functional groups, can be introduced through (i) adsorption, (ii) entrapment, (iii) covalent bonding, or (iv) doping processes (Figure 9). ${ }^{167,168}$ For the fundamental principles of surface biofunctionalization, the experimental techniques, and the associated approaches, we refer the readers to exhaustive reviews on the subject. ${ }^{169-171}$

2.2.1.1. Adsorption. In physical adsorption, a functional unit (e.g., protein, peptide, nucleic acid) is adsorbed at the polymer surface via electrostatic interactions between charged species and the polymer matrix. In such a case, biomolecules can be attached on the polymer surface through hydrogen bonds, van der Waals forces, or hydrophobic interactions. This method is considered simple and fast, and usually has minimal effect on the electroactivity of the polymer. However, the involved interactions are considered nonspecific, meaning that surrounding biomolecules or proteins may influence the selectivity and disturb the cell/electrode interface. Additionally, the adsorbed biomolecules are susceptible to changes in physiological and environmental conditions (i.e., $\mathrm{pH}$, temperature) and may leach off the surface, rendering the surface inactive. ${ }^{166}$ Adsorption was massively utilized for the development of the first generation of biosensors, ${ }^{172,173}$ as well as in the early studies of CP biointerfaces ${ }^{161,174-177}$

2.2.1.2. Entrapment. In the case of entrapment, a functionalizing agent is mixed with the conducting polymer (or monomer prior to synthesis), and via electrochemical polymerization or other deposition techniques, these functional species are entrapped in the polymeric matrix of the growing film. Adjusting the polymer film properties (i.e., thickness, roughness) in conjunction with the size of the functional unit leads to formation of free active moieties within the bulk and at the surface of the CP matrix. Both adsorption and entrapment can be directly used to modify the polymer surface and do not require additional mediators or chemical modifications. A representative example was demonstrated by Martin et al. with the entrapment of nerve growth factor into both PPy and PEDOT films via electrochemical polymerization. ${ }^{178}$ Validation studies with PC-12 cells cultured on both materials exhibited adequate adhesion and differentiation, as well as highly extended neurites.

2.2.1.3. Covalent Bonding. Covalent chemistry enhances the binding of bioactive molecules and the stability of the resulting CP. This can be done both during CP synthesis and postpolymerization. ${ }^{179,180}$ Various approaches have been suggested for the covalent bonding of biomolecules on the surface of conducting polymers, including the use of selfassembled monolayers (SAMs), ${ }^{181}$ functional groups (i.e., $-\mathrm{NH}_{2}$, sulfur, carboxylic acid), ${ }^{182-185}$ or surface treatment processes (i.e., ion implantation ${ }^{186}$ ). Covalent modification of CPs has been also used as a more controllable approach to incorporate bioactive molecules onto the surface of CPs. ${ }^{179,187-192}$ A number of studies have focused on the covalent attachment of the nerve growth factor (NGF) to improve neurite outgrowth using several strategies including cross-linking, ${ }^{151}$ copolymers bearing ester units for the development of scaffolds, ${ }^{174}$ and amine-ester coupling via the use of poly(lactic-co-glycolic acid) (PLGA) fibers. ${ }^{193}$ Surface modification by peptides has been widely explored as a means of introducing extracellular matrix components, hence improving the biocompatibility and bioactivity of CPs. Examples of such strategies include the incorporation of fibrillar collagen with chondroitin sulfate on 3D PPy structures, ${ }^{159}$ as well as the incorporation (via a terminal cysteine residue) of the most common peptide motif responsible for cell adhesion, Arg-GlyAsp (RGD). ${ }^{184,190}$ CPs based on PEDOT with functional groups have been widely explored. CPs consisting of PEDOT bearing side chain amine groups have shown improved cellular response, specifically cell adhesion and proliferation, ${ }^{157}$ laying the foundation for the design of an electroactive RGD-based PEDOT-peptide $\left(\mathrm{RG}^{\mathrm{E}} \mathrm{D}\right)$ conjugate to promote further both cell attachment and growth. ${ }^{194}$ In a similar study, YIGSR-based peptides were covalently immobilized onto carboxylic acid functionalized PEDOT copolymer using a spacer molecule (PEG). ${ }^{195}$ These films exhibited better cell attachment compared to peptides linked to the surface without a spacer unit (Figure 10a). A facile method for the covalent bonding of

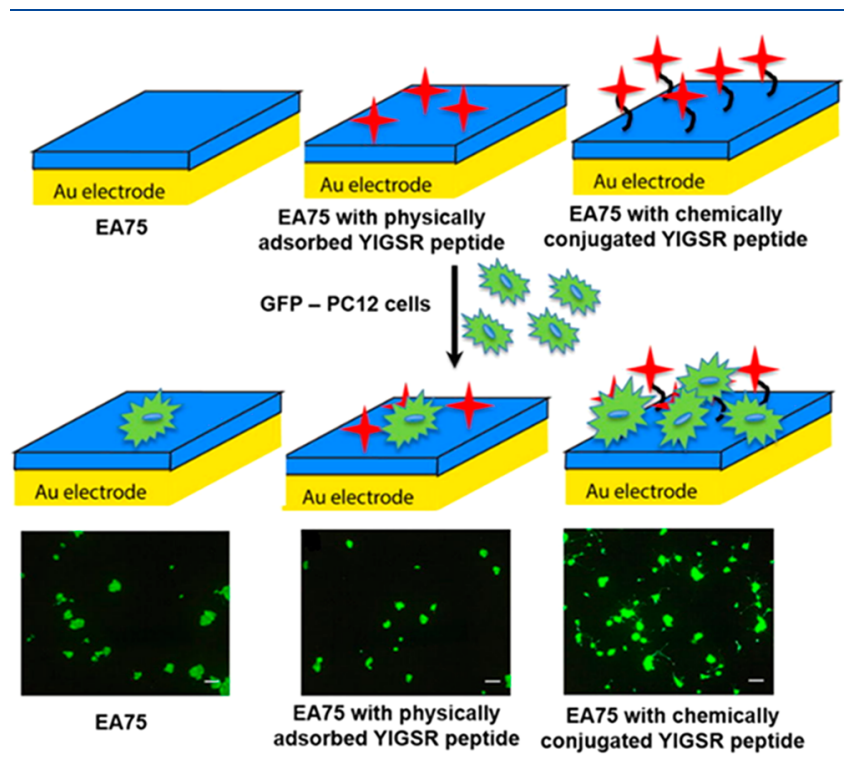

Figure 10. Schematic illustration showing the surface modification route (control, physically adsorbed, chemically conjugated) for carboxylic acid functionalized PEDOT copolymer films with a nonapeptide derived from laminin and the corresponding fluorescence images of PC-12 cells (after $72 \mathrm{~h}$ ) cultured on top of the corresponding PEDOT based films (scale bar is $100 \mu \mathrm{m}$ ). Adapted with permission from ref 195. Copyright 2016 Elsevier.

biomolecules to PEDOT:PSS was also presented by our group by mixing PEDOT:PSS with poly(vinyl alcohol) in order to introduce $-\mathrm{OH}$ groups at the surface. This surface functionality allows for the incorporation of SAMs via condensation reaction, hence providing a universal means to incorporate bioactive molecules such as the extracellular matrix derived polypeptide poly(L-lysine). ${ }^{191}$ A cell repellent surface was recently developed by Zhu et al. based on a phosphorylcholine grafted EDOT, mimicking the cell membrane. ${ }^{154}$ The goal of this study was to inhibit nonspecific binding of proteins, while allowing the selective adhesion of specific cells for long-term stable operation of the devices when interfaced with cells. Moreover, this synthetic route allowed for the covalent coupling with peptide ligands, which can promote specific interactions with target cells.

2.2.1.4. Doping. The doping process is another way to incorporate charged bioactive molecules such as heparin, ${ }^{196}$ hyaluronic acid, ${ }^{196,197}$ chondroitin sulfate, ${ }^{197-199}$ and dextran 
a
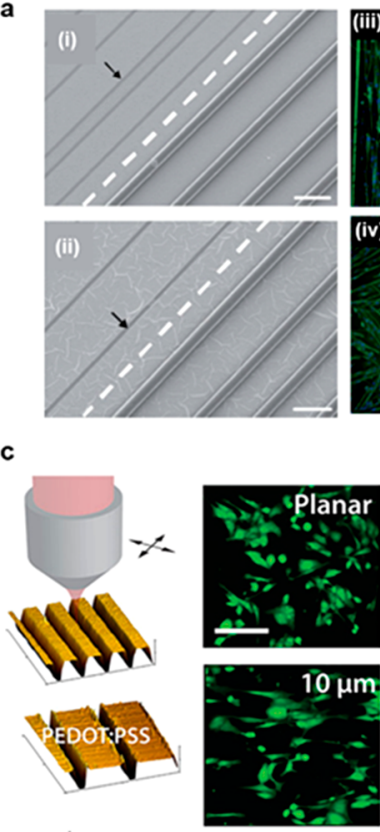
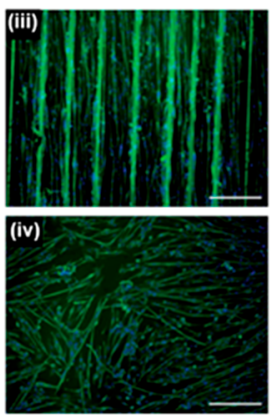

b

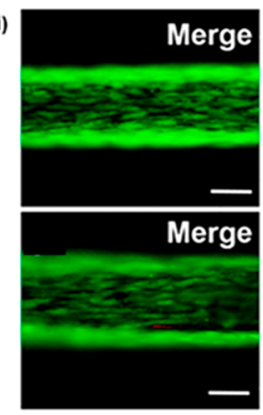

(ii)

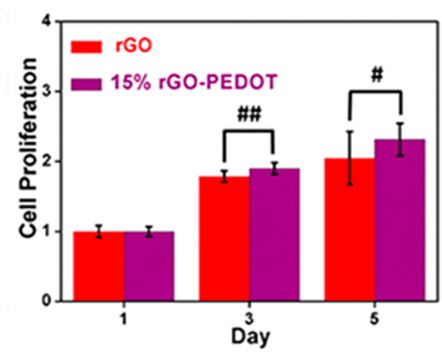

d
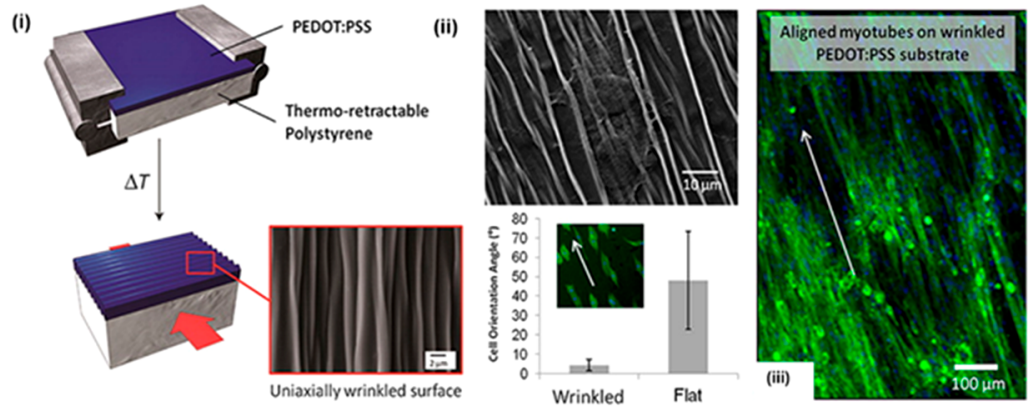

Figure 11. (a) SEM images of aligned PLA:PLGA and PPy fibers doped with (i) pTS and (ii) HA. Fluorescence images showing differentiated muscle cells cultured on PPy/pTS substrate (iii) with and (iv) without the PLA:PLGA fiber array. Adapted with permission from ref 212 . Copyright 2009 WILEY-VCH Verlag GmbH \& Co. KGaA, Weinheim. (b) (i) Live-dead assay of the MSCs growing on rGO microfiber and rGO-PEDOT composite microfiber and (ii) proliferation data of MSCs cultured for 5 days on rGO and the hybrid rGO-PEDOT microfibers. Adapted with permission from ref 213. Copyright 2016 American Chemical Society. (c) Fabrication of 3D micropatterned grooves via femtosecond laser patterning of PEDOT:PSS films and fluorescence images showing cell (HL-1) adhesion in planar and patterned substrates. Adapted with permission from ref 214 . Copyright 2017 American Chemical Society. (d) (i) Schematic showing the development of micro- and nanowrinkles on PEDOT:PSS via heatshrinking process of a thermo-retractable polystyrene substrate. (ii) SEM images of C2C12 (murine skeletal muscle cells) adhered on nanowrinked PEDOT:PSS substrate and comparison of cell adhesion properties between flat and nanowrinkled (visualized in the fluorescence inset image) films. (iii) Fluorescence image of myotubes on the microstructured samples supported by human fibroblast feeder layer. Adapted with permission from ref 215. Copyright 2013 American Chemical Society.

sulfate $^{197,200,201}$ in CPs. Incorporation of chemical dopants can modify the CP properties including surface topography, electrochemical properties, wettability, biocompatibility, and bioactivity, affecting the subsequent adsorption of biomolecules at the surface. ${ }^{198,202}$ Here, drawbacks may arise from the increase in surface roughness and the limited access to the active binding sites at the surface of the polymer. A more recent work by Molino et al. demonstrated the electropolymerization of PEDOT with a range of biological (i.e., dextran sulfate, chondroitin sulfate, alginic acid, and ulvan) and synthetic (i.e., DBSA) dopants to identify the optimal conditions for efficient protein adsorption and enhanced neuronal cell cultures. ${ }^{200}$

2.2.2. Surface Topography. Surface topography plays a key role in the first stages of cell attachment and adhesion and can influence cell fate by regulating cell-cell signaling pathways and cell-microenvironment interactions via mechanotransduction. ${ }^{203-206}$ Topographical cues have been shown to strongly mediate cell morphology and the expression of genes associated with proliferation, migration and differentiation. In the case of bioelectronics, topography is important both for controlling and guiding cellular organization and for minimizing the interfacial cleft between substrate and cells, which in turn improves cellmaterial coupling and hence signal output. ${ }^{207}$ Traditionally, topographical features are introduced via lithography-based approaches, $^{208}$ etching, ${ }^{209}$ self-assembled monolayers, ${ }^{210}$ and laser patterning. ${ }^{211}$ There are three main approaches for the development of topographic surfaces: (i) roughness and (ii) anisotropic and (iii) isotropic patterning. While nanoscale surface roughness is known to affect cell behavior, there is lack of control over nano-irregularities in the case of organic films, thus the efficacy of this approach is rather limited. Hence, focus is placed on patterning methods to create continuous, noncontinuous, and random architectures based on organic electronic materials.

2.2.2.1. Anisotropic Topography. Since the discovery of the "contact guidance" phenomenon, ${ }^{216,217}$ anisotropic topographies have been used to study the cellular morphology and organization on various substrates. This involves the use of oriented $2 \mathrm{D}$ or $3 \mathrm{D}$ structures such as grooves, fibers, or wrinkles that aid cell alignment. Based on this concept Razal et al. demonstrated a hybrid platform consisting of spatially aligned polylactic acid (PLA):PLGA microfibers on electropolymerized PPy substrate, as shown in Figure $11 \mathrm{a}^{212}$ Cultured myoblasts were found to sufficiently adhere and proliferate, adopting a spindle-like shape and elongated morphology, while upon differentiation, they appeared to be well oriented along the fiber axis. In another approach, a hybrid scaffold consisting of reduced graphene oxide ( $\mathrm{rGO}$ ) and PEDOT microfibers was used to culture mesenchymal stems cells (Figure 11b). ${ }^{213}$ Interestingly, the cultures on PEDOT/rGO scaffolds exhibited good cell alignment and viability, as well as improved proliferation and neural differentiation. Similarly, in more recent work, highly aligned fiber structures based on conducting polymers were used to study axonal extension and sprouting from dorsal root ganglia neurons. $^{218}$ 
Controllable and systematic 3D nano- or microstructuring for organic biointerfaces can be also achieved by laser patterning techniques, such as laser ablation and writing. ${ }^{21,220}$ In the former, material is removed under the action of a focused pulsed laser beam. The amount of the removed material and the resolution of the resulting structures depend on the laser wavelength, intensity, and pulse length, as well as on the material properties. Laser writing, on the other hand, enables the formation of $3 \mathrm{D}$ architectures via surface modification or material addition. This technique allows for processing of various materials with a sub-micrometer level resolution. Both approaches have shown great potential for cell guiding and controlled adhesion. Santoro et al. have demonstrated the use of femtosecond laser ablation for 3D patterning of PEDOT:PSS electrodes, as shown in Figure 11c. ${ }^{214}$ The resulting grooved microtopography enhanced cell adhesion, hence decreasing the interfacial cleft resistance without showing any apparent degradation in the quality and the electrical properties of the $\mathrm{CP}$. Cardiomyocytes grown on grooves with the smaller pitch size $(10-20 \mu \mathrm{m})$ were found to spread and adhere better compared to larger $(40 \mu \mathrm{m})$ grooves. This is consistent with previous studies showing that cells preferentially adhere on smaller topographical features, ideally on sub-micrometer scale. ${ }^{221,222}$ Laser writing was recently employed to control the positioning of neurospheres on top of PEDOT:PSS-based MEAs. Given the poor cell adhesion properties of PEGDA, ribbon-like structures with a width of $\sim 12 \mu \mathrm{m}$ and an interspacing of $40 \mu \mathrm{m}$ were positioned between the electrodes in order to confine neurospheres on top of the electrodes and provide guidance for the neurite outgrowth. This resulted in enhanced electrophysiological recording yield. ${ }^{223}$ In another study by our group, we introduced a surface modification and cell patterning route based on laser ablation. In particular, by patterning cell-repelling (perfluorodecyltrichlorosilane, FDTS) SAMs on top of PEDOT:PSS films, we were able to spatially confine cells in laser-ablated regions of various shapes and sizes, with remarkable precision. ${ }^{224}$ Greco et al. demonstrated a novel method for the preparation of micro- or nanowrinkles on $\mathrm{CP}$ surfaces. ${ }^{215}$ As shown in Figure 11d, wrinkle-like structures were produced via a heat-shrinking process with a thermo-retractable polystyrene substrate. In such cases, the mechanical mismatch between the substrate and the top spin-cast PEDOT:PSS layer aided the formation of anisotropically uniaxial micro- and nanowrinkles with various sizes (between 1.45 and $2.45 \mu \mathrm{m}$ ), depending on the PEDOT:PSS thickness. Cells tended to adhere and align on low and narrow ridges rather than on higher and wider ones, exhibiting improved differentiation capabilities as proven by the generation of elongated and multinucleated myotubes.

2.2.2.2. Isotropic Topography. The term isotropic topography refers to surfaces where topographical features are independent of direction and their physical properties are the same at any point or direction. Typical examples include pits, pillars, islands, columns and random fibers. Generally, interfacing cells with isotropic surfaces does not induce cell guidance and elongation while the associated cell response is often cell type-specific. Isotropic patterns have been used for in vitro organic bioelectronics, mainly for interfacing electrogenic cells. Recent work by Tomaskovic-Crook et al. involved the fabrication of PEDOT:PSS micropillars on top of MEAs for the electrical stimulation of human neural stem cells, as shown in Figure $12 \mathrm{a} .{ }^{225}$ As expected, the resulting devices demonstrated enhanced electrical performance versus flat films, as well as a
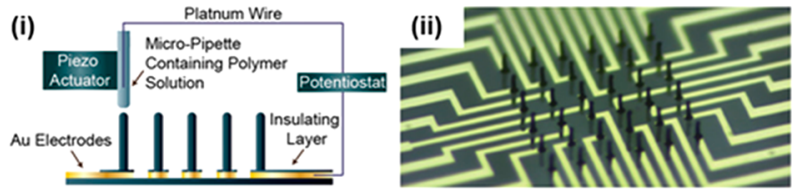

b
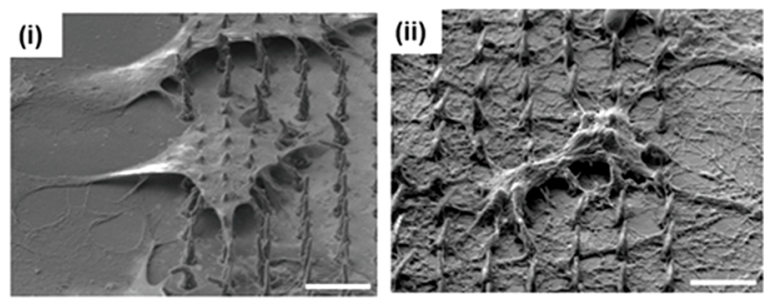

C
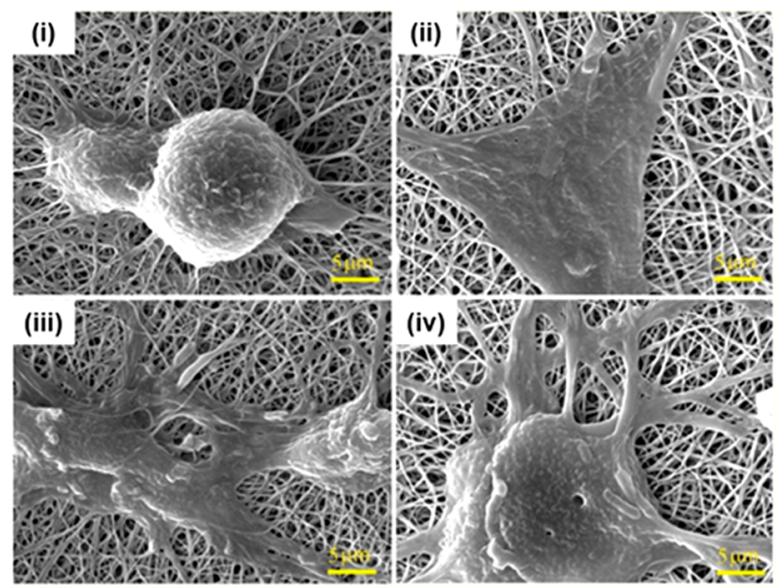

Figure 12. (a) (i) Schematic showing the fabrication of $3 \mathrm{D}$ PEDOT:PSS micropillars using direct write printing technique. (ii) Optical micrograph showing PEDOT:PSS micropillars on top of a photolithographically patterned MEA. Adapted with permission from 225. Copyright 2019 WILEY-VCH Verlag GmbH \& Co. KGaA, Weinheim. (b) SEM images showing (i) HEK-293 cells and (ii) primary cortical neurons cultured on top of rr-P3HT micropillars. Adapted with permission from ref 226. Copyright 2019 American Chemical Society (c) SEM images of mesenchymal stem cell cultures on PEDOT:PSS/PVA/chitosan scaffolds with different PEDOT:PSS wt $\%$ content $(0,0.3,0.6,1)$ : (i) CS/PVA, (ii) CS/PVA/PEDOT:PSS (0.3), (iii) CS/PVA/PEDOT:PSS (0.6), (iv) CS/PVA/PEDOT:PSS (1). Adapted with permission from ref 227. Copyright 2019 Elsevier.

sufficient response under electrical stimulation by means of neural network functionality and differentiation. In a similar manner, Tullii et al. showed the fabrication of high aspect ratio micropillars based on P3HT. ${ }^{226}$ The resulting substrates exhibited high biocompatibility when cultured with human embryonic kidney cells or primary cortical neurons. As shown in Figure $12 \mathrm{~b}$, both cell types exhibited an elongated morphology with the tendency to spread in the $3 \mathrm{D}$ space. Additionally, the close contact between the cells and the micropillars renders the proposed platform optimal for electrophysiological recordings. The use of polymeric fibers to obtain conducting isotropic structures is another commonly used approach for introducing nanotopography, 3D topologies, and high surface to volume ratio. Even though randomly oriented fiber meshes do not provide extensive guidance to cells, they typically exhibit a highly porous morphology (at different scales) and can host high cell density cultures, which is important for modulating the cell response in some applications. On the basis of this, several groups studied the electrical and topographic properties of 

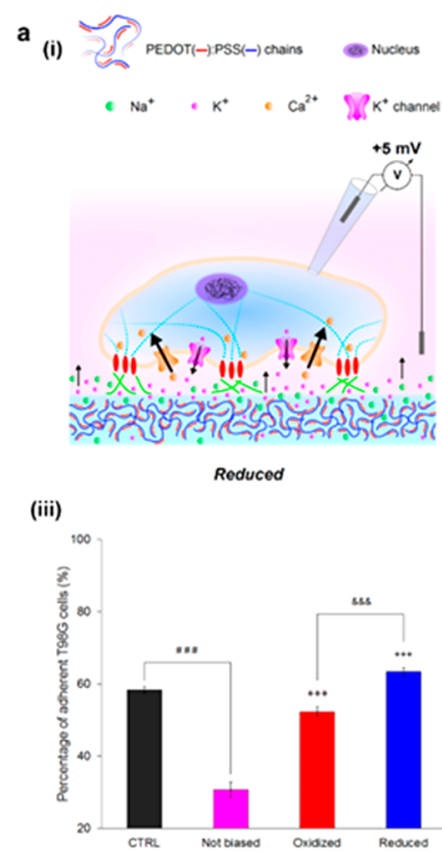

(ii)

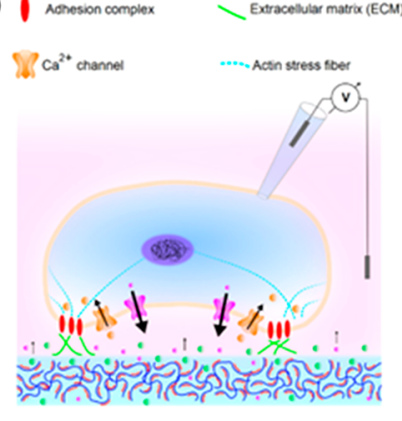

(iv)

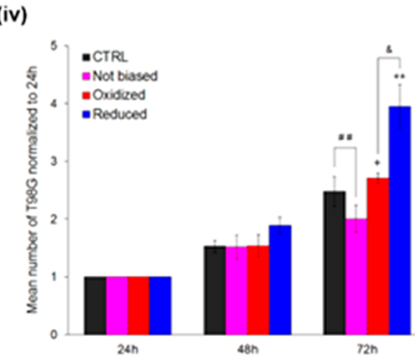

b

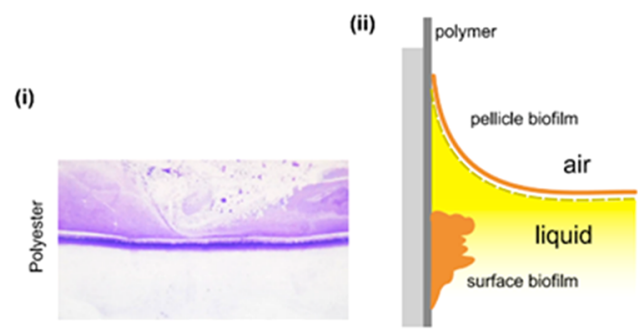

(iii)

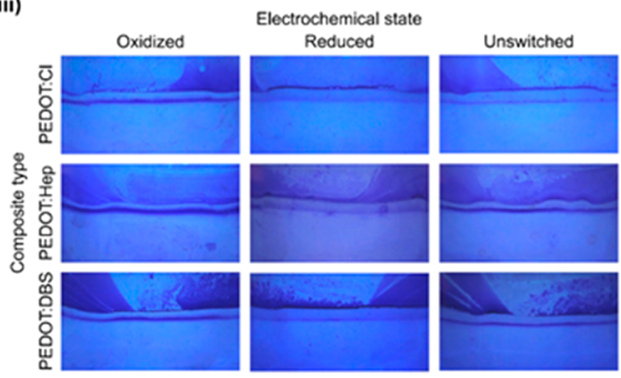

Figure 13. (a) Schematic illustration showing the nanosponge effect on electrochemical cellular stimulation. PEDOT:PSS substrates were used for cells adhesion. The ionic concentration is altered at the film-liquid interface by redox switching: (i) Cations are released from PEDOT:PSS in reduced state, and thus their extracellular concentration increased. This causes a depolarization $(+5 \mathrm{mV})$ of the plasma membrane, which lowers the outward $\mathrm{K}^{+}$ efflux and enhances the inward flow of $\mathrm{Ca}^{2+}$, which in turn enhances cell adhesion. (ii) In oxidized state, there is a higher flow of $\mathrm{K}^{+}$resulting in more polarized membrane potential $(-10 \mathrm{mV})$ and lower $\mathrm{Ca}^{2+}$ flow, which limits cells adhesion and proliferation. Effects of PEDOT:PSS redox state on the T98G cell (iii) adhesion and (iv) proliferation for different time points (24, 48, $72 \mathrm{~h}$ ). Adapted with permission from ref 229 . Copyright 2017 American Chemical Society. (b) Salmonella biofilm formed on redox active PEDOT composites. (i) Photograph of a crystal violet-stained polyester surface showing the presence of the biofilm formed after $24 \mathrm{~h}$ on the PEDOT surface. (ii) Schematic showing the experimental structure of the device. (iii) Optical micrographs showing the formation of biofilm under different redox states of the various PEDOT composites. Adapted with permission from ref 230. Copyright 2017 Springer Nature under Creative Commons Attribution 4.0 International (CC BY 4.0) License, https://creativecommons.org/ licenses/by/4.0/.

conducting fibrous meshes, as well as their potential use in electrical stimulation and in vivo implementation. A representative piece of work from the Schmidt team demonstrated the use of PPy-coated electrospun PLGA nanofibers and the effects of random and oriented fibers on neuronal cell cultures. ${ }^{228}$ Specifically, the resulting electroactive scaffolds were found to maintain the characteristics of conventional electrospun scaffolds (i.e., nanotopography, high surface-to-volume ratio, interconnecting pores) while remaining conducting. Stimulation studies on such neuronal cell cultured scaffolds revealed a higher percentage of neurite-bearing cells, as well as longer neurites in the case of aligned PPy-PLGA fibers. Xie et al. showed a similar concept with conductive core-sheath nanofibers based on poly ( $\varepsilon$-caprolactone) (PCL)-PPy or PLA-PPy, ${ }^{170}$ and more recently Pinna et al. developed electrospun fibers made of PCLPANI using a novel solvent system. ${ }^{640}$ Natural polymers (e,g., chitosan, silk) blended with conducting polymers have been also used for the fabrication of conducting fibrous structures. In a recent study by Abedi et al., PEDOT:PSS and poly(vinyl alcohol) (PVA)/chitosan were blended in various ratios as shown in Figure 12c. ${ }^{227}$ The structures with the highest PEDOT:PSS content resulted in a decrease in the fiber diameter and improved cell adhesion and viability. Fibrous structures, both aligned and randomly oriented, are of particular interest for $3 \mathrm{D}$ cellular structures and will be discussed in more detail in section 3.3 in the context of conducting scaffolds.

2.2.3. Tailoring Biointerfaces via Redox Switching. Changing the oxidation state of conjugated polymers is a proven, versatile way to alter the properties of biointerfaces and take control over cell morphology and critical biological functions such as adhesion, proliferation, and differentiation. ${ }^{150}$ The fundamental mechanisms that regulate such responses are now well understood and can be linked with morphological, physicochemical, and conductivity alterations upon redox switching. Typically, upon oxidation or reduction of conjugated polymers, ions migrate in and out of the polymer compensating the change in the electronic charge on the polymer backbone. The switchable properties of CPs have been widely exploited for regulating ECM protein conformation on top of CP substrates, which in turn can control cell-surface interactions and promote or inhibit cell adhesion and growth. PPy was extensively used in the early studies of CP redox switching effects on biointerfaces, while PEDOT dominated the field later and became the reference material for such bioelectronic applications. Over the years, several variants of those materials have been demonstrated, mainly as a result of chemical modification or dopant incorporation. In a work by Saltó et al., substrates based on PEDOT:Tosylate (Tos) were used to study the attachment of neural stem cells (NSCs) and correlate it with the adsorption of human serum albumin (HSA) in the oxidized or reduced state. The oxidized surfaces exhibited a substantial improvement in cell adhesion compared to reduced surfaces, which was attributed to differences in protein conformation. ${ }^{231}$ Likewise, redox switching was used to investigate possible effects on the adhesion and proliferation of epithelial cells. Cell cultures on PEDOT:Tos electrodes exhibited enhanced proliferation in the case of reduced surfaces in contrast to the oxidized surfaces, attributed to alterations in the conformation of the surface- 

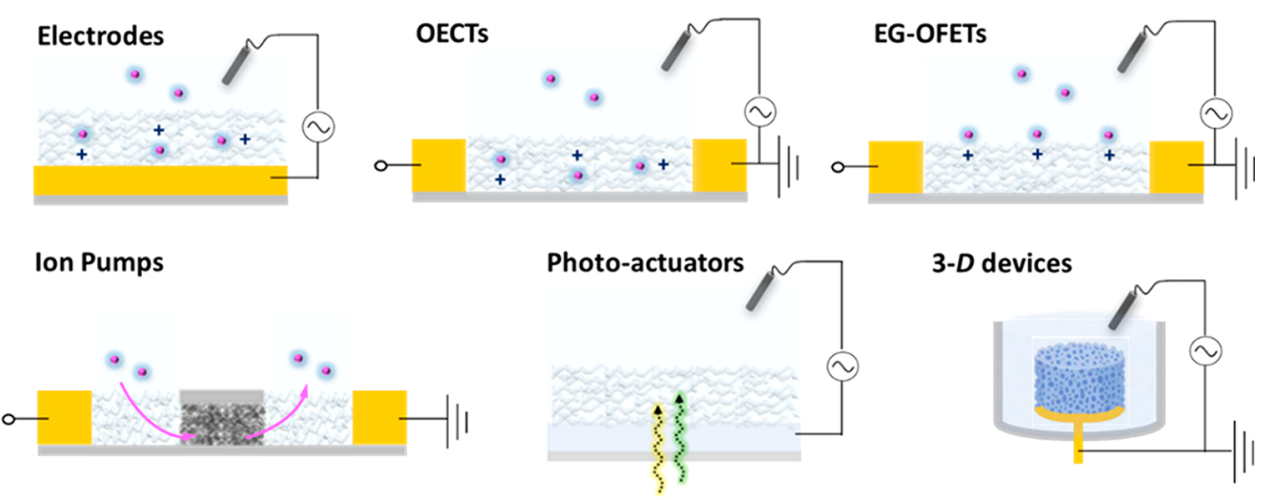

3- $D$ devices

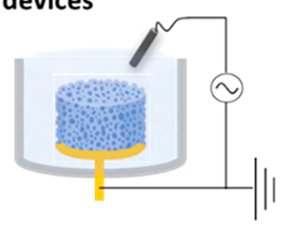

Conjugated Polymer

Substrate/insulator

lon exchangemembrane

- Hydratedlons

Metal contacts

Supporting electrodes

Figure 14. Schematic illustrations of CP-based devices used for in vitro studies.

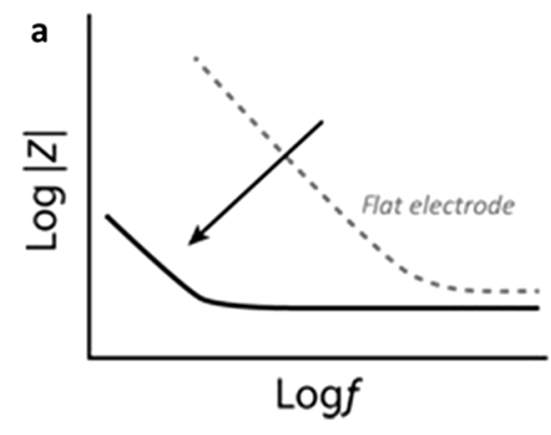

C

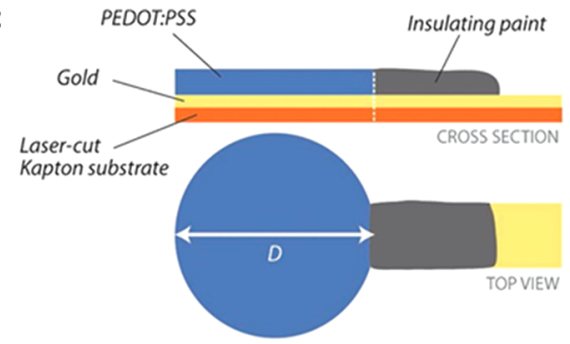

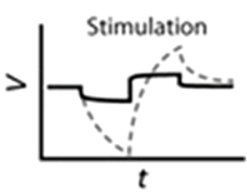

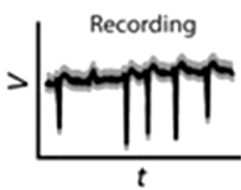

$t$ b

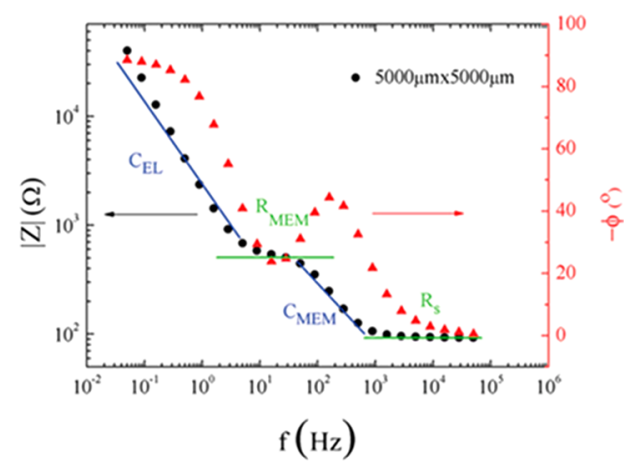

d

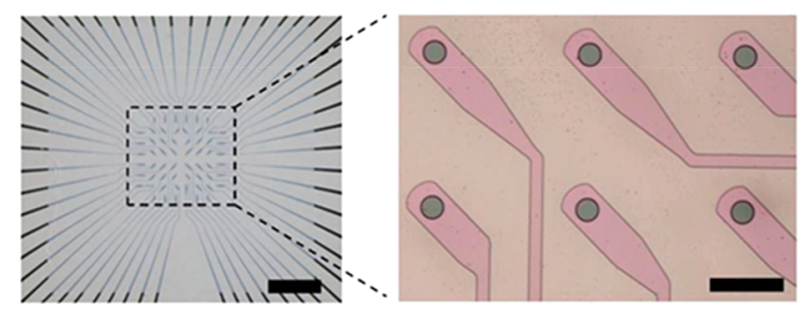

Figure 15. CP-based electrodes: typical measurement output and architectures. (a) Typical response of the impedance magnitude as a function of frequency, the stimulation profile upon a biphasic current pulse, and the signal-to-noise ratio for a flat metal electrode (gray dashed lines) and for a CPcoated metal electrode (balck solid lines). Adapted with permission from ref 87 . Copyright 2017 The Authors, published by AAAS under the terms of the Creative Commons Attribution license, https://creativecommons.org/licenses/by/4.0/. (b) Typical response of the impedance and phase spectra recorded when a barrier tissue is formed directly on the surface of a CP-coated electrode. Adapted with permission from ref 252. Copyright 2019 The Authors. Published by WILEY-VCH Verlag GmbH \& Co. KGaA, Weinheim. (c) Large area PEDOT:PSS coated kapton flexible electrode. Adapted with permission from ref 84. Copyright 2013 WILEY-VCH Verlag GmbH \& Co. KGaA, Weinheim. (d) Transparent microelectrode arrays made with PEDOT:PSS to allow simultaneous optical and electrical measurements of primary cortical cell cultures. Scale bars: left, $1 \mathrm{~cm}$; right, $100 \mu \mathrm{m}$. Adapted with permission from ref 260. Copyright 2021 The Authors. Advanced Science published by Wiley-VCH GmbH under the terms of the Creative Commons CC BY license, https://creativecommons.org/licenses/.

adsorbed fibronectin. ${ }^{188}$ A systematic work by Lundin et al. later showed the effects of various counterions for PPy, such as dodecylbenzenesulfonate, tosylate, perchlorate, and chloride, on the biocompatibility of NSC cultures. It was found that doping PPy with smaller dopants may lead to a loss of biocompatibility, due to the gradual leaching of dopants under physiological conditions. $^{232}$ Herland et al. demonstrated the use of PEDOT with heparin as counterion for immobilizing fibroblast growth factor-2 (FGF2), resulting in an electrochemical bioswitch. In the neutral state, the surface promotes the presence of FGF2 supporting proliferation of NSCs but inhibiting their differ- entiation. Electrochemical oxidation, on the other hand, decreases the bioavailability of FGF2, resulting in a decreased proliferation but increased differentiation into neural cell types. $^{233}$

In another study, PEDOT:Tos was used to enable control of fibronectin conformation over macroscopic areas and assessed via Förster resonance energy transfer (FRET) imaging. ${ }^{234}$ In an effort to analyze quantitatively and at a molecular level the interactions between single cells and the CP surface, Zhang et al. utilized single cell force spectroscopy combined with electrochemical AFM. It was found that the electrochemical switching 
alters the properties of the molecular bonds as well as the force, the stiffness, and the wettability of the surface. ${ }^{235}$ Another study showed that the effects of electrically switchable surfaces on cell growth can be dependent on the cell type. ${ }^{236}$

More recently, a comprehensive characterization approach comprised of electrophysiology, X-ray spectroscopy, and AFM was used to monitor redox switching effects on thickness (or volume) of PEDOT:PSS films. Interestingly, the authors were able to observe a change in the volume of the film and the concentration of ions, indicative of sponge-like behavior. This effect was found to modulate the electrochemical equilibrium at the film-liquid interface, which in turn can dynamically influence cell adhesion and proliferation. ${ }^{229}$ The electrochemical tunability of CPs has also been tested for microbiology applications. A representative study by Carretero et al. demonstrated the use of PEDOT composites to regulate bacterial (Salmonella typhimurium) metabolism via redox state alterations $^{230}$ (Figure 13b). Bacteria/CP interfaces and interactions will be covered in section 3.2.3.

The above-mentioned studies further highlight the superior processability and chemical tunability of conjugated polymers. The incorporation of novel techniques has opened new directions in fine-tuning the surface topography and properties of CPs fit for purpose, substantially improving cell-chip coupling.

\subsection{Organic Bioelectronic Devices}

A wide range of bioelectronic devices are used to monitor or stimulate cellular activity. The electroactive area of these devices is made either solely from CPs or from CP-based material composites, in direct contact with the cells. These materials can be micropatterned using lithography ${ }^{237}$ and solution processing techniques on both glass and flexible substrates. ${ }^{238}$ Figure 14 illustrates the commonly used device types, including electrodes, transistors, ion pumps, photoactuators, and nonplanar/3D devices. This section will describe in detail how each of these devices operate and are used for translating biological events in vitro into electrical signals. Three-dimensional devices are described in section 3.3.

2.3.1. Electrodes. A CP coated on a metal electrode lowers the impedance per unit area by several orders of magnitude, ${ }^{84}$ due to the high volumetric capacitance of CPs ${ }^{239,240}$ (Figure 15a). This property can significantly decrease the footprint of the electrodes without sacrificing their sensitivity, that is, the signal-to-noise ratio (SNR). ${ }^{241}$ The stimulation profiles of such electrodes are also improved for a given biphasic current pulse, and the recording quality is enhanced making the electrodes sensitive to smaller ionic signals arising from cellular activity. ${ }^{242}$ In addition, the soft and dynamic nature of CP coatings can improve the biotic/abiotic interfacing for both short-term and long-term operation. ${ }^{243}$

Owing to a simpler device design compared to three-terminal devices, electrodes can be microfabricated in dense arrays (i.e., microelectrode arrays, MEAs), typically used for in vitro recordings and stimulation of electrogenic cells. ${ }^{244,245} \mathrm{PE}-$ DOT:PSS-based MEAs have been successfully used to monitor neuronal activity with improved SNR, ${ }^{246}$ owing to the intimate bioelectrode interface and inherent low impedance. ${ }^{247}$ Alternative ways to improve coupling include nanostructuring, as well as the addition of 3D structures. ${ }^{248,249}$ These CP-based electrodes are used for electrochemical measurements such as electrochemical impedance spectroscopy (EIS), chronoamperometry (CA), and cyclic voltammetry (CV) to extract the parameters relevant for the in vitro biological system being studied. Apart from neuronal recordings and stimulation, CPcoated electrodes are used to monitor the formation of barrier tissue from nonelectrogenic cells in vitro ${ }^{250}$ (see section 3.2). The formation of a biolayer on the surface of CP-coated electrodes increases the impedance in the midfrequency range (Figure 15b). ${ }^{251,252}$ Similar impedance responses are observed when PEDOT:PSS electrodes are interfaced with cell membranes, (see section 3.1). ${ }^{253}$

Advances in microfabrication enable the realization of electrodes with customized features to accommodate specific biological needs (Figure 15c). ${ }^{254,255}$ For example, Susloparova et al. showed that low impedance, highly transparent MEAs made of PEDOT:PSS alone, without the use of gold pads, could be used to probe the activity of neurons in vitro. ${ }^{256}$ The transparency of PEDOT:PSS was utilized by Middya et al. to fabricate MEAs that are able to monitor cell activity with both optical imaging and electrical measurements ${ }^{257}$ (Figure 15d). Finally, solution processed CP coatings allow the employment of printing techniques like inkjet ${ }^{258}$ and screen printing, ${ }^{259}$ which significantly simplifies fabrication of electrodes and large area MEAs. It is worth noting that PEDOT:PSS coated gold electrodes are already commercially available in a variety of form factors.

2.3.2. Transistors. All transistor technologies used for organic bioelectronics are considered a part of the organic thinfilm transistor (OTFT) family. ${ }^{261}$ The channel is made of organic semiconductors (often CPs) patterned between source and drain contacts and in direct contact with a dielectric/gate electrode couple. ${ }^{262}$ Upon application of a drain-source voltage $\left(V_{\mathrm{DS}}\right)$, electronic current flows through the CP in the channel. A voltage that is applied at the gate $\left(V_{\mathrm{GS}}\right)$ drives charges to the gate/dielectric and channel/dielectric interfaces and controls the current flow. Transistors used as organic bioelectronic interfaces for in vitro studies are required to operate in liquid, often in physiologically relevant electrolytes and in direct contact with tissue. The electrolyte, owing to its high ionic conductance, functions as the dielectric used in solid state OTFTs connecting the external gate. Consequently, an application of a gate bias drives electrolyte ions toward the $\mathrm{CP}$ channel and modulates the current flow. Cells can be cultured directly on the transistor surface and ionic biological signals are recorded as electronic signal changes due to the modulation of the current flowing in the transistor channel. ${ }^{263}$

Electrolyte gated OTFTs can be broadly categorized as organic electrochemical transistors (OECTs) ${ }^{264}$ and electrolyte gated organic field effect transistors (EGOFETs). ${ }^{265}$ OECTs are made from CPs that support mixed ionic and electronic conduction (see section 2.1, materials) allowing ions to penetrate into the bulk of the film and interact with electronic charges within the whole volume of the OECT channel. ${ }^{264}$ In contrast, EGOFETs are usually made of "rigid", highly crystalline CPs or small molecules that are ion impermeable limiting the ion-electron interactions to the (2D) electrolyte/ channel interface (Figure 14). ${ }^{266}$ The following section describes in detail the principles of operation of OECTs and EGOFETs and the different device architectures used for in vitro bioelectronic applications. Other transistor devices have been recently realized and show promise to expand the capabilities of transistor interfaces used for in vitro bioelectronics. Duong et al. demonstrated the liquid-liquid phase separated (LiPS) device allowing for fabrication of OECTs gated with an organic solvent-aqueous electrolyte phase separated liquid. The LiPS 

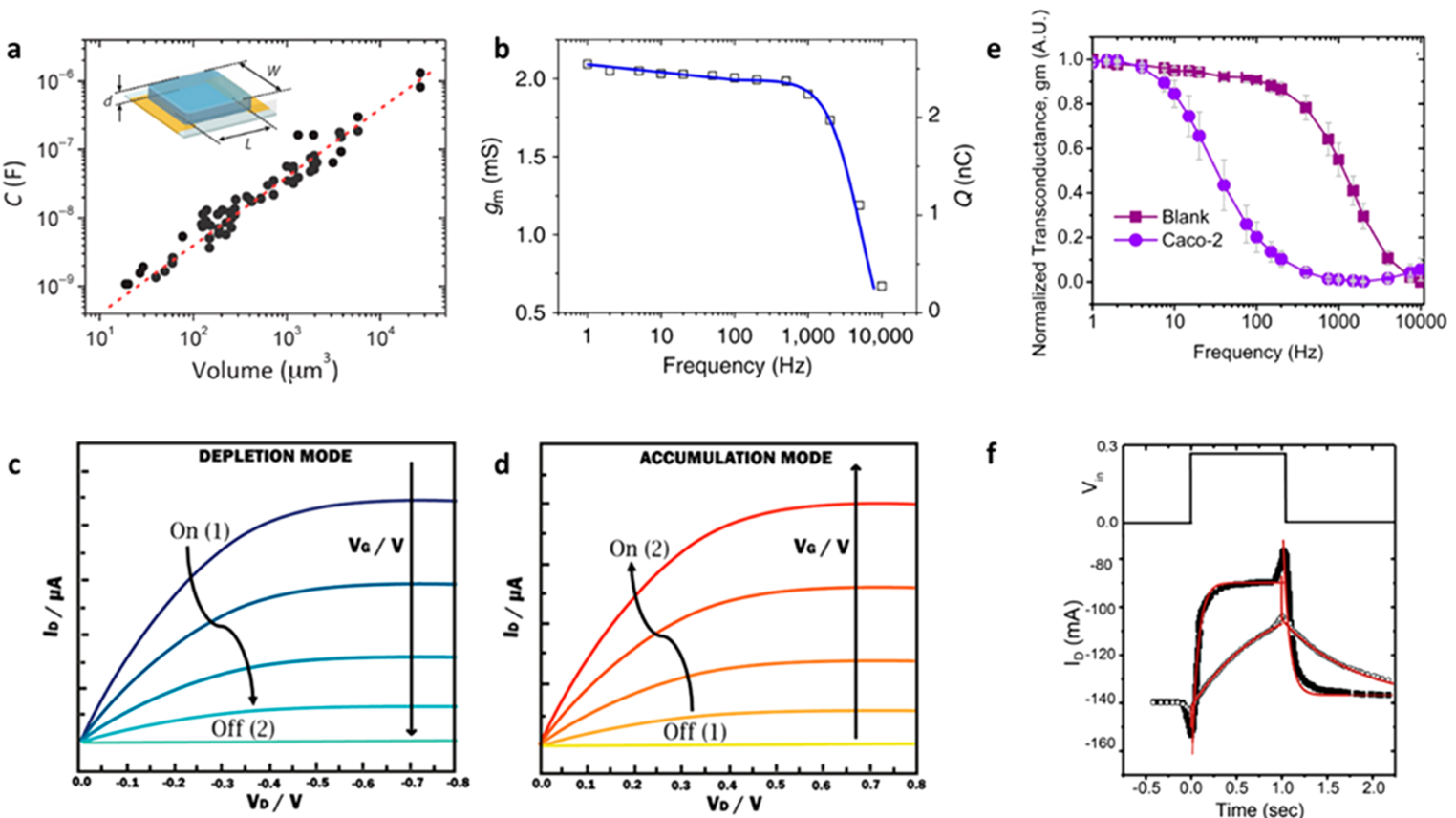

Figure 16. (a) Scaling of capacitance of PEDOT:PSS-based OECT channels with volume $(W \times L \times d)$. Reproduced with permission from ref 109 . Copyright 2015 The Authors, published by AAAS under Creative Commons Attribution-NonCommercial 4.0 International (CC BY-NC 4.0), https://creativecommons.org/licenses/by-nc/4.0/. (b) Typical transconductance vs frequency graph (bandwidth) of PEDOT:PSS-based OECTs. This graph is used to extract the cut-off frequency of the device. Reprinted with permission from ref 272 . Copyright 2013 The Authors, published by Springer Nature under a Creative Commons Attribution-NonCommercial-NoDerivs 3.0 Unported License http://creativecommons.org/licenses/bync-nd/3.0/. Output curves of OECTs made of (c) PEDOT:PSS operating in depletion mode and (d) semiconducting polymers (n- or p-type) operating in enhancement mode (or accumulation). For both modes of operation, the output curves are the steady-state transconductance of the device. Adapted with permission from ref 282. Copyright 2020 The Authors, published by MDPI under Creative Common CC BY license, https:// creativecommons.org/licenses/by/4.0/. (e) Bandwidth of OECTs with (square symbols) and without (circles) cells. The cut-off frequency is significantly reduced when a cell layer is formed on the channel surface. Adapted with permission from ref 283. Copyright 2018 Elsevier B.V. (f) Response time measurements of the OECTs before and after the formation of the biolayers that tightly seal the surface of the channel. Adapted with permission from ref 277. Copyright 2017 Elsevier B.V.

architecture expands the library for OECT-compatible operation to hundreds of water-insoluble materials that have been developed for traditional thin film electronics. ${ }^{267}$ Another novel design demonstrated by Spyropoulos et al. is the internal-ion gated transistor, which uses contained mobile ions within the conducting polymer channel to permit ultrafast response time and allow the monitoring of fast biological events of living organisms. $^{268}$

2.3.2.1. Organic Electrochemical Transistors. A number of recent comprehensive reviews cover in detail several aspects of OECT technology such as device physics, ${ }^{269} \mathrm{CP}$-based channel materials, ${ }^{123}$ and general overview and advances. ${ }^{264,270}$ OECTs can not only transduce ionic biological activity into detectable electronic signals, but in some cases amplify it at source. The volumetric interaction of ionic and electronic charges within the CP channel is the origin of the high transconductance $\left(g_{\mathrm{m}}\right)$, and hence amplification, of OECTs when operated at low $V_{\mathrm{DS}}$ and $V_{\mathrm{GS}}$, typically between 0 and $0.6 \mathrm{~V} \cdot{ }^{271,272}$ The value of $g_{\mathrm{m}}$ is dependent on the thickness and the overall volume of the channel, ${ }^{109}$ scaling linearly with the CP thickness and the channel volume (Figure 16 a). ${ }^{272}$ A typical bandwidth OECT profile (i.e., transconductance vs frequency over a wide range of frequencies) with a cutoff frequency value in the kilohertz range is shown in Figure 16b.

2.3.2.1.1. OECT Types. PEDOT:PSS is the dominant OECT channel material used to date. As PEDOT:PSS is doped, the resulting OECT devices operate in the depletion mode (i.e., application of a gate bias dedopes the channels) (Figure 16c).
Enhancement mode (or accumulation mode) OECTs can be also realized when undoped CPs are used as the channel material (Figure 16d). In that case, the device is initially in the OFF state and application of a gate bias dopes the CP. Albeit less established, enhancement mode OECTs show great potential to expand the implementation of these devices in circuits for bioelectronics. ${ }^{138}$ In addition, as recently shown by Venkatraman et al., ${ }^{271}$ enhancement mode OECTs can be successfully operated in the subthreshold region of operation exhibiting high voltage gain and low power consumption. The OFF-to-ON transition can thus be very appealing for biosensing applications, for example, where the binding event turns the device $\mathrm{ON}$. One prime example of undoped CPs used in enhancement mode OECTs is the p-type material $\mathrm{p}(\mathrm{g} 2 \mathrm{~T}-\mathrm{TT})^{129}$ (see section 2.1 ) that exhibits high $g_{\mathrm{m}}$ and has been used for in vitro studies. ${ }^{273} \mathrm{n}$ Type enhancement mode OECTs have also been developed and used as OECT-based biosensors ${ }^{147}$ and complementary logic circuits, ${ }^{138}$ despite being underperforming compared to p-type OECTs.

2.3.2.1.2. Biointerfacing, Measurements, and Figures of Merit. Integration of cells with the OECT can either be direct or indirect. Examples of the latter include cells suspended within the electrolyte between the gate and the channel or supported by Transwell membranes located above the channel. ${ }^{271}$ Typically, the measurements performed to monitor cellular activity are transient, ${ }^{60}$ for example, bandwidth of the device (monitoring the $g_{\mathrm{m}}$ over a range of frequencies). For example, formation of biolayers, such as barrier tissue, ${ }^{274,275}$ or cell membranes, ${ }^{253}$ 

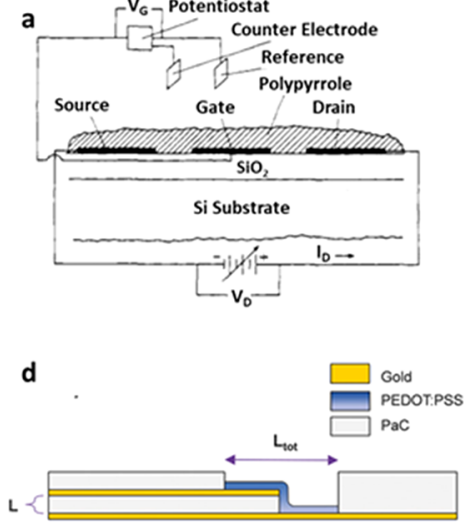

b

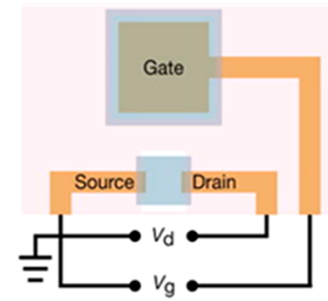

e

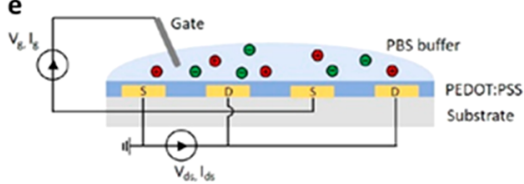

c

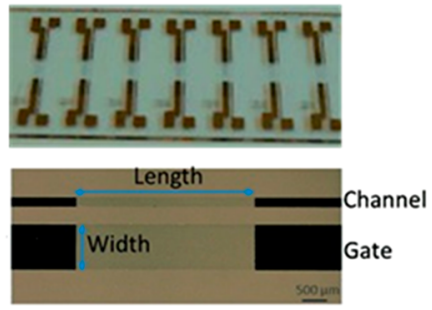

f

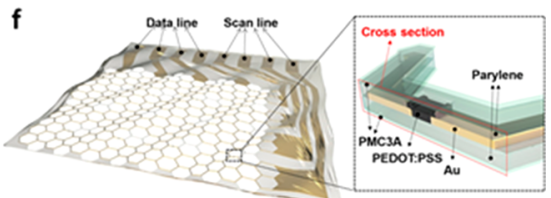

Figure 17. (a) Architecture of the first OECT device reported. Reprinted with the permission from ref 284. Copyright 1984 American Chemical Society. (b) OECT with PEDOT:PSS coated gold gates in plane with the channel fabricated on glass. Reprinted with permission from ref 288. Copyright 2021 AIP Publishing. (c) All PEDOT:PSS OECT, comprising a PEDOT:PSS channel and a PEDOT:PSS planar gate. Adapted with permission from ref 285. Copyright 2015 WILEY-VCH Verlag GmbH \& Co. KGaA, Weinheim. (d) Vertical OECT configuration. Reproduced with permission from ref 286. Copyright 2017 WILEY-VCH Verlag GmbH \& Co. KGaA, Weinheim. (e) Architecture of interdigitated OECTs gated with an external $\mathrm{Ag} / \mathrm{AgCl}$ gate. Reprinted with permission from ref 289. Copyright 2021 American Chemical Society. (f) OECT arrays fabricated on flexible/stretchable substrates. Reproduced with permission from ref 290. Copyright 2018 The Authors, some rights reserved; exclusive licensee American Association for the Advancement of Science. No claim to original U.S. Government Works. Distributed under a Creative Commons Attribution NonCommercial License 4.0 (CC BY-NC), https://creativecommons.org/licenses/by-nc/4.0/.

between the OECT channel and the gate affects the ionic flux reducing the bandwidth of the device (Figure 16e) and the cutoff frequency. Cut-off frequency is typically the figure of merit to characterize ion permeability of the biolayers (see section 2.4). The latter is a result of the physical barrier that cells form, which impedes the ion flow between the electrolyte and the CP channel. ${ }^{276}$ Similarly, this effect is pronounced in the response time of the device (i.e., how fast the drain current responds upon a gate pulse), which is another parameter or figure of merit to evaluate the biolayer quality (Figure 16d). ${ }^{275,277,278}$ Currentdriven OECTs were also developed recently for monitoring cell barriers in vitro. ${ }^{274}$ In this mode of operation, application of a voltage at the gate $\left(V_{\text {in }}\right)$ is used to control doping of the channel, and a current generator applies a current bias $\left(I_{\mathrm{b}}\right)$, allowing measurement of the output voltage $\left(V_{\text {out }}\right)$. Compared to stateof-the-art OECT devices, current-driven OECTs can overcome limitations related to voltage operation and ion sensitivity. The ability of such devices to tune the bias current greatly extends their operation window without inducing harmful redox reactions that can affect the adapted biological systems. This device operation approach has been already applied for the monitoring of barrier properties of cell layers ${ }^{279}$ and will benefit new developments in complementary OECT circuits. ${ }^{280}$

Importantly, the transparency of PEDOT:PSS channels provides the advantage to evaluate cells and tissue with simultaneous electrical and advanced optical measurements. ${ }^{253,281}$

2.3.2.1.3. OECT Architectures. Since their development in the $1980 \mathrm{~s},{ }^{284}$ organic electrochemical transistors (OECTs) have undergone a substantial transformation. The original OECT device architecture consists of an array of three gold electrodes (S-D-G) in plane with PPy as the channel (Figure 17a). To date, the most commonly used architecture comprises a CP channel between source and drain contacts with an external gate electrode immersed in the electrolyte solution, as illustrated in Figure 14. Another OECT architecture that is particularly interesting for in vitro studies is the planar gate OECTs, where the $\mathrm{CP}$ channel is microfabricated in plane with the gate (Figure 17b). The gate and channel dimensions can be tuned to maximize gating efficiency and current modulation of the channel. ${ }^{285}$ All PEDOT:PSS planar gate OECTs are also utilized, where both the gate and channel are made of PEDOT:PSS providing more flexibility during processing together with efficient gating (Figure 17c) ${ }^{285}$ Vertical organic electrochemical transistors, where the channel is sandwiched between the source and drain contacts vertically, were also demonstrated (Figure 17d). ${ }^{286}$ This OECT architecture reduces the device footprint and allows for dense arrays to be made without sacrificing transconductance. Interdigitated OECTs represent another interesting device architecture explored for in vitro applications. ${ }^{287}$ These devices feature superior $g_{\mathrm{m}}$ at small device areas compared with single channel OECTs, which can be tuned by changing the channel length and width and the number of electrode fingers (Figure 17e). Importantly, different deposition and patterning techniques on flexible and stretchable substrates demonstrate the unique form factors that can be achieved with these devices (Figure 17f).

2.3.2.2. Electrolyte Gated Field-Effect Transistors (EGOFETs). EGOFETs use a conjugated polymer (or small molecule) patterned between a source and drain contact and gated with an electrode immersed in aqueous electrolyte. Upon gating, electronic charges from the polymer semiconductor are driven by voltage at the interface with the electrolyte and electrostatically interact with ions. ${ }^{291}$ The accumulation of charges at the interface results in two electrical charge double layers: one at the channel/electrolyte and one at the gate/electrolyte interface with remarkably high double layer capacitance. ${ }^{292}$ This principle of operation is utilized to build ultrasensitive EGOFET-based biosensors able to detect single molecules of analyte. ${ }^{135,293}$ For the vast majority of EGOFETs used as biosensors, the figure of merit is the threshold voltage $\left(V_{\mathrm{th}}\right)$, which is the minimum $V_{\mathrm{GS}}$ needed to create a conducting path between the source and drain (i.e., turn the device ON). When, for example, a binding event occurs, $V_{\text {th }}$ shifts to either higher or lower values depending on the (bio)molecules added. Other figures of merit include the subthreshold swing, charge carrier mobility 
a
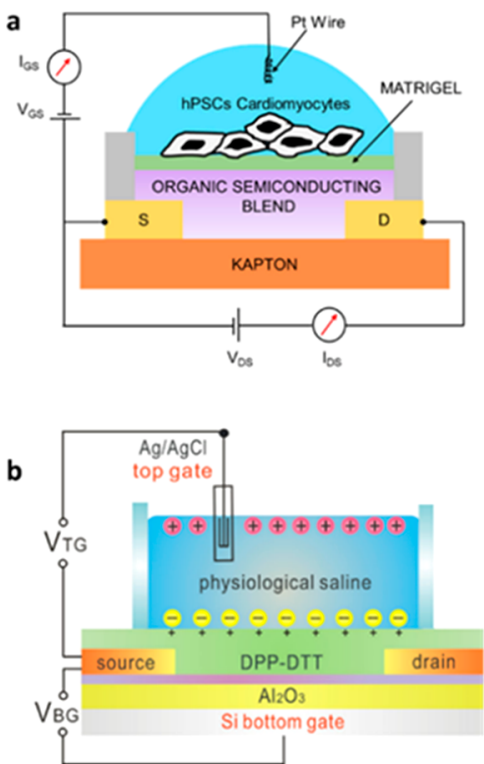

C

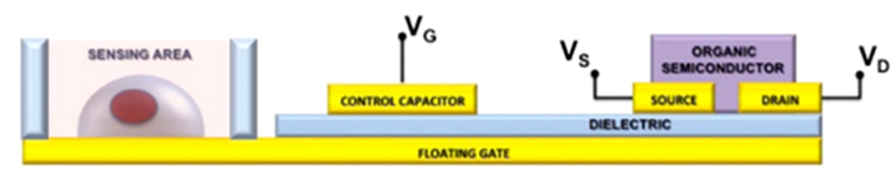

Setting the working point

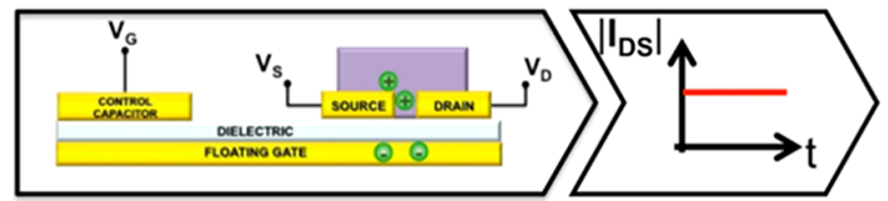

Recording cells' activity

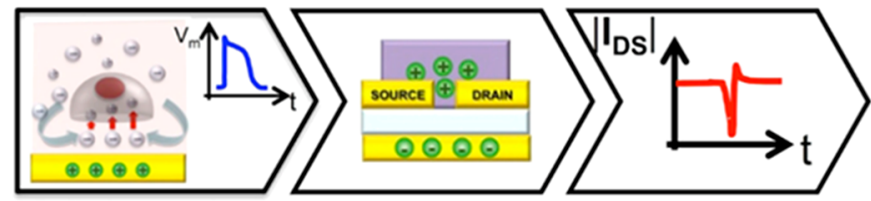

Figure 18. EGOFET device architectures used for in vitro studies. (a) EGOFET device used for in vitro studies of cardiomyocytes. Reprinted with permission from ref 304. Copyright 2020 Elsevier. (b) Double gate EGOFET used to study cells in vitro at low operation voltages. Adapted with permission from ref 302 Copyright 2017 American Chemical Society. (c) Device design and operation principles of organic charge modulated field effect transistors. Reprinted with permission from ref 57. Copyright 2015 The Authors, published by Springer Nature under a Creative Commons Attribution 4.0 International License, http://creativecommons.org/licenses/by/4.0/.

a

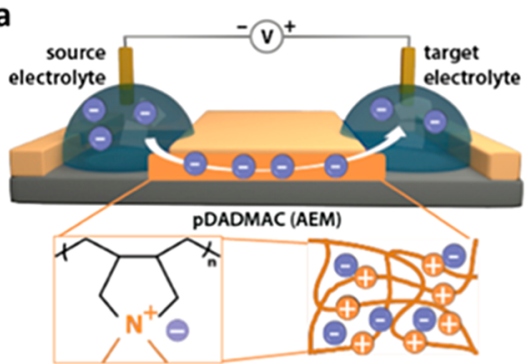

C

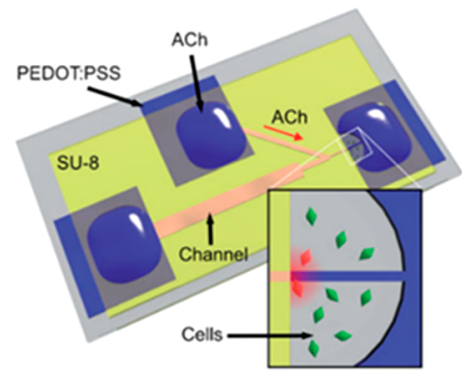

b

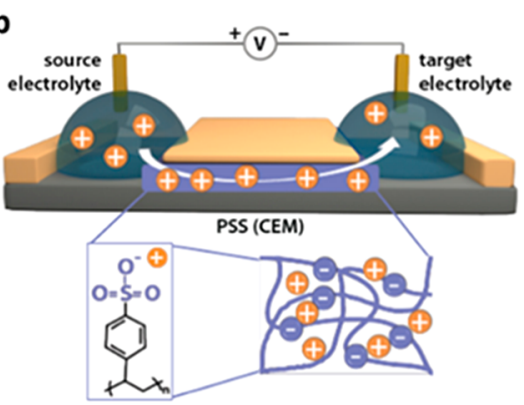

d

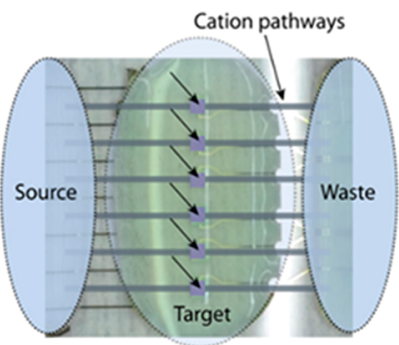

Figure 19. Schematic showing the device configuration and the working principle (a) of a potential ion selective OEIP and (b) a cation selective OEIP. Adapted with permission from ref 307. Copyright 2019 The Authors, published by IOP Publishing Ltd. under the terms of the Creative Commons Attribution 3.0 license, https://creativecommons.org/licenses/by/3.0/. (c) Schematic illustration of the OEIP. The PEDOT:PSS electrodes are connected by cation selective polyelectrolyte channels. The SU-8 layer serves as insulation and encapsulation layer with defined openings for the electrolytes. Reproduced with permission from ref 310. Copyright 2009 WILEY-VCH Verlag GmbH \& Co. KGaA, Weinheim. (d) Top view of an OEIP array with six individually addressed channels in parallel. Arrows point to six delivery points in a common target electrolyte. Reproduced with permission from ref 311. Copyright 2016 The Authors, published by AAAS under Creative Common Attribution-NonCommercial 4.0 International (CC BY-NC 4.0), https://creativecommons.org/licenses/by-nc/4.0/.

$(\mu)$, and transistor output current. ${ }^{294}$ For a comprehensive summary on EGOFET-based biosensors, we recommend other recent reviews. ${ }^{295,296}$

Despite their remarkable sensitivity to capacitance changes, the main limitation of EGOFETs as direct interfaces for in vitro bioelectronics is the relatively high voltage required for operation. Operation in aqueous, physiologically relevant electrolytes dictates voltage operation of $<11 \mathrm{Vl}$ to avoid electrolysis of water. In practice, the operational voltages must be as low as possible since CPs act as catalysts and facilitate 
a

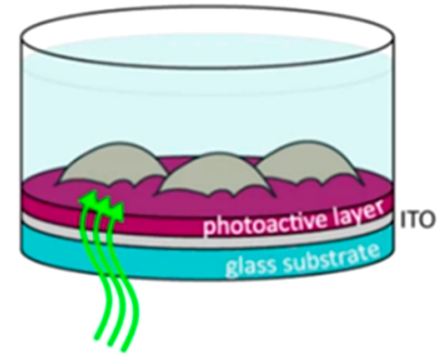

b

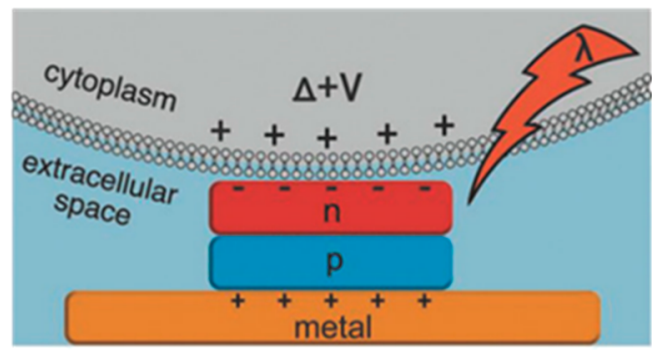

C

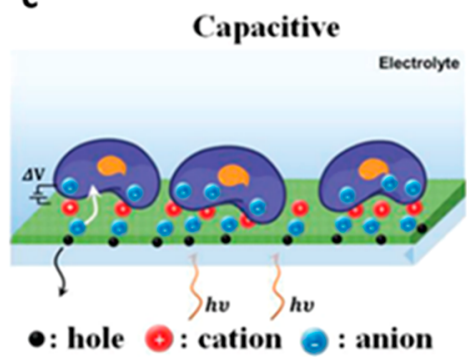

Faradaic
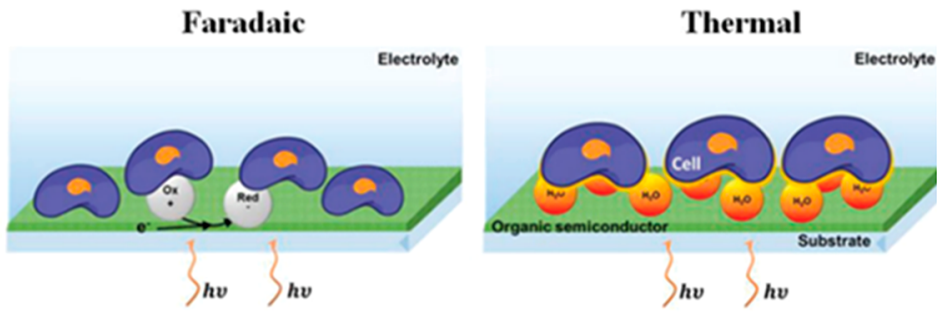

Figure 20. (a) Early photoactuation studies using a single semiconducting polymer film (P3HT) coated on ITO/glass substrates to stimulate neuronal cultures in vitro. Reproduced with permission from ref 321. Copyright 2015 The Authors, published by Springer Nature under a Creative Commons Attribution 4.0 International License, https://creativecommons.org/licenses/by/4.0/. (b) Photoactuators made of CPs in a $\mathrm{p}-\mathrm{n}$ junction configuration. Reproduced with permission from ref 322. Copyright 2018 WILEY-VCH Verlag GmbH \& Co. KGaA, Weinheim. (c) Mechanisms of photoactuation on cells mediated by an absorbing conjugated polymer. Reproduced with permission from ref 326 . Copyright 2019 WILEY-VCH Verlag GmbH \& Co. KGaA, Weinheim.

oxygen reduction reactions at voltages lower than $1 \mathrm{~V},{ }^{297}$ with severe impact on both cell culture and material stability. ${ }^{298}$ EGOFETs that operate below $1 \mathrm{~V}$ were successfully demonstrated; however, at this low voltage operation regime, the current and transconductance are significantly lower compared with OECTs. To overcome the limitations of high voltage operation, special device designs allow EGOFETs to take advantage of the ionic contributions to the overall current and operate in the voltage range below $1 \mathrm{~V} \cdot{ }^{299}$ Nevertheless, these devices are stable and compatible with cell culture conditions $^{300}$ and have been explored for in vitro bioelectronic studies. $^{266}$ The different EGOFET device designs and their exact mechanisms of operation were recently reviewed by Torricelli et al. ${ }^{299}$ A few examples are also given here. Kyndiah et al. proposed an EGOFET for recording the extracellular potential of cardiac cells, which was operated at $V_{\mathrm{G}}=-0.7 \mathrm{~V}$ and $V_{\mathrm{D}}=-0.5 \mathrm{~V}$. In addition, the device surface was coated with Matrigel to favor the adhesion of cardiac cells (Figure 18a). ${ }^{301}$ In another study, Zhang et al. reported a double gate transistor based on highly crystalline DPP-DTT CP channel. ${ }^{302}$ This device design, shown in Figure 18b, involves a gate immersed in the aqueous electrolyte that is controlled by an additional bottom gate separated from the gate dielectric. With this design, the threshold voltage of the channel can be tuned in a voltage window that is safe for the cells and used to monitor the detachment of human mesenchymal stem cells. Another approach to lower the operational voltage of EGOFETs was introduced by Desbief et al., who used EGOFETs with $\mathrm{Au}$ nanoparticles embedded on organic small molecule (i.e., pentacene $)^{303}$ to interface with neuroblastoma cells. A floating gate, interconnected with an EGOFET, on which cells are cultured can also be used. This device can be operated in liquid environments at low voltages without any external reference electrode and is termed an organic charge modulated FET
(OCMFET). ${ }^{266}$ Spanu et el. optimized this device architecture to record the electrophysiological activity of cardiac cells in vitro, and their device design and operation principles are shown in Figure $18 \mathrm{c} .^{57}$

2.3.3. Ion Pumps. Ion pumps operate as drug/therapy delivery devices transferring ions from one side of a membrane to the other, in analogy to ion pumps found in cell membranes. The organic electronic ion pump (OEIP) interconnects two isolated electrolyte regions through an ion exchange membrane to selectively deliver ions or charged molecules and stimulate cells locally. ${ }^{305}$ One electrolyte region contains the ion or drug to be delivered (the source), and the other electrolyte is the target, in which cells or tissue can be attached. ${ }^{306}$ When potential is applied between the two electrolyte reservoirs, ions are selectively delivered through an ion exchange membrane to the target electrolyte. A schematic of a typical OEIP setup and the operation principles are shown in Figure 19a,b. In this case, a polycationic membrane made of poly(diallyldimethylammonium chloride) $\left(\mathrm{pDADMAC}^{+}\right)$was used to selectively deliver anions and a polyanionic membrane made of $\mathrm{PSS}^{-}$was used to selectively deliver cations to the target electrolyte. $^{307}$

The ion delivery or drug dose can be finely tuned by the current run through the channels. ${ }^{308}$ Device design can also be tuned to regulate the delivery in a defined space within the target electrolyte. ${ }^{309}$ For example, Tybrandt et al. demonstrated an OEIP comprising PEDOT:PSS channels connected with a cation selective membrane to deliver acetylcholine, which will be discussed below in section 3.4.2 (Figure 19c). ${ }^{310}$ Furthermore, ion pumps can be constructed in arrays to deliver drugs and ions at multiple local points and at the same time. As shown in Figure $19 \mathrm{~d}$, Jonsson et al. designed an array of parallel structures and demonstrated the simultaneous delivery of neurotransmitters from 6 individually addressed outlets in parallel with a temporal 
resolution of $50 \mathrm{~ms}^{311}$ In this case, the cation-selective membranes were made of overoxidized PEDOT:PSS coupled with the anion-permselective quaternized poly(vinylbenzyl chloride) (qPVBC). Interestingly, it was shown that OEIPs can be also integrated with electrodes to chemically stimulate and sense the changes at the same local point of the culture ${ }^{308}$ or operate autonomously after being powered wirelessly by an integrated organic solar cell. ${ }^{312}$ OEIPs are more suitable for delivery of small ions that can penetrate the polyionic membranes such as protons, ${ }^{313}$ glutamate, ${ }^{314}$ potassium $^{315}$ and chloride. ${ }^{316}$

2.3.4. Photoactuators. Photoactuators are devices that modulate cell activity through optical stimulation mediated by organic semiconductors, with high temporal and spatial resolution. This concept led to some impressive results, including the restoration of vision in blind animals. ${ }^{317,318}$ In general, using exogenous functional materials to selectively manipulate cell activity is considered a far less risky approach compared to optogenetics. $^{319}$

These devices also attracted significant interest because of their simple setup, ${ }^{320}$ which has been illustrated by Martino et al. $^{321}$ and is reproduced in Figure 20a. Incoming light is transmitted through the transparent conducting substrate (glass/ITO), absorbed by the photosensitive CP (P3HT in this case) and converted into a cue, modulating activity of cells directly cultured on its surface. The photosensitive component can be also made with a CP layer stack (or organic conjugated small molecules) to form light sensitive $\mathrm{p}-\mathrm{n}$ junctions (Figure 20b). ${ }^{322}$ The exact mechanisms of photostimulation using organic semiconductors are still under investigation and so far, three major mechanisms are proposed: photocapacitive, ${ }^{323}$ photo(electro) chemical, $^{324}$ or photothermal stimulation ${ }^{321}$ (Figure 20c). Hence, CPs can convert light into a cue and can modulate the cell membrane potential of cells that are usually in direct contact with the semiconductors. However, decoupling photochemical and photocapacitive effects is complex and requires the design of controlled photo-electrochemical measurements. ${ }^{325}$ For more detailed explanation about the photostimulation mechanisms using organic photoactuators, we recommend reading the review by Hopkins et al. ${ }^{326}$

A large number of organic semiconductors can be used for photoactuation on cells, chosen mainly from the wide library of materials that have been successfully used in solid-state optoelectronic devices. These materials show distinct optical and electronic properties and can be used to tune the sensitivity of the device at different light wavelengths ${ }^{327}$ and intensities. ${ }^{328}$ The Głowacki group recently reported the development of $p-n$ junctions based on conjugated small molecules and used them for optical stimulation of single cells ${ }^{329}$ and neuronal cultures in vitro $^{322}$ through photocapacitive effects. Earlier pioneering studies from Lanzani and co-workers reported blends of organic semiconductors comprising the p-type $\mathrm{P} 3 \mathrm{HT}$ and n-type fullerene derivative $[6,6]$-phenyl- $\mathrm{C}_{61}$-butyric acid methyl ester (PCBM) to trigger action potentials in neurons upon light illumination. ${ }^{134}$ Interestingly, photoactuation of cells mediated by polymer semiconductors was found to modulate the behavior of nonelectrogenic cells. A light sensitive CP interface was demonstrated to modulate the cell membrane potential of astrocytes, ${ }^{330}$ as well as to regulate the fate of endothelial colonyforming cells through photochemical reactions. ${ }^{324}$ In addition to CP thin films, nanoparticles made of CPs have also been used to control cellular activity with light. Zangoli et al. used polythiophene-based nanoparticles functionalized with amines, to polarize the membrane of live HEK-293 under illumination. ${ }^{331}$ Finally, photoactuators based on CPs have been used to optically trigger the redox states of specific proteins, ${ }^{332}$ modulate the formation of reactive oxygen species in cell cultures, ${ }^{333}$ and control the intracellular redox equilibrium in model organisms. $^{334}$

\subsection{Modeling Organic Bioelectronic Devices for In Vitro Applications}

This section covers the various types of modeling that have been or can be applied for the design, analysis, and simulation of complex biological systems. We focus on the two predominant approaches, empirical modeling (data-driven) and analytical modeling (simulations), discussing the advantages and disadvantages of each approach and give examples on their use for (a) extracting information to better understand biological systems that are interfaced with organic electronic transducers and (b) studying the relationship between device operation, figures of merit, and physical parameters (such as geometry and material composition) to design bioelectronic devices with optimized performance for specific applications.

Data-driven approaches are typically referred to as regression. In practice, a model that corresponds to an equation relating the input to a measured output is proposed, where the unknown parameter values (proportionality constants, offsets, etc.) are varied in order to maximize the fit of the model to the experimental data. The most crucial aspect of this approach is the selection of the right model that will provide the relevant information on the nature of the system under investigation. It should be noted that different models, with different parameters, may yield similar fits. Hence, in order to enhance the reliability of the model, components or subunits that exhibit (inputoutput) behaviors that mimic known, decoupled physical phenomena are preferred. The most common approach to this is to model phenomena within the system as equivalent circuit elements. ${ }^{335}$ In contrast, elucidating relationships between device design parameters and device operation requires analytical derivations of the coupled physical processes within the system. While the empirical approach is useful for extracting information on the state of a system with a well-defined operating mechanism, this analytical approach allows for the operating mechanism to be better understood, thus informing the interpretation of empirically determined parameters or figures of merit and used to optimize device design. Deriving analytical expressions is, however, a challenging task, involving assumptions. To more accurately model complex systems, closed-form analytical expressions can be applied, such as finite element modeling (FEM) or finite difference modeling (FDM). Such simulations can enable interrogation of the relationship between device design and performance.

In the context of in vitro bioelectronics and CPs, which are mixed ionic and electronic conductors, the nature of the interface between ionic and electronic domains constitutes a core component in both types of models. To date, device modeling in the in vitro arena has been largely restricted to devices derived from ion-impermeable CPs, used in EGOFETs, and ion-permeable CPs (mixed conductors), used in OECTs, with both material types also used as electrodes. For the former, given that ionic flux is restricted to the electrolyte and electronic flux to the organic conductor, coupling between these domains is mediated by an electric double layer (EDL) at the conductor surface. In terms of CPs, however, there remains some debate as to the exact mechanism of interfacing, ${ }^{124}$ due to the mixed 
conduction properties exhibiting primarily capacitive behavior. ${ }^{106,110}$ Consequently, the majority of models currently employed toward in vitro applications revolve around this capacitive element.

2.4.1. Analytical Modeling. As stated above, analytical modeling has largely been restricted to improving understanding of the operation of organic transistors (see section 2.3.2), improving our understanding of the relationship between the operational parameters, figures of merit, and device geometry (and material).

2.4.1.1. Deriving the Figures of Merit of Organic Transistors. The primary figure of merit for a transistor is the quasi-static transconductance $\left(g_{\mathrm{m}}\right)$, which refers to the efficiency of the gate to modulate the channel conductivity, and is defined as

$$
g_{\text {m, quasi-static }}=\left.\frac{\partial I_{\mathrm{d}}}{\partial V_{\mathrm{g}}}\right|_{Q}=\left.\lim _{f \rightarrow 0} \frac{\partial i_{\mathrm{d}}}{\partial v_{\mathrm{g}}}\right|_{Q}
$$

where $I_{\mathrm{d}}$ and $V_{\mathrm{g}}$ are the steady state drain current and gate voltage, respectively, and $i_{\mathrm{d}}$ and $v_{\mathrm{g}}$ are the small signal drain current and gate voltage, respectively. $Q$ is the system operating point, defined as $\left\{V_{\mathrm{g}, \mathrm{DC}}, V_{\mathrm{D}, \mathrm{DC}}\right\}$. An additional figure of merit is the small signal bandwidth, or the cutoff frequency at which the transient transconductance $\left(g_{\mathrm{m}}=\partial i_{\mathrm{d}} / \partial v_{\mathrm{g}}\right)$ decreases by $3 \mathrm{~dB}$. The bandwidth of the transistor describes the fastest signal (highest frequency) that can pass through the system without being attenuated, and consequently, it reflects the fastest (biological) event that can be transduced by the sensor. A similar parameter is the system time constant $(\tau)$ or response time, which refers to the time taken for the system to respond to a step input. While the time constant is not limited to the small signal domain (unlike $g_{\mathrm{m}}$ ), it is also a measure of the system bandwidth reflecting the ability to respond to transient signals. It should be noted, that a system may have multiple time constants, referring to different physical processes as well as to different classes of system inputs (for example, OECTs have been known to exhibit nonsymmetrical time constants for rising and falling pulse inputs). ${ }^{301}$ Finally, owing to their central role in dictating sensor performance, important figures of merit are the (ion and hole) mobilities $(\mu)$ and the interfacial capacitance $\left(C^{*}\right)$. The primary difference between EGOFETs and OECTs from a modeling perspective is that the interfacial capacitance of an EGOFET is a function of the surface area of the CP exposed to the electrolyte, while that of an OECT is a function of the volume of the CP. Below we discuss the relationships between the figures of merit and device performance and the device geometry and material design criteria, which have been concluded from analytical and numerical modeling and simulation.

2.4.1.2. Modeling Organic Transistors. The most widely employed transistor configuration in bioelectronics is the OECT, described in section 2.3.2.1. While the popularity of this architecture has driven much of the modeling interest, an additional reason for the skew between OECT and EGOFET modeling efforts is the relative similarity of the EGOFET to classical transistors. In particular, the interfacial capacitance in an EGOFET arises from an EDL formed on the surface of the channel, which can be effectively modeled as a thin (on the order of nanometers), parallel plate capacitance isolating the gate from the channel, where the charge on the capacitance dictates the degree of doping/dedoping. ${ }^{336}$ Clearly, this is equivalent to the capacitance in metal oxide semiconductor field-effect transistor
(MOSFET) devices due to the polarization of the metal oxide dielectric, with the primary difference being the order of magnitude of the capacitance. OECTs, on the other hand, represent a greater modeling challenge, due to the mixed conduction of their CP channels and the distributed capacitive coupling present throughout the volume of the channel, which violates standard assumptions in transistor modeling, such as the gradual channel assumption. In particular, Kaphle et al. examine the ability of common CPs to conduct both ionic and electronic charge carriers within the polymer bulk. ${ }^{337}$ It is shown empirically and numerically (by way of finite element simulation) that this property results in the lateral mass transfer of ionic charge carriers along the channel, mediated by concentration dependent diffusion and local electric field dependent migration (drift). The ionic flux within the channel is therefore a function of the gate potential as well as the drain potential and the gate geometry and placement relative to the channel in space. This results in the concentration of ionic species within the $\mathrm{CP}$ varying nonlinearly along the channel, aggregating in proximity to the drain terminal, and resulting in a space charge concentration that is not solely a function of the gate potential and the channel potential at a particular lateral position, thus violating the gradual channel assumption. This being said, the gate capacitance in EGOFETs does depart from standard parallel plate capacitance as it is voltage dependent. Furthermore, while the (inner) Helmholtz layer dominates the capacitance and is well approximated by a parallel plate model, the diffuse layer, extending into the bulk of the electrolyte also contributes to the net capacitance; more recent modeling approaches have been employed to further examine the exact structure of the EDL. ${ }^{338}$

2.4.1.3. OECT Modeling. A model of seminal importance in understanding OECTs is the Bernards-Malliaras (B-M) model. ${ }^{339}$ The B-M model posits that the OECT consists of an ionic and an electronic circuit, where the ionic circuit is modeled as an electrolyte resistance in series with the coupling capacitance described above, while the electronic circuit is modeled as a polymer (hole) conductivity, which is a function of the charge on the coupling capacitor. The coupling capacitor is the electrostatic linkage between ionic flux in the ionic circuit and electron/hole drift in the electronic circuit, a noncommunicating interface that allows information to be shared between the two domains. The B-M model focuses on OECTs where the active material is PEDOT:PSS, and therefore the coupling capacitance in question takes the form of EDLs at the interface between the PSS template and the PEDOT crystallites. However, due to the relatively abstracted nature of the B-M model, the concepts may potentially be applied to OECTs fabricated using other active materials, where the principal difference is likely to be whether the coupling capacitance is a function of the active material volume (as in PEDOT:PSS) or it is exposed surface area. The B-M model conceptualizes the coupling function of the interfacial capacitance as occurring by way of displacement, a unit of charge accumulated on the coupling capacitor results in a hole (polaron) being displaced from the PEDOT bulk into the (gold) substrate, inducing an electronic current. The resulting decrease in hole density in the $\mathrm{CP}$ corresponds to a decrease in the electronic conductivity of the CP. In this way, ionic current is measurable as both electronic current and channel conductivity modulation, corresponding to the two primary uses of CPs as electrodes and transistors. As per the definition of capacitance, the charge accumulated on the coupling capacitor is a function of the 
magnitude of the capacitance (and hence the active material and geometry) and the potential difference developed across the capacitance. The potential across the capacitive interface is a function of the applied gate potential, with the two related by the first order dynamics of the ionic circuit, mediated by the ionic time constant, $\tau_{\mathrm{i}}$. Thus, the gate voltage is related to the channel conductivity by way of the ionic circuit transient dynamics, the geometry, and material composition of the system, which illustrates the reliance of the transistor action on these system properties that are linked to the system's figures of merit.

While various improvements and adaptations of the B-M model have been proposed, ${ }^{340}$ the most pertinent one for in vitro applications has been the understanding gained on the relationship of the device geometry to its performance. Specifically, an important aspect of the B-M steady state model, as discussed by Friedlein et al., ${ }^{269}$ is an analytical description of the (quasi-static) transconductance of the device. It is shown that

$$
g_{\mathrm{m}} \propto \mu_{\mathrm{p}} C_{\mathrm{i}}^{*}\left(\frac{W d}{L}\right)
$$

where $C_{i}^{*}:=C_{\text {interface }}^{*}$ is the (channel) capacitance per unit volume, $W, d$, and $L$ are the channel dimensions, and $g_{\mathrm{m}}$ is the quasi-static transconductance. Further empirical investigations have shown that

$$
\left\{\begin{array}{l}
C_{\mathrm{i}} \propto W d L \\
R_{\mathrm{s}} \propto \sqrt{W L}
\end{array}\right.
$$

which implies that ${ }^{269}$

$$
\tau_{\mathrm{i}} \propto d \sqrt{W L}
$$

The first important deduction from this set of proportionalities is that there exists a trade-off between the device transconductance and the response time (mediated by the time constant, $R_{\mathrm{s}} C_{\mathrm{i}}^{*}$, of the ionic circuit), which is a proxy for bandwidth. The second deduction of note is the dependence of the transconductance on the factor $\mu_{\mathrm{p}} C_{\mathrm{i}}^{*}$, which refers to the dependence of the device on both the electronic and ionic circuits and, consequently, the material properties of the system. $^{123}$

As an addendum to the above, recent efforts have been made to explicitly model the dual conduction of both polarons and ions within the polymer bulk, thus incorporating both the drain current components due to ionic mass transfer within the polymer and the nonuniform spatial distribution of ions within the channel, which yields spatially heterogeneous charging of the interfacial capacitance. Szymański et al. ${ }^{341}$ simulated the device in two dimensions and concluded that the B-M model constituted an approximation for the system at low gate-channel potential differences, with deviating behavior above a potential difference of $\sim 0.1 \mathrm{~V}$. The so-called drift-diffusion equations have been used most recently by Paudel et al. ${ }^{342}$ who demonstrated that, when parasitic contact resistances were considered, the geometric scaling was linear for small values of length and depth but eventually saturated.

$$
g_{\mathrm{m}, \max } \propto 1-\mathrm{e}^{W d / L}
$$

2.4.1.4. Design Criteria for Efficient In Vitro Bioelectronic Devices. The modeling approaches outlined above relate device geometry, material, and modes of operation to the electronic behavior of the device and thus allow for rational design of systems used to interrogate or actuate biological systems in vitro. In addition to this, analytical models may be used to better conceptualize (and therefore improve) the mode of transduction. In particular, the approach to using CP devices in vitro is to incorporate the biological system into the bioelectronic device, such that the state of the biological system alters the device operation. Given that biological systems exist exclusively in the ionic domain, transduction must necessarily occur by designing the transducer so that the state of the biological system is coupled to the (capacitive) mechanism that couples the ionic and electronic domains in mixed conductors. In other words, the biological system state must either affect the ability of ions to compensate charged dopants within the polymer bulk or modulate the efficiency with which the gate induces ionic mass transfer into and out of the polymer. Given that biological species can generally not infiltrate the polymer bulk, the latter is the generally adopted approach. Examples of this approach commonly include the introduction of cell layers or cell components (membranes) that yield a region within the electrolyte altering permittivity and ionic impedance, modulating mass transfer, ${ }^{343}$ and the catalysis of biochemical reactions at the gate and drain electrode surfaces, which modulates the electrode-electrolyte interfacial potential. ${ }^{344}$ The impact on gating efficiency is modeled for the introduction of cell layers or membranes as variations in the capacitance of the gate or the impedance of the electrolyte solution, ${ }^{343}$ while modification of interfacial potentials can be modeled as variations in the effective applied gate potential. ${ }^{344}$

A third approach to coupling biological state to gating behavior is through biologically induced ionic mass transfer wherein the depolarization of the membranes of electroactive cells modulates local ionic concentrations, resulting in increased ionic chemical potential and hence diffusion. ${ }^{61}$ While this approach does not influence the efficiency of any physical gate, the biological system can be modeled as a virtual gate with varying applied gate potential. ${ }^{344}$

2.4.2. Empirical Modeling In Vitro. As discussed previously, equivalent circuit modeling should largely be considered a data-driven approach, wherein the impedance function of the chosen model is projected onto the data, in order to determine the circuit parameters that will yield an impedance that best fits the data. A difficulty with this approach, as mentioned above, is the ambiguous nature by which different circuit elements contribute to the net impedance; to circumvent this, a common workflow is to first model the acellular component of the system and fit the relevant parameters from reference data taken prior to the inclusion of the biological component of the system. Then, by making the assumption that the incorporation of a biological (sub)system does not change the impedance characteristics of the acellular system, one may fit the modeling parameters of the biological system from new impedance data, while keeping the parameters of the acellular component of the system constant.

2.4.2.1. Equivalent Circuit Modeling. Given the above, it is pertinent to first consider the generic modeling considerations for the acellular and then the biological components of bioelectric systems. In EIS (and electrical-cell impedance spectroscopy, ECIS), the acellular components of the system that contribute to the overall impedance are generally the electrodes and the electrolyte itself. As in the B-M model ${ }^{339}$ (discussed above), the electrolyte impedance can generally be modeled as a simple resistance. ${ }^{345}$ The electrodes, however, display an impedance that is a function of the electrode- 


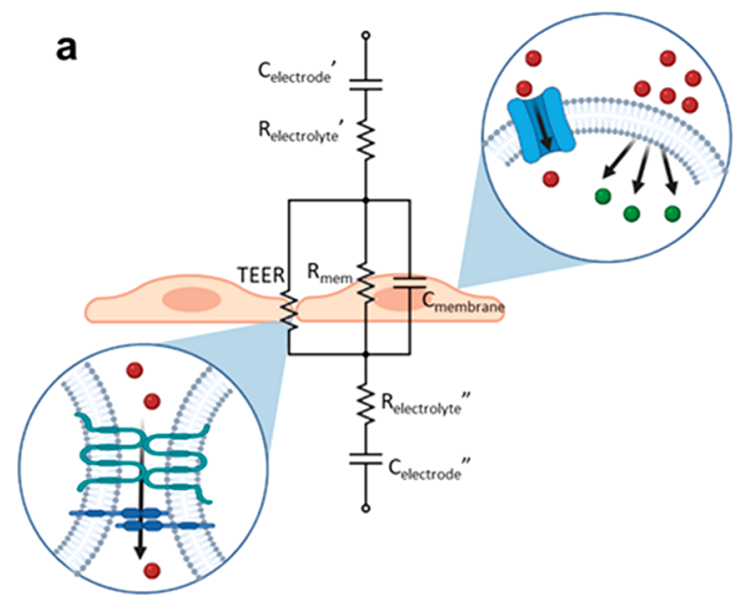

b

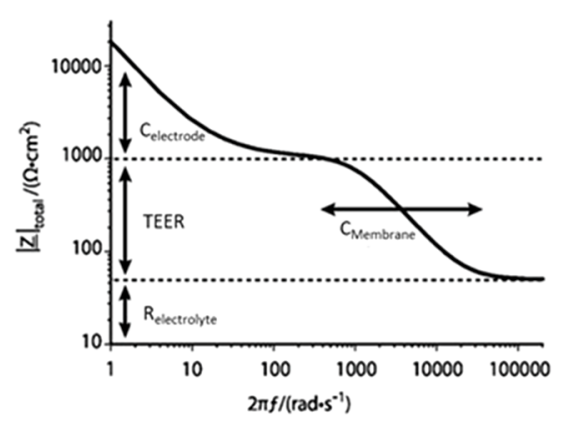

C

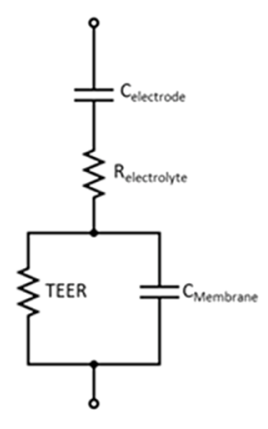

Figure 21. Impedance of a cell layer is determined by ionic flow via the paracellular and transcellular pathways. (a) The paracellular pathway is characterized by tight intercellular junctions, which impede ionic flow resistively. Similarly, transmembrane ion channels modulate membrane impedance resistively as a function of activation and expression. The decreased permittivity of the cell membrane allows for charge separation, modeled as a capacitance. (b) General form of the magnitude of the impedance of the standard circuit. The differing magnitudes and positions of the four elements results in a frequency domain decoupling, making it possible to extract parameter values. Adapted with permission from ref 349 . Copyright 2013 BioMed Central Ltd. under Creative Commons Attribution 2.0 Generic License, https://creativecommons.org/licenses/by/2.0/. (c) The electrode and electrolyte impedances are confounded; similarly, the membrane resistance is significantly larger than the TEER. The so-called standard model approximates the true equivalent circuit accordingly.

electrolyte interface and therefore depends on the charge separation, mass transport, and charge transfer phenomena occurring at the electrode surface. ${ }^{346,347}$ Ideally, nonpolarizable reference electrodes should be used for measurements that have a constant potential drop across the electrode-electrolyte interface. As such, in most cases, pseudo-reference electrodes, such as $\mathrm{Ag} / \mathrm{AgCl}$, are used and generally modeled as simple resistance. For long-term biological experiments, however, $\mathrm{Ag}$ / $\mathrm{AgCl}$ has been shown to be toxic for cells, ${ }^{335}$ so it is common to make use of polarizable electrodes, such as platinum or stainless steel, which electrically couple to the electrolyte by way of EDL formation. Similarly, as discussed previously, when CPs are used, the interface between the ionic and electronic domains is considered to be capacitive in nature. Typically, such components of the (electrode) system are modeled as contributing a (series) capacitive impedance. In terms of the biological model and its impedance, while certain bioelectronic applications involve functionalization of an electrode surface, which alters the electrode-electrolyte interface and hence the electrode impedance upon interaction with a bioanalyte, ${ }^{343,344}$ others involve introducing components of cells, such as cell membranes ${ }^{276,348}$ or cell layers, ${ }^{349,350}$ into the ionic domain of the system. The presence of the biological model alters the ionic flow through the electrolyte, manifesting a measurable impedance. In particular, the impedance is a measure of the restriction to ionic flux through a membrane or between adjacent cells. For cell layers, the former is known as the transcellular pathway and the latter as the paracellular pathway, as shown in Figure 21.

The impedance of the transcellular pathway is exclusively a function of the membrane permittivity and conductivity, both of which are significantly less than that of the interfacing electrolyte. This necessarily yields a restriction to ionic flow and a charge separation across the membrane, which is modeled as a parallel resistance and capacitance, respectively. Naturally, for whole cells, both the top and the bottom membrane contribute such a parallel combination to the net impedance; ${ }^{351}$ however, these two parallel circuits, which are effectively connected in series via the intracellular compartment, are heavily confounded, and so the model is generally reduced to a net parallel resistance and capacitance. Indeed, measurement of discrepancies in polarized cells can only be achieved by introducing a significant separation in the time constants of the two parallel combinations, generally by introducing an ion channel modulator into the apical compartment of the electrolyte. ${ }^{335}$ The paracellular pathway is characterized by intercellular junctions, which regulate the ease of ionic flow between cells. This impedance is modeled as purely resistive and is commonly referred to as the trans-epithelial/endothelial electrical resistance (TEER). ${ }^{335,345}$ Given that the transmembrane (ion-channel) conductivity is generally significantly greater than the TEER, the net impedance of the biological component is modeled as the TEER in parallel to the membrane capacitance, normalized by model area.

It can therefore be seen that the impedance of both cell membranes and tissue or barrier layers can be modeled as a resistance in parallel to a capacitance, which is in series with the impedance of the electrodes within the system and the electrolyte. In both instances, the capacitance is a reflection of the membrane permittivity (although for complete cells this is the amalgamation of the apical and basal membrane capacitances). However, for cell layers, the parallel resistance models the impedance to ionic flux between cells (TEER), while in cell membranes, the parallel resistance models the impedance to ionic flux through the membrane via the presence of defects (i.e., ion channels) (Figure 22a). Thus, while modeling conceptually different aspects of the biological systems, the net model, shown in Figure 22a, is mathematically identical and is the most commonly used model. Thus, this model shall henceforth be referred to as the standard model. ${ }^{335,345,349}$ The standard model has been used to extract information regarding the temporal evolution of both cell membranes and tissue barrier models from typical experimental data such as those shown in Figure 22b. In particular, specific examples of applications to membrane models are provided in section 3.1 and examples of 

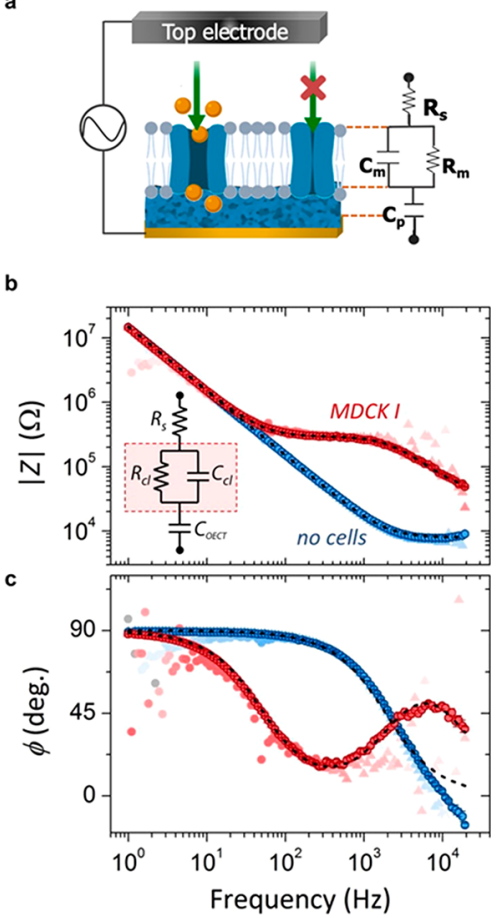

Figure 22. The standard model is applied to both lipid bilayer and tissue barrier models; transistor devices allow for increased sensitivity, alternate measurement modality, and low frequency resolution. (a) A lipid bilayer represents the transcellular impedance pathway in isolation. The resistive element is therefore the membrane resistance and not the TEER. Further, the capacitance in this instance is the channel capacitance of the $\mathrm{CP}$ and not the EDL capacitance of an electrode. Adapted with permission from ref 375. Copyright 2020 American Chemical Society. (b,c) Typical example of EIS data on CP electrodes covered with barrier tissue. This particular study combined the transconductance-derived impedance (from the OECT measurement) for the low frequency range with the EIS measurement (from electrode measurement) for the high frequency range yielding a noisefree wide impedance spectrum. Adapted with permission from ref 352. Copyright 2015 AIP Publishing.

applications to cell layer or barrier models are discussed in section 3.2.2.

\section{INTERFACING ORGANIC BIOELECTRONICS WITH CELLS}

This section covers the state-of-the-art in vitro organic bioelectronic applications for monitoring and controlling cell cultures (mammalian and bacterial), starting from simple cell membrane models to the typical 2D cell layers to complex 3D cell cultures, and finally to organotypic cultures. Advances for in vitro stimulation of cell cultures are also discussed.

\subsection{Cell Membrane Models}

The plasma membrane, also known as the "gatekeeper" of the cell, is fundamental for studying interactions of cells with compounds at the first point of contact. Developing cell-free biointerfaces that mimic plasma membranes is central for a range of applications of in vitro bioelectronics and for drug screening. ${ }^{353}$ The structure of the plasma membrane resembles a mosaic of components, the main constituent being a phospholipid bilayer that separates the internal volume of the cells and preserves their chemical content, necessary for biochemical functioning. ${ }^{354,355}$
Biomembrane mimetic systems can be purely synthetic (lipidonly), purely natural, or a combination of both (Figure 23). Integral membrane proteins provide directed transfer of molecules, sometimes with simultaneous transfer of ions in the same or opposite direction. ${ }^{356}$ Such transmembrane pores act as ion channels, membrane transporters, and pumps and can be switched on and off by external stimuli, including interactions with molecules or changes in $\mathrm{pH}$ or electric potential. Apart from water, oxygen, and carbon dioxide that routinely pass directly through the phospholipids in the cell membrane, larger molecules including glucose, as well as drugs and pathogens, may interact with the cell membrane and modulate ion channel activity or enter the cell. ${ }^{353}$ Most of these functions can be sufficiently reproduced by introduction of synthetic analogues mimicking basic recognition properties of membranes. Hence, the structure and composition of the cell membrane has often been re-created in vitro, serving as an excellent simplistic model to investigate cellular processes that occur at the surface of cells. As most of those processes impact the overall ion permeability of the membrane, a sensor system able to detect such changes is a valuable tool. ${ }^{357}$ Combining such membrane models with devices that are efficient ion-to-electron transducers, such as $\mathrm{CP}$ based devices, can offer unprecedented opportunities for sensing membrane interactions that rely on ion exchange, translating ionic signals to electronic ones. ${ }^{358}$

One of the simplest cell membrane models is unilamellar vesicles, $^{359}$ which are spherical phospholipid bilayers that typically consist of a single lipid composition with or without incorporated proteins or originate from the surface of the cells and hence encompass all the native components. Lipid monolayers ${ }^{360}$ represent another simplistic membrane model where the two bilayer leaflets are decoupled from each other, allowing the study of biological interactions at an aqueous/oil interface. They are typically highly insulating as they are defectfree.

In line with the use of simplified membrane models to quickly and massively assess the effect of drugs, lipid monolayers (made solely from lipid molecules) could play an integral role both as an easily scalable system and as a system where the effect of drugs (or other compounds) on the lipids of the lipid bilayer can be easily discerned. Recently, a lipid monolayer was stabilized in a liquid-liquid interface, a system that is sought to be stable and robust, in a novel organic transistor-based device configuration, the liquid-liquid phase separated (LiPS) OECTs (see section 2.3.2) (Figure 24a). ${ }^{267}$ The resulting platforms were successfully used to study the interaction of a mammalian model membrane with drugs ${ }^{361}$ and the disruption of a bacterial membrane model by an antimicrobial peptide (Figure 24b). ${ }^{362}$ Direct transduction of ion flux through the impermeable lipid monolayer (as a result of disruption or destabilization) was possible by recording the electronic current passing through the device, allowing the study of isolated membrane processes that do not rely on membrane proteins to be studied. ${ }^{362}$

The major applications of protein studies are based on lipid bilayer structures, where functional membrane proteins can be incorporated to study cellular phenomena in a controlled fashion. The experimental models of choice for studying such biomolecular interactions are planar lipid bilayers, which are mostly accessible to surface-sensitive techniques allowing integration with various technologies for their characterization.

Black lipid membranes, BLMs, first developed by Mueller and co-workers, are planar lipid bilayers supported in porous filters. Given the high electrical sealing properties of this model, they 


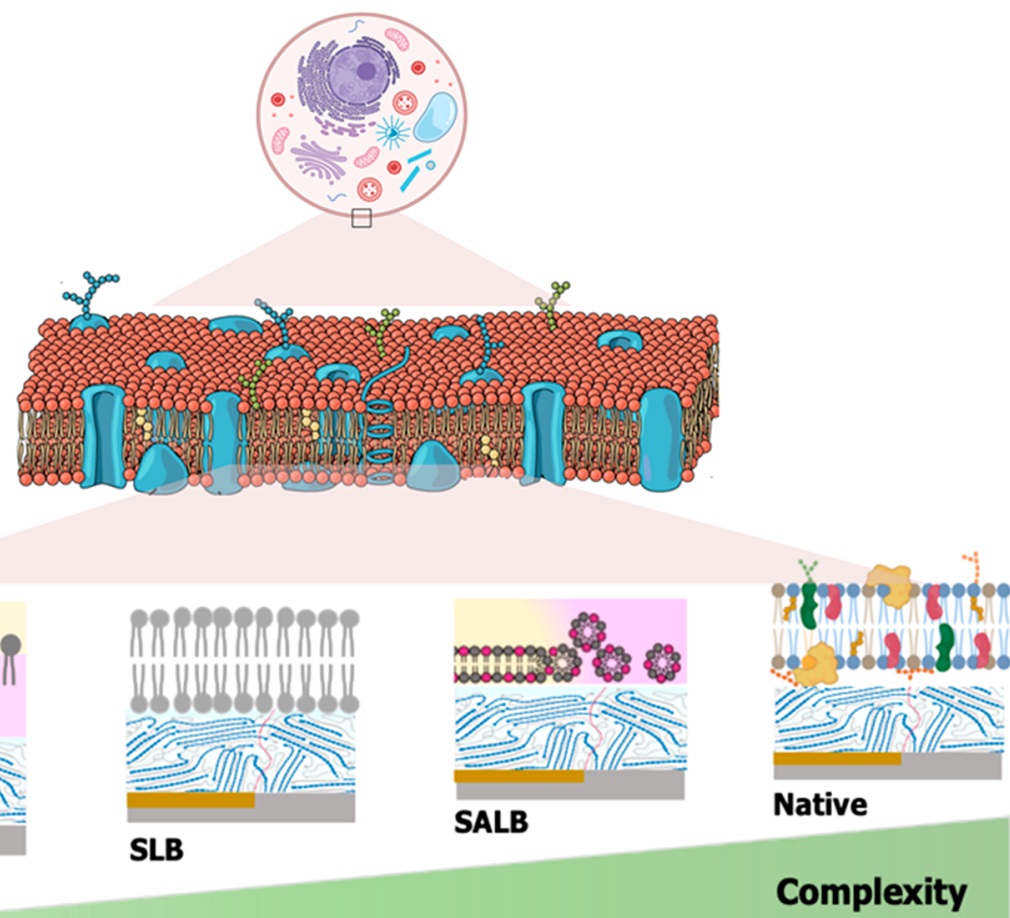

Figure 23. Schematic of the mosaic cell membrane model showing the complexity of the plasma membrane (containing proteins and glycosylated molecules) and the various cell membrane mimics (shown in increased biological complexity from left to right) that have been interfaced with organic bioelectronic materials and devices. Created with BioRender.com.

are highly suitable for ion channel studies. ${ }^{363}$ BLMs have been also extensively used to investigate pore forming peptide insertion into the phospholipid membrane. ${ }^{364}$ Malliaras et al. proposed a simple platform using an organic electrochemical transistor to incorporate BLMs and characterize the ion impermeable layer indirectly via the gating efficiency of the device, which would drop in the presence of an intact layer and partially recover when gramicidin ion channels were added ${ }^{276}$ (Figure 25a). Despite the highly insulating nature of BLMs, their major drawback is their limited stability as they remain functional for only few hours. Supported lipid bilayers, SLBs, pioneered by McConnell and colleagues in the 1980s, entered the field as a better biomimetic alternative to BLMs offering more robustness and stability and the possibility for long-term studies. ${ }^{365}$ SLBs are made by constructing 2D hydrated lipid films on an underlying solid surface. In SLB characterization, the standard optical methods used are fluorescence recovery after photobleaching (FRAP) and total internal reflection fluorescence (TIRF) microscopy for characterizing the bilayer fluidity or ordering of lipids or proteins in the bilayer, respectively. ${ }^{366,367}$ Although crucial in ensuring appropriate quality, coverage, and orientation of bilayers, these techniques do not offer an opportunity to conduct extensive quantitative analysis, that is, ion channel activity or high-throughput sensing capabilities. Planar SLBs supported on top of electronic transducers have been readily characterized with methods such as EIS and more recently transistor-based methods, providing quantitative analysis of transmembrane protein function in SLBs. ${ }^{368}$ As in whole cell studies, EIS models represent the bilayer using capacitors and resistors in series or parallel allowing the straightforward analysis of the response induced by membrane defects. Transistor technology also provides a quantitative means for characterizing SLBs due to their ability to induce or control the gating of the device. Early attempts at incorporating transistors with SLBs for monitoring membrane interactions were carried out by Torsi and her team using a P3HT field effect transistor and a phospholipid bilayer atop the organic semiconductor. The device was used to assess the effect of anesthetics on the permeability of the membrane (Figure 25b). ${ }^{369}$ Later, the same team showed that by coupling the channel of the transistor with a lipid bilayer bearing biorecognition elements they could easily detect membrane binding events. ${ }^{370}$

Increasing the complexity of SLBs to study membrane related events introduces challenges related to transmembrane protein stability and function. The proximity of the lipid bilayer to the solid support has typically hindered the diffusion of membrane components through the fluid bilayer, as sometimes the soluble domains of transmembrane proteins interact with the solid supports. Hence, substrates that retain the membrane's native environment and its fluidity and enable the investigation of both equilibrium and dynamic processes by allowing the mobility of lipids and membrane-incorporated proteins are in high demand. One promising strategy is the use of polymers that swell in aqueous media creating deformable and mobile substrates that faithfully recapitulate the cytoskeleton of cells. Recently, such polymer-supported membranes have been realized on top of CPs, providing mechanical matching with the soft biological structures while preserving their biological activity. In particular, PEDOT:PSS, owing to its swelling capacity, acts as a polymer cushion facilitating the mobility of transmembrane proteins and preserving a fluid membrane environment. Moreover, its inherent optical transparency allows multimodal transduction, combining fluorescence microscopy, and electrical measurements. The first attempt at interfacing SLBs with PEDOT:PSS configured both as electrode and OECT was with archaeal lipids allowing the activity of a typical pore forming toxin $(\alpha$ hemolysin) to be monitored, despite the relatively poor quality 
a
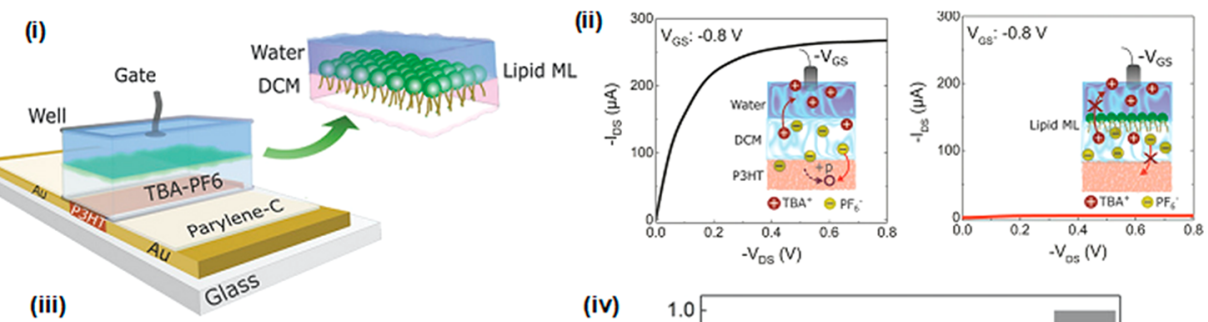

(iii)

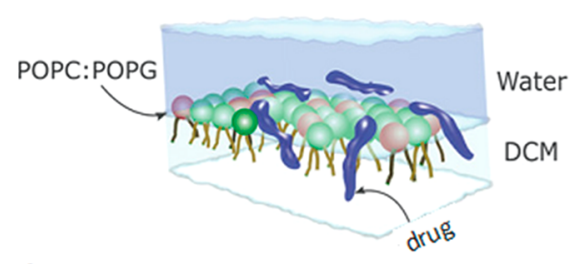

b

(i)

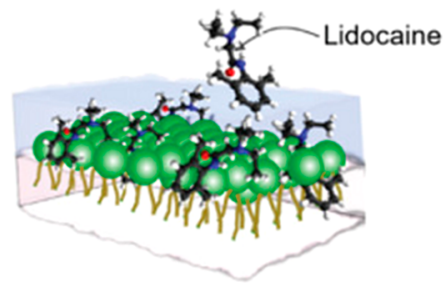

(iv)

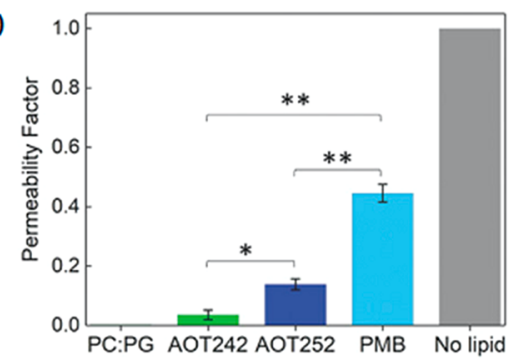

(ii)

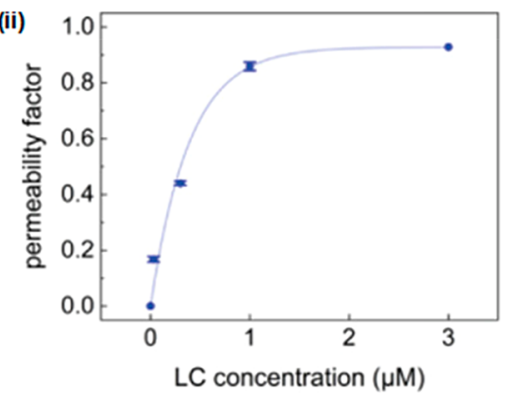

Figure 24. LiPS OECTs for high throughput drug screening. (a) (i) Schematic of the accumulation mode P3HT LiPS OECT structure stabilizing a lipid monolayer at the oil/water interface and (ii) IV response of the OECT $\left(V_{\mathrm{GS}}=-0.8 \mathrm{~V}\right)$ before and after monolayer formation showing the ion blocking effect of the lipid monolayer on the device. (iii) Schematic of the bacterial mimic (POPC/POPG) monolayer formed and antibacterial drug addition and (iv) comparative graph showing the ion permeability factor of the device after the addition of various antibacterial compounds. Adapted with permission from ref 362. Copyright 2018 WILEY-VCH Verlag GmbH \& Co. KGaA, Weinheim. (b) (i) Schematic of the mammalian lipid monolayer mimic when the anesthetic lidocaine is added and (ii) the membrane ion permeability for different lidocaine concentrations. Adapted with permission from ref 361. Copyright 2019 WILEY-VCH Verlag GmbH \& Co. KGaA, Weinheim.

a

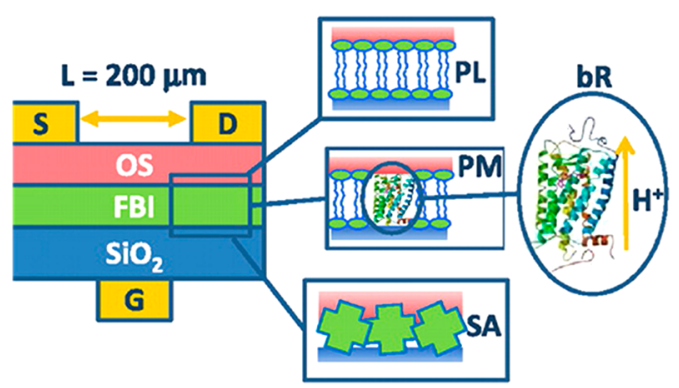

b

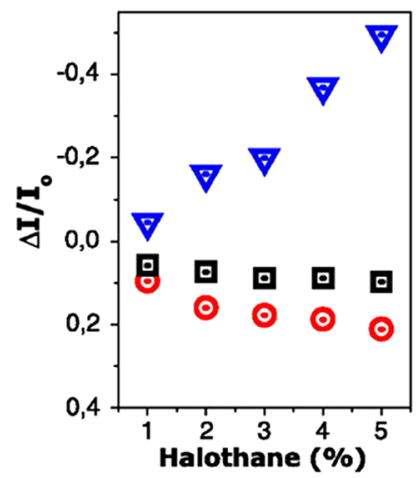

Figure 25. Functional biolayer (FBI)-OFETs. (a) Schematic illustrations of the FBI-OFET and the three different FBI structures showing that the phospholipid membranes are stabilized between the $\mathrm{P} 3 \mathrm{HT}$ active layer and the $\mathrm{SiO}_{2}$. (b) FBI-OFET as bioelectronic sensor of biological activity showing the calibration curves of the OFET-FBI responses to clinically relevant halothane concentrations (symbols: blue, lipid only membrane; red, purple membrane; and black, control). Adapted with permission from ref 369. Copyright 2012 National Academy of Sciences.

(in terms of membrane electrical properties) SLB formed. ${ }^{371}$ This study illustrated that although hydrated and mechanically compliant, the CP surface roughness in combination with its surface charges complicated the formation of SLBs, hitherto believed to form optimally on smooth rigid surfaces. The vesicle fusion method, in which small unilamellar vesicles spontaneously fuse and break, forming SLBs upon contact with a solid substrate, despite its simplicity and straightforwardness, poses challenges for many types of substrates. ${ }^{372}$ A follow-up study showed that the composition of the CP mixture prior to film formation, that is, the amount of ethylene glycol (EG) added, plays a significant role in the quality of the SLB formed, presumably due to the altered surface properties. ${ }^{373}$ Overcoming the surface-related limitations typically encountered when using the vesicle fusion method, a surface-agnostic approach to forming SLBs was introduced by Hohner et al. ${ }^{374}$ 
a
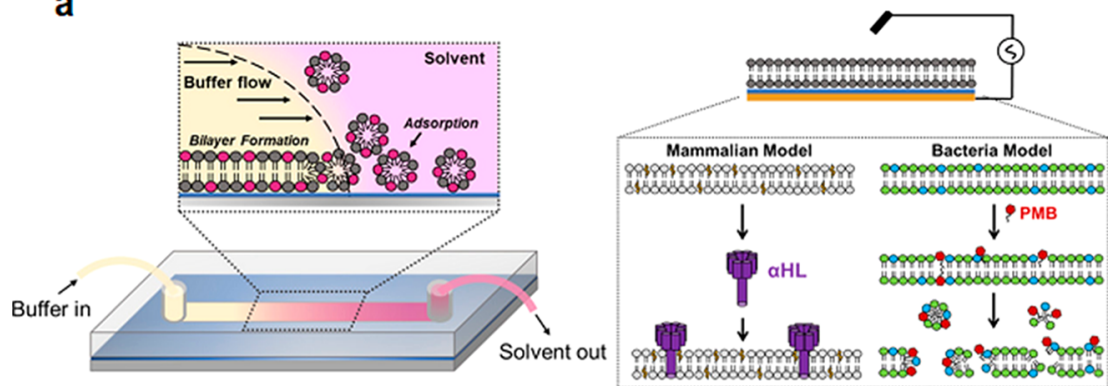

b
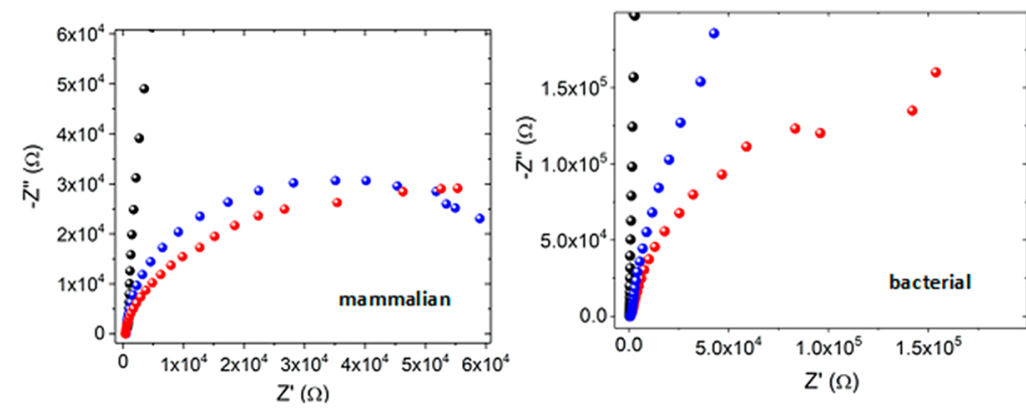

Figure 26. SALB formation on PEDOT:PSS devices. (a) Schematic showing the formation of the SALB inside a microfluidic channel showing the solvent phase containing lipid micelles doped with Texas Red labeled lipids (magenta) and the aqueous buffer flowing in to replace the solvent and forming the supported bilayer at the channel surface in the process (yellow phase). EIS configuration of the device and the SLBs, both mammalian and bacterial mimics, with the toxin $(\alpha-\mathrm{HL})$ and the drug (PMB) used to disrupt them, respectively. POPC and cholesterol comprise the mammalian bilayer, and POPE and POPG comprise the bacterial bilayer. (b) Nyquist plots from the EIS response of the SLB coated PEDOT:PSS electrodes, after their formation (red) vs pristine (black) and after the addition of the respective compounds (blue). Adapted with permission from ref 376 . Copyright 2019 American Chemical Society.
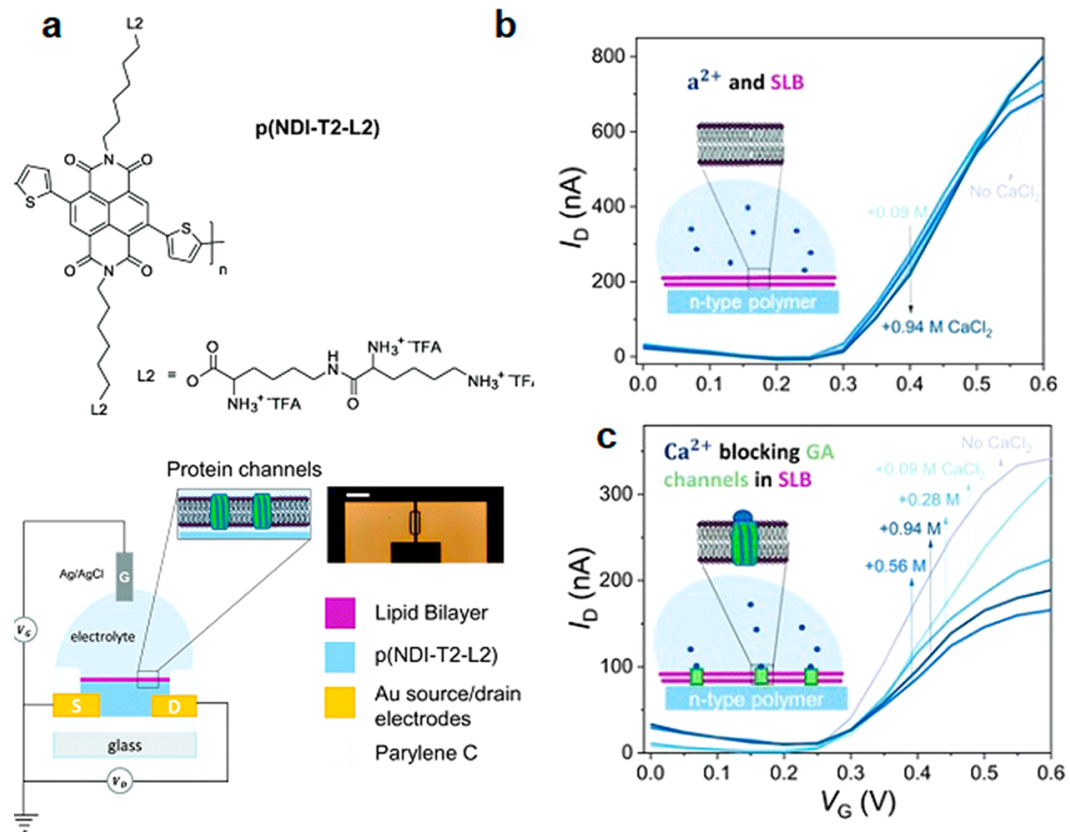

Figure 27. Measuring ion-channel activity on SLB functionalized n-type OECTs. (a) Chemical structure of the n-type lysinated semiconducting polymer $\mathrm{p}$ (NDI-T2-L2), where L2 corresponds to the lysine-based side chains with TFA being trifluoroacetic acid and schematic of the accumulation mode OECT incorporating the SLB. Transfer curves in the presence of different concentrations of $\mathrm{CaCl}_{2}$ for (b) pure DOPC SLB integrated OECT and (c) DOPC bilayer containing gramicidin pores. Insets are schematics of the SLB with integrated channel pores (gramicidin) on the n-type channel. Adapted with permission from ref 378. Copyright 2020 Royal Society of Chemistry under a Creative Commons Attribution- Non Commercial 3.0 Unported License (CC BY-NC 3.0) License, https://creativecommons.org/licenses/by-nc/3.0/.

and has been extensively investigated by the Cho team. ${ }^{375}$ Another advantage of the so-called solvent-assisted lipid bilayer (SALB) method is that it offers the possibility to add complex lipid compositions such as high cholesterol content, on a variety of substrates. SALB has been recently employed to prepare mammalian and bacterial model membranes using phospholi- 

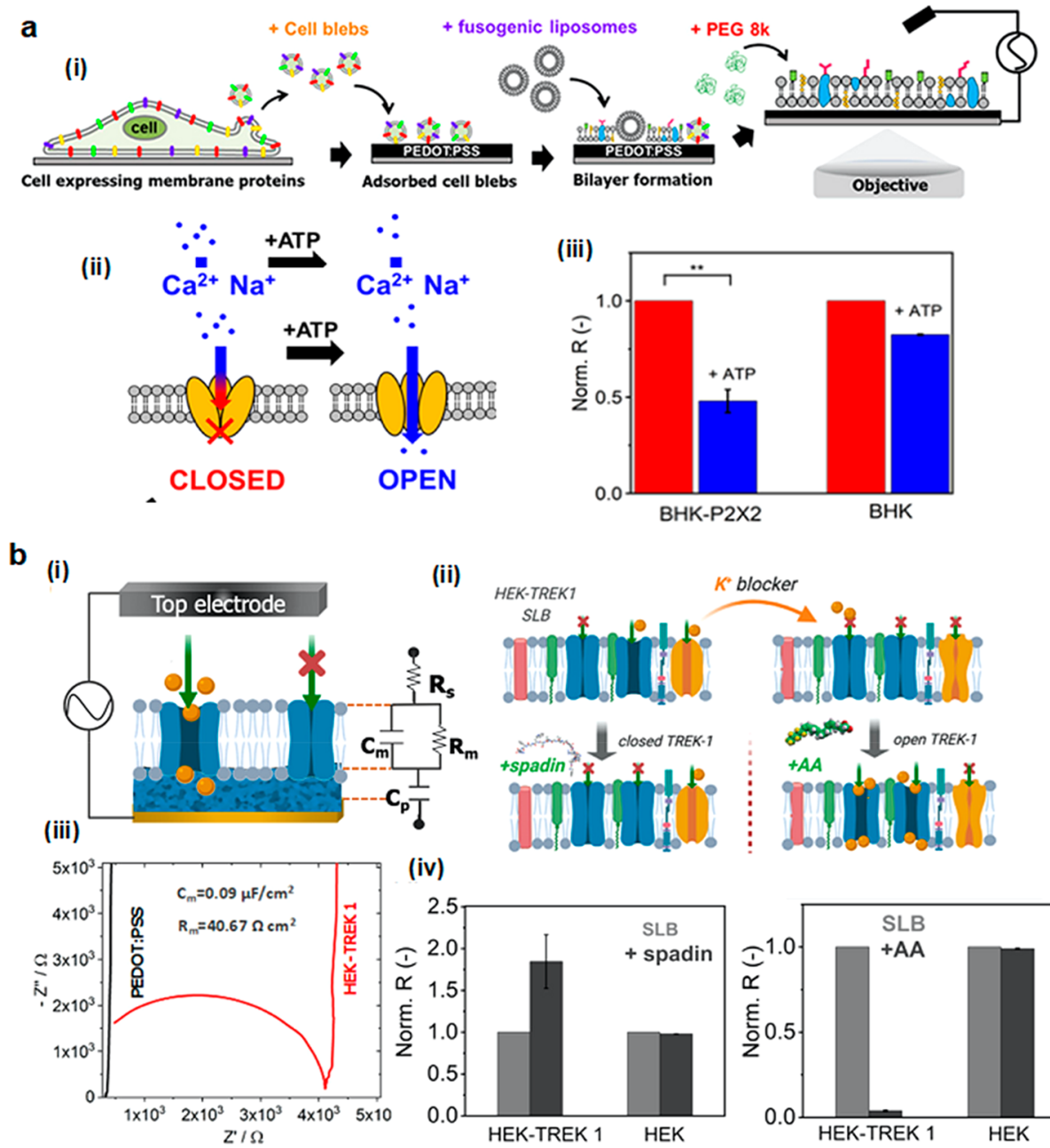

(iv)
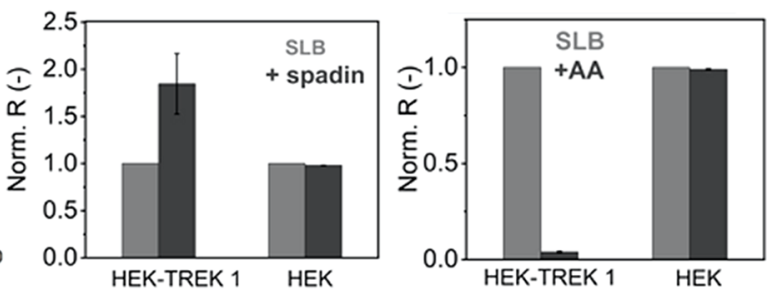

Figure 28. (a) Native baby hamster kidney cell SLB formation on PEDOT:PSS electrodes. (i) Schematic showing extracellular vesicle isolation, rupture, and self-assembly on PEDOT:PSS surfaces using fusogenic liposomes and soluble PEG(8000) for impedance measurements. (ii) Schematic showing a PX2X ATP-gated ion channel and (iii) calculated (normalized) membrane resistance of the P2X2 membrane before (red) and after (blue) ATP addition showing the decrease in the resistance of the membrane in response to ATP, and the corresponding values of the BHK membrane (lacking the P2X2 receptor) for comparison. Adapted with permission from ref 251. Copyright 2020 American Chemical Society. (b) Ion channel monitoring using PEDOT:PSS devices bearing human embryonic kidney SLBs with expressed TREK-1 channels. Schematics showing (i) the HEKTREK1 integrated PEDOT:PSS, where TREK-1 is shown as a blue TMP with orange cations passing through, and the equivalent circuit used for modeling the EIS data and (ii) the complex native membranes $\left(\mathrm{K}^{+}\right.$ion channels are depicted in yellow, with other TMPs in pink and green, while TREK-1 ion channels are shown in blue) showing the different drug additions and their effect on TREK-1 channel function. (iii) Nyquist plot of the EIS response of the HEK-TREK-1 membrane compared to the bare PEDOT:PSS electrode showing the characteristic formation of a semicircle after the SLB is formed and (iv) the extracted normalized resistance values of the membranes for spadin, which suppresses TREK-1 activity, thus increasing the resistance of the membranes, shown here normalized to the baseline SLB resistance, and AA that activates TREK-1 channels thus decreasing the resistance of the membranes shown here normalized to the baseline SLB resistance. Adapted with permission from ref 375 . Copyright 2020 American Chemical Society.

pids and cholesterol on PEDOT:PSS surfaces. The resulting models have been used to study membrane-drug interactions (Figure 26), ${ }^{376}$ as well as for the detection of binding events using lipids that bear proteins as biorecognition elements. ${ }^{377}$ As bacterial membrane mimics are more challenging to form on PEDOT:PSS films via vesicle fusion given the mutually negatively charged surfaces, SALB greatly facilitates this, obviating the need for surface treatment steps. Despite the universality of the method, a drawback of SALB lies in the use of organic solvents during SLB preparation, which renders protein incorporation challenging.

Another efficient strategy to promote SLB formation, as an alternative to surface treatment, is the de novo synthesis of materials that bear functional units that can favor SLB-material interactions, benefiting from the versatility in synthesis of CPs.
To this end, a newly synthesized conjugated polymer based on a naphthalene 1,4,5,8-tetracarboxylic diimide bithiophene backbone functionalized with lysine-based side chains was shown to facilitate the assembly of the zwitterionic lipid vesicles into an SLB, allowing the incorporation and electrical monitoring (via an OECT) of gramicidin pores (Figure 27). ${ }^{378}$

3.1.1. Toward Native Cell Membrane Models. Although SLBs represent a good platform to incorporate functional transmembrane proteins, they still pose certain drawbacks in terms of recapitulating the full complexity of the plasma membrane. ${ }^{251}$ Even SALB-based membranes have limitations in terms of the degree of complexity, hence rendering their use limited. Along these lines, the Daniel group has pioneered a method to construct membrane models that achieve the complexity of the cellular plasma membrane, allowing study of 
the structure and function of the embedded transmembrane proteins in their native environment. Using native membrane vesicles that self-assemble into SLBs on top of solid supports combines the advantages of both worlds. Such authentic membrane systems allow study of lipid-protein association in the presence of native membrane components, a much-needed intermediate between very simple bilayer platforms and whole cell readouts. ${ }^{251}$ Such SLB platforms containing the complete biological machinery of cell membranes have been recently integrated with organic electronic materials and devices by the Owens and Daniel teams. The use of cell membrane components to form the bilayer has proven to be compatible with the conducting polymer surface; hence good quality "native" SLBs can be successfully realized via vesicle fusion, exhibiting proper protein mobility and functionality. The use of electroactive substrates has opened up a variety of possibilities for on-chip cell membrane studies and for biosensing using electrical readouts without the need for bulky equipment. Up to now, the teams have demonstrated the use of the authentic cell membranes on-chip for studying ion channel activity in response to different drugs or other stimuli, both electrically and optically (given the optical transparency of the CP). This method can therefore readily serve as an alternative to patch clamp whole cell experiments. (Figure 28) 251,379 Very recently, this technology was implemented for fundamental understanding of membrane events governed by infection processes including virusmembrane interactions, ${ }^{380}$ as well as toxin binding. ${ }^{381}$

\subsection{D Cell Cultures}

3.2.1. Electrogenic Cells. One of the most common applications for in vitro bioelectronic systems is the growth, monitoring, and stimulation of electrogenic cells. These cell populations typically possess a series of molecular mechanisms that allow them to alter their local ionic environment, subsequently resulting in the generation of localized electric fields. These transient phenomena can be monitored or even induced through electrical devices in close proximity to the cell. Numerous advances have been made in the production of relevant technologies for improving the recording efficacy of signals from electrogenic cells. Two of the most commonly studied electrogenic cell types are neurons and cardiomyocytes due to their relevance to major body systems, the nervous system and the cardiovascular system, respectively. In this section, we focus on the growth and monitoring of electrogenic cells in 2D systems using organic bioelectronics.

3.2.1.1. Biology and Background for Electrogenic Cells. Electrogenic cells possess a membrane potential derived from differential maintenance of the concentrations of different ions in the interior of the cell versus the exterior. These concentration gradients originate from a series of selective ion channels, pumps, and exchangers that allow the cell to control its internal environment. ${ }^{382,383}$ When stimulated, these cells can depolarize through a mass influx of ions into the cell. This depolarization is mitigated by ionic pumping and exchange mechanisms that bring the cell back to its resting membrane potential. ${ }^{384}$ This process, termed an action potential (or sometimes spiking), is one of the primary bases for communication between electrogenic cells, occurring in both neurons and cardiomyocytes. $^{384,385}$ These signals occur over short time scales, a few milliseconds in neurons ${ }^{384}$ and hundreds of milliseconds in cardiomyocytes. $^{385}$ In neurons, this action potential can propagate along the neuron to a synapse, where, typically with a cohort of simultaneous signals from other neurons, ${ }^{382}$ it can cause a new neuron to fire another action potential either directly through an electrical synapse across a gap junction or, more commonly, through a chemical synapse by release of neurotransmitters. ${ }^{386}$ In cardiomyocytes, the action potential is typically transmitted directly through gap junctions between adjacent cells. ${ }^{385}$

As the depolarization of a neuron is synonymous with the attempted transmission of information, these cells typically subsist in network patterns, where neuronal firing can be tracked. In complex systems with many neuronal cells like the brain, slow waves of activity called local field potentials (LFPs), can also be observed. ${ }^{244,387}$ The ability to record an individual cell's action potential versus the LFP of a cellular cohort is largely dictated by the proximity of a recording device to that cell. ${ }^{388}$ Depolarization in cardiomyocytes also typically occurs in a pulsatile manner, concurrent with a physical contraction consistent with the beating of heart muscle. ${ }^{389}$ This depolarization also propagates in spatially resolved waves from a point of origin. $^{287,390-392}$ As such, the spread of information through networks of electrogenic cells has driven the production of spatially resolved instrumentation for recording of these signals.

Although the focus here is on organic bioelectronics for recording cell activity, other techniques are widely utilized in this field for monitoring electrogenic cells. Historically, the most common mechanism for recording electrogenic cellular activity is the patch-clamp technique. This method involves the placement of a glass pipet onto the surface of the cell membrane. A small suction is subsequently applied, resulting in a tight contact between the membrane and the pipet. Electrodes can be positioned inside the pipet to then apply a potential to the cell, and the following electrical activity of the cell can be monitored. ${ }^{393}$ Various iterations on this technique exist including the cell-attached configuration and the whole-cell configuration. The whole-cell configuration involves the rupture of the localized cell membrane within the interior of the circumferential contact of the pipet with the cell, thereby creating a continuity between the cell's interior and the interior of the pipet. ${ }^{393}$ This setup is convenient, as it allows application or measurement of potential across the membrane and is thus widely used. Another common method for monitoring electrogenic cell activity is calcium imaging, where a fluorescent marker for calcium is monitored. Typically, individual live cells are imaged under a fluorescent microscope, and the resultant change in the fluorescence of calcium-derived signals is recorded. ${ }^{394}$ Calcium is associated with various processes in electrogenic mechanisms ${ }^{394}$ and is often used broadly as a marker for firing activity. However, the pathways of action involving calcium flux in a cell can be specifically examined for the development of far more informative data. ${ }^{394}$

3.2.1.2. 2D Substrates for Electrogenic Cell Culture. Over the past two decades, numerous culture systems have been developed using organic electronic materials for electrogenic culture applications. Much of this work has focused on the efficacy of growing these cells and understanding their behavior within the confines of these material systems. Some of the earliest work in this area focused on the growth of a pheochromocytoma-derived cell line, PC-12, on a PPy substrate with applied electrical stimulation. ${ }^{152,161}$ This cell line is commonly used in neuronal studies, as these cells can be differentiated into neuron-like cells. The stimulation aspects of this study and others will be examined later in this review. Another early study examined neuron-related cell interactions with a PPy-coated electrode shank, designed for in vivo usage. ${ }^{395}$ 


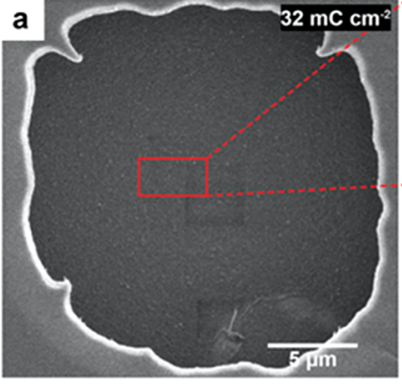

c I $100 \mu \mathrm{V}$

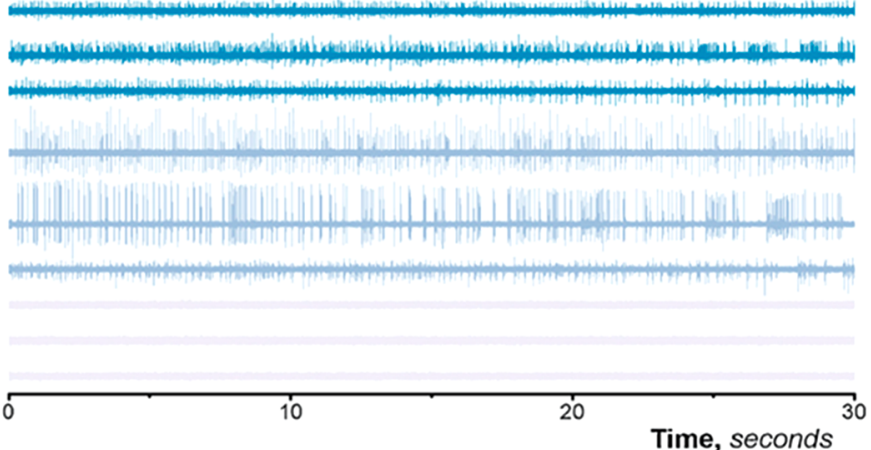

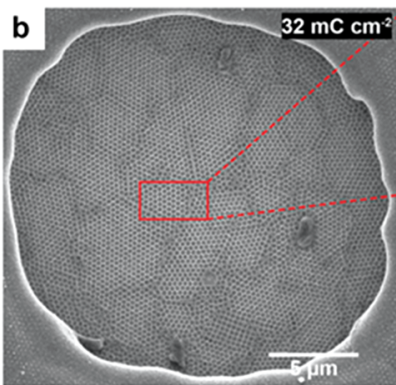
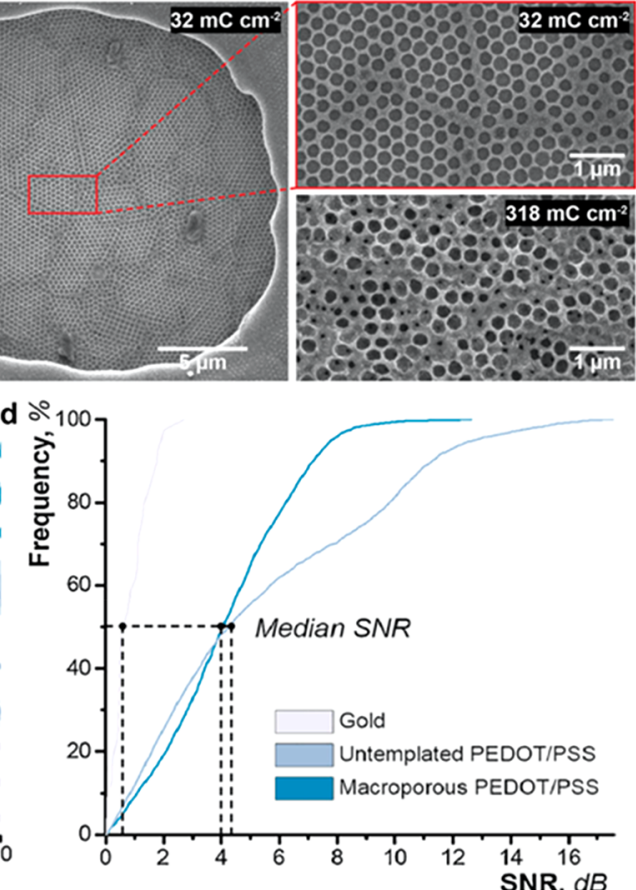

Figure 29. SEM images showing different PEDOT:PSS coatings on electrodes with resultant neural recordings. (a) Untemplated PEDOT:PSS-coated gold electrode with insets showing resultant deposition from different charge densities. (b) Macroporous PEDOT:PSS-coated gold electrodes showing resultant deposition from different charge densities. Macropores are generated using sacrificial templating process. (c) Recordings of spontaneous neural activity from primary hippocampal cultures. Time traces show resultant recordings from microporous PEDOT:PSS-coated gold electrodes (318 $\left.\mathrm{mC} \mathrm{cm}{ }^{-2}\right)$, untemplated PEDOT:PSS-coated gold electrodes $\left(318 \mathrm{mC} \mathrm{cm}^{-2}\right)$, and bare gold electrodes. (d) Signal-to-noise ratio versus cumulative frequency for coatings. Adapted with permission from ref 487. Copyright 2018 Elsevier.

In this section, we will discuss electrogenic cell cultures on electroactive substrates, highlighting a few key factors that have been considered by researchers in the past. We will then move toward the active monitoring of these cultures using various organic bioelectronic devices.

Many of the studies utilizing electroactive substrates have examined neural populations on PEDOT b a s ed $91,92,153,163,178,192,396-422$ P P y based, ${ }^{140,174,178,415,416,423-434}$ and PANI-based substrates, ${ }^{435-442}$ as well as other substrates including pentacene, melanin, P13, and P3HT. ${ }^{226,443-446}$ In many of these cases, researchers will often examine neurite outgrowth as an indicator of neural cell compatibility with a given substrate. A neurite is a protrusion from a neuronal cell, sometimes synonymous with an axonal or dendritic projection but generally referring to any observed cellular projection. Monitoring of this parameter is frequently paired with other commonly used biocompatibility metrics, such as viability, proliferation, cell spreading, and protein markers. For example, Abidian et al. examined the effects of different morphologies of PEDOT and PPy coatings on neuron spreading, finding that different polymeric morphologies altered the extent of neurite outgrowth. ${ }^{447}$ Another study showed that PEDOT can be electrochemically polymerized around SH-SY5Y cells, a cell line derived from a human neuroblastoma that can be differentiated toward neuron-like cells. ${ }^{448}$ Modulating the electrochemical properties of the substrate can also effect cell behavior. ${ }^{449}$ These studies show the compatibility of various organic bioelectronic culture environments and substrates with neural cultures.

Similarly, numerous studies have focused on the culture of cardiac cells on organic electronic substrates. Previous work has utilized PEDOT-based, ${ }^{450-453}$ PPy-based, ${ }^{454-457}$ and PANI- based substrates. ${ }^{458-460}$ Generally, these studies perform some biocompatibility assays to determine viability, proliferation rate, etc. Some works also monitor spontaneous beating rate through video analysis or calcium imaging. One study showed that geometrical considerations, that is, the shape of a scaffold (a tube versus a folding sheet), can also have an effect on beating frequency and contractile displacement. ${ }^{461}$ Wang et al. cultured primary rat cardiomyocytes on electrospun PLA/PANI sheets. Upon folding these sheets into different configurations, differences in beating frequency and maximum displacement were observed. ${ }^{461}$ These data indicate that cardiomyocytes can be manipulated using the culture system, but these differences must be examined as experimental iterations are introduced. One study also cultured cardiomyocytes on numerous organic semiconductors. ${ }^{462}$ This study compared viability, gauged using the MTT assay, a colorimetric assay that monitors metabolic activity, of cardiomyocytes on TIPS-pentacene, $\mathrm{DPP}(\mathrm{TBFu})_{2}$, P3HT, and PEDOT:PSS, as well as on culture plastic and glass, providing a comparative analysis of these substrates. The MTT assay showed slightly higher values for P3HT and slightly lower values for PEDOT:PSS versus cells on culture plastic, but all substrates performed well. ${ }^{462}$ Maráková et al. also generated films of PEDOT-graphene oxide (GO) and PPy-GO on either graphene paper or ITO for culture of neuronal and cardiac cells. ${ }^{463}$ Regardless, these studies show that different materials, geometries, etc., can all be used to manipulate fundamental biological phenomena, such as cell metabolism, in neural and cardiac populations.

Another large cohort of studies in this field have been conducted to determine the biocompatibility of organic electronic substrates prior to in vivo testing. Studies have cultured neuronal cells in vitro prior to in vivo experiments on 

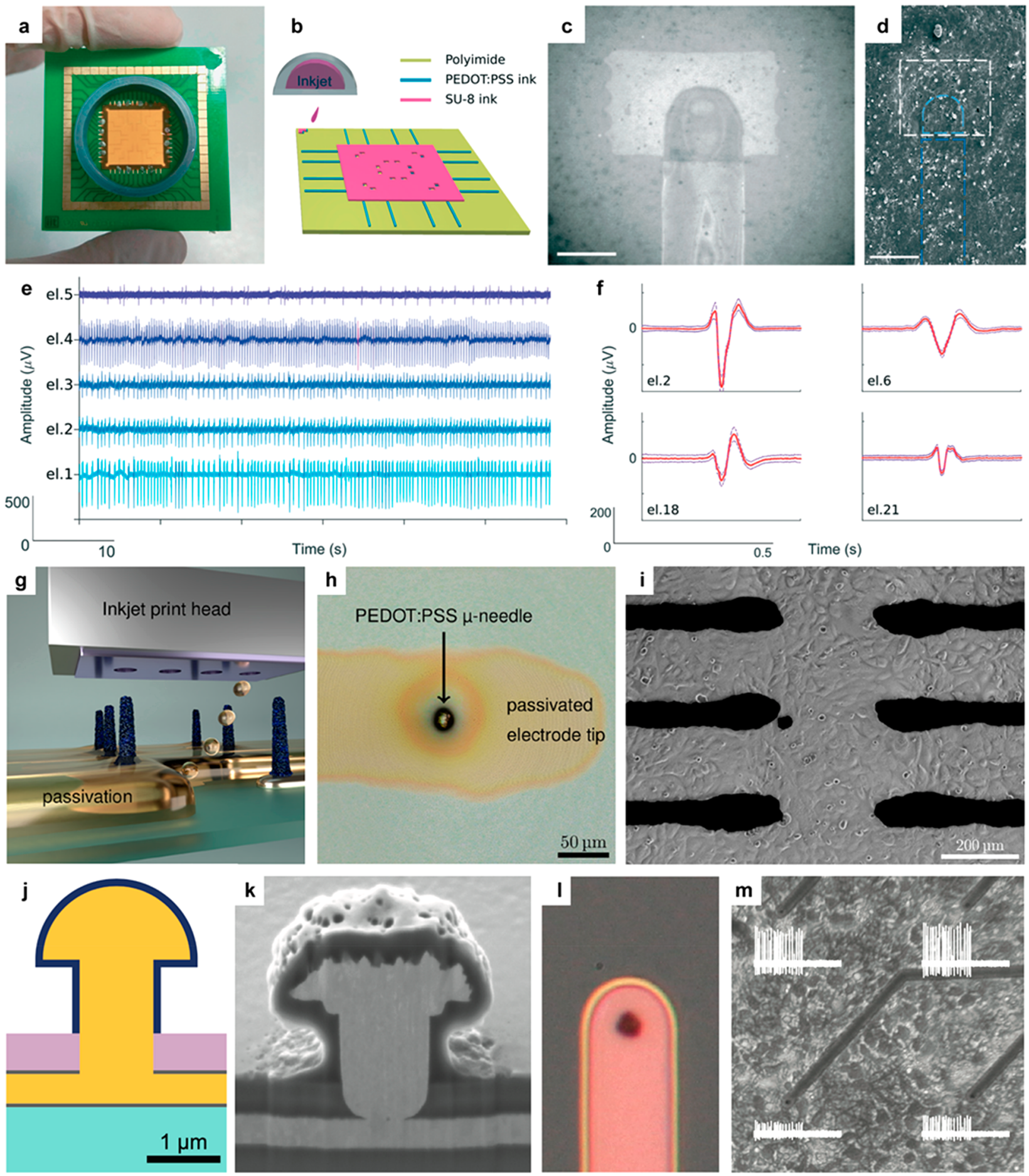

Figure 30. Images of different morphologies for MEAs. (a) Image of a MEA. (b) Schematic showing MEA structure. (c) Microscopic image of MEA recording site. Scale bar is $200 \mu \mathrm{m}$. (d) SEM image showing confluent layer of HL-1 cardiomyocytes with traces showing outline of MEA recording electrode. Scale bar is $200 \mu \mathrm{m}$. (e) Recording voltages from HL-1 cells on 5 different electrodes with (f) average waveform for each electrode. Adapted with permission from ref 258. Copyright 2019 Royal Society of Chemistry. (g) Schematic showing printing of microneedle electrode MEA. (h) Microscopic image of resultant electrode structure. (i) Image of HL-1 cardiomyocytes on microneedle MEA. Adapted with permission from ref 488. Copyright 2019 American Chemical Society. (j) Schematic of electrode structure with highlighted materials: PEDOT:PSS (black), gold (yellow), SiN $x$ (pink), Ti (gray), glass (blue). (k) SEM cross-section (focused ion beam) of PEDOT:PSS-coated electrode with (l) corresponding optical image. (m) Microscopic image of rat cortical cells grown on PEDOT:PSS-coated electrodes with voltage time traces (scale is $\sim 150 \mu \mathrm{V}$ by $4 \mathrm{~s}$ for larger traces) after 2 weeks of culture for each electrode. Adapted with permission from ref 489. Copyright 2020 Jones et al. published by Frontiers Media under Creative Commons Attribution 4.0 International License, https://creativecommons.org/licenses/by/4.0/.

PEDOT-based ${ }^{254,464-474}$ and PPy-based probes. ${ }^{47-477}$ These studies have primarily targeted the central nervous system. Other similar "preclinical" studies have been performed with cardiac cells as preliminary tests for cardiac patches, mostly using PANI-based ${ }^{478-480}$ substrates and poly(3-amino-4-methoxybenzoic acid) (PAMB)-based ${ }^{481,482}$ substrates. Kapnisi et al. 

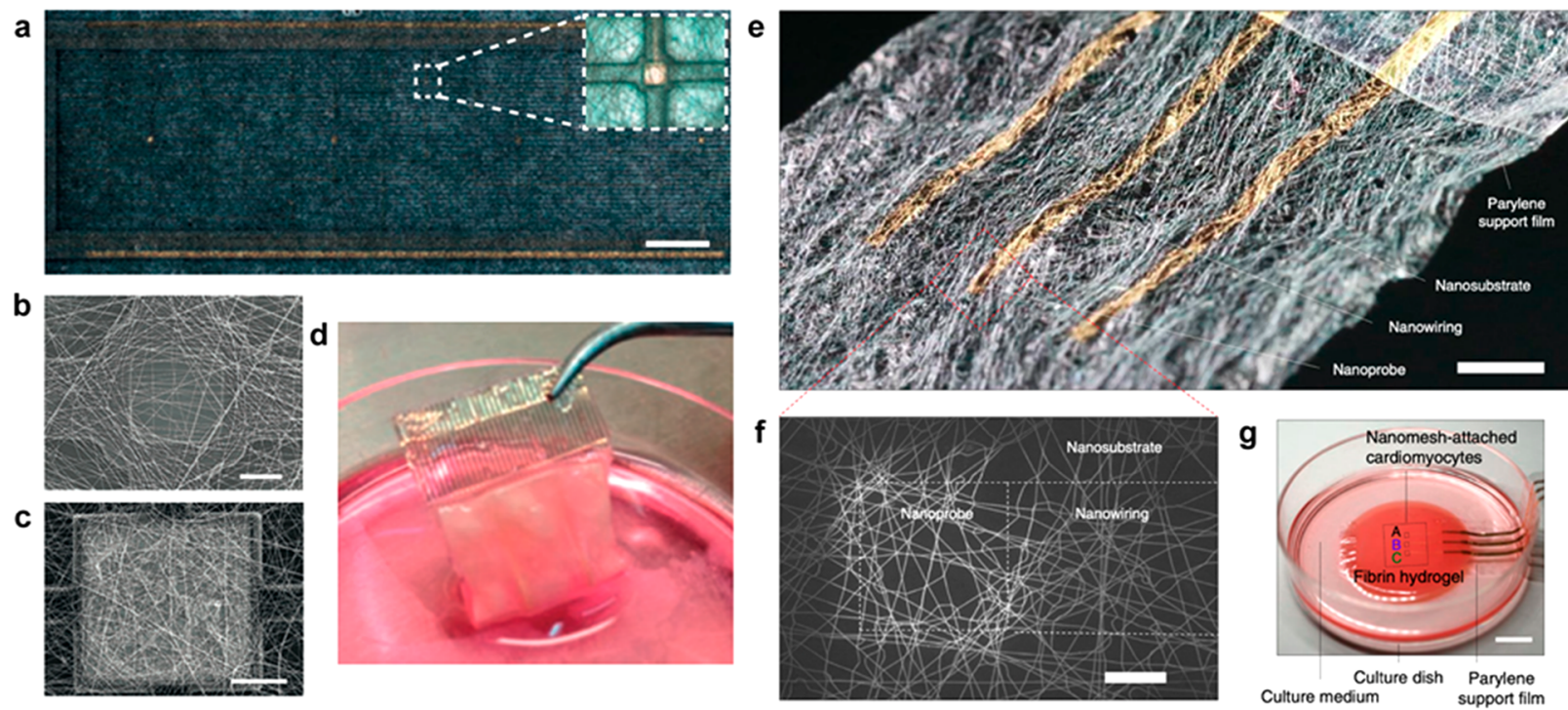

Figure 31. Images showing fibrous structures for recording information from electrogenic cells. (a) Image of fibers for cardiac patch with inset showing a recording electrode. Scale bar is $1.5 \mathrm{~mm}$. (b) SEM image of recording electrode. Scale bar is $20 \mu \mathrm{m}$. (c) Image of conducting polymer electrode, also on fibrous cardiac patch. Scale bar is $50 \mu \mathrm{m}$. (d) Image showing cardiac patch after 7 days culture with cardiac cells. Adapted with permission from ref 504. Copyright 2016 Springer Nature. (e) Image of fibrous structure with built-in electrodes (gold) for recording electrogenic signals. Scale bar is 2 $\mathrm{mm}$. (f) SEM image showing microstructure of mesh, allowing for connectivity between conductive regions. Scale bar is $200 \mu \mathrm{m}$. (g) Image of device in culture with cardiomyocytes for monitoring electrogenic signaling between cells. Scale bar is $1 \mathrm{~cm}$. Adapted with permission from ref 506 . Copyright 2018 Springer Nature.

also created a PANI-based cardiac patch using an auxetic structure. ${ }^{483}$ Auxetic materials and structures have a negative Poisson's ratio, meaning that when they are tensed, they expand in the orthogonal directions. These patches were found to maintain bulk conductivity in their auxetic structuring and conform to heart tissue during beating experiments, ${ }^{483}$ providing some insight into other mechanisms that can be employed during cardiac cell culture.

3.2.1.3. Organic Bioelectronic Devices for Monitoring Electrogenic Cells in 2D. While various substrates have been produced for the culture of electrogenic cell populations, numerous have also been developed for in vitro culture and monitoring. Here, we will discuss different organic electronic technologies that have been applied primarily for the measurement of action potentials and spiking activity from neuronal and cardiac cultures. Given that electrogenic cells tend to perform functions in spatially resolved manners, as mentioned above, the most common device for monitoring these cultures is the electrode and more specifically the MEA. As electrogenic cells fire action potentials, the generated electric field can be observed through locally placed electrodes. This methodology has been widely used with neuronal cultures, as MEA devices are also frequently applied for cortical monitoring in vivo. ${ }^{484}$ MEA devices for these applications are typically prepared by coating a metal or indium tin oxide (ITO) electrode with an electroactive material, usually a conductive polymer. This coating is known to enhance recording efficacy and improve signal-to-noise ratio (SNR). ${ }^{62,388,485}$ However, researchers have also generated MEAs for in vitro recordings entirely out of organic electroactive materials. ${ }^{258,486}$ Coating morphology can significantly affect recordings and SNR. Aqrawe et al. electrochemically polymerized PEDOT:PSS at different charge densities onto an array of gold electrodes in either an untemplated (Figure 29a) or a templated manner, using sacrificial poly(styrene) beads to generate pores in the PEDOT:PSS coating (Figure 29b). ${ }^{487}$ To test the efficacy of these different coatings, primary hippocampal neurons were cultured on the MEAs, and recordings of spontaneous firing were taken after 16 days of culture from uncoated, templated, and untemplated gold electrodes. Representative time traces indicate that the SNR was highest for the templated electrodes, followed by the untemplated electrodes, and, finally, the uncoated electrodes (Figure $29 \mathrm{~b}, \mathrm{c}){ }^{487}$ This study also utilized a stimulating regimen for these neuronal cultures, which will be discussed later in the review.

Numerous other MEA formats have been produced, including recessed PEDOT:PSS electrodes (Figure 30a-f), ${ }^{258}$ PEDOT:PSS microneedle electrodes (Figure $30 \mathrm{~g}-\mathrm{i}$ ), ${ }^{488}$ and mushroom-shaped electrodes (Figure $30 \mathrm{j}-\mathrm{m}$ ), ${ }^{489}$ among others. All of these electrode formats appear to show similar cellular compatibilities, at least from a visual standpoint, regardless of geometry (Figure 30d,i,m). ${ }^{258,488,489}$ Recordings of neuronal cells can even be collected through arrays of conductive hydrogel electrodes. ${ }^{490}$ However, comparison of cellular compatibility for different geometries is difficult, given the wide range and variance among culture conditions and cell types. With proper material selection and preparation, MEAs can also be constructed to be transparent, ${ }^{255,256,491}$ which is convenient for monitoring cells visually during recordings, especially given that proximity of cells to a particular recording electrode is of great relevance to the resultant data quality. Of note, as MEAs essentially consist of various individual recording units, the recordings can and will vary for each individual MEA (Figure 30e,f). ${ }^{258}$ However, with this in mind, beating synchronicity can be examined in cardiac cultures using MEAs. Recordings in these systems can also be qualified for both neuronal and cardiac cultures through examination of resultant signals before and after application of drugs that affect firing. For example, bicuculline and tetrodotoxin can be applied to neuronal cultures to induce increased or decreased firing rates, respectively. ${ }^{492}$ Similarly, isoproterenol, ${ }^{493}$ norepinephr- 
ine, ${ }^{304}$ and other drugs can be applied to modulate beating frequency in cardiac cultures.

Many other studies have performed MEA recordings using primarily PEDOT-based setups on neuronal ${ }^{492,494-497}$ and cardiac cultures, ${ }^{498,499}$ as well as a few other electroactive materials. ${ }^{500,501}$ Other interesting systems include a PEDOTbased electrode system developed by Smith et al. with an ionpermeable, patterned Nafion top layer to allow the application of different topologies on top of the MEA surface. Both neuronal and cardiac cultures were applied to this device for successful recordings of each, ${ }^{502}$ indicating that different topologies and layers can be applied on top of MEA structures while maintaining recording efficacy. Heizmann et al. also produced a PEDOT:PSS-based electrode array designed to release dyes that can penetrate into SH-SY5Y cells upon oxidation of the PEDOT:PSS electrodes. ${ }^{503}$ While this study does not represent an active recording of a neuronal or cardiac population, it does show the potential for tracking cells that have been recorded, which could be very useful if combined with some of the above technologies. Finally, a few devices have been manufactured that show great potential for application within the field of organic bioelectronics. Feiner et al. developed a cardiac patch by fabricating a series of gold and PPy electrodes on a mesh substrate (Figure $31 \mathrm{a}-\mathrm{d}$ ). ${ }^{504}$ This substrate was then coated in an electrospun scaffold to promote cell adhesion (Figure 31a). The gold electrodes (Figure 31b) allow for cardiomyocyte recording and stimulation, while the PPy electrodes (Figure 31c) provide a drug release mechanism within the device. The overall device construction also allows for a free-standing structure (Figure 31d). ${ }^{504}$ An updated version of this device was constructed by directly patterning the electroactive materials onto the mesh. ${ }^{505}$ Lee et al. constructed a similar type of device (Figure $31 \mathrm{e}-\mathrm{g}$ ) through the deposition of gold onto a nonelectroactive polymeric substrate (Figure 31e,f), showing that cardiomyocyte activity can be recorded using this format (Figure $31 \mathrm{~g}) .{ }^{506}$ These device layouts are generally compatible with many of the different techniques utilized for recordings that have been mentioned in this section and provide considerable customizability for future in vitro culture systems.

Transistors have also been developed for monitoring electrogenic cell populations but to a much lesser extent because of complex circuitry. Some of the earlier instances of transistors designed for recording electrogenic cells utilized OFETs. ${ }^{507,508}$ These particular arrays were designed to have a channel that extends spatially beyond the source and drain, allowing for sensing of cells away from these components. These devices have been applied to record both cardiomyocytes ${ }^{508,509}$ and neurons. ${ }^{510}$ Kyndiah et al. constructed an EGOFET array for recording cardiomyocyte action potentials by examining the source-drain current, as mentioned above. Recordings from these devices were qualified using a series of drugs to modulate beating frequencies. ${ }^{304}$ Multiple device arrays have been constructed for monitoring cardiac cultures using OECTs. ${ }^{58,287,390-392,511}$ Liang et al. fabricated an array of PEDOT:PSS-based OECTs with interdigitated source and drain electrodes (Figure 32a). ${ }^{287,392}$ Monitoring the source-drain voltage in the presence of HL-1 cardiomyocyte-like cells enabled recording of cell activity (Figure $32 \mathrm{~b}$ ). By examining the activity on subsequent OECTs, Liang et al. were able to show the propagation of action potentials across this culture system (Figure 32c). ${ }^{287}$ Other OECT devices have been developed for monitoring electrogenic cell activity in vivo, ${ }^{512}$ but these devices have not yet been applied in vitro.

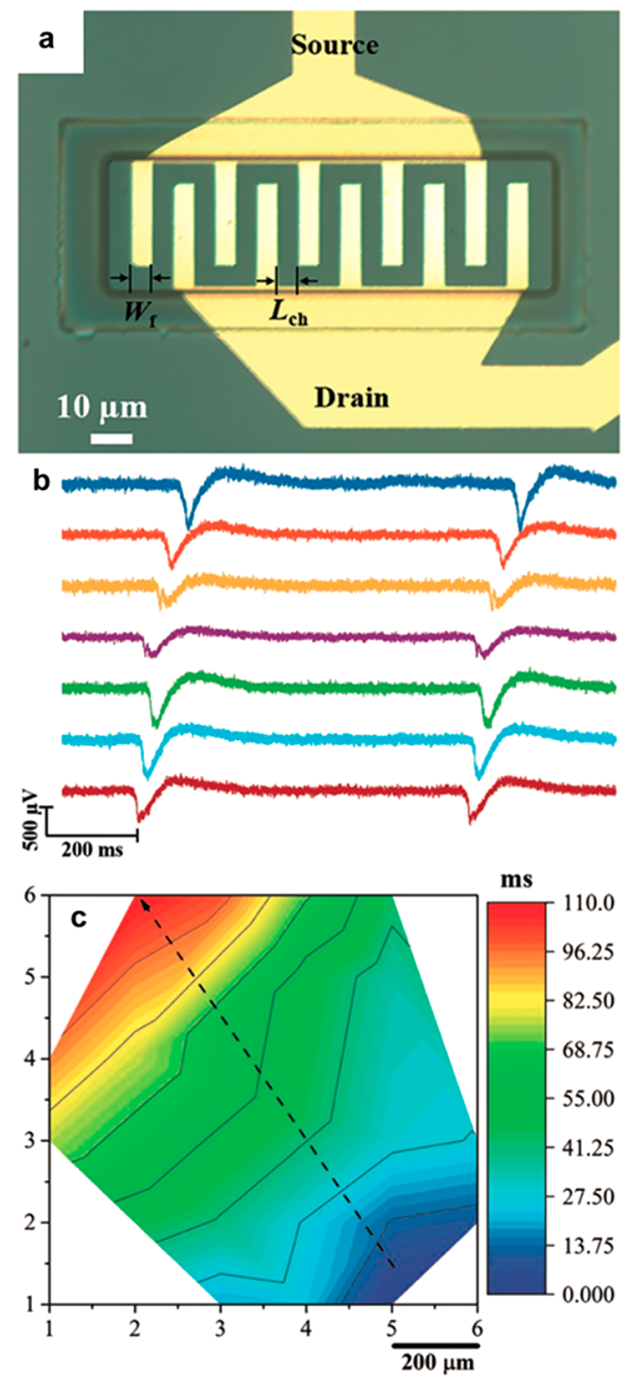

Figure 32. Image of OECT for recording cardiomyocyte behavior. (a) Microscopic image of OECT recording site. (b) Simultaneous recording of drain-source voltage in the presence of a confluent layer of HL-1 cardiomyocytes for 7 electrodes. (c) Contour plot of wave propagation across device as recording by OECT with HL-1 cells. Adapted with permission from ref 287. Copyright 2019 Wiley-VCH Verlag GmbH \& Co. KGaA, Weinheim.

Outside of transistors and electrodes, some studies have focused on the development of other recording modalities. Habib et al. fabricated an array of PEDOT:PSS-based nanoantennae, showing recording efficacy for cardiomyocytes. ${ }^{513}$ Santoro et al. generated a PEDOT:PSS-based substrate that showed changes in impedance associated with cardiomyocytes cultured on different topographies. ${ }^{514}$ Kielar et al. coupled the concept of calcium imaging with an organic photodetector to record activity from cortical neurons. ${ }^{515}$ Finally, the rapidly developing field of artificial synapses has also begun to show integration between these devices and live neurons. Desbief et al. produced an EGOFET synapstor, onto which they seeded neurons. ${ }^{303}$ Juzekaeva et al. developed a memristive device that they interfaced with neurons, showing that the neurons behave differently when interacting with the device. ${ }^{516}$ Neuromorphic devices, such as the synapstor and memristor, are not covered extensively in this review, but it should be noted that these devices will become increasingly pervasive, particularly as recent works show improved interaction between cell and device. ${ }^{517}$ 
a

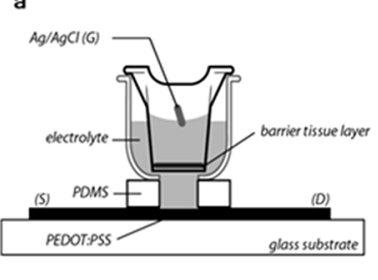

CROSSSECTION

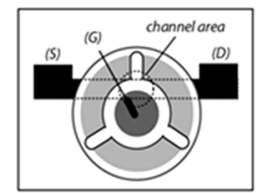

TOP

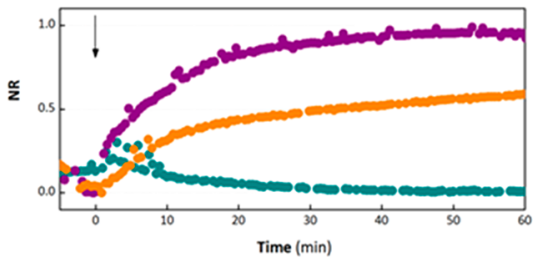

b

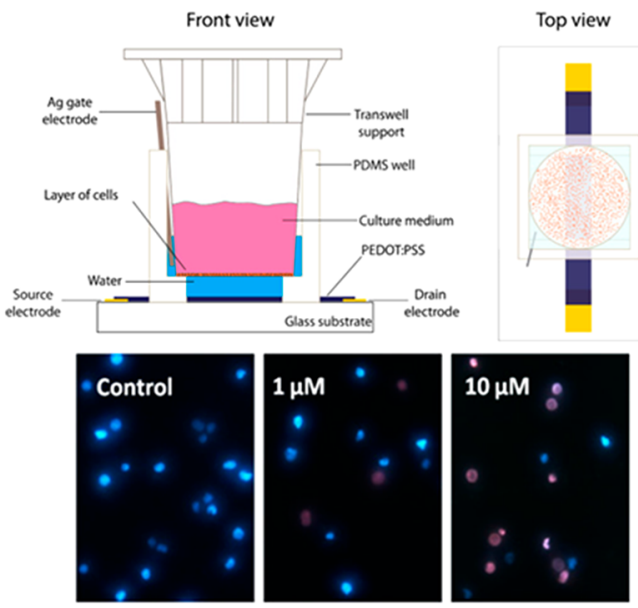

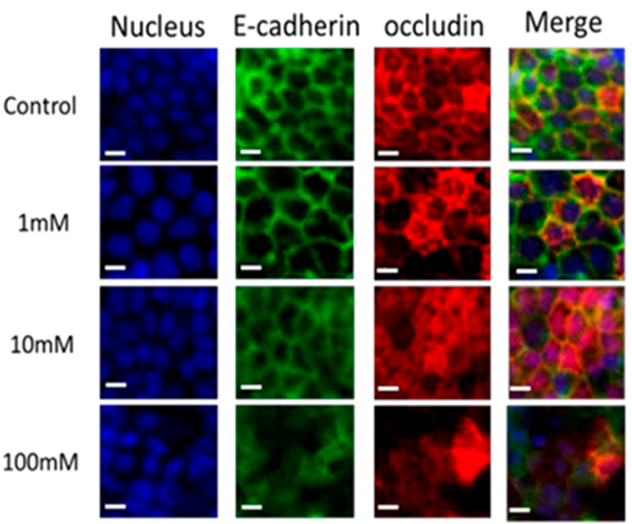

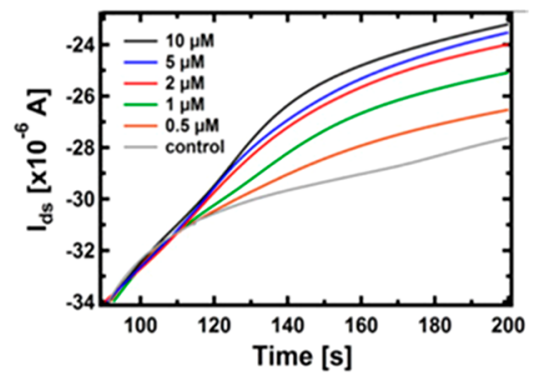

Figure 33. (a) Illustration of organic electrochemical transistor (OECT) barrier tissue sensor (top left). Immunofluorescence images stained for tight junction proteins (E-cadherin (green) and occludin (red), counterstained for nuclei (blue)) in the apical domain of the samples upon exposure to various concentrations of EGTA for $2 \mathrm{~h}$ (scale bars $10 \mu \mathrm{m}$; right panel). In situ monitoring of OECT normalized response (NR) upon addition of $1 \mathrm{mM}$ (dark cyan), $10 \mathrm{mM}$ (orange), and $100 \mathrm{mM}$ (violet) EGTA. EGTA is added at time $=0$, as indicated by the arrow; Reproduced from ref 533. Copyright 2013 MDPI under Creative Common Attribution-NonCommercial-ShareAlike 3.0 Unported (CC BY-NC-SA 3.0), https://creativecommons.org/ licenses/by-nc-sa/3.0/. (b) Experimental setup of the Twell-OECT device used as a sensor for in vitro monitoring of cellular dynamics (top left). Representative images of a live/dead assay on human A549 lung cells growing on the device after a $72 \mathrm{~h}$ exposure to increasing doses of doxorubicin (bottom left) and corresponding output characteristics of the OECT (kinetics of $I_{\mathrm{ds}}$ current) for the different concentrations of doxorubicin (right). Reproduced with permissions from ref 534. Copyright 2015 Elsevier.

These studies show that a broad range of techniques and technologies can still be developed and applied in vitro for neuronal and cardiac monitoring.

3.2.2. Nonelectrogenic Cells. 3.2.2.1. Biology and Background of Nonelectrogenic Cells. In the case of nonelectroactive cells, electrical measurements can provide information about cell viability, coverage, growth, and differentiation, as well as cell layer integrity for barrier-tissue forming nonelectrically active cells. In the body, such tissues act as physical barriers against the external environment (e.g., skin, lung, intestine) or as boundaries of biological compartments (e.g., blood-brain barrier). Barrier tissues are formed by one or more epithelial or endothelial cell layers, where cells are joined to each other by a complex network of intercellular junctions with tissue-dependent morphological and functional properties, which, along with the cells, determine the functionality of the barrier. Tight junctions, in particular, form strands that circumferentially band adjacent cells at the apical domain of the intercellular space, preventing molecules between the apical and lateral membrane domain from intermixing (i.e., fence function), acting as a gasket that regulates free diffusion of most solutes and transport of ions along the lateral intercellular space (i.e., gate function). ${ }^{518-521}$ Analysis of the functionality and integrity of tight junctions can be indicative of the barrier tissue state, under both physiological and pathological conditions or toxic events. To this end, many barrier tissue in vitro models are available, which, typically, are based on cell monolayers grown on filter membranes suspended in culture medium (i.e., Transwell-based culture formats), mimicking the apical-basal polarization of native tissues. ${ }^{345}$ Electrical methods can be used within these cell culture setups for rapidly monitoring cells in vitro and for quantitatively characterizing the function and integrity of barrier tissues. ${ }^{38,349}$ A well-established tool, widely used for this purpose, is TEER, which provides a means to quantify the ion flux across the barrier, tightly regulated by the gate function of tight junctions. ${ }^{518}$ One of the most common techniques for measuring TEER is the use of chopstick electrodes, placed on either side of the epithelial/endothelial monolayers. However, this method allows only for an approximate estimation of the ohmic resistance of the barrier 
a

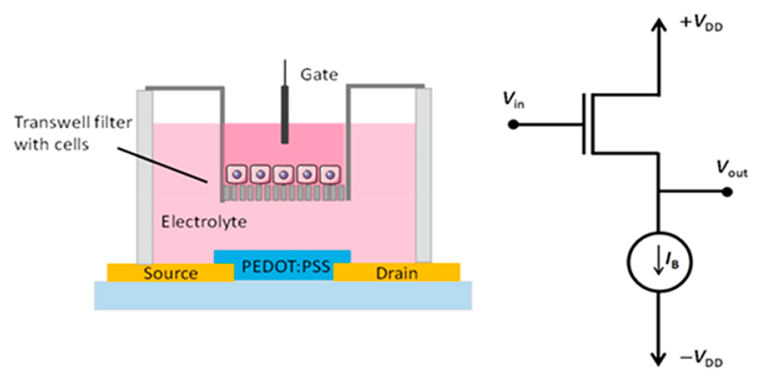

b

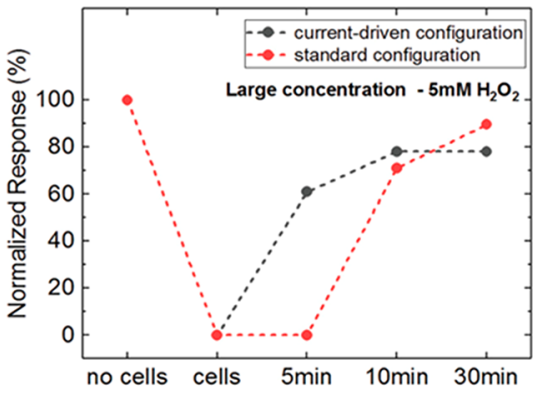

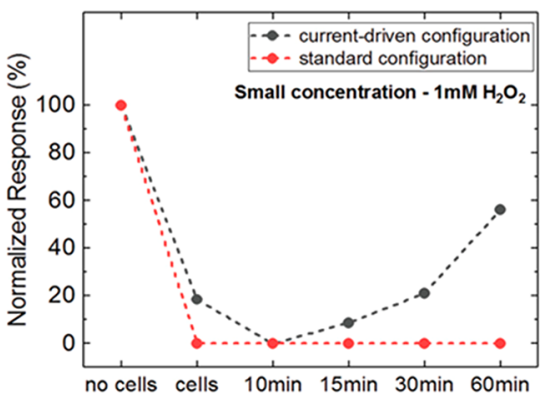

Figure 34. (a) Schematic of an OECT device used as a sensor of the barrier integrity of cells growing on the integrated Transwell filter and the equivalent circuit of the current-driven configuration of the device. (b, c) Graphs illustrating the normalized performance of the OECT as a cell barrier integrity sensor for different concentrations of $\mathrm{H}_{2} \mathrm{O}_{2}$, both in current-driven and in the standard configuration. $\mathrm{Ag} / \mathrm{AgCl}$ gate electrode and EMEM cell culture medium as an electrolyte were used. Reproduced with permission from ref 535. Copyright 2019 The Authors, published by WILEY-VCH under Creative Commons Attribution 4.0 International (CC BY 4.0), https://creativecommons.org/licenses/by/4.0/.

tissues, as it relies on single-frequency measurements, limiting the range of biological information that could be retrieved. Impedance spectroscopy can be used instead, providing a more accurate insight into various biological processes, including cell proliferation and differentiation, alongside barrier integrity of certain epithelial/endothelial tissues. In contrast to singlefrequency TEER measurements, EIS operates over a wide range of frequencies by application of appropriate modeling (See section 2.4). ${ }^{48}$ Changes in cell layer capacitance can be associated with the surface area and composition of the layer and thus with the morphology of the barrier tissue (i.e., apicalbasal polarization, presence of tissue specific structures such as microvilli). ${ }^{349,518}$ The sensitivity of this method to both cell density and morphology favored its application in a variety of assays, such as cell adhesion and proliferation, cell differentiation, wound healing, and barrier tissue integrity, among others. ${ }^{48,522-524}$ This technique has also been integrated with Transwell-based cell culture formats to allow for drug and nutrient transport studies (e.g., various electrodes and commercially available devices, such as CellZScope, Nanoanalytics $\mathrm{GmBH}){ }^{525-527}$ However, in most cases, these devices use rigid, high impedance electrodes (e.g., gold, silicon oxide). Here, we discuss the integration of organic bioelectronic devices in cell-based assays, as well as the added benefits they bring for cell monitoring.

3.2.2.2. Organic Bioelectronic Devices for Monitoring Nonelectrogenic Cells in 2D. While a number of studies laid the foundation for using organic electrical transducers as biosensors (section 4), ${ }^{528}$ it was only in 2010 that the first device was used to monitor the activity of nonelectrogenic cells (both barrier-forming and non-barrier-forming cell lines). In this work, Lin and collaborators ${ }^{529}$ employed a PEDOT:PSS-based OECT to electrically evaluate the integrity of cell layers formed by human esophageal squamous epithelial cancer cells (i.e.,
KYSE30) and fibroblasts (i.e., HFF1 cells) on top of the transistor channel. By evaluating the output characteristics of the OECTs upon disruption of either cell layer, the authors showed that the modulation of the transistor properties, as a result of the cell-semiconductor interactions, are cell type or tissue dependent. $^{529}$

A significant body of work on the suitability of bioelectronic devices for interfacing with biological systems has followed this application, and over the past decade a breadth of research projects have benefited from the continuous and noninvasive cell monitoring capabilities of such tools. ${ }^{264,530}$ Of particular importance is the integration of OECTs with barrier tissues (e.g., intestinal, kidney, blood-brain barrier), for sensing their integrity, known to dramatically alter after assault. As ion flow is tightly regulated in these biological models, integration of OECTs with barrier forming tissues is particularly favored due to the high sensitivity and sufficient temporal resolution of these devices for monitoring biological ion flux. ${ }^{531}$ This was first shown by Jimison et al. ${ }^{532}$ who modified the conventional OECT configuration by integrating filter membrane supports (Figure 33) commonly used for building barrier tissue models in vitro. Such filter membranes mimic the compartmentalized architecture of barrier tissues, providing cells with the necessary cues to form polarized monolayers and thus facilitate studies looking at nutrient/drug transport mechanisms and toxicity. This work demonstrated the compatibility of this new, nonplanar OECT configuration with existing protocols and assays for characterizing the integrity of an intestinal barrier model, grown from human intestinal epithelial cells (i.e., Caco-2 cells). The presence of the barrier tissue modifies the ionic flux, acting as an additional circuit element and inducing a slower response of the device (increase of response time, $\tau$, and drop of current, $I_{\mathrm{D}}$ ), while addition of barrier-disrupting compounds improved the response of the device. The OECT device was 

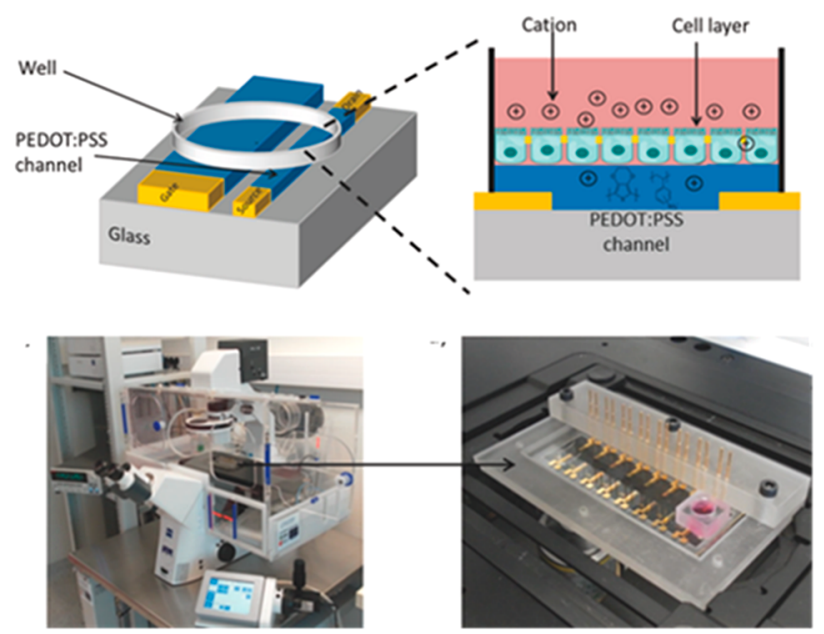

b

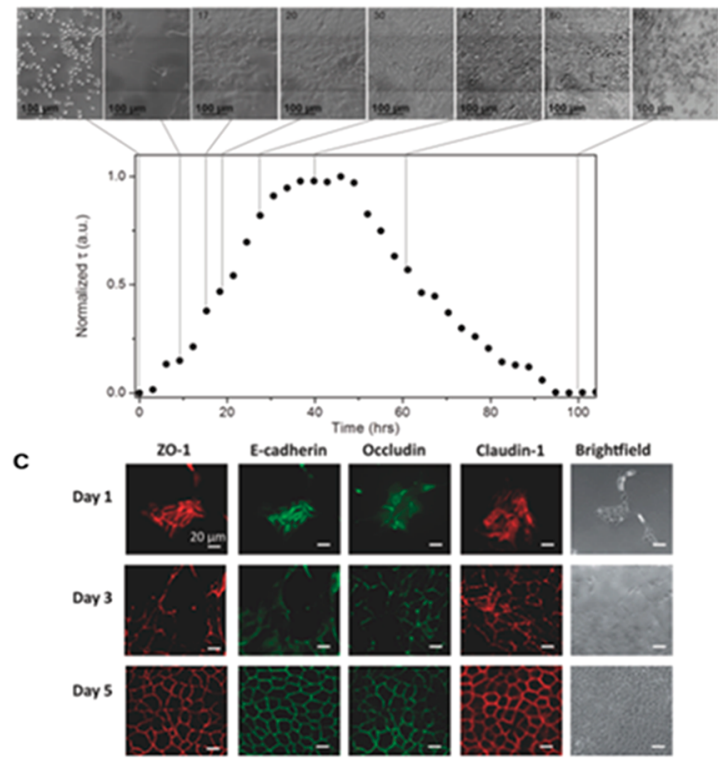

Figure 35. (a) Schematic illustration and pictures of a planar OECT for combined optical and electronic monitoring of the health status of live cells in vitro. (b) Electrical and optical characterization of MDCK-I cells growing on the PEDOT:PSS film of the planar OECT, (c) Micro-optical images of MDCK-I on top of the channel area (darker horizontal lines in the top panel images) and the corresponding tau values obtained at different time points (middle right panel). Immunofluorescence staining images of subconfluent MDCK-I monolayers revealing the presence and colocalization of epithelial junction proteins (ZO-1, E-cadherin, occludin, and claudin-1) over the 5-day cell culture experiment. Reproduced with permissions from ref 541. Copyright 2014 The Authors, published by WILEY-VCH under Creative Commons Attribution-NonCommercial-NoDerivatives 4.0 International (CC BY-NC-ND 4.0), https://creativecommons.org/licenses/by-nc-nd/4.0/.

shown to outperform state-of-the-art assays (e.g., permeability assays, immunofluorescence staining), acting as a highly sensitive sensor of the epithelial cell integrity, thanks to the inherent amplification properties of the transistor that allow for detection of early, minute changes in the ionic flux. This was further exemplified in a subsequent study by Tria et al., who demonstrated that the OECT can detect breaches of an integrated Caco-2-derived intestinal barrier upon exposure to EGTA (ethylene glycol-bis( $\beta$-aminoethyl ether)- $N, N, N^{\prime}, N^{\prime}$ tetraacetic acid) with the same sensitivity as commercially available EIS techniques, but with significantly increased temporal resolution. EGTA is a calcium chelator, commonly used to disrupt the tight junctions, which rely on calcium to form. Figure 33a illustrates the dose dependent responses to addition of EGTA: increasing concentrations of EGTA induce delocalization of the junctional proteins from the cell periphery and thus dysregulation of the paracellular ion flux, reflected in the improved normalized response of the OECT sensor upon disruption of the barrier tissue. ${ }^{533}$ Inspired by this work, another team of researchers developed an OECT combined with a Transwell support, the "Twell-OECT", and demonstrated its application as a sensitive diagnostic tool for direct monitoring of cellular dynamics and drug effects, yielding responses comparable to standard methods, but in a direct and dynamic manner. The configuration of the device (placement of the gate electrode between the PEDOT:PSS channel and the porous membrane; Figure 33b) led to the efficient detection of signals related to cell death mechanisms (e.g., apoptosis, necrosis), as application of voltage at the gate pushes to the OECT channel only ions that have crossed the layer and hence modulation of the drain current depends entirely on cell viability. ${ }^{534}$

In an attempt to further enhance the sensitivity and temporal resolution of OECTs for monitoring barrier integrity of cell monolayers growing on filter membranes suspended above the channel, Lingstedt et al. ${ }^{535}$ recently proposed the operation of the transistor in the current-driven configuration (Figure 34a). While in other approaches, described earlier, the OECT transient response to demonstrate barrier integrity measurement (time domain $)^{533}$ or cell coverage and differentiation (frequency domain $)^{60}$ is used, in the current-driven configuration the transfer curve of $V_{\text {out }}-V_{\text {in }}$ is used as a figure of merit. To test whether the new configuration is more sensitive in detecting changes in the cell barrier integrity, they compared OECT transient response measurements with current-driven operation mode upon disruption of intestinal barriers with different concentrations of $\mathrm{H}_{2} \mathrm{O}_{2}$. As demonstrated by the normalized response graphs in Figure 34c, both methods can directly detect the effects of peroxide on the cell barrier. In the case of high concentration, the current-driven configuration of the OECT can detect rupture of cell layer and cell opening with higher time resolution, while the finding in the low peroxide concentration exposure indicates the improved sensitivity of the method, which appears to be able to monitor in real-time the specific effects of peroxide on the cell barrier (i.e., disruption of tight junction, followed by cell opening). This increased sensitivity is explained by the differences in the operation mode and the specific device parameters, highlighting the fact that not only do OECTs have the ability to dynamically monitor barrier integrity outperforming conventional methods, but the device operation mode can be tuned to achieve different levels of sensitivity and resolution, tailored to each application.

The characteristics of the aforementioned nonplanar configuration of OECTs have been exploited in a variety of applications, including not only monitoring of the global cell status of both barrier forming ${ }^{532-534,536}$ and non-barrierforming cells, ${ }^{350,537}$ but also toxicology and drug transport, which have been favored by the transitioning toward more highthroughput platforms for long-term experiments. ${ }^{275,538}$ Although such OECT platforms enable optical access to the channel, ${ }^{349,539}$ simultaneous cell imaging is challenging, if not 
a

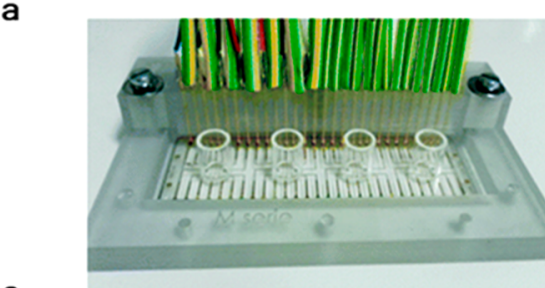

b

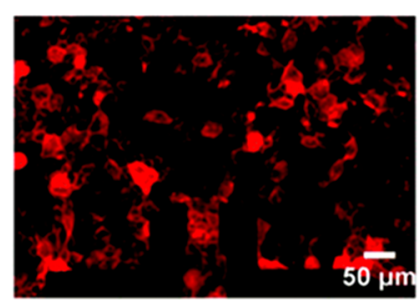

COVERAGE

adherent, non-barrier forming cells

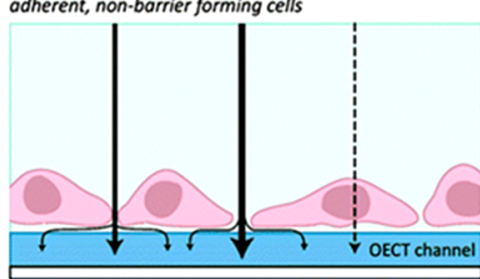

BARRIER PROPERTIES

barrier forming cells

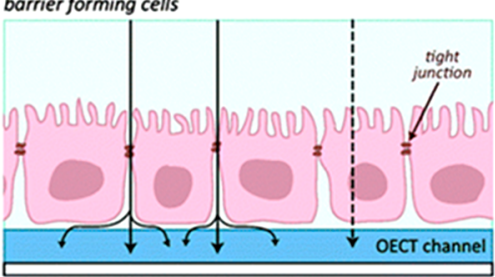

d

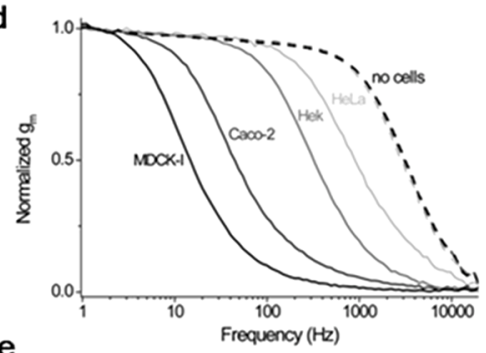

e

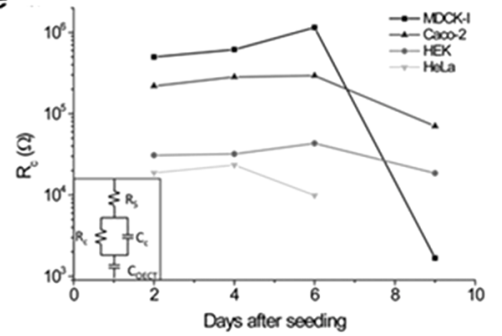

Figure 36. (a) Picture of an OECT platform for monitoring adherent cells, which consists of 24 OECTs divided between 4 glass wells. (b) Optical fluorescence image of RRF-transfected MDCK II cells directly seeded on the OECT channel. (c) Schematic illustrating the cell coverage associated with either high ion flow through non-barrier-forming cell layers (left) or low ion flow through barrier forming cell layers (right). Full vertical arrows indicate paracellular pathway, dashed vertical arrows indicate the transcellular pathway, and curved arrows indicate subcellular pathways; Electrical characterization of HeLa, HEK, Caco-2, and MDCK I cells using the OECT. (d) Normalized transconductance $\left(g_{\mathrm{m}}\right)$ at day 6 (full lines) of the respective cell line culture, compared with no cells (dashed lines). (e) Evolution of the cell resistance $\left(R_{\mathrm{c}}\right)$ of each type of cell culture, extracted from a simple fit using the equivalent circuit in the inset. Reproduced with permission from ref 350. Copyright 2015 Royal Society of Chemistry.

impossible since cells are grown on the filter support above the OECT channel. While this is of paramount importance for epithelial and endothelial barrier modeling, this configuration is incompatible with high-resolution live cell monitoring, rather forcing for end-point optical assays, after removing the filter membrane from the device. These limitations can be circumvented by the integration of cells directly on top of the OECT channel. Furthermore, this endows higher sensitivity for sensing integrity of biological barriers even in cases where the resistance is not very high. ${ }^{350}$ While Yao et al. ${ }^{540}$ were the first to demonstrate direct coupling of cell ion current with OECT channel current, Ramuz et al., ${ }^{541}$ taking advantage of the optical transparency of PEDOT:PSS OECT, proposed a planar OECT configuration for simultaneous optical and electrical monitoring of epithelial cells (Figure 35a). Madin-Darby canine kidney (MDCK)-I cells were seeded on the OECT channel and, by use of the response time, $\tau$, as a figure of merit of electrical characterization combined with high-resolution time-lapse images, allowed correlation of electrical signals with specific cell status and morphology for 5 days (Figure 35b). The allplanar OECT configuration, obviating the use of external (sometimes toxic in the case of $\mathrm{Ag} / \mathrm{AgCl}$ ) electrodes, facilitates continuous in situ monitoring of cell cultures in aseptic conditions, minimizing (i) risks for contamination and (ii) cell stress (for example, by moving in and out of the incubator), hence favoring long-term measurements. In addition, this experimental setup facilitated acquisition of electrical signals even before cells are confluent, revealing new information about cell growth and behavior by demonstrating that in MDCK-I tight junctions are formed before the layers reach confluency (Figure 35c)..$^{541}$ In follow-up work, the group further exemplified the benefits of using planar OECTs as cell biosensors by optimizing the device features (e.g., geometry, gate type) for enhanced electrical and optical recordings of barrier tissues, ${ }^{285}$ but also expanded the application to nonbarrier-forming cell layers and showed the principle of use for detecting biological signals from tissues, challenging to pick up by conventional methods including the Transwell OECTs. ${ }^{350}$ Four epithelial cell types, with well-characterized morphology and barrier function (i.e., tight, intermediate, no barrier) were seeded on devices, and the evolution of each culture was monitored for about 10 days.

Figure 36 illustrates images of the devices used in this work, the compatibility for simultaneous optical monitoring in the fluorescent mode, as well as a schematic representation of ion flow pathways and the biological parameters that can be measured in both non-barrier-forming and barrier-forming cell cultures. To correlate the electrical readouts with the relevant biological parameters, the authors used the device's cut-off frequency as a figure of merit, extracted from the bandwidth measurements over a broad range of frequencies. ${ }^{60}$ As seen in Figure 36d,e, electrical monitoring confirmed what is known from literature for the trend that each cell line used follows: the tighter the barrier is, the higher the resistance and hence the lower the cut-off frequency. In fact, the OECTs used were designed to be sensitive enough (fast enough) to detect activity of leaky cell layers (i.e., non-barrier-forming).

An example showcasing this potential of bioelectronic tools is the engineering of an OECT array to model cancer metastasis. ${ }^{537}$ In this study, the researchers took advantage of the multichannel area of the PEDOT:PSS-based OECT array to monitor the barrier integrity of epithelial cells invaded by nasopharyngeal carcinoma. First, they demonstrated the ability of the device to distinguish between different types of cells (i.e., Caco-2 and MDCK epithelial cells vs NP460 and NPC43 cancer cells), as reflected in the distinct frequency-dependent transconductance response and cross-validated with imaging assays (Figure 37a,b). To model invasion of cancer cells in normal epithelial barriers, a coculture of both cell types was seeded on the channel area formed by a $4 \times 4$ matrix of OECTs. By tagging the carcinoma cells with a fluorescent dye to optically follow the cell distribution on the device and by using the frequencydependent transconductance as the electrical signature of each cell type, a heat map was formed to spatially map cancer- 

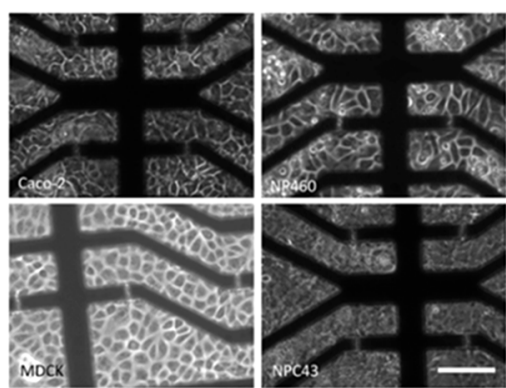

b

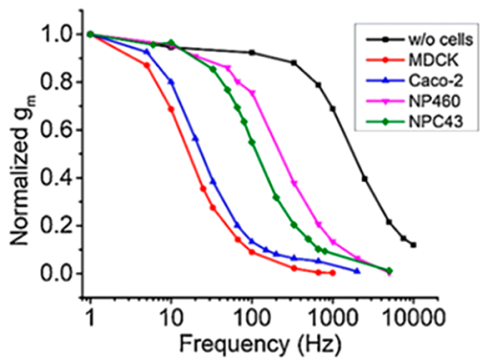

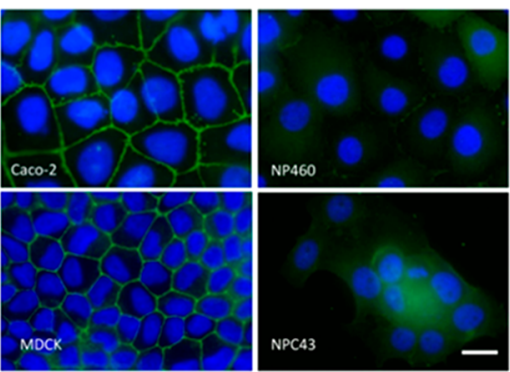

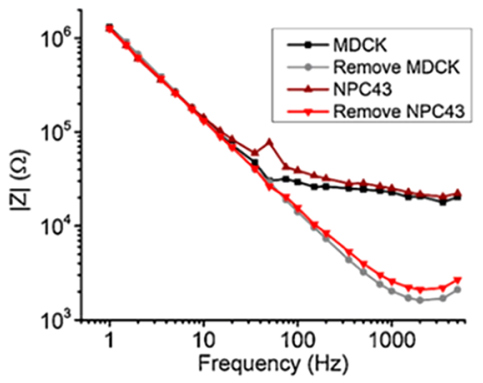

C

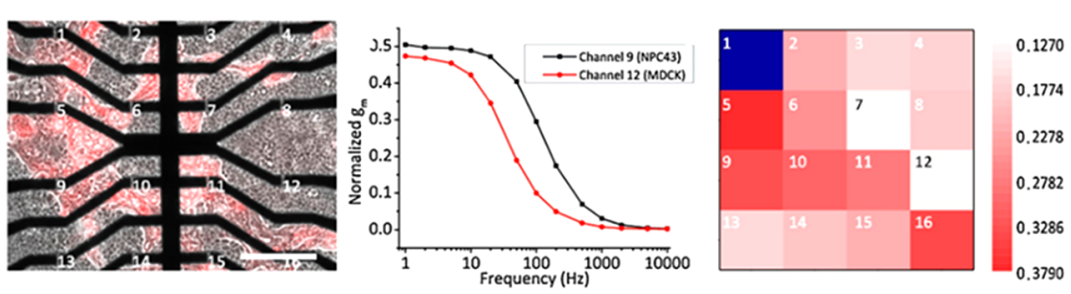

Figure 37. (a) Phase contrast and immunofluorescence images of Caco-2, MDCK, NP460, and NPC43 cells cultured on OECTs (green, ZO-1 tight junctions; blue, cell nuclei; scale bars 100 and $10 \mu \mathrm{m}$, respectively). (b) Plots of normalized transconductance cultured with different cell types and impedance spectra of OECTs in the presence and absence of either MDCK or NPC43 cells. (c) Microscopy image of MDCK and mCherry-tagged NPC43 cell coculture on a 16-OECT array. Normalized transconductance curves of 2 representative channels covered by either MDCK or mCherrytagged NPC43 cells and heat map of the cell distribution on the multichannel area, based on the transconductance value of each channel at $100 \mathrm{~Hz}$. As the intensity of the color increases, the chance that the channel is covered by carcinoma cells is increasing, as reflected by the increase in the corresponding transconductance value at $100 \mathrm{~Hz}$, which is indicative of the presence of a leaky cell. The blue box illustrates a damaged OECT. Reproduced with permission from ref 537. Copyright 2019 Elsevier.

epithelial cell patterns (Figure 37c), offering a new insight into carcinoma invasion dynamics. ${ }^{537}$

A different approach in combining optical with electrochemical sensing was proposed by Löfflera and RichterDahlfors, ${ }^{250}$ who suggested that phase angle spectroscopy (PAS) can offer in-depth analysis of the mechanisms of epithelial cell formation and disruption, highlighting phase angle $\varphi$ as an important parameter of the EIS spectrum for characterizing biological systems. PEDOT:PSS-functionalized ITO electrodes were used to study the formation and disruption of a kidney epithelial barrier via real-time electrochemical sensing combined with optical monitoring of cells. A consistent and characteristic peak in the $\varphi$ amplitude in the $10^{3}-10^{5} \mathrm{~Hz}$ (high) frequency range (Figure 38a) was revealed, attributed to the cell layer capacitive coating of the electrode, as cell density and polarization increases during the formation of the barrier. This was cross-validated by confocal microscopy, which also revealed that the monolayer adopted a polarized phenotype (Figure 38b). Further, this method was able to capture the disruptive effects of the membrane-permeable ionophore ionomycin on the epithelial barrier integrity (Figure 38c). This approach provides a complementary tool for more in-depth in vitro analysis of cellular communication and tissue formation and disruption, among other applications. ${ }^{250}$
Besides the operation mode of the device, the geometry of the device can also be tuned to fit the requirements of specific applications, as it has been well established that the geometrical features of certain bioelectronic devices significantly affect their performance and thus electrical characteristics (e.g., impedance, transconductance; see section 2.4). ${ }^{109,264,542}$ One of the most recent studies that employed this approach was by Hsing and collaborators, ${ }^{283}$ who postulated that variations in the OECT channel dimensions would significantly affect the performance of the devices as cell sensors. To test this hypothesis, the researchers compared the response (i.e., $g_{\mathrm{m}}, \tau$ and $|Z|$ ) of OECTs with different dimensions upon culture with either barrier-forming (Caco-2 human epithelial colorectal adenocarcinoma cells) or non-barrier-forming (NPC43 nasopharyngeal carcinoma cells) cells (Figure 39). Their findings suggest that there is an optimum bandwidth of operation for specific-sized OECTs: large-channel OECTs exhibit higher volumetric capacitance and thus are more sensitive to low frequency currents, such as the ones produced from the paracellular ion flux in barrier tissues; small-channel OECTs are better suited for monitoring activity of leaky adherent cells, where a faster response is required for accurately capturing signals (highfrequency domain). ${ }^{283}$

The potential of bioelectronic devices as tools for in vitro diagnostics and, in particular for toxicology and drug develop- 
a

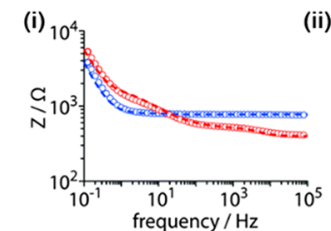

(ii)

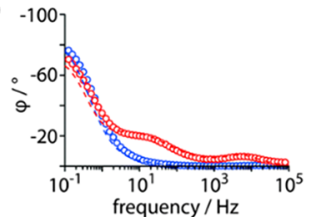

b

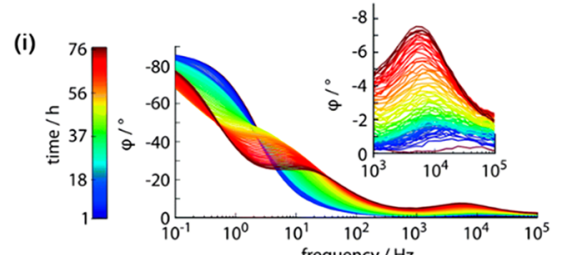

(ii)

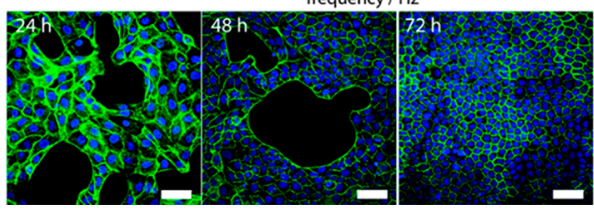

c (i)

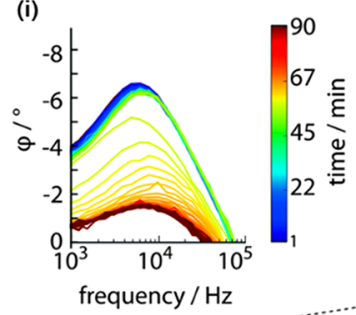

(ii)

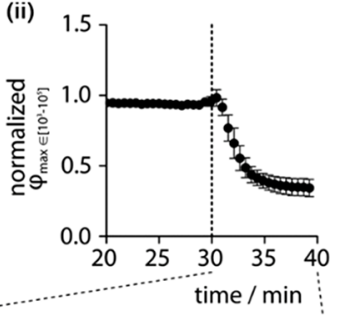

(iii)

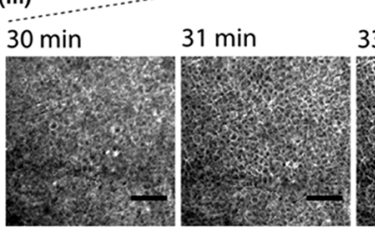

$33 \min \quad 40 \mathrm{~min}$

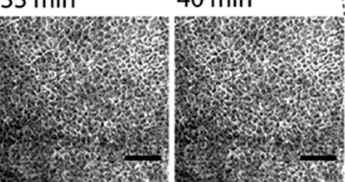

Figure 38. (a) Bode plots of the (i) impedance (Z) and (ii) phase angle $(\varphi)$ of electrodeposited PEDOT:PSS in the absence (blue) and presence (red) of a confluent layer of MDCK cells. (b) (i) Bode plot of the complete PAS spectra of cells growing on the device, color-coded for time (h). Inset illustrates a close-up of phase angle $\varphi$ values in the $10^{3}-10^{5} \mathrm{~Hz}$ frequency range. (ii) Confocal microscopy images of cells growing on the PAS sensor at given time points (green, actin filaments; blue, cell nuclei; scale bar $50 \mu \mathrm{m}$ ). (c) Combined optical and PAS sensing of the disruption of the epithelial barrier by ionomycin. Bode plot of selective, partial spectra of PAS upon exposure of cells to ionomycin after the baseline recording ( 30 min), color coded for time. (ii) Normalized $\varphi$ peak values over time, illustrating the kinetics of epithelial barrier disruption, and (iii) phase contrast microscopy images illustrating the morphology of the barrier at given time points (scale bar $=200 \mu \mathrm{m}$ ). Reproduced with permission from ref 250 . Copyright 2015 Royal Society of Chemistry under Creative Commons Attribution-NonCommercial 3.0 Unported (CC BY-NC 3.0), https://creativecommons.org/ licenses/by-nc/3.0/.

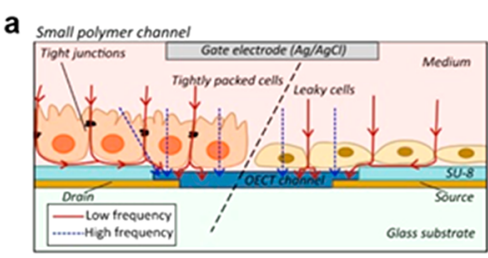

(i)
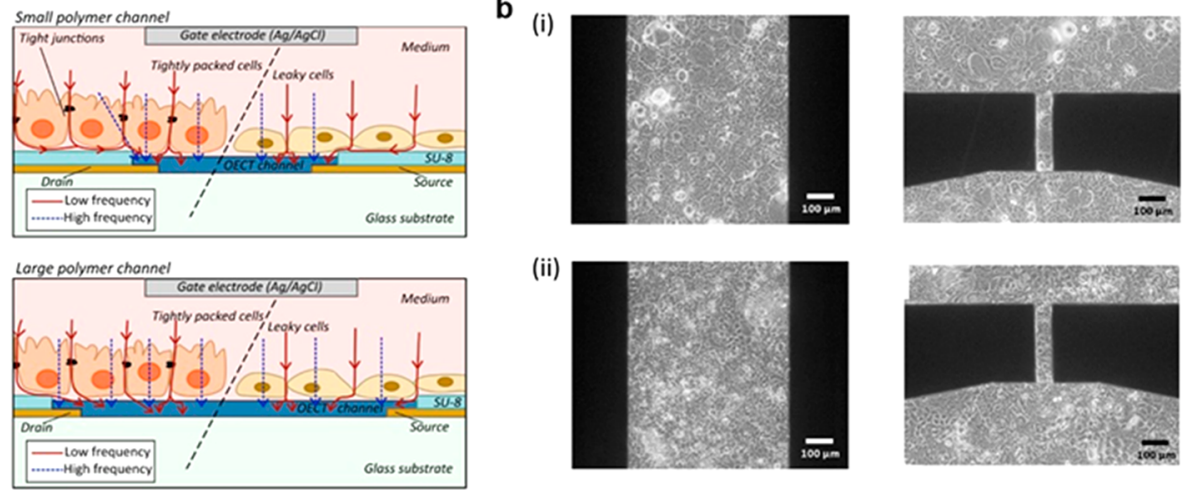

(ii)
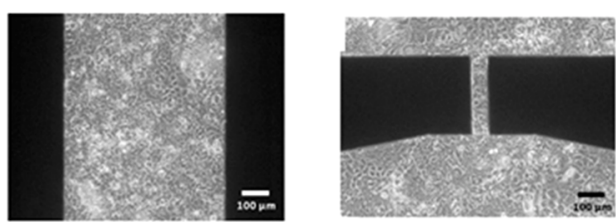

C

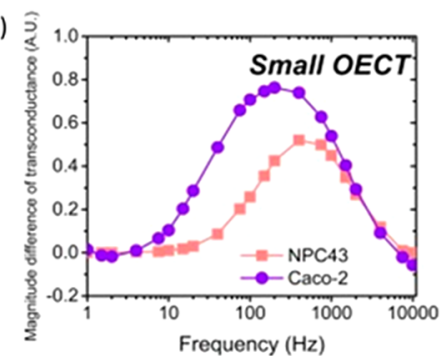

(ii) $\widehat{3}$

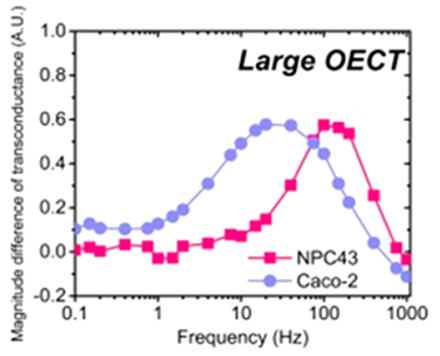

Figure 39. (a) Schematic illustration of OECT devices with different channel areas as sensors of epithelial cell layer integrity, grown on the OECT channel (Caco-2 cells, illustrated as tightly packed cells, or NPC43 cells, illustrated as leaky cells) (top left) were deposited on OECTs in different channel areas. In both cases, arrows indicate the flow of high- and low-frequency currents. (b) Microscope images of (i) Caco-2 and (ii) NPC43 cells growing on large and small channel OECTs. (c) Graphs showing the magnitude difference of transconductance over frequency for small OECT and large OECT, when cultured with Caco-2 or NPC43 cells. Reproduced with permission from ref 283. Copyright 2018 Elsevier.

ment, is without a doubt one of the main reasons this technology has experienced such tremendous growth. This is clearly seen in the applications described in this section, which showcase how bioelectronic tools can be used to investigate the toxicity of certain compounds. ${ }^{275,533}$ However, most studies are limited in the sense that they employ commonly used compounds (e.g., EGTA, $\mathrm{H}_{2} \mathrm{O}_{2}$ ) to mimic phenomena such as inflammation or disruption of barrier tissue to show proof-of-concept. Recent studies though have been focused on taking this a step further. For example, Tria et al. ${ }^{543}$ utilized OECTs coupled with 
(a)

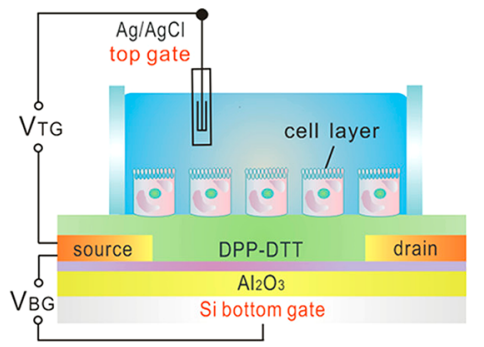

(b)

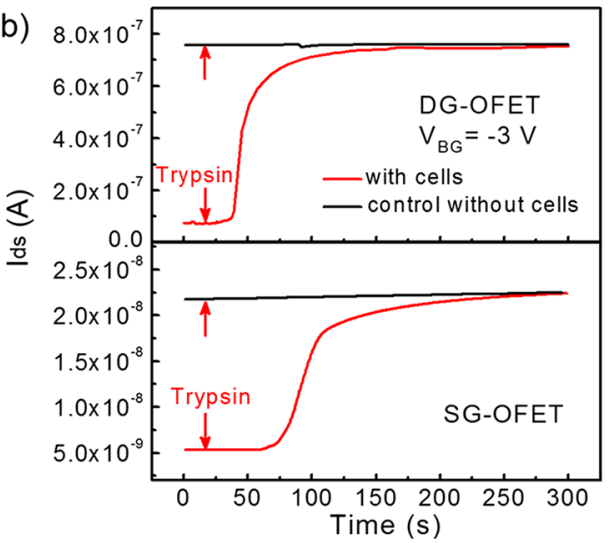

(c)

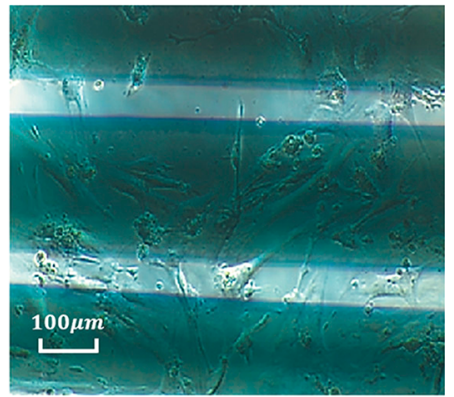

(d)

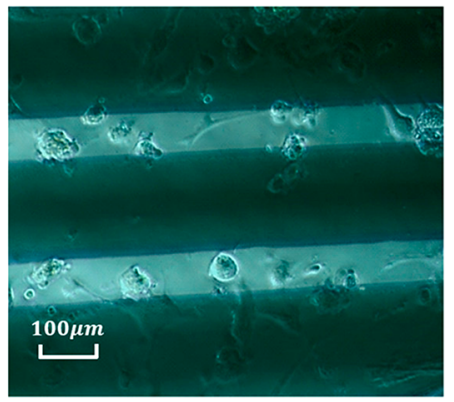

Figure 40. (a) Schematic of the dual gate cell-sensing platform. (b) Current responses over time of the DG-OFET (top) and SG-OFET (bottom) with and without hMSCs upon trypsin treatment. (c, d) Optical images showing hMSCs cultured on the OFETs before and after trypsin treatment, respectively. Adapted with permission from ref 302. Copyright 2017 American Chemical Society.

Transwell filter membranes to dynamically monitor the barrier integrity and to detect infection of polarized intestinal layers by enteric pathogens (i.e., Salmonella typhimurium) under physiological conditions. In addition, the authors demonstrate stable operation of the devices and their ability to detect early and subtle changes in the epithelial barrier resistance with high sensitivity in complex substrates, such as full-fat milk, giving prominence to the powerful potential of OECTs as highthroughput and reliable tools for diagnostics and food and safety applications. ${ }^{543}$ Taking this one step further, Huerta et al. implemented an all planar OECT configuration cultured with the low transepithial resistance (TER) human kidney proximal tubule model, for bandwidth measurements of the barrier tissue integrity in response to a variety of nephrotoxic agents. The cell damage induced by cisplatin and other toxic compounds tested in cell cultures was successfully detected with the OECTs and corroborated via immunofluorescence assays, while not detected with the commercially available system CellZscope. This study paves the way for future integration into microfabricated lab-onchip platforms. In the follow up study, kidney cells (MDCK-II) were cultured in a microfluidic integrated OECT array toward on-chip monitoring of biological activity under physiologically relevant conditions, along with the efficient integration of multiple assays, including metabolite sensing and a woundhealing assay. ${ }^{281}$

More recently, Ferro et al. ${ }^{54}$ developed an OECT that makes use of a conformable gate electrode that can operate in airliquid (ALI) culture environments, simply by placing the electrode on top of the apical cell border without the need for electrolyte-media addition. This configuration allows certain cell types (e.g., lung, skin) to grow and function in a more physiologically relevant environment, while their status and properties can be continuously monitored. The dose-dependent toxicity of e-cigarette aerosols was tested on the integrity of the tracheal epithelial barrier in terms of barrier resistance, offering new insight into the mechanism of bronchial epithelial barrier disruption by inhaled smoke over time and a powerful tool for further toxicology studies. ${ }^{544}$ In another study, OECTs were used to investigate the toxicity of silver nanoparticles (i.e., toxic citrate-coated AgNPs or nontoxic $\mathrm{EG}_{6} \mathrm{OH}$-coated AgNPs) on mouse embryonic fibroblasts (i.e., NIH-3T3) and human colorectal adenocarcinoma cells (i.e., Caco-2). Real-time monitoring of the variation in response time of the current modulation upon application of a square voltage pulse at the gate, cross-validated with immunofluorescence microscopy, showed that OECTs can efficiently and accurately monitor the health status of cells upon such treatments to distinguish toxic from nontoxic nanoparticle effects and to provide detailed information on the kinetics of toxic compounds. ${ }^{545}$

Finally, it is worth noting the efforts to broaden the material assortment and to use more types of devices for in vitro biomedical studies. An interesting example is the case of OECTs based on polydiketopyrrolopyrroles (PDPPs), published earlier this year. The materials exhibited excellent performance and stability in common cell culture media, and preliminary findings suggest that this class of materials is nontoxic and suitable for interfacing with cell cultures. ${ }^{546}$ Attempting to improve the performance of transistors as cell sensors, Zhang et al., ${ }^{302}$ developed a poly $(N$-alkyl diketopyrrolopyrrole-dithienylthieno$[3,2-b]$ thiophene) (DPP-DTT)-based solid-liquid dual-gate organic transistor (Figure 40a). By exploiting the solid-liquid dual-gating, the authors demonstrate that the threshold voltage of the transistor channel can be tuned to exceed the voltage window of state-of-the-art devices to values higher than $0.4 \mathrm{~V}$, thanks to the additional gate that controls the liquid-gated conduction. In fact, these devices outperformed single-gated devices as sensors for the detachment of human mesenchymal stem cells (hMSCs) upon trypsinization.

3.2.3. Nonmammalian Cells. The application of organic electronic devices in vitro also extends beyond mammalian 
systems, although the latter remain the main focus of this review. It is worth noting that applications of OECTs have now expanded to such diverse organisms as plants, fungi, and even diatoms. ${ }^{547-549}$ Bacteria have also been extensively interfaced with organic electronic devices and materials as detailed below. The molecular mechanisms underpinning bacterial infection have been the subject of study over the past two decades. Understanding the full complexity of bacterial infections and subsequently developing robust models that can serve as a tool to test antimicrobials remains challenging. The exploration of the antimicrobial properties of materials and surfaces is also of great importance for biomedical, food and textile industry applications. A recent study highlights the potential of $\mathrm{CP}$ coatings for personal protective equipment (PPE) and medical equipment to ward off secondary bacterial infections in hospital settings, given their current prevalence among COVID-19 patients. ${ }^{550} \mathrm{CPs}$ have been increasingly adopted for in vitro applications with bacterial cells given their innate or electrochemically induced antimicrobial properties as well as their ability to act as electron relays for bacterial extracellular electron transfer. The latter is of great importance not only for understanding and studying bacterial systems but also for developing energy generation/storage devices known as microbial fuel cells. As such, CPs have found widespread applications in antimicrobial surfaces, ${ }^{551}$ antibiotic research, ${ }^{552,553}$ biosensing, ${ }^{554,555}$ and biofuel cells. ${ }^{550}$

3.2.3.1. How Bacterial Cells Are Interfaced with CPs. Bacterial cells have been coupled with CP films using different methods including direct absorption on the CP surface, ${ }^{556}$ entrapment of bacteria in the $\mathrm{CP}$ matrix during polymerization, ${ }^{557}$ realization of bacterial-imprinted CPs, ${ }^{558}$ and finally entrapment of bacterial cells as anionic dopants due to the negative charge of bacteria cell walls. ${ }^{559,560}$ The method used depends on the intended application. For example for the development of robust and long-lasting biosensors, the entrapment and doping approaches provide a more stable alternative. ${ }^{559,560}$ In a broad study, when compared to other materials (Au, ITO, glassy carbon), CPs were found to be more suitable matrices for entrapping bacteria in terms of their density, viability, and distribution. ${ }^{559}$ For fundamental studies, however, for example, applications including biofilm formation studies or testing of antibacterial properties of a given surface, bacterial adsorption has been more widely used. ${ }^{555,561,562}$ Further, the redox active properties of CPs render them particularly suitable for electrical "communication" with bacterial films to modulate or sense bacterial metabolism. ${ }^{561}$

3.2.3.2. Antimicrobial Properties of CPs. Early studies by Seshadri et al. revealed the excellent antimicrobial activity of PANI-based CPs toward both Gram-negative and Grampositive bacteria. ${ }^{563}$ Later Shi et al. showed that PANI blended with PVA restricted the growth of Escherichia coli and Staphylococcus aureus in both dark and light conditions, highlighting its potential to be used as a self-cleaning surface for dark environments where photocatalytic antibacterial agents are ineffective. ${ }^{564}$ Another study with functionalized PANI films extended the antimicrobial spectrum of PANI, exhibiting efficient microbial resistance for wild-type E. coli, Pseudomonas aeruginosa, and $S$. aureus, as well as several antibiotic resistant pathogens. ${ }^{565}$ Since then, various PANI blends or composites including ones with chitosan, zirconium (IV) sulfosalicylate, Ag, and $\mathrm{Au}$ have been explored. PPy has been also found to exhibit intrinsic antimicrobial activity, which was attributed to the presence of positive charges along the backbone chain during its synthesis via oxidative polymerization. ${ }^{566}$ A common strategy to further enhance the antimicrobial activity of PPy (and other polymers) involves the incorporation of antimicrobial agents in the form of metal nanoparticles (i.e., $\mathrm{Ag}^{567,568}$ and $\mathrm{Pd}^{569}$ ), polymers (i.e., chitosan ${ }^{570}$ ), or nanostructures (i.e., CNTs). ${ }^{571}$ The incorporation of nanoparticles increases the number of active sites in the polymer matrix, which in turn enforces the electrostatic interactions with the bacteria cell wall. ${ }^{572}$ In the case of CNTs, the underlying antimicrobial function arises from the oxidative damage to the lipid membrane, though the effect depends on the type of CNTs or their functionalization type. ${ }^{573}$ The influence of morphology and additives on the antibacterial behavior of PPy was demonstrated in a systematic study by $\mathrm{Da}$ Silva et al. ${ }^{556}$ Specifically, the authors tested various PPy-based systems: conventional PPy, branched PPy, branched PPy/Ag nanocomposite, highly soluble PPy, and PPy/Ag colloid. The highly soluble PPy exhibited the best bactericidal efficiency against E. coli, Klebsiella pneumoniae, and S. aureus. While the rest of the systems showed a bacteria-dependent effect, the PPy/Ag colloid and the branched PPy/Ag were found to encounter effectively the bacteria with acceptable characteristic-time kill. ${ }^{556}$ The antimicrobial efficacy of PEDOT based systems has been also explored. ${ }^{562,574,575}$ In a recent study, Alemán's team investigated the response of $\mathrm{n}$-doped PEDOT films against E. coli and $S$. aureus bacteria. ${ }^{562}$ The incorporation of an ndopant (pyridinium-based polyelectrolyte) was found to promote the bacteriostatic behavior of PEDOT without inducing toxicity to eukaryotic cells (NRK and Vero cells). A recent work demonstrated the use of nanocomposites based on functionalized PEDOT and AgNPs as a coating for stainless steel surfaces. ${ }^{576}$ Specifically, PEDOT functionalized with zwitterionic phosphorylcholine was found to inhibit the bacterial ( $E$. coli and Streptococcus mutans) adhesion, while the presence of AgNPs further prevented their colonization.

3.2.3.3. Controlling Bacterial Growth. The availability of suitable electron acceptors is critical to bacterial growth and, therefore, the establishment of a biofilm. A comprehensive study on the effect of the redox state of CPs on bacterial growth and biofilm formation was recently conducted by Richter-Dahlfors and co-workers. ${ }^{561}$ By electrical oxidation or reduction of a PEDOT composite, the availability of electron acceptors could be controlled providing an electron sink that promotes bacterial metabolism and hence biofilm growth. In contrast, reduced PEDOT was found to exhibit antifouling activity (reducing bacterial growth). Those two opposing mechanisms observed in this study suggest that the electrochemical state of CP surfaces can be fine-tuned to either prevent bacterial growth (i.e., in the healthcare sector, in food processing, in paper manufacturing) or favor the growth of bacteria (i.e., for fundamental studies of bacterial metabolism or the development of antibacterial therapies).

3.2.3.4. Sensing Bacterial Metabolism. CP films (PEDOT and PPy) have been used for fundamental studies on the metabolic activity of bacterial cells, in particular for the electrochemical evaluation of the respiratory activity of anaerobic and aerobic bacterial cells. ${ }^{560}$ Typically, metabolic activity of bacterial cells is assessed through their growth and respiration patterns via cell-counting or staining or gas chromatography all being indirect and time-consuming methods. ${ }^{577,578}$ The ability to easily quantify bacterial metabolism (i.e., with CPs) is thus of importance for a variety of applications including testing of antibiotics, as there is a link 


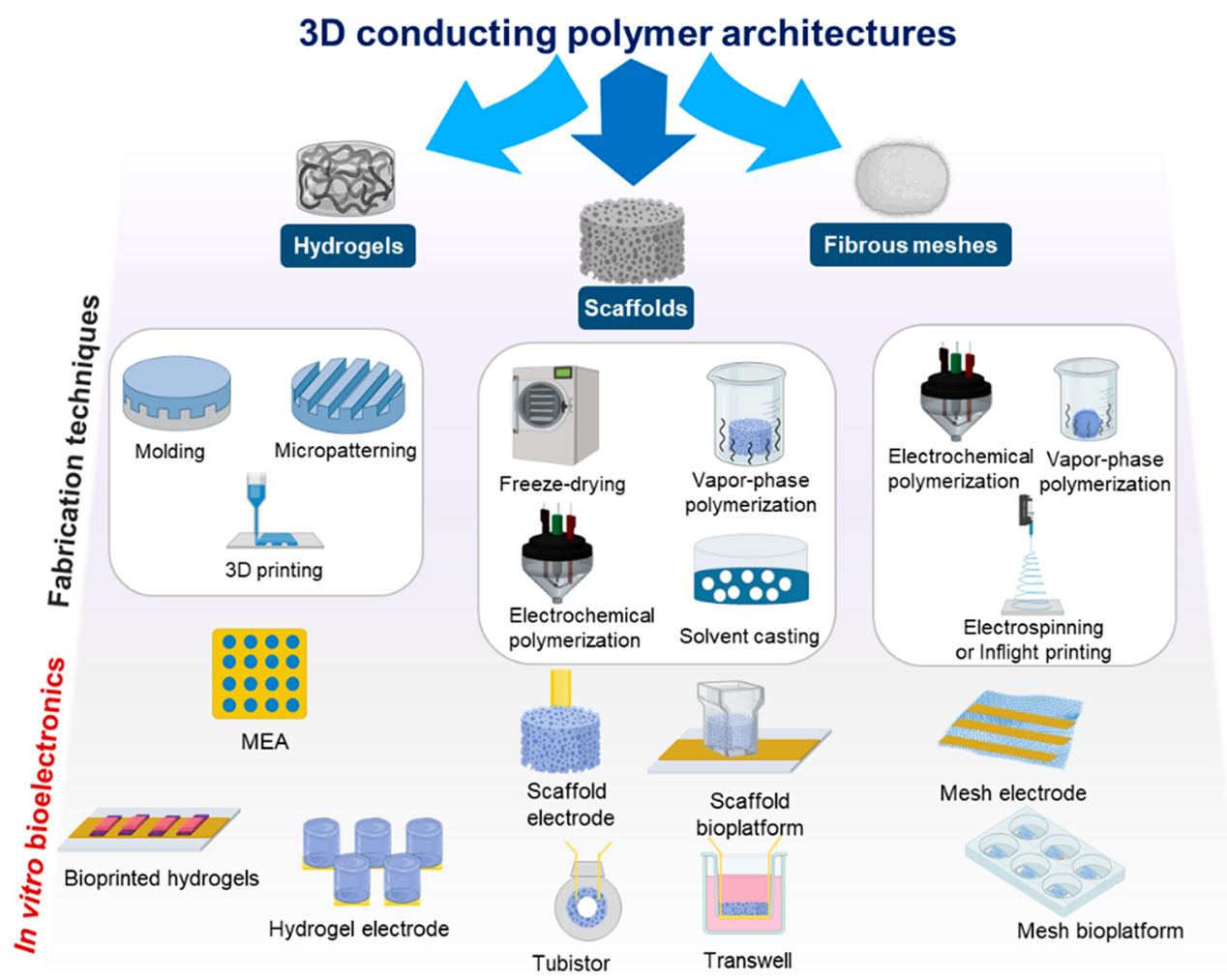

Figure 41. Schematic illustrates the main 3D conducting polymer architectures used in bioelectronic interfaces with 3D cellular systems in vitro. Created with Biorender.

between the metabolic state of bacterial and the antibiotic efficacy. ${ }^{579}$

3.2.3.5. Biofuel Cells. It is well-known that under anaerobic conditions, certain bacteria produce protons and electrons instead of carbon dioxide and water. This attribute of bacterial cells has been heavily exploited for applications in energy production and storage. The first microbial fuel cell was realized in the beginning of the 20th century when scientists connected bacterial cells with electrodes to generate electricity. While the potential of this is high, the main drawback is the low power density as the generated electrodes are not efficiently transmitted to the electrode. Hence, CPs, having the ability to act as electron relays, have shown that they can greatly enhance the efficiency of this process. ${ }^{580} \mathrm{Li}$ et al. investigated four kinds of CPs including PANI and three derivatives as coatings of conventional electrodes, acting as aerobic abiotic cathodes and biocathodes in microbial fuel cells. They found that all four polymers significantly improved the power densities for both abiotic cathodes (increased by 300\%) and biocathodes (increased by $180 \%) .{ }^{581} \mathrm{CPs}$ ' good oxygen catalytic reduction ability and favorable biofilm matrix render them especially promising materials for next generation biofuel cells.

\subsection{D Cell Cultures}

To recapitulate biological systems in vitro, it is important to generate $3 \mathrm{D}$ models that better reflect the in vivo situation. Organs, tissues, and support networks such as the extracellular matrix (ECM) have complex architectures, being naturally organized in $3 \mathrm{D}$ structures rather than in $2 \mathrm{D}$ structures. Although the use of $2 \mathrm{D}$ cell culture systems has facilitated the discovery and progress of various fields in biomedical research, ${ }^{582,583}$ they lack the environmental context and in vivo architecture, and combined with the absence of biophysical cues and of a heterogeneous population of cells, they only represent a simplistic imitation of the in vivo situation. ${ }^{22,582,584}$ As a result, this may lead to significant differences in the response to potential drugs and treatments and to misleading conclusions about the safety and efficacy of such compounds. ${ }^{585,586}$ In this regard, quasi-3D (or 2.5D) models emerged as a transitional stage to model cell biology. Cells in 2.5D models are cultured on top of substrates, in some cases coated with ECM proteins to enhance cell adhesion and differentiation. ${ }^{544}$ Substrates with diverse patterns and topographies can help in representing natural cell organizations to obtain physiologically relevant information about cell behaviors.

Over the last decades, the field is progressively transitioning into truly $3 \mathrm{D}$ models that more accurately mimic the in vivo complexity. ${ }^{583,587-589}$ Benefiting from the synergy of multiple disciplines, including cell biology, materials science and engineering, and the significant advances in tissue engineering, novel 3D cell culture technologies, which can be tailored to meet the requirements of a specific application, have been brought to the fore.

Cell culture models interfacing with bioelectronics include tissue-engineered structures such as hydrogels, porous scaffolds, and fiber meshes, as well as more complex $3 \mathrm{D}$ cell models such as spheroids and organoids. Three-dimensional bioelectronic models, in addition to providing a 3D structure for cell support, are also used to electrically monitor cell growth and tissue formation. However, sample handling and imaging, as well as high-throughput, remain a challenge in $3 \mathrm{D}$ structures, requiring more sophisticated technological tools for tackling the third dimension. Downstream analysis of sample effluents (e.g., metabolomics, genomics) is another important aspect and is often performed with end-point assays requiring labeling or the use of invasive probes. 590 
a

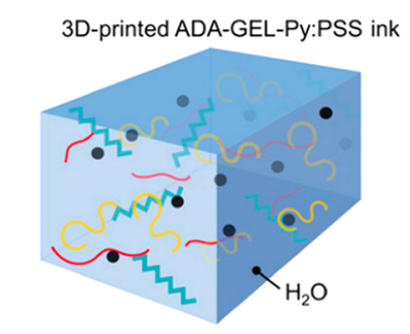

Polypyrrole (PPy) network formation

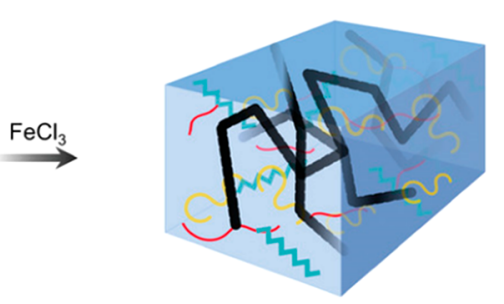

Crosslinked conductive hydrogel
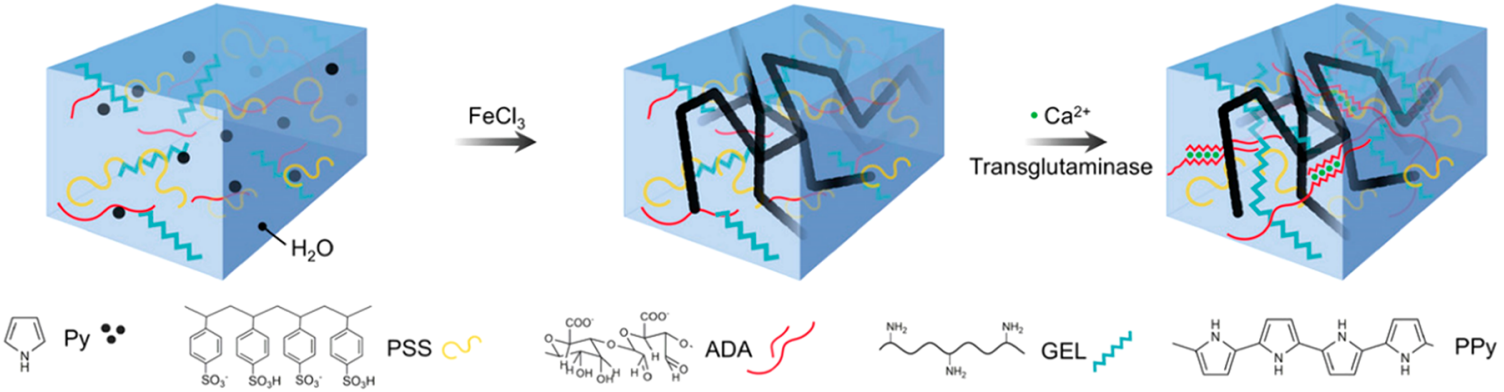

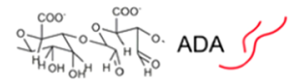

$\overbrace{\mathrm{NH}_{2}}^{\mathrm{NH}_{2}} \mathrm{GEL} \mathrm{N}^{\mathrm{NH}}$

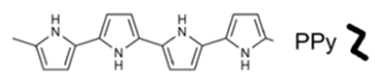

C

b

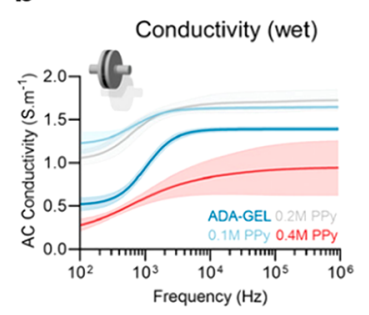

Conductivity (dry)

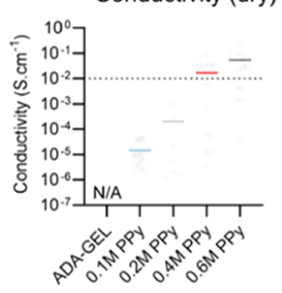

3D AG-PPy:PSS

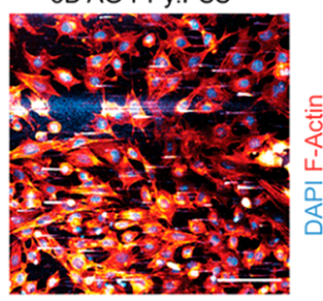

d

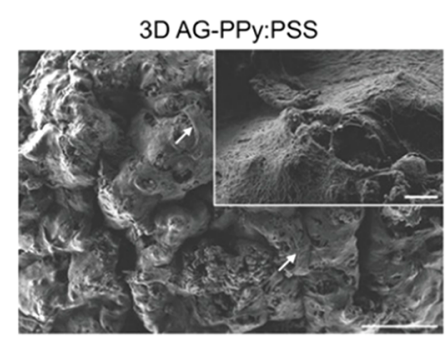

Figure 42. (a) Schematic showing the synthesis route for the development of alginate-gelatin-polypyrrole:PSS (ADA-GEL-PPy:PSS) hydrogels. (b) Conductivity data for wet (AC) and dry ADA-GEL and ADA-GEL:PPy (with various concentrations of PPy, 0.1, 0.2, and 0.4 M) (c) Multiphoton fluorescence microscopy (nuclei (DAPI), blue; F-actin (rhodamine phalloidin), red; scale bar $100 \mu \mathrm{m}$ ) and (d) SEM image of ATDC-5 cells cultured on 3D AG-PPy:PSS hydrogels. Adapted with permission from ref 613. Copyright 2021 Wiley-VCH Verlag GmbH \& Co. KGaA, Weinheim.

3.3.1. Tissue Engineering Approaches. Three-dimensional structures can be used to recreate the same biologypolymer interfaces that were described in sections 3.1 and 3.2 , having excellent electrical and mechanical properties, high porosity and surface area, and biological mimicry. As for their 2D counterparts, they can be adopted to deliver electrical or optoelectronic stimuli to cells or used as bioelectronic interfaces to monitor cellular processes and tissue development in vitro. They also allow the inclusion of physiologically relevant polymers in the form of blends, dopants, and composites to design 3D CP-based structures that feature enhanced biocompatibility. Moreover, the incorporation of growth factors, minerals, and chemical molecules can positively affect cell growth and proliferation.

As defined by Mecerreyes and co-workers, ${ }^{591}$ for CP-based cell models to be considered 3D, they have to comply with three limits of tridimensionality: (a) the length, width, and thickness of the structure have to be higher than the size of the cell under study (micrometer scale), (b) they should have a pore size that allows cell infiltration, homogeneous distribution, and percolation, and (c) they should be conducting throughout their volume. Overall, 3D conducting structures based on their morphological and structural aspects can be categorized as hydrogels, porous scaffolds, and fiber meshes. So far, most of the work in 3D CP-based structures lacks electrical output data from the interface between the CPs and the biological system, resulting in stand-alone passive cell culture systems where cellular events are monitored by biochemical assays and microscopy (e.g., confocal microscopy). An illustrative summary is shown in Figure 41, and a detailed discussion of each hydrogel, porous scaffold, and fiber mesh fabrication process follows, emphasizing in vitro bioelectronic interfacing.

3.3.1.1. Hydrogels. Hydrogels are polymeric networks with high water content and a degree of flexibility that, together with their soft nature, represent ideal scaffolding materials for 3D cell culture applications. Incorporation of CPs into hydrogels interfaced with tissues allows their use as electrodes, for electrical stimulation or monitoring ${ }^{52,593}$ or to achieve controlled release of drugs. ${ }^{594-596}$ CPs can be incorporated as a blend, ${ }^{597}$ cross-linked with the main polymeric backbones, ${ }^{598}$ self-assembled without the addition of cross-linkers, ${ }^{599}$ or the main component of the hydrogel. ${ }^{600}$ The strengths and weaknesses of the generation of conducting hydrogels have been reviewed recently. ${ }^{601}$ Most components used in hydrogels are biopolymers, such as cellulose, ${ }^{602-604}$ gelatin, ${ }^{605}$ chitosan, ${ }^{478,606-608}$ or alginate, ${ }^{248}$ that provide additional biocompatibility and polymers, such as PEG $^{609}$ and acrylates, ${ }^{610}$ that facilitate hydrogel processing. Special attention is paid to the way the use of insulating polymers can affect the conducting properties of the hydrogel. ${ }^{61}$ Moreover, carbon-based materials such as CNTs and multiwalled CNTs (MWCNTs) can be added to enhance the electrical properties and introduce nanotopography in hydrogels. ${ }^{604}$

CP hydrogels can be processed by molding, ${ }^{598}$ micropatterning, ${ }^{609}$ and 3D printing, ${ }^{612,613}$ among other fabrication techniques, in order to trigger specific cell behavior and functionality. Commonly, CP mixtures are dispersed inside cavity-based structures, such as wells or molds, to be polymerized and create molded hydrogel structures. ${ }^{59}$ As an example, molded cellulose hydrogels obtained from pristine tunic sea squirts (marine invertebrate animals) were in situ polymerized to create PPy tunic hydrogels that exhibited suitable conductivity and mechanical properties. Additionally, micropatterned hydrogels composed of a patterned PEGhydrogel polymerized with PEDOT were developed to electrically stimulate the differentiation of myoblast cells. ${ }^{609}$ Myoblasts were seeded onto microgrooved hydrogels showing good cell adhesion and proliferation and elongated morphology in line with hydrogel alignment. Hydrogels based on the combination of oxidized PPy:PSS and an oxidized alginate- 
gelatin (ADA-GEL) hydrogel with a high gelatin content were developed for cartilage regeneration. ${ }^{613}$ The ADA-GEL-Py:PSS hydrogels were $3 \mathrm{D}$ printed and functionalized with $\mathrm{FeCl}_{3}$ to form the conductive PPy:PSS inside the ADA-GEL. (Figure 42a). ADA-GEL and ADA-GEL-PPy:PSS formed with oxidized $0.1,0.2$, and $0.4 \mathrm{M}$ Py were characterized both wet and dry by EIS (Figure 42b). ADA-GEL-PPy:PSS hydrogel cytocompatibility was investigated by seeding mouse teratocarcinoma ATDC-5 cells and evaluated via multiphoton fluorescence microscopy (Figure 42c) and SEM (Figure 42d). Both data sets revealed that cells proliferate in the $z$-direction of the $3 \mathrm{D}$-printed hydrogel due to the strut-by-strut structure of the hydrogel.

Regardless of the advances made in the CP hydrogel field, very few examples of CP hydrogels interfacing with bioelectronic devices for in vitro applications can be found in literature. A strategy to enhance the interface between devices and cells is to create electrodes based on CPs coated with a thick biopolymeric hydrogel to reduce the mechanical mismatch at the electrodetissue interface and improve the electrochemical properties. In this context, Ghezzi and co-workers developed an MEA device based on PEDOT:PSS and coated it with an alginate hydrogel for the electrophysiological recording of neural stem cell differentiation of iPSCs. ${ }^{248}$ The hydrogel was electropolymerized on top of various MEAs (Figure 43a) and then

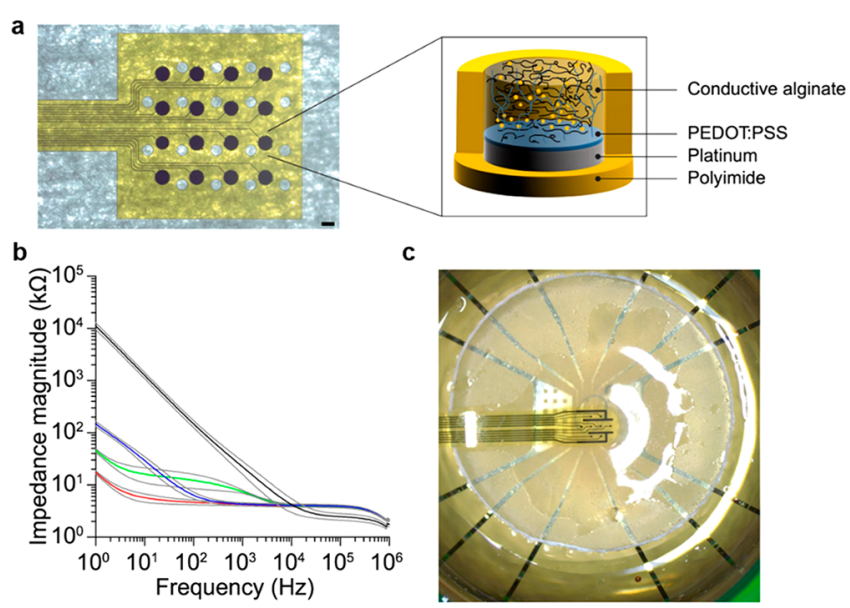

Figure 43. (a) Grid-MEA (g-MEA) fabricated with $400 \mu \mathrm{m}$ diameter electrodes. (b) Bode EIS plots measured at each fabrication step: platinum (black); PEDOT:PSS (blue); alginate (green); conductive alginate (red). Colored lines represent average while gray lines represent SD. (c) Picture of the setup with p-MEA (top device), NS (middle hydrogel), and s-MEA (bottom device). Adapted with permission from ref 248. Copyright 2018 the Authors, licensed under Creative Commons Attribution 4.0 International (CC BY 4.0) License, https://creativecommons.org/licenses/by/4.0/.

characterized by EIS (Figure 43b), showing a decrease of the overall impedance magnitude with the addition of PEDOT:PSS, alginate, and conductive alginate. For the in vitro validation, MEAs were coated with neurospheres made of alginate hydrogels and neural stem cells from iPSCs. (Figure 43c). Electrophysiology recordings showed that the conductive alginate microelectrodes demonstrated lower impedance magnitude, higher charge storage capacity, and a more resistive behavior translating to an improved SNR ratio during neuronal recordings.

3.3.1.2. Porous Scaffolds. Porous scaffolds are 3D polymeric interconnected pore networks that host cells to create $3 \mathrm{D}$ cell cultures. The sufficient porosity and water intake facilitates percolation and infiltration of the cells, rendering such constructs ideal for the spatial 3D organization of cell cultures. Three-dimensional CP porous scaffolds have been mainly used as passive tissue substrates for hosting cell growth and differentiation by biochemical means. ${ }^{52}$ In the last years, their use as active interfaces has enabled electrical monitoring of cell growth and proliferation ${ }^{41}$ and cell differentiation, through electrical stimulation. ${ }^{614}$ Alongside interfacing cardiac and brain tissues, ${ }^{615,616} 3 \mathrm{D} \mathrm{CP}$ porous scaffolds have been used to interface various cell systems derived from bone, ${ }^{51,52}$ kidney, ${ }^{41,53,617}$ brain, ${ }^{618,619}$ and gut. ${ }^{40} \mathrm{CP}$ based porous scaffolds can be fabricated directly via the freeze-drying technique (also known as lyophilization or ice-templating), $39-41,51-53,617,620$ or indirectly by coating polymeric porous templates by vapor phase polymerization (VPP), ${ }^{621}$ solvent casting, ${ }^{618}$ and electrochemical polymerization. ${ }^{591,622,623}$ The most common fabrication method is the direct in situ freeze-drying process, which comprises three steps. First, the desired CP mixture is immersed into dedicated molds, which determine the resulting shape and structure of the scaffold. Second, the mixtures are frozen at a specific cooling rate. Third, the scaffolds are dried at a controlled pressure to sublime the generated water ice crystals and to obtain a dry scaffold. ${ }^{51}$ Scaffold composition, cooling rates, and freezing temperature can influence the porosity and the pore size, as well as the final mechanical properties of the scaffold. Sajesh and co-workers first demonstrated conducting scaffolds alginate-functionalized PPy incorporated into a chitosan matrix. ${ }^{624}$ In this work PPy was obtained by chemical oxidative polymerization of a pyrrole solution containing alginate in the presence of $\mathrm{FeCl}_{3}$. The formed blend was then lyophilized, and the resulting scaffold was cross-linked with glutaraldehyde. Later Wan et al. demonstrated the fabrication of electrically conducting mesoporous PEDOT:PSS scaffolds capable of supporting $3 \mathrm{D}$ cell cultures. ${ }^{50}$ Interestingly, performing electrical stimulation of the scaffolds enabled the adsorption of ECM proteins, which aid the adhesion of pro-angiogenic secretion of 3T3-L1 cells in the scaffolds. Similarly, the Stevens group demonstrated the use of PEDOT:PSS porous scaffolds for the culture of MC3T3-E1 osteogenic precursor cells. ${ }^{51}$ (Figure 44a) The resulting cultures were found to enhance the deposition of mineralized ECM and differentiate into mature osteoblasts, rendering PEDOT:PSS scaffolds promising candidates for bone regeneration applications. It is well-known that mechanical properties and cytocompatibility and are very important for the successful proliferation, growth, and differentiation of cell cultures. Thus, many studies on CP-based scaffolds focused on the incorporation of biopolymers such as collagen, ${ }^{41,52}$ gelatin, ${ }^{625,626}$ alginate, ${ }^{626,627}$ chitosan, $^{628}$ silk fibroin, ${ }^{629}$ and cellular-based gels, such as cardiogel. ${ }^{455}$ Indeed, recent studies by our group demonstrated the use of PEDOT:PSS scaffolds containing collagen for enhancing the mechanical properties and promoting differentiation of neural crest stem (NCS) cells. ${ }^{41,52}$ Specifically, scaffolds containing various concentrations of collagen were characterized by electrochemical and mechanical means, which revealed that an increment in the collagen concentration impacts the electrochemical properties of the scaffold, shifting the overall impedance magnitude to higher values (Figure $44 \mathrm{~b}(\mathrm{i})$ ), whereas the scaffold stiffness decreases (Figure $44 \mathrm{~b}(\mathrm{ii})$ ). Neural crest stem cells were tested for their ability to differentiate into bone cells when colonizing the PEDOT:PSS and collagen scaffolds. Cells were seeded onto the different scaffolds and cultured in 

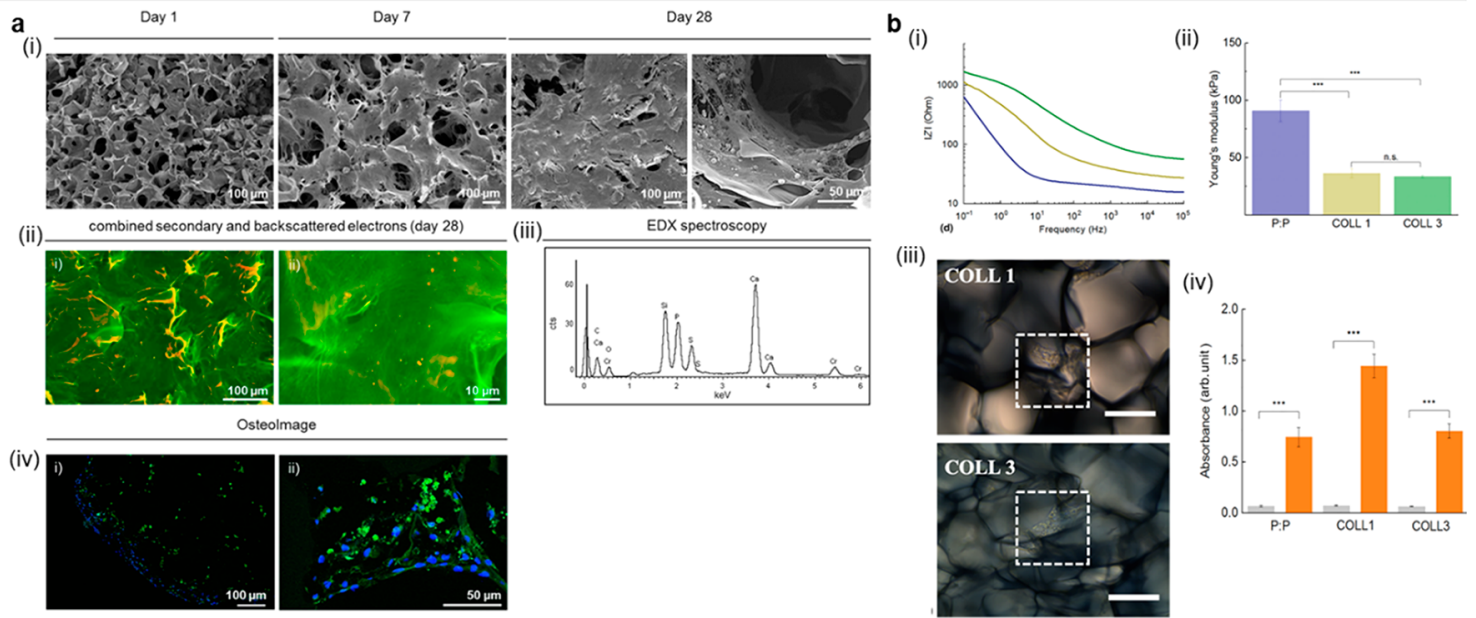

Figure 44. (a) (i) SEM images of PEDOT:PSS scaffolds culture with MC3T3-E1 at different time points (1, 7, and 28 days). (ii) SEM image (falsecolored) of backscattered and secondary electrons showing calcified particles (in red) and organic material (in green) and (iii) EDX spectrum of the MC3T3-E1 cell cultured PEDOT:PSS scaffolds. (iv) Confocal microscopy images showing the cultured scaffold with mineralized bone nodules (in green) and nuclei (in blue-DAPI). Adapted with permission from ref 51. Copyright 2017 Elsevier. Licensed under Creative Commons Attribution 4.0 International (CC BY 4.0) License, https://creativecommons.org/licenses/by/4.0/. (b) (i) EIS Bode plot measured for PEDOT:PSS (P:P, blue line), PEDOT:PSS/collagen-0.3\% (COLL1, yellow line), and PEDOT:PSS/collagen-1.1\% (COLL3, green line). (ii) Young modulus measurements for the various scaffolds. (iii) Brightfield images of the NCSC cultured scaffolds for the different collagen concentrations and (iv) quantitative evaluation of alizarin red dye recovered from the cultured scaffolds after 21 days showing the osteogenic differentiation of NCSCs. Adapted with permission from ref 52. Copyright 2020 Materials Research Society. Licensed under Creative Commons Attribution 4.0 International (CC BY 4.0) License, https:// creativecommons.org/licenses/by/4.0/.

osteoinductive media (Figure 44b(iii)), with scaffolds with less collagen concentration displaying higher levels of alizarin red staining, an indicator of deposited mineralized matrix (Figure $44 \mathrm{~b}(\mathrm{iv}))^{52}$

Biocompatibility in 3D CP-based scaffolds can be also achieved by synthesizing dispersions made of a conducting monomer and a polyelectrolyte by chemical oxidative polymerization. In this regard, different dispersions based on EDOT and various biopolymers such as hyaluronic acid, chitosan, and xantham gum have been developed. Specifically, PEDOT:xanthan gum dispersions were synthesized resulting in scaffolds that were used for hosting MDCK II cells and for monitoring electrically their growth. ${ }^{617}$ (Figure 45a). SEM imaging of the $\mathrm{PEDOT} /$ xanthan gum scaffold shows the high interconnectivity of the porous network (Figure 45b). In terms of electrical monitoring, in the presence of MDCK-II cells after 7 days of culture (Figure 45c) an increase in the impedance magnitude is observed. Confocal imaging (Figure 45d) on MDCK-II eGFP revealed good cell attachment and proliferation within the porous structure.

The addition of biopolymers to CP scaffolds could result in compromised electrical properties compared to the pristine $\mathrm{CP}$ scaffold. Along these lines, incorporation of additives that enhance conductivity, such as CNTs, ${ }^{39,400,618}$ has proven a good strategy to boost the electrochemical properties of the scaffold. In a recent study, different ratios of PEDOT:PSS/MWCNT scaffolds were prepared by the freeze-drying technique and characterized by EIS. The presence of the MWCNTs at 2:3 ratio exhibited the most notable improvement in the conductivity of the scaffolds (Figure 46a(i)). Poly(L-lysine) (PLL)-fluorescein isothiocyanate (FITC) was used to functionalize the scaffolds by means of electrostatic interactions between the positively charged PLL and the negatively charged oxidized MWCNTs. The green staining in the confocal image (Figure 46a(ii)) indicates the presence of MWCNT hot spots as confirmed by the SEM image (Figure 46a(iii)). ${ }^{39}$
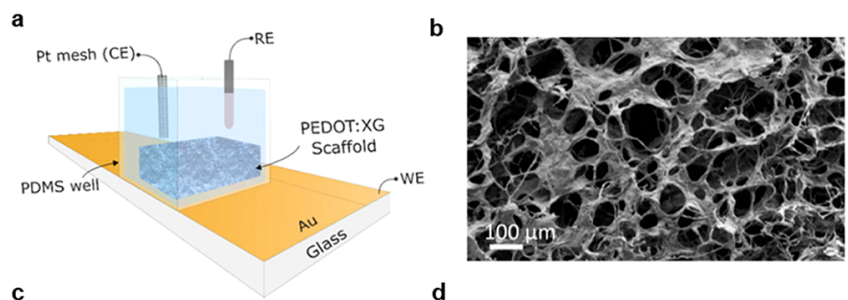

d
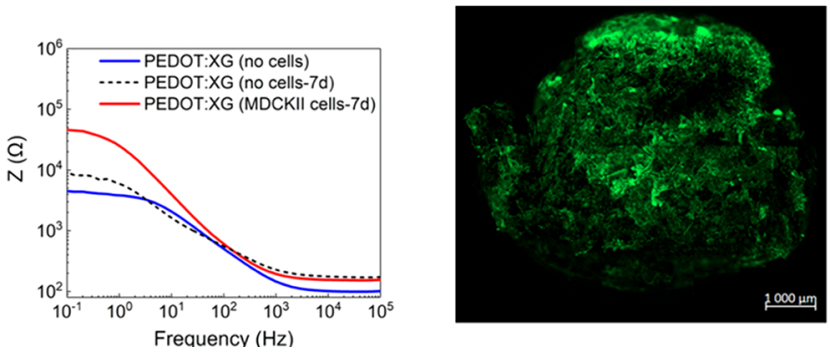

Figure 45. (a) Scaffold-based electrode configuration. (b) SEM image of PEDOT/xanthan gum scaffold (0.5:0.5). (c) Complex impedance data of uncultured and cultured scaffold after 7 days. (d) Confocal microscopy image of the scaffold 14 days after cell seeding. MDCK II eGFP cell growth on PEDOT/xanthan gum (0.5:0.5) after 14 days culture. Adapted with permission from ref 617. Copyright 2018 American Chemical Society. Licensed under Creative Commons Attribution 4.0 International (CC BY 4.0) License, https:// creativecommons.org/licenses/by/4.0/.

Prato and co-workers followed a different strategy to enhance the electrical properties of the scaffolds. In their case, sucrose and CNT templates were electrochemically polymerized using EDOT monomer. Sucrose was removed by submerging the scaffold in water overnight (Figure 46a(i)). The PEDOT/CNT scaffolds were used as templates for differentiation of SH-SY5Y cells (neuroblastoma cells) to a more neuron-like phenotype characterized by neuronal biomarkers. Specifically, $\beta$-tubulin class III and MAP II are neuronal biomarkers that are expressed 
a (i)

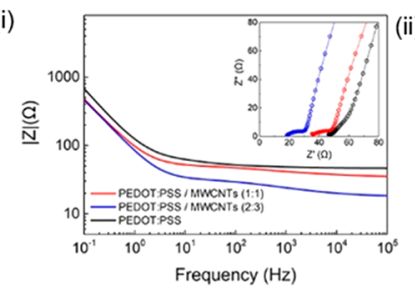

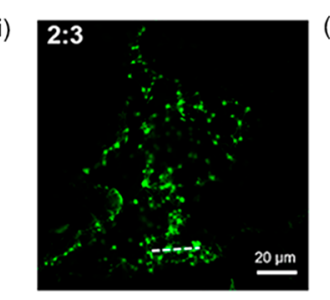

b

(i)

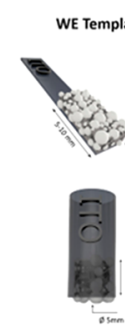

(iii)

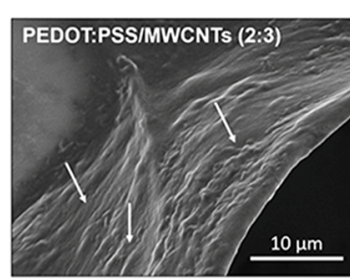

(iv)

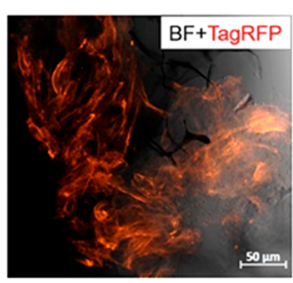

(ii)

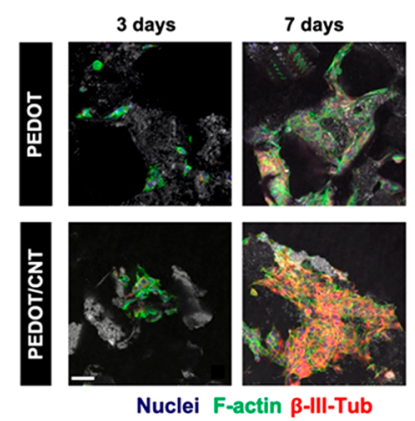

(iii)

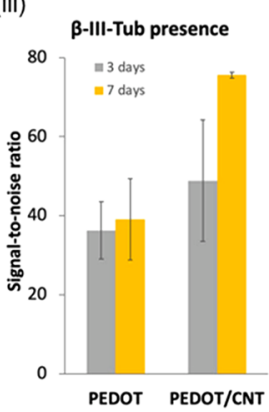

Figure 46. (a) (i) EIS measurements of the PEDOT:PSS and PEDOT:PSS/MWCNT scaffolds (inset shows the Nyquist plots). (ii) Confocal microscopy images of the PLL-FITC functionalized PEDOT:PSS/MWCNT (2:3) scaffolds. (iii) SEM image of PEDOT:PSS/MWCNT (2:3). CNT domains are indicated with white arrows. (iv) Bright-field and far-red merged highlighting the F-actin filaments of TIF cytoskeleton (orange). Adapted with permission from ref 39. Copyright 2019 the Authors. Licensed under Creative Commons Attribution 4.0 International (CC BY 4.0) License, https://creativecommons.org/licenses/by/4.0/. (b) (i) Schematic illustration showing the fabrication technique for the PEDOT/CNT scaffolds. (ii) $\beta$-Tubulin class III and F-actin staining of the SH-SY5Y cells cultured on the PEDOT and PEDOT/CNT scaffolds for 3 and 7 days. (iii) Graph showing the amount of $\beta$-III-Tub measured from the signal-to-noise ratio of immunofluorescence images of the cultured cells. Adapted with permission from ref 400. Copyright 2020 American Chemical Society.

after differentiation of SH-SY5Y cells. Immunofluorescence staining confirmed the hypothesis that cells seeded onto PEDOT/CNT expressed more $\beta$-tubulin class III (Figure $46 \mathrm{~b}($ ii) $)$ and MAP II compared to the PEDOT scaffolds after 7 days. $^{400}$

Electroactive scaffolds can be also fabricated by in situ polymerization of an already prepared scaffold by VPP. This technique allows the coating of the scaffolds in a controllable manner by vaporization of a conducting monomer. The scaffolds are previously soaked into an oxidant solution, placed inside a closed chamber or contained with the monomer solution, and finally, the polymerized scaffold is rinsed with a friendly solvent to remove any unreacted oxidant and monomer. ${ }^{630}$ Simons and co-workers coated polycaprolactone (PCL) scaffolds with PEDOT:Tos using VPP. The scaffolds were seeded with fetal mesenchymal stem cells, and after 14 days no differences between the coated and uncoated scaffolds were observed, suggesting that the coating did not affect cytocompatibility. ${ }^{621}$ VPP has also been used to coat thermoplastic polyurethane scaffolds with PEDOT. The resulting scaffolds exhibited improved mechanical properties compared to their pristine version and good electrical properties. MC3T3-E1 cells were cultured inside the scaffold showing their suitability for muscle and nerve electrical stimulation. ${ }^{631}$ Other approaches include the use of $3 \mathrm{D}$ substrates made of nonconducting polymers as sacrificial templates for the CP coating. ${ }^{618,632-634}$

Although some of the aforementioned in vitro bioelectronic models offer in-line electrical monitoring, they do not accurately replicate the in vivo situation where organs are perfused. Threedimensional CP cell culture platforms can be modeled to accurately represent the perfusion of nutrients of specific organs and tissues. In this context, our group has developed a transistorin-a-tube ( $a k a$ tubistor), a platform that adopts a tubular shape and supports cell growth while providing the capability of continuous perfusion and simultaneous electrical monitoring using EIS and transistor-based measurements. The first version of this device (Figure $47 \mathrm{a}(\mathrm{i})$ ) consisted of a tubular construct where a PEDOT:PSS scaffold was in situ generated by lyophilization. Cells (MDCK-II or fibroblasts) were grown inside the scaffolds, and the evolution of the cell growth stages was monitored. Figure $47 \mathrm{a}$ (ii) shows the change of the normalized $g_{\mathrm{m}}$ over time. The ability of the device for both inline electrical measurements and perfusion in $3 \mathrm{D}$ cell cultures opened up a new area of integrated bioelectronics toward more physiologically relevant in vitro systems. ${ }^{53}$ Following this work, a more sophisticated version of the previously described tubistor was developed to accurately model the human gut. The so-called "L-tubistor" adopted a hollow lumen structure mimicking the native lumen of the intestine (see Figure $47 \mathrm{~b}(\mathrm{i})$ ). To recreate the gut tissue, fibroblasts, HT29-MTX, and Caco-2 cells (intestinal epithelial cells (IECs), colon adenocarcinoma) were seeded sequentially into the lumen of the scaffold. SEM and confocal images showed the fibroblasts infiltrating the pores of the scaffold, while IECs covering the outer surface of the scaffold expressed a polarized structure, indicative of tissue functionality (Figure 47b(ii, iii). EIS measurements were taken at different time points over 26 days, exhibiting a continuous increase of the impedance magnitude in the mid-to-high frequency range $\left(10^{1}-10^{5} \mathrm{~Hz}\right)$, reflecting tissue formation. (Figure $47 \mathrm{~b}$ (iii, iv)).

3.3.1.3. Fiber Meshes. Fiber meshes bear inherent porosity that facilitates cell attachment and growth. As in any other 3D structure, they can be inherently conducting or they can consist of nonconducting materials and be subsequently coated with a CP coating. The methods used for the development of fibrous meshes can be found in Figure 41. Coating of fiber meshes can 
a
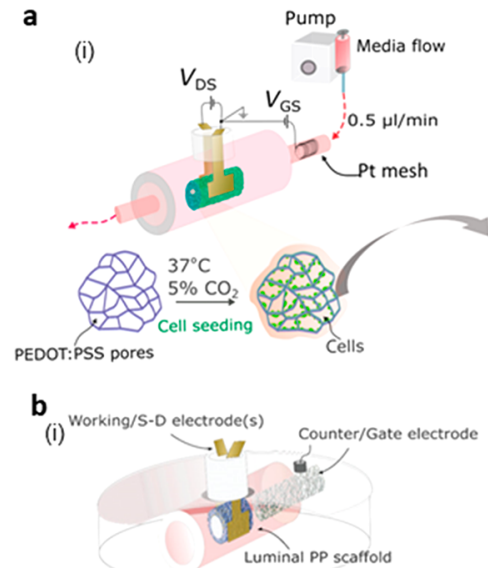
well plate.

(iv)

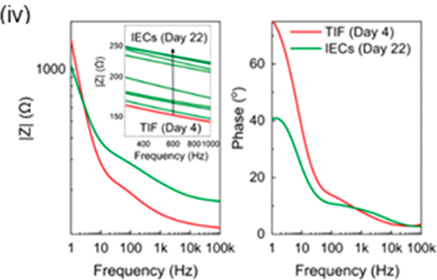

(ii)

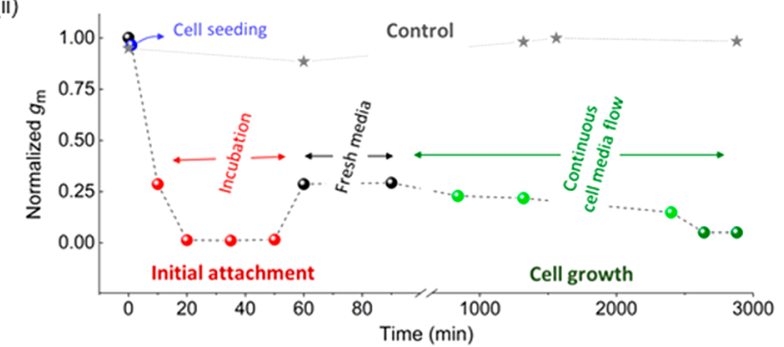

(ii) $1 \quad$ Transverse view
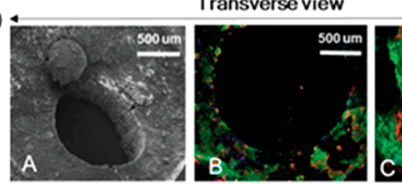
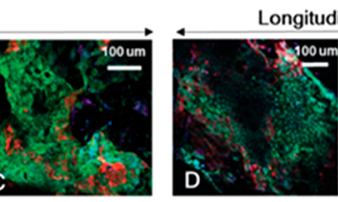

Longitudinal view

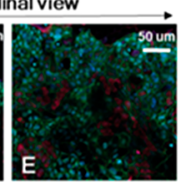

Ortho-view

(iii) PEDOT:PSS scaffold
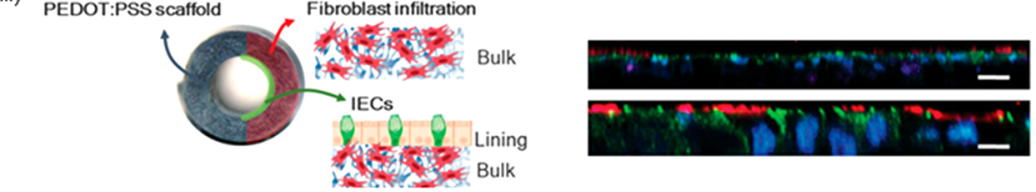

Figure 47. (a) (i) Illustration showing tubistor device used for hosting and monitoring 3D cell cultures under flow conditions. SEM image with MDCK II cells grown for 2 days within PEDOT:PSS scaffold hosted in the tubistor device. (ii) Diagram showing the evolution of normalized transconductance values at different time points during the cell culture duration. Adapted with permission from ref 53. Copyright 2018 AAAS. Licensed under Creative Commons Attribution 4.0 International (CC BY 4.0) License, https://creativecommons.org/licenses/by/4.0/. (b) (i) Schematic of the luminaltubistor (L-tubistor) device used for the development of a 3D bioelectronic human intestine platform. (ii) SEM image showing cultured PEDOT:PSS scaffold with the triculture model (TIF/HT29-MTX/Caco-2). Confocal images showing the corresponding transverse, longitudinal, and orthogonal sections of the cultured L-tubistor. (iii) Schematic illustrating the cellular organization in the scaffold. Orthogonal views (ortho-views, right) of the samples obtained by z-stacked confocal images showing the polarization characteristics of the intestinal tissue. (iv) Bode impedance and phase plots showing the initial response (4 days TIF culture, before IEC) of the 3D electrode and after 22 days of IEC culture. Adapted with permission from ref 40. Copyright 2021 Wiley-VCH Verlag GmbH \& Co. KGaA, Weinheim. Licensed under Creative Commons Attribution 4.0 International (CC BY 4.0) License, https://creativecommons.org/licenses/by/4.0/.

be achieved by chemical polymerization ${ }^{635}$ and VPP, ${ }^{636}$ with the former method being the most common. Bacterial cellulose fiber meshes previously treated with poly(phenylene sulfide) (PPS) have been polymerized with EDOT monomers ${ }^{637}$ while collagen was polymerized with polypyrrole. ${ }^{638}$ In a recent study, Yin and co-workers produced nanofibrous structures of PLA with well distributed PANI nanostructures. PANI structures were realized by dissolving aniline into a PLA/THF solution in the presence of ammonium persulfate (APS). The obtained mixture was transferred to a Teflon mold and frozen at $-80{ }^{\circ} \mathrm{C}$ to complete phase separation. Nanofibrous meshes were obtained after freeze-drying (Figure 48a(i)). Magnified SEM pictures of the nanofibrous structures showed the interconnectivity of the porous structure as well as an ECM-like structure that could be used to promote cell growth (Figure 48a(ii)). Bone marrow mesenchymal stem cells were seeded on the $3 \mathrm{D}$ structures and evaluated by a live-dead (green-red) assay, which confirmed the biocompatibility of the developed structures (Figure 48a(iii)). ${ }^{639}$

Fiber meshes based on CPs can be also realized by electrospinning ${ }^{170,640-642}$ and inflight $3 \mathrm{D}$ printing. ${ }^{643}$ The electrospinning technique consists of the application of an electrostatic field to a polymer solution until a charged liquid ink is formed and printed toward a collector or a surface. During the printing process, the solvent is dried resulting in fiber meshes composed of strains of diameter in the nanoscale. Fiber meshes made from CPs such as PEDOT, ${ }^{413,644,645}$ PANI, ${ }^{460,606,646-650}$ and $\mathrm{PPy},{ }^{170}$ among others, have been successfully realized via electrospinning. Some of them have been mixed with biopolymers to increase their biocompatibility and improve mechanical properties. ${ }^{645,651,652}$ Mixtures of PANI with polycaprolactone have been extensively used in electrospinning for vasculature, ${ }^{653}$ neural, ${ }^{641,654,655}$ and osteogenic differentiation. ${ }^{640,656}$ Stevens and co-workers developed electrospun block copolymers of aniline tetramer and PCL named AT-PCL. Three different concentrations (25\%,50\%, and $75 \%)$ of the ATPCL fiber meshes were developed and the most suitable one was found to be $25 \%$ because of its internal structure and biocompatibility. SEM images taken from the $25 \%$ AT-PCL fiber mesh confirmed its fibrillary structure with diameters of $400 \mathrm{~nm}$ (Figure $48 \mathrm{~b}(\mathrm{i})$ ). The in vitro application of these fiber meshes was evaluated by seeding MC3T3-E1 osteogenic precursor cells. Differentiated cells on phytic acid and pristine membranes were stained for osteocalcin, actin, and nuclei indicating cell differentiation and functionality of the engineered tissue (Figure $48 \mathrm{~b}(\mathrm{ii})){ }^{640}$ Based on the same materials, CasteloFerreira and co-workers developed PCL and PANI fiber meshes by electrospinning. The resulting $3 \mathrm{D}$ structures had a diameter around $373 \mathrm{~nm}$ (Figure 48c(i)). In Figure 48c(ii) a schematic with an illustration of the possible pseudodoping mechanism is displayed. The structures were seeded with neural cells (RENVM) indicating that the PCL-PANI fibers were biocompatible. Finally, the structures were integrated into an electrical stimulation apparatus for cell differentiation. Confocal images from Figure $48 \mathrm{c}$ (iii) of the seeded structures revealed the successful differentiation of REN-VM cells by electrical 
a

(ii)

$\begin{array}{lc}\text { (i) PLA/THF } & \begin{array}{l}\text { In-situ synthesis of } \\ \text { solution }\end{array} \\ \text { PANI nanofibers }\end{array} \quad \begin{gathered}\text { Nanofibrous conductive } \\ \text { composite scaffolds }\end{gathered}$

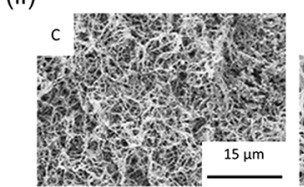

(iii)
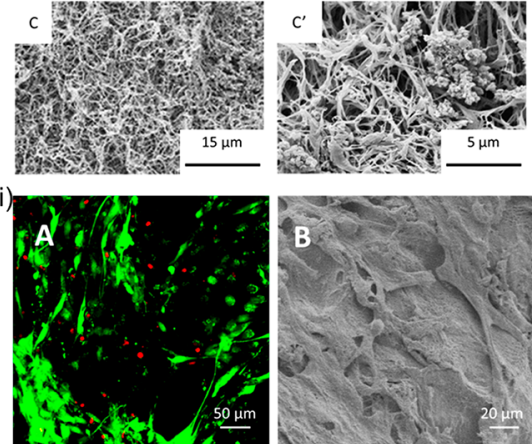

C

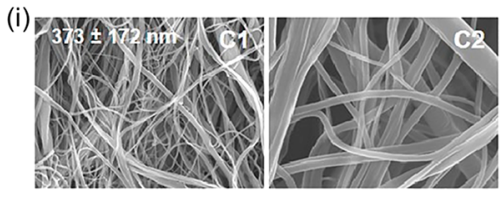

(iii)
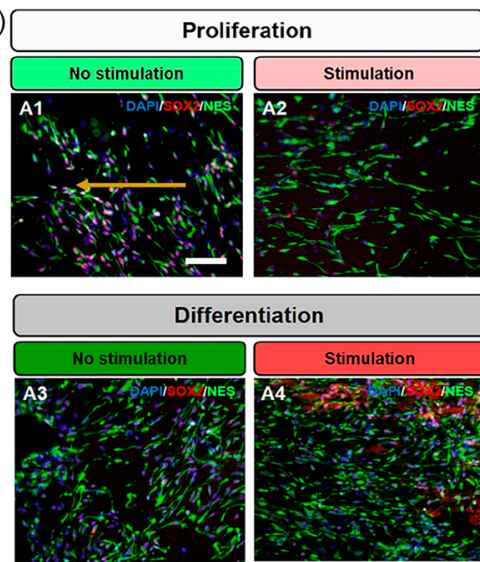

b (i)

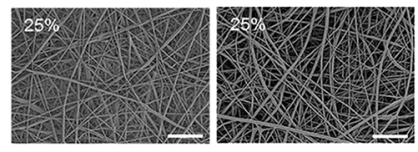

(ii)

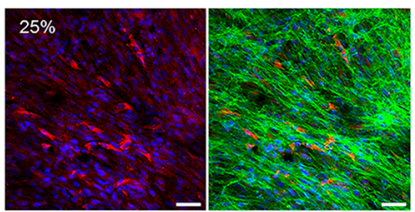

(iii)

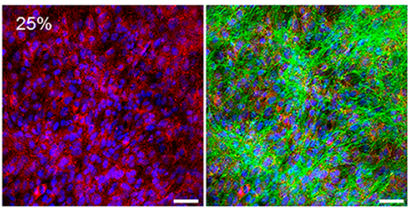

(ii)
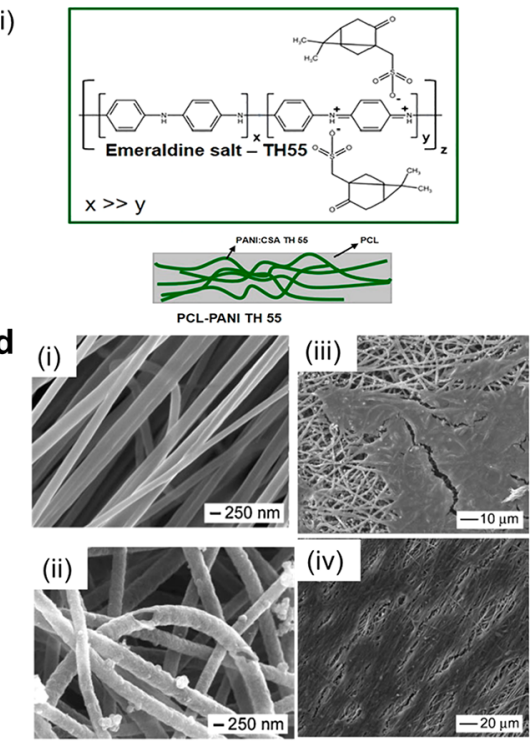

Figure 48. (a) (i) Fabrication process and (ii) SEM images (at different magnification) of the PLA/PANI nanofibrous conducting scaffolds. (iii) Confocal microscopy (live/dead assay) and SEM images showing the morphology of bone marrow-derived mesenchymal stem cells (BMSCs) on scaffold PLA/PANI10 after 1 day of culture. Adapted with permission from ref 639. Copyright 2018 Elsevier. (b) (i) SEM images of conducting electrospun aniline tetramer-co-poly(caprolactone) (here AT-PCL to PCL ratio is $25 \% \mathrm{w} / \mathrm{w}$ ) fibrous scaffolds. Confocal images of cultured MC3T3E1 electrospun AT-PCL (21 days) scaffolds under osteogenic conditions: (ii) phytic acid-doped and (iii) pristine scaffolds. Adapted with permission from ref 640. Copyright 2017 Materials Research Society. Licensed under Creative Commons Attribution 4.0 International (CC BY 4.0) License, https://creativecommons.org/licenses/by/4.0/. (c) (i) SEM images of PCL-PANI electrospun nanofibers produced using a solution of TFE/HFP and a doping agent (camphorsulfonic acid (CSA)) (ii) Mechanism of the pseudodoping effect of PANI/CSA with one of the proposed solvent mixtures used in this work. (iii) Immunofluorescence images showing the proliferation (4 days) and differentiation (4 days) with and without electrical stimulation (bar size $=200 \mu \mathrm{m}$ ). Adapted with permission from ref 641. Copyright 2021 Elsevier. (d) (i, ii) SEM images of PCL nanofibers and PPy nanotubes. The resulting conducting PPy nanotubes were developed by soaking the PCL-PPy core-sheath nanofibers in DCM. SEM images of dorsal root ganglia (DRG) neurite seeded on random (iii) and aligned (iv) core-sheath PCL-PPy nanofiber scaffolds. Adapted with permission from ref 170. Copyright 2020 Wiley-VCH Verlag GmbH \& Co. KGaA, Weinheim.

stimulus. ${ }^{641}$ Templates of PCL have been used to coat PPy nanotubes by in situ polymerization to create a core-sheath structure. Figure $48 \mathrm{~d}(\mathrm{i}$, ii) shows SEM pictures of PCL nanofibers and PPy nanotubes, respectively. The structures were seeded with neurite cells and were assessed by SEM revealing cells aligned onto the PCL-PPy nanofiber structure (Figure $48 \mathrm{~d}$ (iii, iv). ${ }^{170}$

Fiber meshes can be created also by inflight fiber printing. The technique consists of one single step that creates fiber arrays, either suspended or on a surface. Small diameter fibers $(1-3 \mu \mathrm{m}$ thick) can be created by optimizing the fiber sizing. Huang and co-workers developed PEDOT:PSS fibers based on this technique. Mouse fibroblasts were seeded onto $90^{\circ}$ aligned fiber meshes, and confocal pictures corroborated the presence of aligned cells on the fiber mesh, while the histogram showed the orientation of the cell onto the 3D structure (Figure 49a). The aligned fiber meshes were also fabricated on top of a working electrode surface to function as an impedimetric sensor to detect dynamically cell coverage, while simultaneous live-cell imaging was possible given the transparency of the fiber array (Figure 49b). As shown in Figure 49c the impedance magnitude increased in the high-frequency range $(103-105 \mathrm{~Hz})$, most 

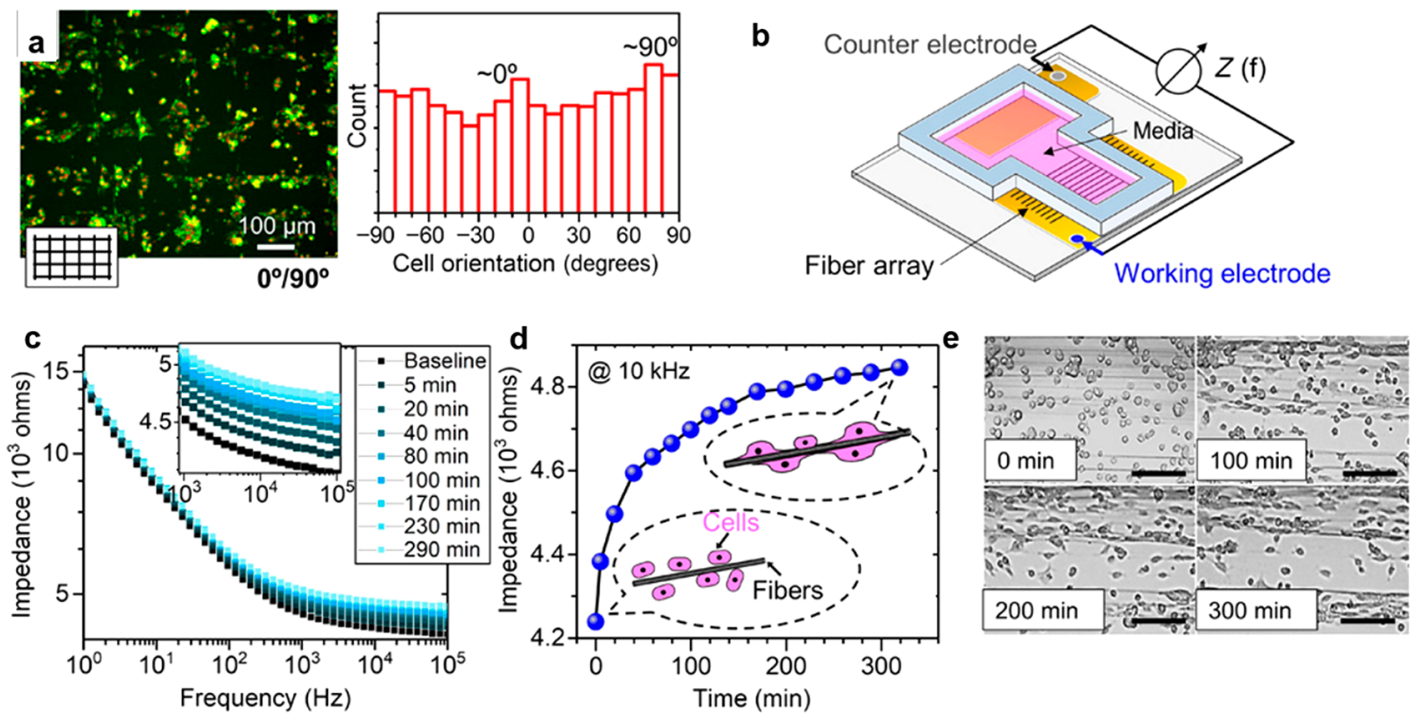

Figure 49. (a) Fluorescence image of $3 \mathrm{~T} 3$ cells (red, nucleus; green, F-actin) grown on 3D fiber arrays fabricated using inflight fiber printing (iFP) technique. Histogram highlights the cellular orientation. (b) Schematic showing the structure of the impedimetric sensor based on iFP. (c) In situ EIS measurements at different time points and $(\mathrm{d})$ the corresponding evolution of impedance values at $10 \mathrm{kHz}$. (e) Microscope images showing the cellfiber interaction for different time points (0,100, 200, and $300 \mathrm{~min}$ ). Adapted with permission from ref 643 . Copyright 2020 the Authors. Published by AAAS. Licensed under a Creative Commons Attribution License 4.0 (CC BY).

a

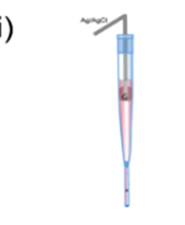

(ii)

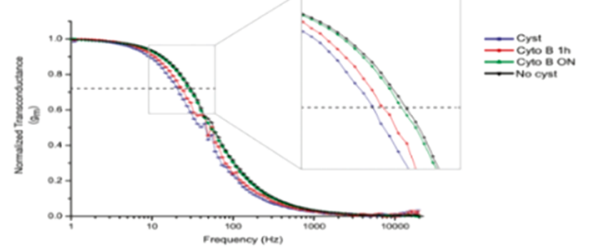

(iii)
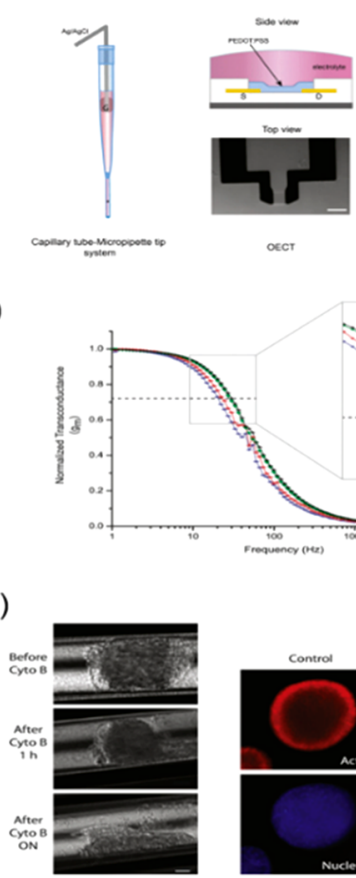

oscr
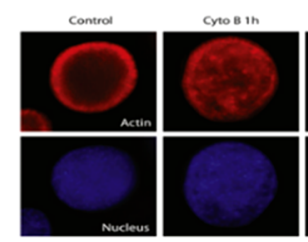

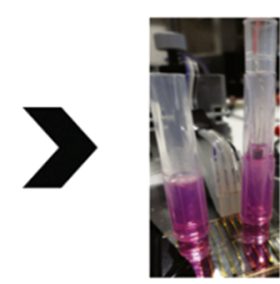

(ii)

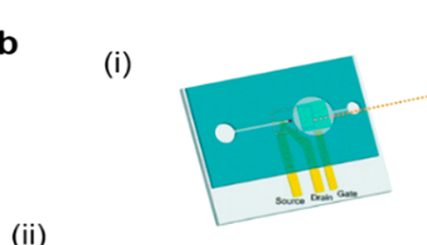

(ii)

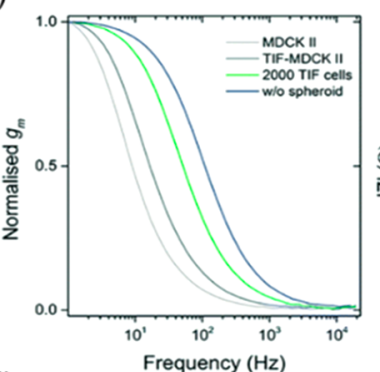

(iii)

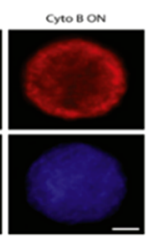

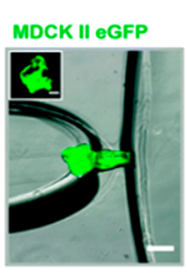
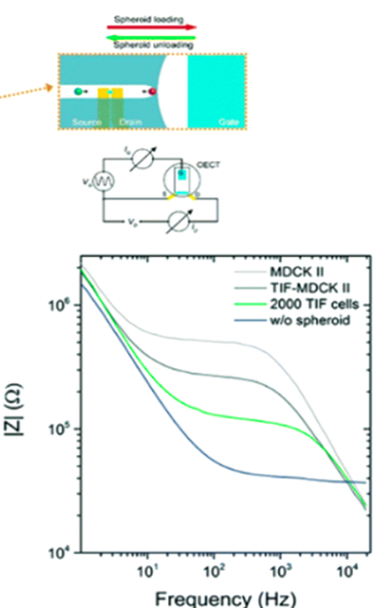

TIF PLifeAct
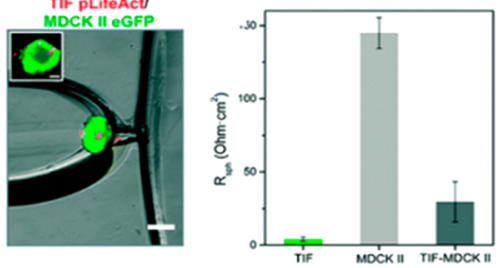

Figure 50. (a) (i) Schematic illustration of an OECT device, combined with a capillary tube-micropipette tip system for the isolation and monitoring of 3D cyst cultures. (ii) Electrical measurements of the toxic effects of cytochalasin B in MDCK I cysts using the OECT device and (iii) brightfield images and confocal images of the MDCK I spheroids inside the capillary tube before and after the treatment with cytochalasin B. Reproduced with permission from ref 659. Copyright 2015 The Authors, published by AIP under a Creative Commons Attribution 3.0 Unported license (CC BY 3.0), https://creativecommons.org/licenses/by/3.0/. (b) (i) Schematic and photograph of the fully assembled spheroid microtrap impedance sensing platform, illustrating the device architecture and working principle for spheroid loading and unloading within the microtrap, and the wiring diagram of the OECT for the cell impedance-based sensing. (ii) Typical frequency dependent response (transconductance) (left) and impedance magnitude spectra (right) of the OECT in the absence and in the presence of different types of spheroids. (iii) Images of MDCK II eGFP (left) and TIF pLifeAct/ MDCK II eGFP co-cultured spheroids (middle) loaded into the circular-shaped nozzle of the microtrap and the respective mean values of the resistance $\left(R_{\text {sph }}\right)$ (right). Reproduced with permission from ref 660. Copyright 2018 Royal Society of Chemistry.

noticeably in the first stages of the cell attachment and fiber-cell alignment. The impedance vs time data at $10 \mathrm{kHz}$ (Figure 49d) show that the evolution of the signal can be correlated to the cellular dynamic data captured by live-imaging in Figure $49 \mathrm{e}^{643}$
3.3.1.4. Toward Organotypic Models with Integrated Bioelectronics. As discussed earlier, in vitro tissue models of higher fidelity are in great demand. The discovery of iPSCs and organoids represents one of the major breakthroughs in the field 
a
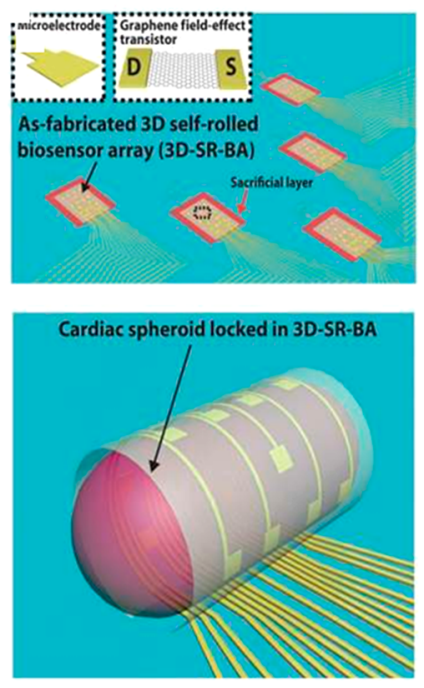
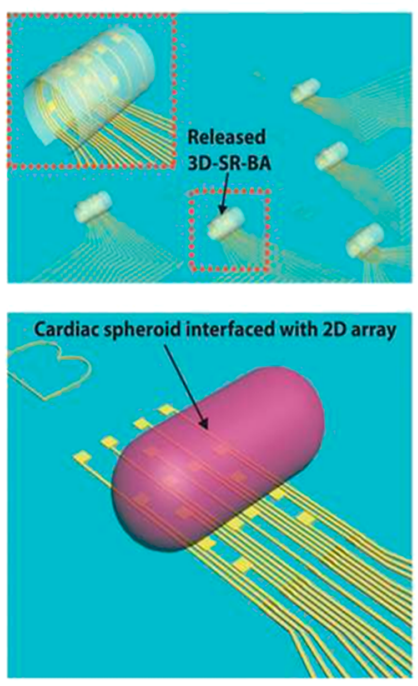

b
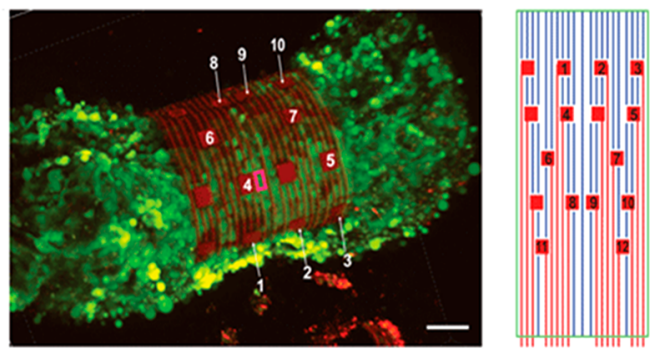

C

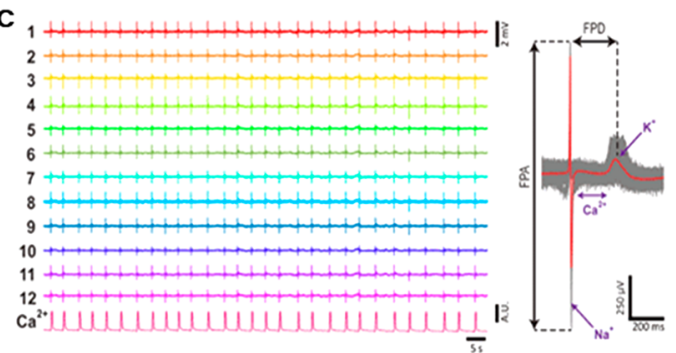

Figure 51. (a) Fabrication process of a 3D self-rolled biosensor array for the encapsulation of cardiac spheroids, (b) 3D confocal microscopy image of the 3D cardiac spheroid and (c) corresponding electrical monitoring of cardiac spheroids. Reproduced with permission from ref 665 . Copyright 2019 The Authors and AAAS under Creative Commons Attribution NonCommercial License 4.0 (CC BY-NC), https://creativecommons.org/licenses/ by-nc/4.0/.

of biomedical research that holds a great promise toward realizing the goal of organotypic models. While this term was originally used to describe cultures of organs or tissues isolated from living organisms, ${ }^{67}$ the advances of $3 \mathrm{D}$ cell-based tools, along with the robustness of the aforementioned cell sources, facilitated the development of 3D organ-like or organotypic culture models that better capture native tissue functional and structural aspects in vitro. ${ }^{658}$ Here, we highlight key examples of such organotypic models integrated with organic bioelectronics, showcasing the great potential of this technology for various applications for in vitro biological science.

One of the early studies of organotypic bioelectronics was performed by our group, involving the establishment of a protocol for the isolation of cyst-like 3D cultures that were then integrated with an OECT for real-time monitoring of the cell status in these 3D structures. More specifically, the OECT was combined with an in-house fabricated capillary tube-micropipette tip system (Figure 50a(i)) that could successfully isolate MDCK-I-derived cysts, precultured within Matrigel, and, by electrically measuring their resistance, assess in real-time the integrity and quality of these cysts. Importantly, this work also demonstrated the possibility to quantitatively predict the toxic effects of drugs on the 3D cell formats by exposing the cysts to cytochalasin B, a compound well-known for its actin cytoskeleton inhibiting activity. These effects were characterized by means of electrical resistance measurements, which reflect the changes in the ion flux through the cysts as a result of the cytochalasin B activity (Figure 50a(ii)). While such effects are commonly evaluated with optical characterization assays (Figure 50a(iii)), this study showcases the added benefits of using OECTs in drug screening and toxicology applications, as they represent a versatile and sensitive tool for both monitoring the quality of $3 \mathrm{D}$ cell formats and generating more predictive data on drug responses and side effects. ${ }^{659}$ This potential of OECTs was further exemplified in a more recent study, in which we developed a novel spheroid microtrap impedance sensing platform (Figure $50 \mathrm{~b}(\mathrm{i})$ ), combining principles from organic bioelectronics, microfluidics, and 3D biology. The performance of the device was validated by testing its ability to measure the resistance of spheroids derived from either barrier-forming cells or non-barrier-forming cells and their cocultures. Similar to the previous study, here it was also found that the permeability of ions in each spheroid type can be co-related with the differences in the resistance. As expected, spheroids derived from barrierforming cells (i.e., MDCK-I epithelial cells) induced a dramatic decrease in the cut-off frequency and impedance magnitude, compared to spheroids derived from non-barrier-forming cells (i.e., fibroblasts) (Figure 50a(ii)). This is also reflected in the resistance profile of each type of spheroid (Figure 50a(iii)), indicating that this sensing platform can discriminate between different types of spheroids according to their ion permeability profiles (which depend on the nature of the cells from which they are formed). In addition, the impedance sensor was tested for its ability to monitor the effects of toxic compounds on the cell membrane integrity, showing great promise as a tool for toxicology measurements. ${ }^{660}$ Finally, we also investigated the potential to integrate neurospheres with patterned PEDOT:PSS MEAs and present an improved method to control the location of neurospheres on the device and to establish better cellelectrode coupling for enhancing electrophysiology recordings. ${ }^{223}$

Over the last couple of decades, the field of 3D cell biology has witnessed major breakthroughs, which have significantly advanced our ability to recreate more physiologically relevant in vitro models in the lab, highly valuable for all sorts of biomedical applications. Advances in stem cell research and, in particular, iPSCs paved the way for the discovery of organoids or in other words "organs in a dish" or organotypic cultures. ${ }^{661-663}$ Although the term has often used to describe spheroid cultures or generally in vitro models of tissues that resemble organs, nowadays, a 3D cell structure, to be defined as an organoid, should fulfill specific criteria: to contain multiple cell types and to exhibit some aspects of the native organ self-organization patterns and specialized functionality, thus retaining the identity of its in vivo counterpart. ${ }^{661}$ As such, organoids represent a more accurate imitation of the real organ ${ }^{25}$ and thus a versatile tool for 
a scrutiny of applications, ranging from fundamental cell biology to tissue engineering and personalized medicine. ${ }^{16,663,664}$ Despite the great progress of the technologies used in organoid research, there is still a gap in properly monitoring and characterization of such complex, multicellular systems. Taking advantage of the ability of organic electronics for highly sensitive, noninvasive, and real-time monitoring of complex tissues, Kalmykov et al. ${ }^{665}$ recently presented the "organ-on-echip”, a novel tool for electrophysiological measurements of cardiac spheroids, opening the way for implementation of this technology in organoid research. The organ-on-e-chip is a PEDOT:PSS-based 3D self-rolled biosensor array, interfaced with human stem cell-derived cardiac spheroids (Figure 51a,b), that can be operated either as a microelectrode platform for EIS monitoring or as a FET for continuous multiplex electrophysiological recordings of the $3 \mathrm{D}$ tissue with superior spatiotemporal resolution (Figure 51c). In fact, the device was shown to be able to closely follow the propagation of electrical information through the 3D multicellular structure with high spatiotemporal resolution that outperforms commonly used assays (e.g., calcium transient imaging). Tools with such high biosensing capabilities are in high demand since they can help to elucidate the complex interactions between cells in 3D formats, both in homeostatic and in pathological conditions, as well as to assist drug development studies. ${ }^{665^{\circ}}$

\subsection{Stimulation Applications}

The role of bioelectricity in living systems has been highlighted by numerous researchers throughout time. The first to bring this aspect to the attention of the wider community was Luigi Galvani with his work done in the late 18th century on frog muscles. ${ }^{666}$ Bioelectricity and its importance during embryogenesis and tissue regeneration have been supported and demonstrated by different authors. ${ }^{667,668}$ The work done by Michael Levin's team has investigated the influence of membrane potential on stem cell differentiation and how, by altering it, it is possible to modulate or hinder a stem cell's differentiation potential. ${ }^{669,670}$ Therefore, the possibility to affect cell behavior by addressing cell membrane polarity has been leveraged to develop strategies for tissue engineering approaches. Several studies have shown the efficacy of different stimulation approaches in inducing cell migration and proliferation as well as directing stem cell differentiation. ${ }^{671-674}$

Since CPs have entered the field of biomedical research, electrical stimulation protocols have been explored in combination with conducting substrates. The reasons for the combined use of physical stimulation and electrically active materials are multiple. The electroactive substrates allow the use of milder stimulation conditions, they can deliver the stimulation with no need for the electrodes to be in direct contact with the cells or the culture medium, and they can deliver charged molecules functioning as drug delivery devices. Recent reviews have highlighted the potential of the combined use of electrical stimulation protocols and conducting materials and stem cells relevant for tissue engineering applications in both in vitro and in vivo studies. ${ }^{675-677}$ In this section, we report some key studies showcasing the use of conducting polymers in tissue engineering approaches, for stem cell-directed differentiation, as well as neuronal and cardiac cell modulation, through a variety of bioelectronic devices and methodologies.

3.4.1. Stimulation of Electrogenic Cells. Electrogenic cells, in addition to producing electrical signals, will often also respond to electrical stimulation. This concept is perhaps most famously evidenced in cochlear implants and pacemakers, where electrical pulses are used to dictate neural firing and cardiomyocyte contraction, respectively. Similarly, organic electronic devices and materials can be constructed for in vitro use to elicit similar behaviors in electrogenic cells. However, stimulation has a variety of meanings, even in the context of electrogenic cells, spanning from induced neural firing to altered phenotypes and cell behaviors. Here, we will discuss these applications in the context of in vitro electrogenic cell cultures, specifically focusing on neurons and cardiomyocytes.

One of the most commonly applied in vitro methods of electrical stimulation for electrogenic cells is application to adhered cells through an electroactive substrate. An early study in this field, performed by Schmidt et al., involved the culture of PC-12 cells on a PPy-based substrate. ${ }^{152}$ During culture, the researchers applied a constant voltage of $100 \mathrm{mV}$ between the substrate and a gold counter electrode for $2 \mathrm{~h}$, finding longer neurites in PC-12 cells under electrical stimulation. ${ }^{152}$ Since then, a host of studies examining PC-12 neurite outgrowth under constant applied voltage or current have been performed, showing consistently longer neurites from PC-12 cells under electrical stimulation for PEDOT-based, ${ }^{678} \mathrm{PPy}-$ based, ${ }^{161,679-684}$ and PANI-based ${ }^{685}$ substrates. Some of these studies have also examined the resulting PC-12 cultures for neuronal markers, finding that these markers are upregulated under constant voltage electrical stimulation. ${ }^{686-688}$ These data indicate that electrical stimulation can induce differentiation of PC-12 cells into neuronal cells. Other in vitro studies on primary cells have also shown promising results. Heo et al. found that application of an electrical stimulus to a dorsal root ganglia (DRG) cell line embedded in gelatin methacryloyl (GelMA) through a surrounding PEDOT:PSS-based grid showed enhanced expression of a variety of neuronal markers versus no stimulation and stimulation without the PEDOT:PSS grid. ${ }^{689}$ Rajnicek et al. also showed that primary spinal neurons cultured on PEDOT-based substrates will extend neurites toward the cathode under an electric field applied through the medium surrounding the substrate. ${ }^{690}$

Other studies have examined different stimulus regimens, specifically monophasic pulses and biphasic pulses. Similar to constant voltage, monophasic stimulation generally shows improved neurite outgrowth in PC-12 cells, ${ }^{691,692}$ with some studies also showing increased expression of neuronal markers. ${ }^{63}$ Other studies have also applied this monophasic regimen to SH-SY5Y cells, ${ }^{694}$ in particular showing a response measured through calcium imaging that decreased upon subsequent pulses. ${ }^{636}$ One study applied both constant current and monophasic pulse stimuli to PC-12 cells on PPy substrates, finding that the number of cells showing neurites and the number of neurites on these cells were highest for monophasic pulses, then constant current, followed by no stimulation. However, the neurite length was similar for both monophasic and constant current stimulation, both of which were enhanced versus no stimulation. ${ }^{65}$ Biphasic pulse regimens also show improved neurite outgrowth for PC-12 cells ${ }^{182,202,696}$ and enhanced neuronal marker expression for PC-12 ${ }^{697}$ and $\mathrm{SH}$ SY5Y cells. ${ }^{698}$ Interestingly, Zhu et al., in addition to showing longer neurite outgrowth from PC-12 cells, also found that biphasic electrical stimulation increased the excretion of nerve growth factor (NGF) from Schwann cells versus no stimulation. ${ }^{154}$ Other studies examining biphasic stimuli to primary cultures found lengthening of cells from retinal ganglia on PPy films, ${ }^{69}$ increased neurite outgrowth from cortical 
neurons on PPy substrates, ${ }^{700}$ and increased neurite length in DRG explants. ${ }^{701}$ Nguyen et al. examined the outgrowth of axons from DRG explants under constant voltage and biphasic stimuli in a nerve conduit setup, finding that these explants grew longer axons under biphasic stimulation versus constant voltage and no stimulation. ${ }^{702}$ Generally, electrical stimulation through an electroactive substrate appears to enhance neuron-like properties in cells, regardless of stimulation regimen, but more temporal control over the applied stimulus seems to promote better neuron-like phenotypes. However, more research will need to be performed on these types of stimulation regimes to confirm these phenomena.

Organic devices can also be designed to initiate firing in neuronal cells. This application is of particular interest given the possibility of neural stimulation in vivo. ${ }^{484}$ The most prominent of devices within the area of neural stimulation is the stimulating electrode. These electrodes must be designed to provide a sufficient electric field to stimulate cells without damaging them. ${ }^{703}$ Given the extrinsic nature of these experiments, some studies have utilized single electrodes for stimulation, ${ }^{704}$ but these devices are still usually constructed in an array architecture. $^{120,487,705-710}$ Stimulation through electrodes still results in neurite outgrowth, ${ }^{706}$ even when applied in $3 \mathrm{D} .^{710}$ However, neuronal cells can also be induced to show ionic changes when electrically stimulated. ${ }^{704,707}$ MEAs can also be constructed to both stimulate and then record the result of the electrical stimulus, ${ }^{120,705,708,709}$ as reported in the work from Aqrawe et al. (Figure 52). ${ }^{487}$ An early study in this area utilized an array of ITO electrodes with PEDOT:PSS coatings. This study found that rat cortical neurons show spiking behavior when electrically stimulated with a biphasic pulse. ${ }^{705}$ Nikiforidis et al. fabricated a PEDOT-based MEA for culturing cortical neurons (Figure 52a). ${ }^{120}$ In this study, simultaneous traces of neural firing were recorded with calcium imaging after a biphasic pulsed stimulus was applied through the MEA (Figure 52b-e). These data are quite complex, given that a single electrode can record spikes from multiple surrounding neurons, thus highlighting the difficulty of these types of experiments. However, the successful evocation of spiking behavior from these cultured neurons is evident (Figure 52d,e). ${ }^{120}$

Various other devices have been utilized for electrically stimulating neurons. Benfenati et al. showed spiking behavior, recorded using a patch-clamp setup, that was associated with a monotonically increasing pulse of the gate-source voltage from a P13-based transistor setup. They were also able to record electrophysiologically derived signals with their device. ${ }^{56}$ Other devices have focused on the electrical initiation of drug delivery to neurons. Isaksson et al. originally reported the production of a PEDOT:PSS-based ion pump for neuronal cells. ${ }^{315}$ Tybrandt et al. utilized a similar device to deliver acetylcholine to SH-SY5Y cells (Figure 53a). ${ }^{310}$ This OEIP functions by applying a bias between two PEDOT:PSS electrodes, where the positive terminal becomes oxidized and the negative terminal reduced, thus generating an electric field and pushing cations through a channel from the source electrode to the target electrode, as described in section 2.2.3 (Figure 53a,b). This device was used to move acetylcholine, a positively charged neurotransmitter. The waste electrode assisted in prefilling the channels to improve delivery accuracy. Upon application of a voltage between the source and target electrodes, calcium imaging revealed a response in SH-SYSY cells near the channel opening. As subsequent voltage pulses were applied, this response increased and eventually reached cells farther from the channel a
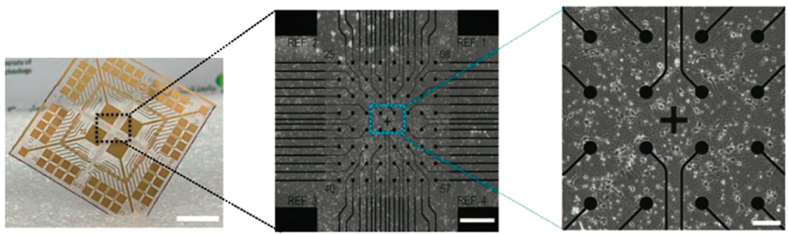

b Stimulation OFF

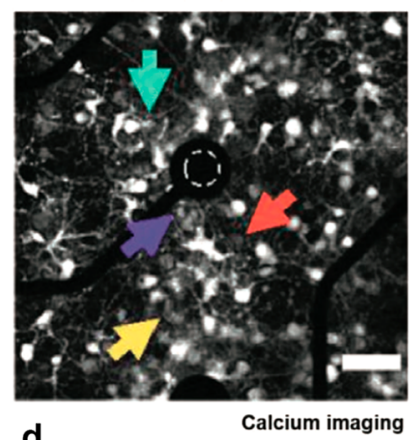

d

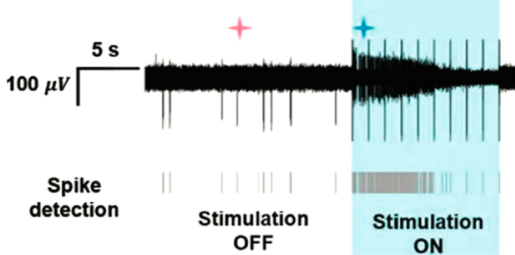

C Stimulation ON

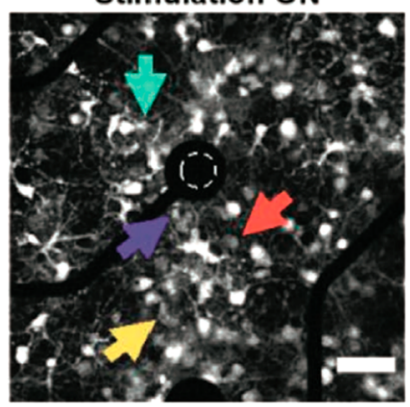

e

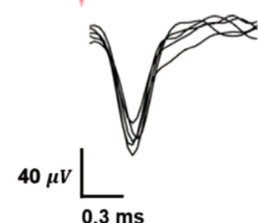

$0.3 \mathrm{~ms}$

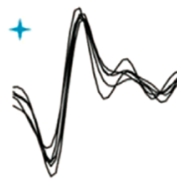

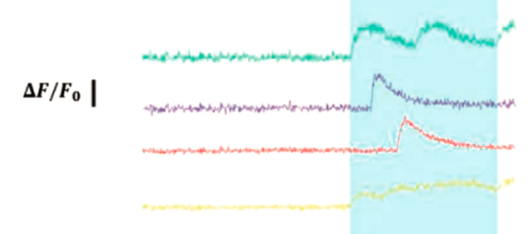

Figure 52. Recording and stimulation of mouse cortical neurons on $\mathrm{P}\left(\mathrm{EDOT}\right.$-ran-EDOTOH): $\mathrm{CLO}_{4} / \mathrm{EG}$ MEA. (a) Image of MEA device with insets showing neuron cultures on device after 11 days. Scale bars are $1 \mathrm{~cm}, 500 \mu \mathrm{m}$, and $100 \mu \mathrm{m}$, from left to right. Fluorescent images showing neurons (b) with no applied stimulus and (c) with stimulus (10 biphasic current pulses) applied. Fluorescence is derived from $\mathrm{Ca}^{2+}$ imaging. Arrows correspond to fluorescence traces in panel d, and white circle indicates recording electrode for panel d. Fluorescence traces from $\mathrm{Ca}^{2+}$ imaging (top) and corresponding voltage recordings (bottom) of neurons with stimulation off and on. (e) Collapsed waveforms of traces with simulation off (top) and on (bottom). Adapted with permission from ref 120 . Copyright 2020 Wiley-VCH $\mathrm{GmbH}$.

opening (Figure 53c). ${ }^{310}$ Further iteration on these types of devices has since resulted in a device that can both sense and apply neurotransmitters like acetylcholine. ${ }^{711}$ Other drugreleasing devices include a PPy-based substrate with cultured SH-SY5Y cells ${ }^{712}$ and the previously mentioned cardiac patches from Feiner et al., in which drugs are released from PPy electrodes to stimulate cardiac cells. ${ }^{504,505}$

Another major area of work within this field is the photostimulation of neurons. One relatively straightforward application of organic electronics in photostimulation is the usage of OLEDs for optogenetic modulation of cells. Optogenetics is the induction of light-sensitive molecules in cells to allow for modulation of the cell's activity. ${ }^{713}$ As such, OLEDs have been used to optogenetically control different neuronal populations. ${ }^{714-716}$ Another promising area of work for neuronal modulation is the use of organic optoelectronic materials. Different studies have shown the propensity to initiate 

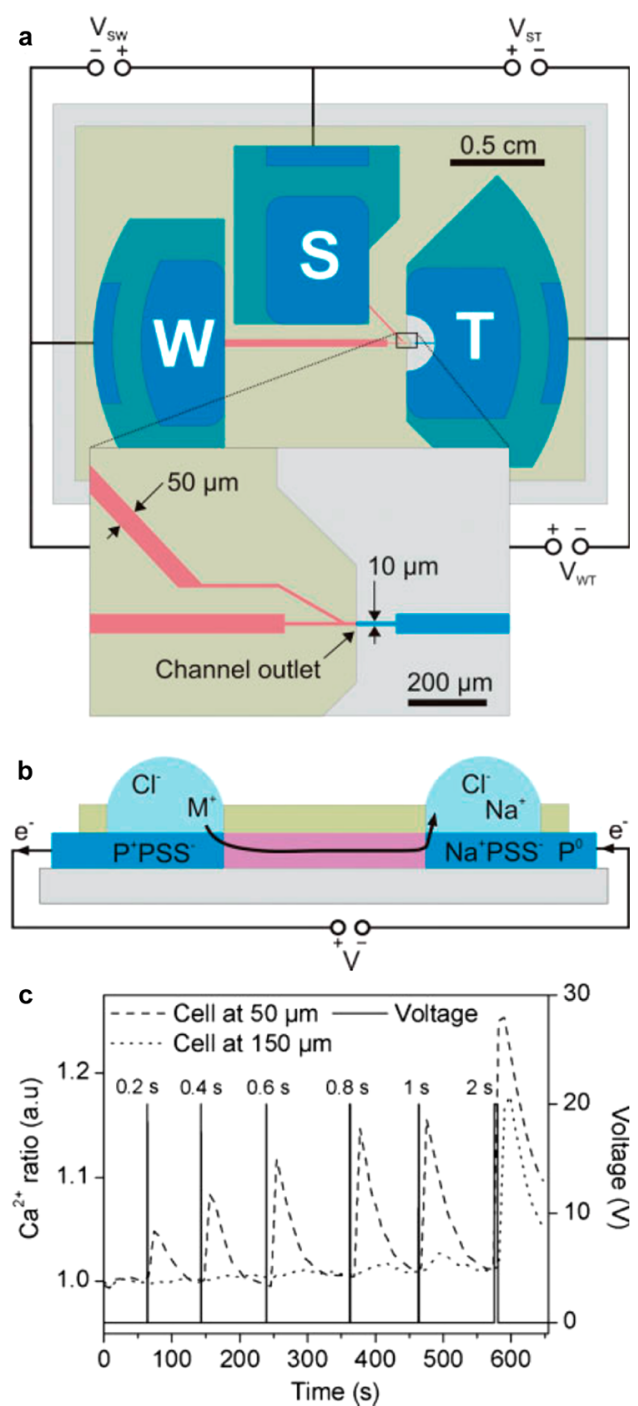

Figure 53. Ion pump for application of acetylcholine to SH-SY5Y neuronal-like cells. (a) Schematic showing design of ion pump device. Source $(\mathrm{S})$, target $(\mathrm{T})$, and waste $(\mathrm{W})$ regions are connected to ionically drive acetylcholine from the source to the target, thus providing drug-induced stimulus for cells. Inset shows fluidic connector channels between regions. (b) Cross-section showing device function. Application of a voltage drives a positively charged molecule, that is, acetylcholine, through the interconnecting channel. (c) Time trace showing applied source-target voltage and resultant $\mathrm{Ca}^{2+}$ fluorescence ratio in cells in target channel as acetylcholine reaches cells $<50 \mu \mathrm{m}$ and $>150 \mu \mathrm{m}$ from channel opening. Adapted with permission from ref 310 . Copyright 2009 Wiley-VCH Verlag GmbH \& Co. KGaA, Weinheim.

neural firing through optoelectronic photostimulation $^{134,318,322,328,717-719}$ or even silencing. ${ }^{720}$ These devices have also been shown to induce neurite outgrowth in PC-12 cells, as reported beforehand. ${ }^{721,722}$ The operating mechanism of these devices for neural stimulation can vary somewhat between initiated photocurrents, photothermal effects, and photocapacitive effects depending on the device and the desired application. Jakešová et al. demonstrated a photocapacitive device for modulation of membrane potential in a large oocyte cell expressing voltage-gated ion channels (Figure 54). ${ }^{329}$ This study shows the fabrication of a $\mathrm{p}-\mathrm{n}$ junction, constructed from the p-type semiconductor, metal-free phthalocyanine $\left(\mathrm{H}_{2} \mathrm{Pc}\right)$, and the n-type semiconductor, $N, N^{\prime}$-dimenthylperylene3,4:9,10-tetracarboxylic diimide (PTCDI). This device acts as a photocapacitor under light pulses, for capacitive coupling with an adjacent cell (Figure 54a). The transient voltage in the oocytes was found to match closely with modeled data, indicating that the membrane potential of the oocyte can be modulated with the photocapacitor (Figure 54b). Stimulation experiments of the oocyte show that without voltage-gated potassium channels (Shaker $\mathrm{K}_{\mathrm{v}}$ channel) change in membrane potential and subsequent photostimulation do not alter the outward current from the cell (Figure 54c). However, with the presence of these channels, the outward current can be increased over the clamped current using a pulsed photostimulation (Figure 54d). These data show that the oocyte's voltage-gated channels can be precisely modulated through photostimulation. $^{329}$

Numerous studies have also examined the application of electrical stimulation to cardiac cells, typically through electroactive substrates. Some of these studies have applied monophasic pulsed stimuli to cardiac cell cultures. ${ }^{723-725}$ Application of this type of electrical stimulus to cardiomyocytes appears to affect the frequency of firing behavior. In an early study in this area, Nishizawa et al. found that an applied current pulse results in spiking that can be observed through calcium imaging. ${ }^{726}$ Hsiao et al. also found that cardiomyocyte firing frequency can be altered and firing rate can be synchronized through application of an electrical stimulus. ${ }^{727} \mathrm{Kim}$ et al. also showed that cardiomyocyte beating frequency can be modulated through application of biphasic pulses through a PEDOT:PSS substrate (Figure 55). ${ }^{493}$ This study utilized a $2 \mathrm{~ms}$ pulse at $0.8 \mathrm{~V}$ followed by $2 \mathrm{~ms}$ pulse at $-0.8 \mathrm{~V}$ with a subsequent rest period out to $0.5 \mathrm{~s}$ (Figure 55a). This regimen elicited an increase in the beating frequency of primary rat cardiomyocytes, as measured by calcium imaging (Figure 55b). ${ }^{493}$ Other stimulus application modalities should also be examined; for example, Thourson et al. also applied biphasic pulses to cardiac cells through PEDOT:PSS microwires. ${ }^{728}$

Some organic culture systems have focused on electromechanical stimulation, primarily through piezoelectric materials. Poly(vinylidene fluoride) (PVDF) is a piezoelectric polymer and has been used to stimulate neurite outgrowth in PC-12 cells through application of ultrasound. ${ }^{729}$ It has also been used with $\mathrm{DRG}^{730}$ and spinal cord-derived neurons. ${ }^{731}$ Organic piezoelectrics have also been applied to cardiac cells, ${ }^{732,733}$ including for the production of biogenerators derived from the combination of the spontaneous contraction of cardiac cells and the piezoelectric nature of PVDF. ${ }^{734}$ The relevance of organic piezoelectric materials to electrogenic cells has not been fully explored, especially with the theoretically ability of piezoelectrics to convert cardiomyocyte contraction to a measurable potential. Other reviews have examined this question in detail. $^{735}$

3.4.2. Stem Cells. Stem cell research has laid the foundation for personalized cell-based therapies of major diseases, while their ability to differentiate into other types of cells revealed great potential for regenerative medicine. In parallel, advancements in tissue engineering technology, particularly the use of polymeric biomaterials, ${ }^{736}$ made possible the in vitro remodeling of stem cells grown into $3 \mathrm{D}$ tissue. ${ }^{737}$ Externally applied electrical stimulation has been shown to affect important cell functions involved in regeneration by modulating the local membrane potential $\left(V_{\text {mem }}\right)$. The inherent soft mechanical properties, and mixed conduction properties of CPs enabled the realization of highly biomimetic, electroactive interfaces to study 

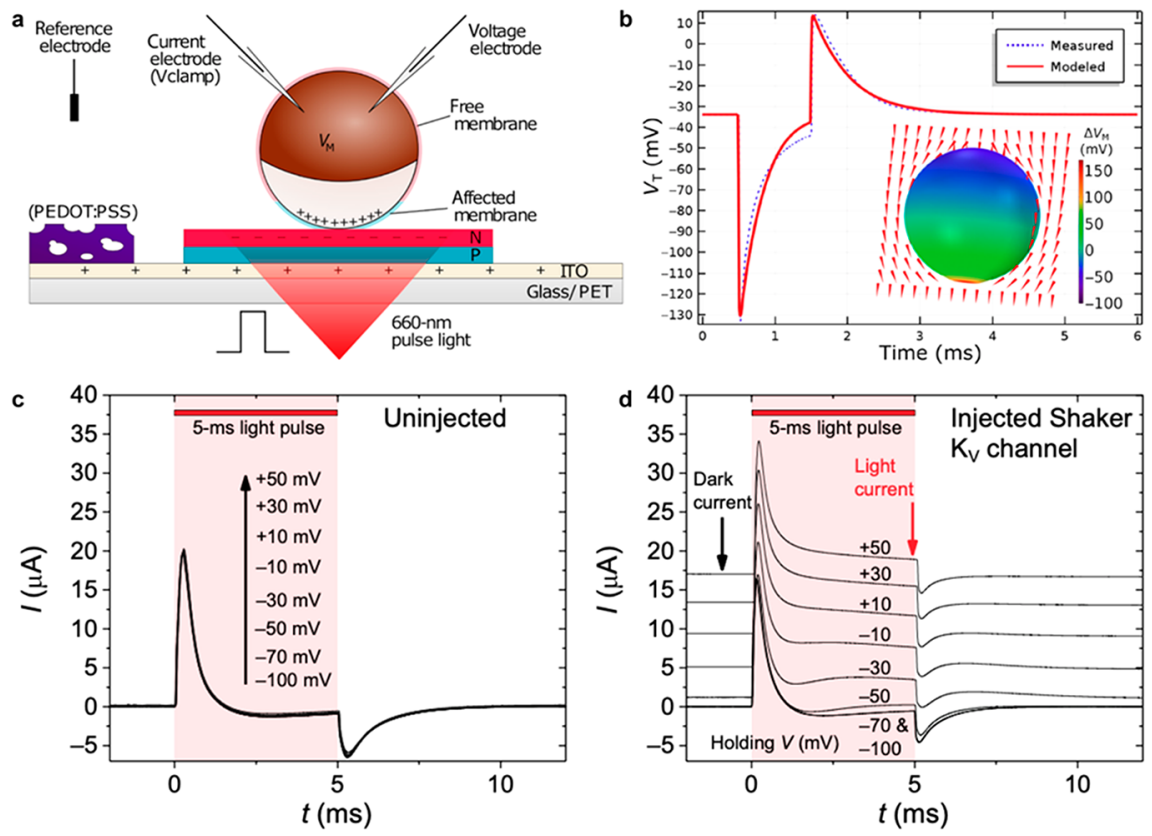

Figure 54. Photocapacitive device for modulating electrogenic cells. (a) Schematic of device layout. Input light induces capacitive coupling between device and cell through pn junction. Cell is simultaneously monitored using patch-clamp setup. (b) Plot showing transient potential $\left(V_{\mathrm{T}}\right)$ as a function of time for a model of the cell over the course of light pulse-based photostimulation compared to measured data. Model indicates that membrane potential $\left(V_{\mathrm{M}}\right)$ changes due to capacitive coupling of the device and the cell. (c, d) Plots showing outward current from a cell through voltage-clamp measurements without and with voltage-gated ion channels (Shaker $\mathrm{K}_{\mathrm{V}}$ channel), respectively, under photostimulation. Adapted with permission from ref 329. Copyright 2019 Jakešová et al. Published by AAAS and licensed under Creative Commons Attribution 4.0 International License, https:// creativecommons.org/licenses/by/4.0/.

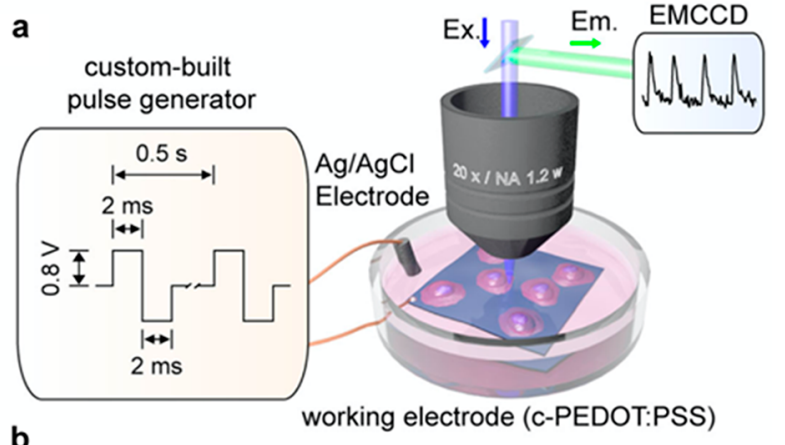

b

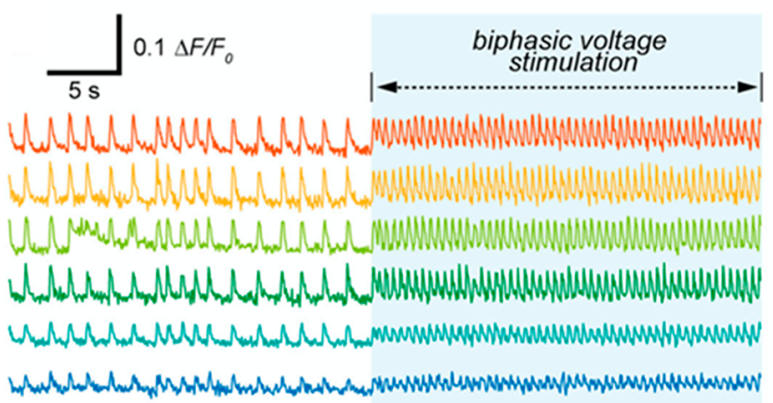

Figure 55. Electrical stimulus applied for modulation of beating frequencies of neonatal rat cardiomyocytes. (a) Schematic of layout for application of biphasic pulsed stimulus. (b) $\mathrm{Ca}^{2+}$ fluorescent recordings of 6 different cells before and after application of stimulus. Adapted with permission from ref 493. Copyright $2018 \mathrm{Kim}$ et al. Published by Springer Nature and licensed under Creative Commons Attribution 4.0 International License, https://creativecommons.org/licenses/by/4.0/.

the effects of ES on stem cell proliferation and differentiation in vitro.
3.4.2.1. Neural Stem Cells. Adult human NSCs are able to differentiate to all neural lineages in the brain, and therefore, NSC-based treatments are promising to treat brain injuries and neurological disorders. They are also excellent candidates for in vitro modeling of neural development and fundamental cell function studies. A recent book chapter from TomaskovicCrook et al., ${ }^{738}$ evidenced the great impact of electrical stimulation on NSC cell fate and function in vitro. ${ }^{648}$ Despite the general understanding of the effect of ES on the cell membrane potential, ${ }^{739}$ the exact cellular mechanisms that governed NSC differentiation upon ES are yet not well understood.

CP-based interfaces have been used to study the effects of ES on the NSC proliferation and differentiation in vitro. In an interesting work by Tomaskovic-Crook et al., NSCs were derived from human midbrain and encapsulated within a conducting polysaccharide-based biogel attached to PEDOT:PSS 3D pillar MEAs. ${ }^{225}$ These individually addressable pillars were used to stimulate NSCs in situ during differentiation and promoted cell adhesion, migration, and neurite outgrowth. Furthermore, Stewart et al. described the effects of electrical stimulation on the differentiation of multipotent human NSCs, cultured on top of thin films of polypyrrole (PPy) doped with dodecylbenzenesulfonate (DBS). Electrically stimulated hNSC cultures show an abundance of Tuj1-expressing neurons, and they exhibited a greater number of neurites and increased neurite branching and length compared to unstimulated hNSC cultures on PPy:DBS thin films. ${ }^{740}$ Ritzau-Reid et al. developed a conducting scaffold based on EDOT-poly(caprolactone), a block copolymer, via melt electrospinning writing and seeded it with iPSC-derived NSCs (Figure 56a). Immunofluorescence measurements proved that the proposed copolymer was cytocompatible with excellent mechanical properties suitable 
a

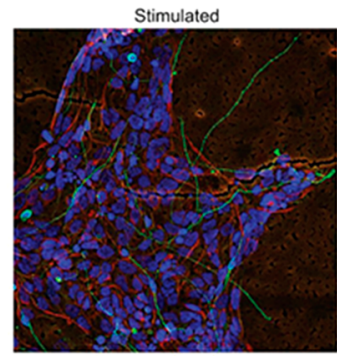

b

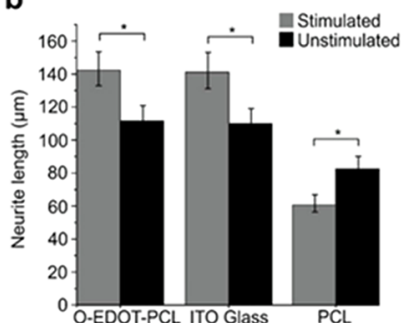

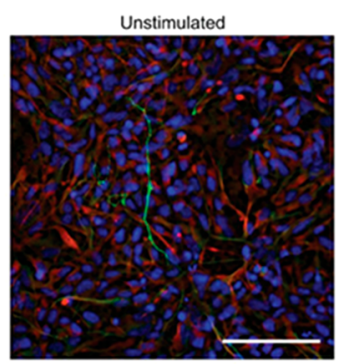

C

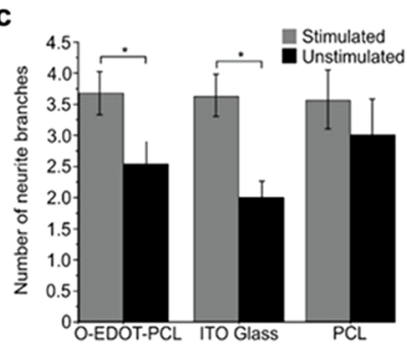

Figure 56. (a) Fluorescence images of stimulated (left panel) and unstimulated (right panel) NSCs cultured on oligo(EDOT)-PCL films ( $\beta$ III-tubulin, green; nestin, red; DAPI, blue). Scale bar $100 \mu \mathrm{m}$. (b) Mean neurite length and (c) neurite branching of NSCs with or without electrical stimulation. Adapted with permission from ref 741 . Copyright 2020 The Authors. Published by John Wiley and Sons, licensed under the terms of the Creative Commons CC BY license, https://creativecommons.org/licenses/.

for stem cell culture. Upon ES, the neurite length (Figure 56b) and branching (Figure 56c) of neural stem cells was enhanced in the materials under electrical stimulation, demonstrating the potential of these scaffolds for neural tissue engineering. ${ }^{741}$ Similar results were also shown using an electrically conductive PPy:hydroxyapatite (HA) hydrogel scaffold to accelerate the differentiation of NSCs with electrical stimulation. ${ }^{99}$

Another study from Sordini et al. evaluated the effect of electrical stimulation on ReNcell-VM, a neural progenitor cell

line with the ability to readily differentiate into neurons and glial cells, cultured on PEDOT:PSS. ${ }^{162}$ Both immonufluorescence and polymerase chain reaction show that NSC differentiation into neurons is enhanced with pulsed DC electrical stimulation (Figure 57). The study also investigated the compatibility of PEDOT:PSS cross-linked with both GOPS and DVS as well the influence of different electrical stimulation conditions (AC, DC, and pulsed DC electrical fields).

Interestingly, $\mathrm{Heo}$ et al. demonstrated the potential of adipose-derived stem cells (ADSCs) to differentiate into neural precursor cells with the assistance of ES using CPs. ${ }^{742}$ They used an array of microwells integrated with a conducting hydrogel made of poly(ethylene glycol) (PEG) diacrylate and PEDOT:PSS with good mechanical and electrical properties. ES was applied with the assistance of two electrodes placed on both ends of each of the well plates and $1 \mathrm{~V}$ was applied continuously for 10 days. Neuronal gene expression greatly increased when cells were treated with ES. In addition, ADSC aggregates with ES were more interconnected with a higher number of neurites than untreated groups. The examples we reported in this section clearly show the potential of the combined use of CPs and electrical stimulation in directing targeted processes for both basic research and targeted regenerative approaches.

3.4.2.2. Toward Bone Forming Cells. In the late 1950s, the piezoelectricity of bone was demonstrated. ${ }^{743}$ Electric fields are indeed generated within the bone in response to mechanical stimulation, and electrical stimulation generates mechanical deformation within the bone. The generated negative charges can open voltage-gated calcium channels on osteocytes, in turn triggering cascades of signaling pathways leading to osteoblast activation, proliferation, and differentiation, extracellular matrix deposition, and subsequent bone formation. Inspired by bone piezoelectricity, the use of CPs for bone tissue engineering has been investigated to develop both in vitro $3 \mathrm{D}$ models and targeted regenerative approaches. ${ }^{52,744}$ Other than neural stem
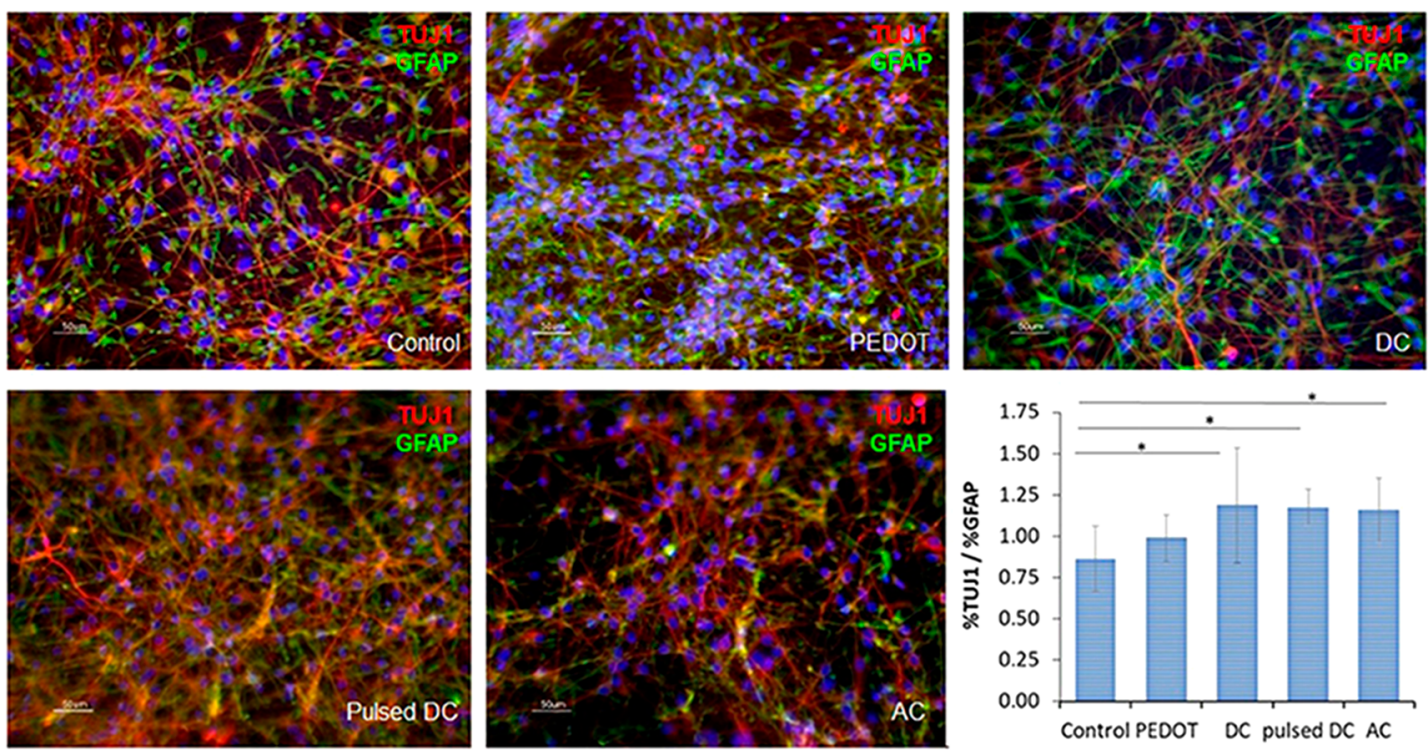

Figure 57. Fluorescence measurements of ReNcells-VM cultured on PEDOT:PSS cross-linked with GOPS under different electrical stimulation conditions. Stained for TUJ1 (red), GFAP (green), and DAPI (blue). Control cultures on standard tissue culture plates ("Control") and on PEDOT:PSS cross-linked with GOPS without electrical stimulation ("PEDOT") were used. The ratio between TUJ1\% and GFAP\% was calculated using 8 different images in each condition ( ${ }^{*} p$-value $<0.05$ and scale bar $50 \mu \mathrm{m}$ ). Reproduced with permission from ref 162 . Copyright 2021 The Authors. Licensed under the terms of the Creative Commons Attribution License, https://creativecommons.org/licenses/by/4.0/. 
a

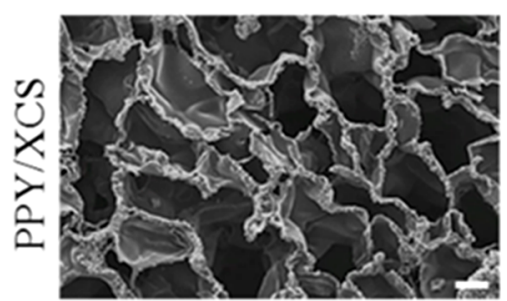

b

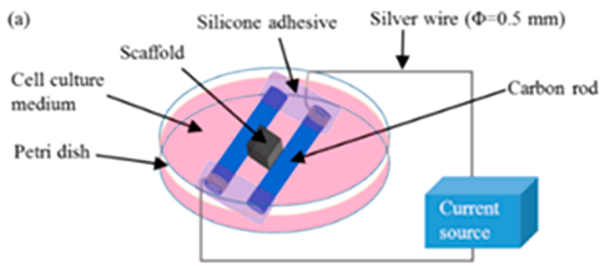

C

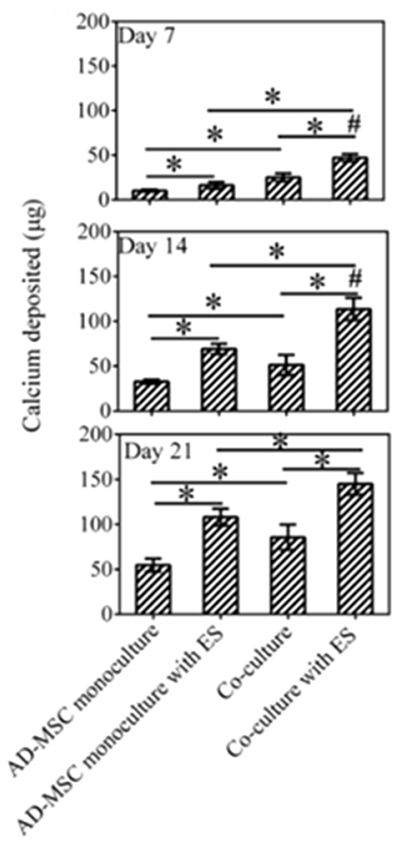

Figure 58. (a) SEM image of the scaffold based on cross-linked chitosan and PPy. (b) Setup for the electrical stimulation. (c) Effect of the electrical stimulation protocol on Calcium deposition at three different times of cell culture. The $*$ and \# marks highlight significant difference $(P<0.05)$ between the designated groups, and a synergistic effect between coculture and ES ( $P$ value of interaction $<0.05$ ), respectively. Reproduced with permission from ref 749. Copyright 2017 John Wiley and Sons.

cells, electrically stimulating stem cells with the use of CPs has been demonstrated to positively impact bone tissue engineering. In this field, several studies have focused on the use of various electrical stimulation approaches and their combined effects with CPs. ${ }^{150,745}$ Many studies adopted precursors cells evaluating the efficacy of the ES protocol at an advanced stage of the differentiation process. Ge and collaborators developed cholic acid doped PPy nanowires and explored their efficacy in promoting osteogenic differentiation of the osteoblastic cell line MC3T3-E1 when combined with electrical stimulation. ${ }^{746}$ Films of electrically conducting polypyrrole combined with heparin (PPy/Hep) were proven to enhance the expression of osteoblast-specific markers when osteoblast-like Saos-2 cells were stimulated with multiple ES rounds $(200 \mathrm{mV} / \mathrm{mm}$ ES was applied for three $6 \mathrm{~h}$ periods over 6 days). ${ }^{747}$

However, in this section, we want to focus on the interplay between ES and CPs in directing stem cell differentiation. Neoh and collaborators reported the combined use of PPY and electrical stimulation to achieve increased mineralization when applied to adipose-derived mesenchymal stem cells (ADMSCs). In their study, the authors adopted a scaffold obtained by coating a PCL-based porous substrate, prepared by salt leaching, in combination with a phase separation technique, with PPy by oxidative polymerization. The authors obtained positive effects in terms of both cell penetration and differentiation when the PPy-coated PCL scaffold was combined with electrical stimulation via direct current stimulation (total current: 200 $\mu \mathrm{A}){ }^{748}$ The same authors investigated the effect of ES when applied to human adipose-derived MSC (AD-MSC)/human umbilical vein endothelial cell (HUVEC) coculture on a conductive polypyrrole (PPy)/chitosan (CS) scaffold (Figure $58) .{ }^{749}$ When ES (200 $\mu \mathrm{A} \mathrm{DC}$ for $4 \mathrm{~h}$ per day) was applied to AD-MSC/HUVEC coculture, the amount of deposited calcium increased by $86 \%$ compared to that of the coculture without ES and by $346 \%$ compared to that of $\mathrm{AD}$-MSC monoculture when no ES was applied.

In a different approach, Wei and colleagues explored the effect of the change in conformation actuated by electrochemically switching the patterned substrate on the differentiation of mesenchymal stem cell (MSC) (Figure 59). ${ }^{750}$ Figure 59a, illustrates the strategy behind this work. Briefly, a PPY array was fabricated, and it was electrochemically switched between highly adhesive hydrophobic nanotubes and poorly adhesive hydrophilic nanotips, and in so doing cell surface adhesion was altered. This change resulted, at the cellular level, in the dynamic attachment and detachment of MSCs and was then translated into mechanotransduction activation and consequently into enhanced differentiation (Figure 59b). The fluorescence intensities of BMP2 and BSP (two markers of osteogenic differentiation) were modulated by the number of nanotube/ nanotip cycles. For a number of cycles up to three, the reported increase in fluorescence intensities of BMP2 and BSP suggested increased MSC osteogenic differentiation. Similarly, Cheng et al. demonstrated that ES enhances osteodifferentiation of human dental pulp-derived stem cells. ${ }^{751}$ The PPy films were deposited on tissue culture polystyrene dishes, and electrical stimulation was applied immediately after cell seeding for $4 \mathrm{~h}$ with an electric field of $0.33 \mathrm{~V} / \mathrm{cm}$.

Cui et al. adopted a newly developed conducting scaffold as a drug delivery device. They established a PLGA/HA/PLA-AP/ phBMP-4 composite scaffold (an electroactive scaffold based on triblock copolymer of poly(L-lactic acid)-block-aniline pentamer-block-poly(L-lactic acid) (PLA-AP) with poly(lactic-coglycolic acid)/hydroxyapatite (PLGA/HA) and the plasmid carrying the gene coding for the human bone morphogenetic protein-4 (hBMP-4)). ${ }^{72}$ The PLGA/HA/PLA-AP/phBMP-4 composite scaffold allowed the controlled "delivery" of the hBMP-4 gene upon electrical stimulation and therefore control of the cell behavior. 
a

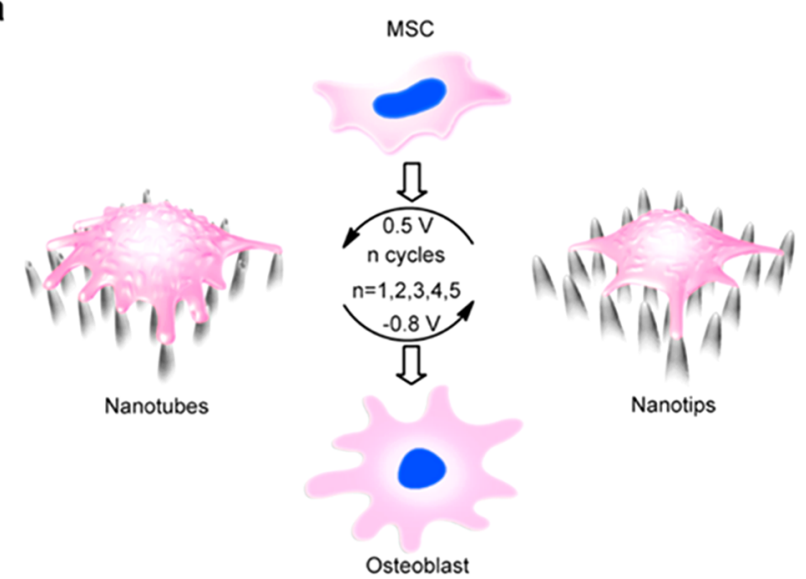

b
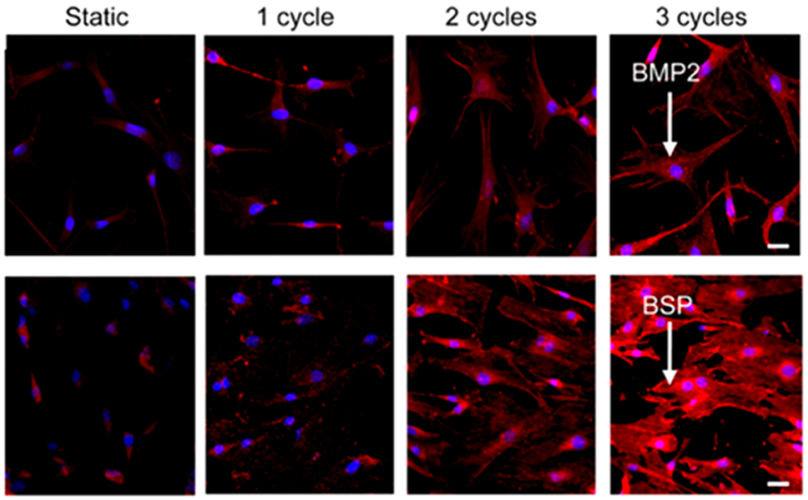

Figure 59. Electrical stimulation of mesenchymal stem cells via electrochemical switching of PPy nanotubes. (a) Schematic of the experiment and functioning of the material. (b) Immunostaining of MSCs cultured on nanotubes for 7 days after experiencing an increasing number of cycles of electrochemical switching from nanotube to nanotip. The top row refers to the osteogenic protein BMP2 and the bottom row to BSP. Scale bars $20 \mu \mathrm{m}$. Adapted with permission from ref 750. Copyright 2017 American Chemical Society.

\section{MONITORING BIOMARKERS IN CELL CULTURES}

Chemical sensing of cellular activity has been the subject of study for decades. ${ }^{753}$ Sensors that can accurately detect biomarkers related to the physiological or pathophysiological state of a cell are in great demand, not only for advancing current in vitro assays but also for early stage diagnostics, drug screening, and even environmental monitoring. ${ }^{754,755}$ Given their accuracy and noninvasive nature, electrochemical detection methods have been the method of choice for cell-based investigation, also enabling real-time analysis of living cells in vitro. ${ }^{756}$ With the advent of microfabrication and microfluidic technologies and the application of nanotechnology, new types of miniaturized electrochemical biosensors have emerged, driven by the need for high throughput and multiparameter detection. ${ }^{757}$ Although there are not many studies on organic bioelectronics for biomarker monitoring in cell cultures, their increased adoption in other complex biological fluids highlights their potential. ${ }^{29}$ From a technological and application point of view, organic electronic materials show almost unmet versatility as they can be easily modified by entrapping enzymes, receptor-like proteins, antibodies, and nucleic acids advancing their sensitivity and selectivity.

This section will cover the state-of-the-art CP-based sensors for measuring cell biomarkers (i) present on the surface of cells or (ii) excreted by the cells under different stimuli conditions or as part of their metabolic activity. Such sensors can be directly embedded in cell cultures or used for downstream monitoring of secreted biomarkers in media with or without cells.

\subsection{Biomarkers Relevant to Cell Activity}

Changes in cellular physiology can affect the expression and secretion profiles of various biomolecules. For example, abnormal cell activity can alter the expression of cell surface biomarkers. Physiological or nonphysiological processes can be reflected not only in changes in cell surface proteins but also in cellular metabolism. These changes can induce local alterations in $\mathrm{pH}$ as well as in the concentration of metabolites or biochemicals, such as dissolved oxygen, glucose, lactate, dopamine, glutamate, or acetylcholine. ${ }^{24,758}$ Hence, detecting cellular metabolites under different stimuli or environmental conditions can give useful insights on the state of cells. For example, acute stress of biological tissues is accompanied by the secretion of inflammatory biomarkers or by alterations in cell metabolism. Monitoring such biochemical changes, especially locally, is somewhat challenging using conventional biological end point assays. Optical methods are still the state of the art in evaluating cellular activity and metabolism; ${ }^{759}$ however they require sample processing and preclude, to some extent,

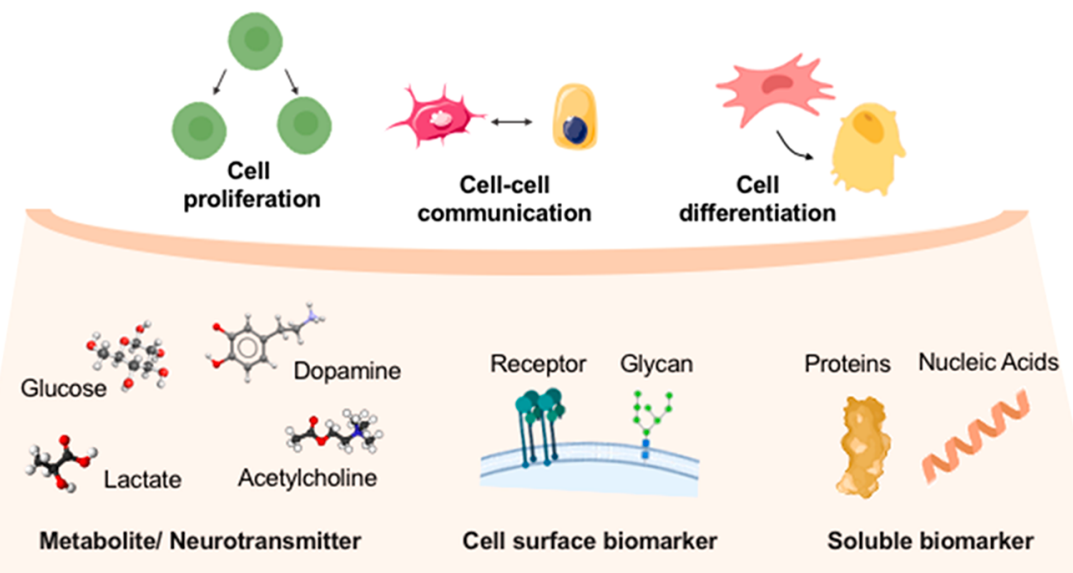

Figure 60. What can we measure from cells with bioelectronic sensors? The scheme gives an overview of the wide range of biomarkers released by cells or present on their surface that can be detected via the sensors developed so far and referenced in this section. Created with BioRender.com. 

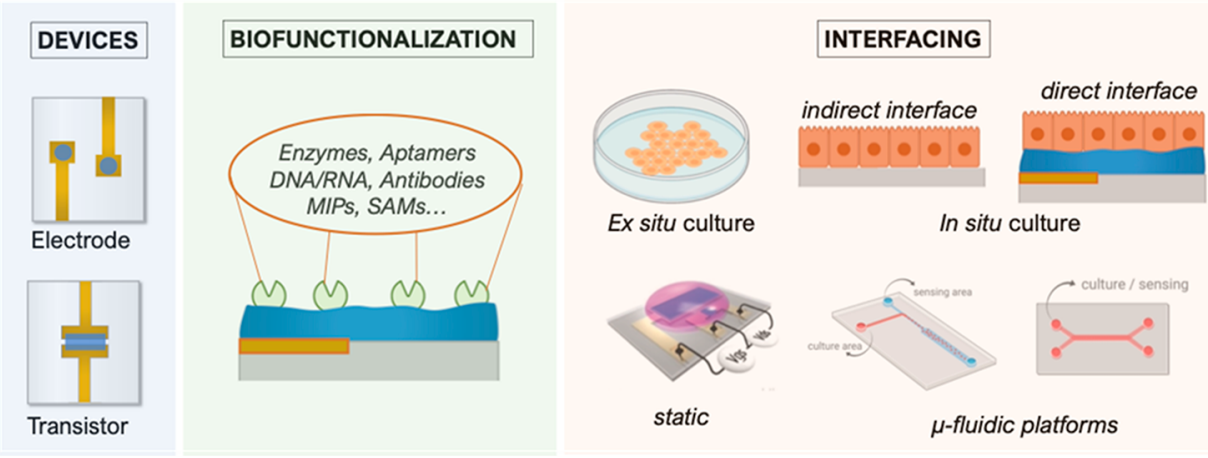

Figure 61. Schematic diagram of the three main aspects of a cell culture organic electronic biosensor, the type of the device, the biofunctionalization method of the sensor, and finally the type of communication (interfacing) between the cell culture and the sensor. Created with BioRender.com.

a

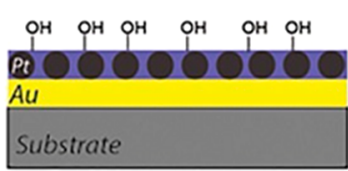

PEDOT:PSS:PVA

b

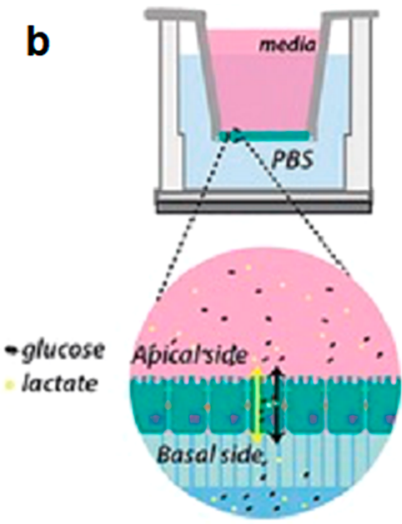

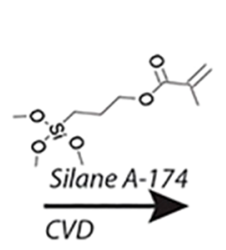
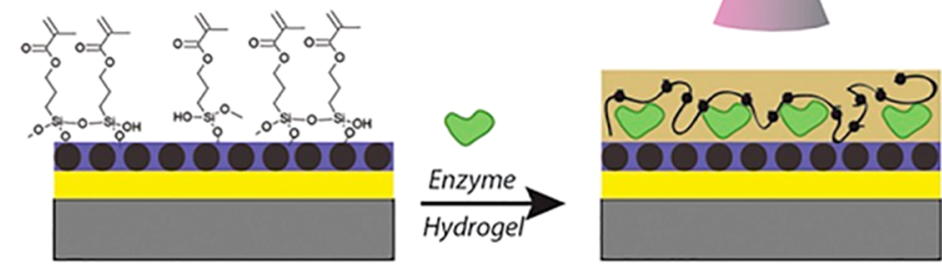

$\approx S$ PEG-DA:PSS
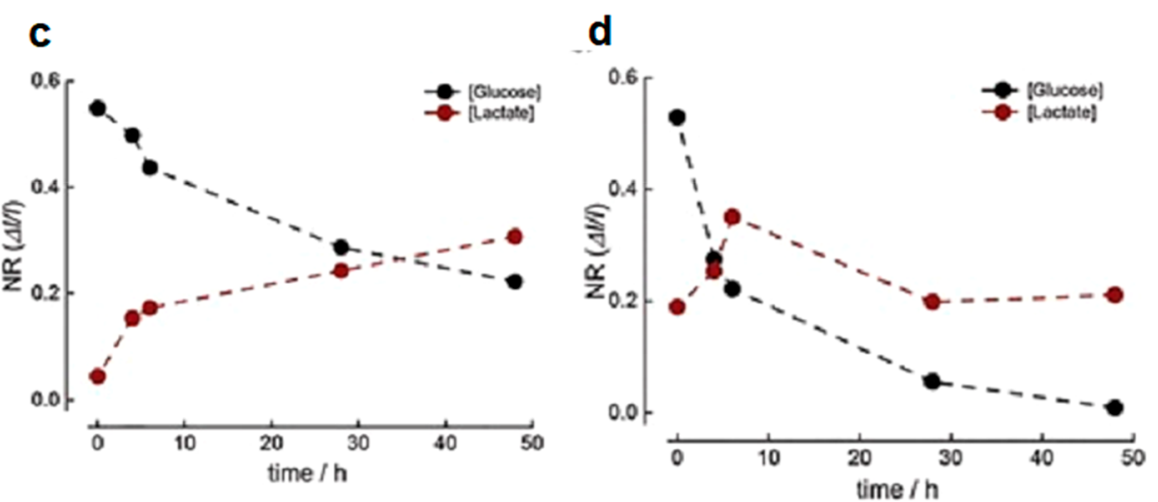

Figure 62. Catalytically enhanced OECTs for monitoring glucose consumption and lactate production from MDCK II cell cultures. (a) Schematic of the biofunctionalization approach based on the entrapment of the enzyme on a UV cross-linked hydrogel, (b) schematic of the MDCK II cell culture setup comprising a typical Transwell insert and glucose (black) and lactate (red) levels over time, as monitored with the OECT based on samples collected from (c) the apical and (d) the basal side of the Transwell insert. Adapted with permission from ref 773 . Copyright 2016 Wiley Periodicals, Inc.

parallelization and real time monitoring. ${ }^{754}$ Electrical sensors on the other hand, having been successfully used to record electrical cellular activity, can be also implemented to sense biomarkers relevant to cell physiology. ${ }^{754,760,761}$ Electrochemical biosensors typically consist of a sensor element that specifically interacts with a biological analyte of interest, producing an electrical signal that is proportional to the analyte concentration. ${ }^{762}$ Benefiting from advances in conventional electronic devices, output signals can be directly processed using electronic circuits, rendering these biosensors very promising for developing integrated cell-culture sensors. Furthermore, the possibility for miniaturization and their ability to provide localized detection of analytes in complex cell cultures makes them highly attractive. Electrode arrays, having been successfully used for electro- physiology recordings, ${ }^{761}$ can be also used to monitor electrochemical reactions (i.e., neurotransmitter release) or enzymatic reactions such as glucose consumption. ${ }^{763}$ Transistors represent another interesting class of electronic devices that can be used for biosensing, as they bear the advantage of transducing the biorecognition events directly into electric signals. Moreover, such a signal can be amplified by the transistor effect as detailed in section 2.3.2. ${ }^{764}$

\subsection{Organic Electronics for Cell-Based Biosensing}

Organic electronic materials have been used as ideal substrates for chemical modification and biorecognition element immobilization (see Figure 60) because of their flexibility and tunability to be able to work with high performance in biological 
milieux. ${ }^{29,64,765,766}$ Changes in the doping state of CPs in particular can be triggered by chemical, electrical, or biological events hence showing these materials' great potential for applications in biosensing. In such approaches, the selectivity and sensitivity of the biosensors can be achieved by establishing an exclusive and intimate interface between the conjugated polymeric transducer and the biorecognition element (e.g., antibodies, enzymes, aptamers, see Figure 61$).^{766,767}$ The transduction mechanism can rely on changes in the polymer redox state induced by the biorecognition event. ${ }^{768}$ Mixed conduction in CPs is a key property that has enabled several new modes of operation and devices. ${ }^{123,124,264}$ CPs can mediate the charge transfer between the electrode and the redox active center of the enzyme, showing promise for the design of amperometric biosensors. In affinity sensors, CPs can be applied as immobilization matrices (e.g., direct or indirect binding of the biorecognition element), signal transduction systems due to their high conductivity (e.g., channel material in OECTs), and even analyte recognizing structures based on, for example, molecular imprinting. Electrochemically deposited CPs with entrapped biorecognition proteins (e.g., receptors, antibodies, antigens) have been applied for the design of various types of affinity sensors. ${ }^{769-771}$

\subsection{State of The Art of Cell-Based Biosensors}

4.3.1. Sensing Secreted Metabolites from Cells. Cellular metabolite sensing is based on enzymes, which specifically catalyze the metabolic reactions. For example, glucose is typically detected using glucose oxidase (GOx), a redox enzyme catalyzing glucose oxidation. This reaction is coupled with the reduction of $\mathrm{H}_{2} \mathrm{O}_{2}$, which is a side product of the enzymatic reaction using $\mathrm{O}_{2}$, the natural cosubstrate of the enzyme. ${ }^{772}$ OECTs with enhanced $\mathrm{H}_{2} \mathrm{O}_{2}$ electrocatalytic activity have been realized by electropolymerizing platinum $(\mathrm{Pt})$ nanoparticles (NPs) on the gate electrode and used to monitor both glucose consumption and lactate production in cell cultures (MDCK-II) over time (Figure 62). ${ }^{773}$ Owing to the high surface area of the NPs and the high specificity of the used oxidase enzymes, the authors achieved sensitive detection of the two metabolites from cells grown in conventional Transwell inserts. This approach, however, suffers various drawbacks, one being the dependence on $\mathrm{O}_{2}$ availability (to generate $\mathrm{H}_{2} \mathrm{O}_{2}$ ) and another the potentially compromised specificity especially when multiple metabolites are to be sensed from a single device (all relying on $\mathrm{H}_{2} \mathrm{O}_{2}$ detection). One way to overcome this is to detect directly the electron(s) produced from the enzyme. Using an electron relay, the communication between the enzyme and the electrode (conducting polymer) can be improved in terms of electron transfer. ${ }^{774}$ This approach has been implemented in OECT sensors, by introducing the common electron transfer mediator ferrocene on the gate of the transistor alongside the desired oxidase. ${ }^{775,776}$ Glucose consumption was measured downstream from a microfluidic cell culture device that was used for multiparametric sensing of cellular activity. The OECTbased detection of the glucose produced by the cells was used as a parameter to assess MDCK-II cells' improved differentiation under mechanical stimulation. ${ }^{776}$

As already mentioned, sensor sensitivity in highly complex cell media is a challenge as there are many electroactive molecules that can contribute to the recorded amperometric signal. To overcome this, we recently developed an OECT circuit based on the well-established Wheatstone bridge configuration, where two sensors, one sensitive to the analyte and another with no specificity (Figure 63), endow an inherent background subtraction. ${ }^{777}$ Using this approach, we were able to detect

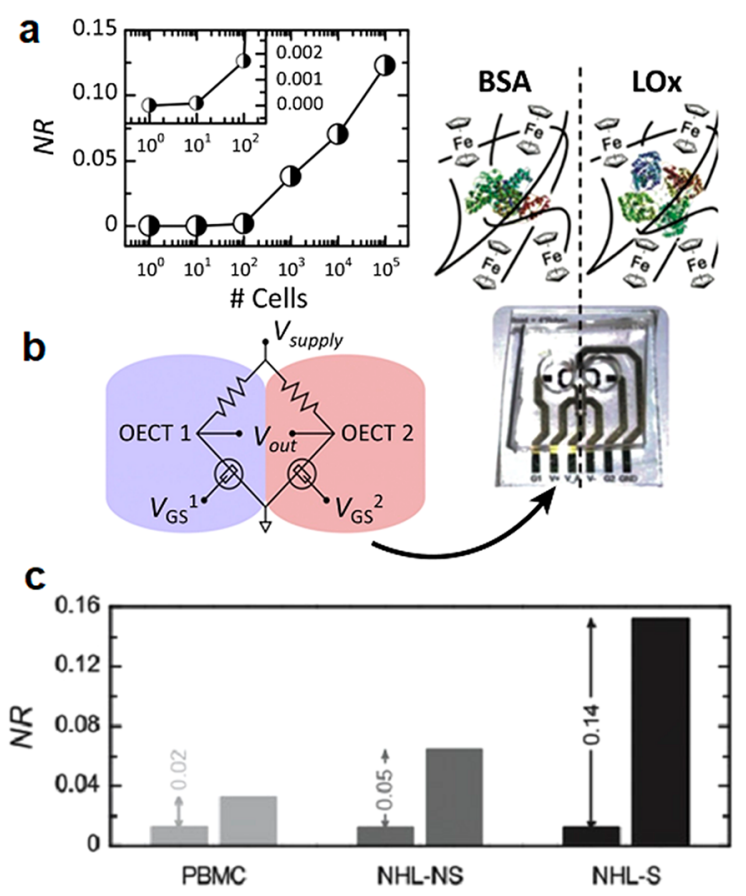

Figure 63. (a) Schematic depiction of the biofunctionalization approach using lactate oxidase. Ferrocene is attached to a polymeric supporting matrix, chitosan. The chitosan ferrocene/lactate oxidase complex is immobilized on the sensing OECT using epoxy, and titration curve of the lactate sensor after successive addition of media is collected from cells cultured at different concentrations. (b) Comparative responses (from the chronoamperometric measurements) of lactate production from peripheral blood mononuclear cells versus unstimulated and stimulated primary non-Hodgkin's lymphoma cells (left to right). Adapted with permission from ref 777 . Copyright 2017 WILEY-VCH Verlag GmbH \& Co. KGaA, Weinheim.

lactate from as low as a few tens of cells derived from samples of human volunteers. These biosensors were also able to discriminate between cancer and healthy cells based on the levels of lactate detected, paving the way for a point-of-care device to be used in cancer diagnostics.

Many neurotransmitters are inherently electro-oxidizable at specific potentials. For example, epinephrine or dopamine can be easily electro-oxidized at relatively low potentials, but numerous other biomolecules (the classic one being ascorbic acid) can be also oxidized at the same low potentials, which prevents this approach from being selective. In order to ensure specificity to dopamine, carboxylated polypyrrole nanotubes (CPNTs) functionalized with an aptamer were deposited on interdigitated electrodes. The resulting liquid-ion gated fieldeffect transistors showed remarkable sensitivity and selectivity to dopamine with a minimum detection level of $100 \mathrm{pM}$, which could be tuned by controlling the diameter of the CPNTs and hence their capacities for aptamer conjugation (Figure 64a). ${ }^{778}$ Micropatterned PEDOT:tosylate microelectrodes were developed for the detection of potassium-induced neurotransmitter release from neuron-like cells. The $\mathrm{CP}$ electrodes exhibited high capacitance per unit area compared to conventional microelectrodes and the capacity to oxidize a wide range of neurotransmitters. Finally, their ability to measure the release of dopamine directly from a group of PC12 cells was 
a

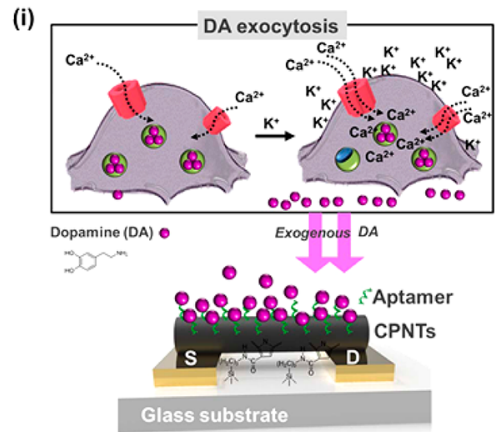

b

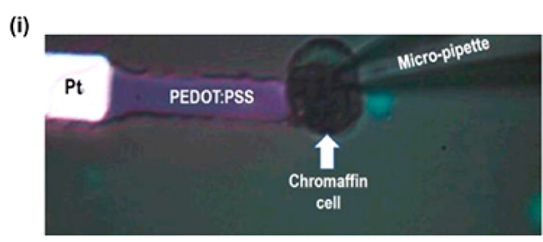

(ii)

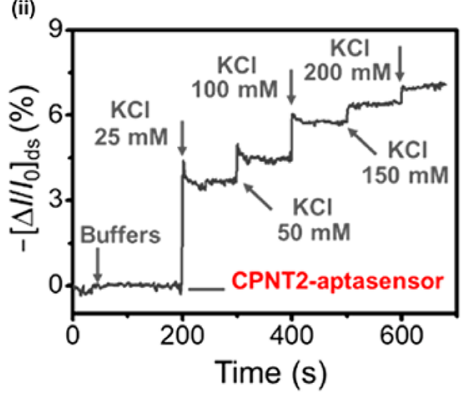

(ii)

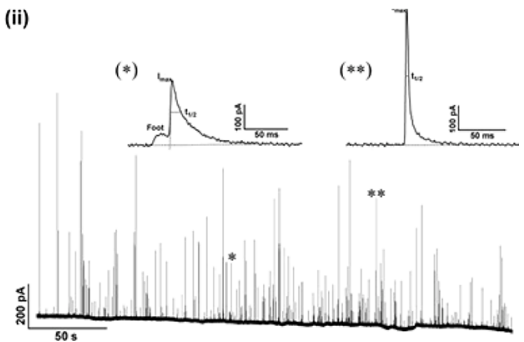

Figure 64. (a) Schematic showing dopamine release mediated by the exocytosis process in PC12 cells via rapid $\mathrm{Ca}^{2+}$ reflux accelerated by $\mathrm{K}^{+}$ions and illustration of the liquid-ion gated aptasensor-functionalized FET using aptamer-conjugated conducting polymer nanotubes (CPNTs) and (ii) chronoamperometric response of the sensor to dopamine for various buffer concentrations. Adapted with permission from ref 778 . Copyright 2020 Springer Nature under Creative Commons Attribution 4.0 license, http://creativecommons.org/licenses/by/4.0/. (b) (i) Photograph of the device setup showing a micropipette that was used to mechanically stimulate the exocytotic process by gently pressing on the chromaffin cell and (ii) electrophysiological recordings of the cell activity showing two distinct amperometric responses that correspond to the neurotransmitter release. Adapted with permission from ref 780. Copyright 2011 WILEY-VCH Verlag GmbH \& Co. KGaA, Weinheim.
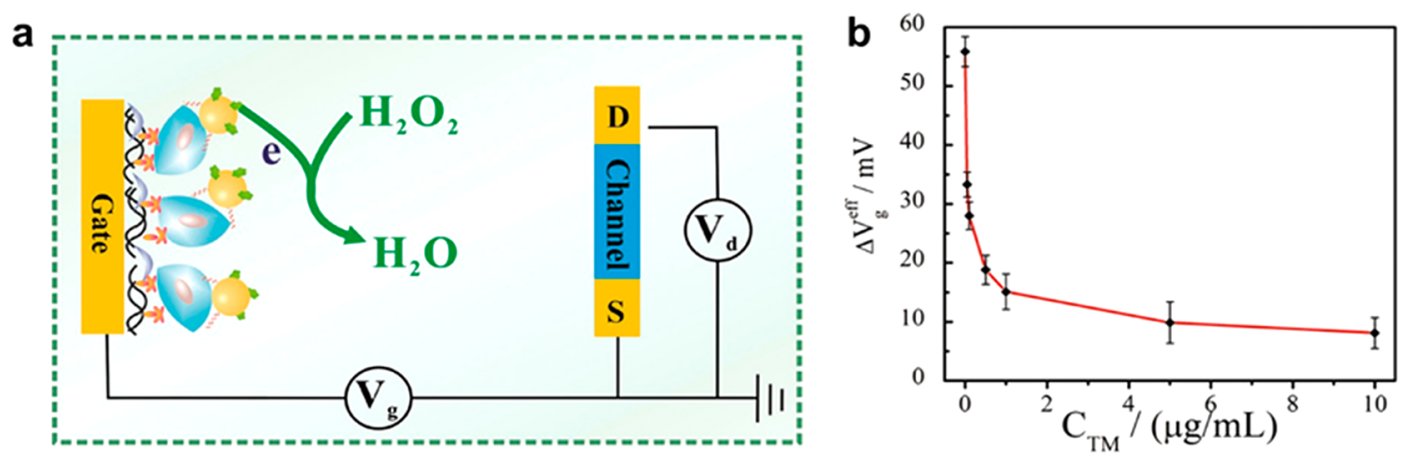

Figure 65. Different approaches to detect glycans as cell surface biomarkers. (a) OECT-based biosensor functionalization for (b) sialic acid sensing. The biorecognition element APBA was modified on MWCNTs to specifically capture sialic acid. Adapted with permission from ref 785 . Copyright 2018 American Chemical Society.

demonstrated in response to a series of stimulations with high $\mathrm{K}^{+}$ buffer. $^{779}$ The (nonspecific) oxidation of catecholamines secreted by chromaffin cells during exocytosis has been successfully demonstrated using PEDOT:PSS MEAs exhibiting high signal-to-noise ratio at a single cell-level (Figure 64b). ${ }^{780}$

Finally, ions can be sensed using ion-selective devices based on inorganic or organic materials. Ion-selective OECTs have been developed, for example, via the incorporation of ionpermeable membranes, reaching significantly higher sensitivity than using conventional potentiometric sensors. ${ }^{781}$

4.3.2. Sensing Cell Surface Biomarkers. As pointed out in section 3.1, the plasma membrane is highly glycosylated. Glycans play crucial roles in a wide variety of biological processes regulating cellular function during development, differentiation, and survival. Abnormal glycan expression levels have been related to several diseases such as cancer. To date, various methods have been used for glycan analysis including mass spectrometry, nuclear magnetic resonance, and high- performance liquid chromatography, ${ }^{782}$ and more recently OECT technology has emerged as a simple method for sensing glycan expression on cell surfaces with high sensitivity. ${ }^{783,784}$

Chen et al. reported the first OECT-based biosensor for the detection of glycan expression directly on living cancer cells (Figure $65 \mathrm{a}(\mathrm{i}))^{785}$ through the detection of the glycan biomarker mannose, the altered expression of which has been linked to cancer growth. ${ }^{786}$ The detection was based on the immobilization on the OECT gate of concanavalin A, a lectin that specifically recognizes mannose on human breast cancer cells (MCF-7). When the cells were captured on the OECT gate, the $\mathrm{H}_{2} \mathrm{O}_{2}$ generated was catalyzed by horseradish peroxidase HRP (conjugated to Au nanoparticles and coimmobilized on the gate electrode). The resulting OECT response was therefore proportional to the concentration of the captured cells. This OECT-based biosensor showed a LOD down to 10 cells $/ \mu \mathrm{L}$ and can also be used to selectively monitor the change of mannose expression on the cell surface upon treatment with an N-glycan 
a

(i)

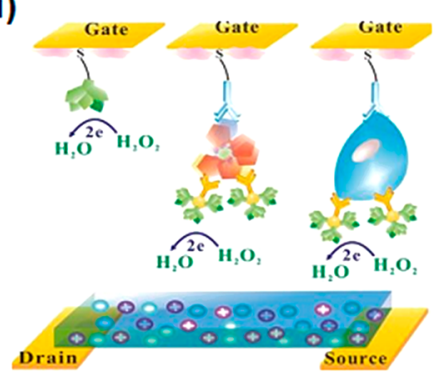

b

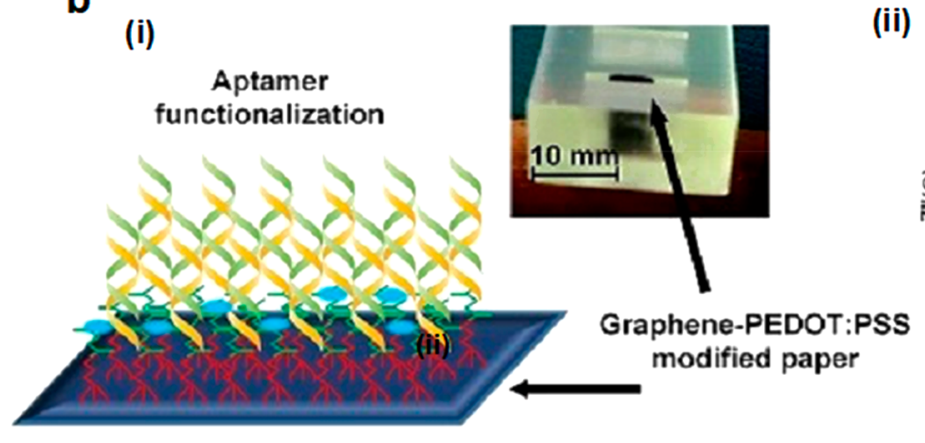

(ii)

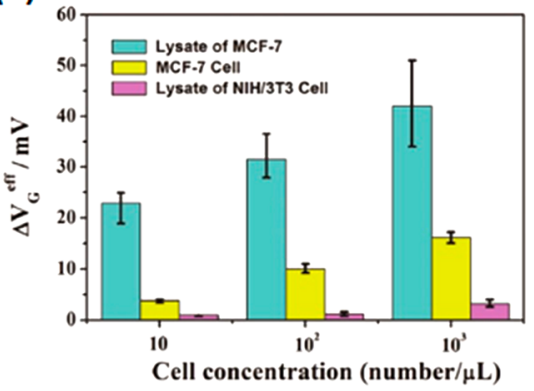

(ii)

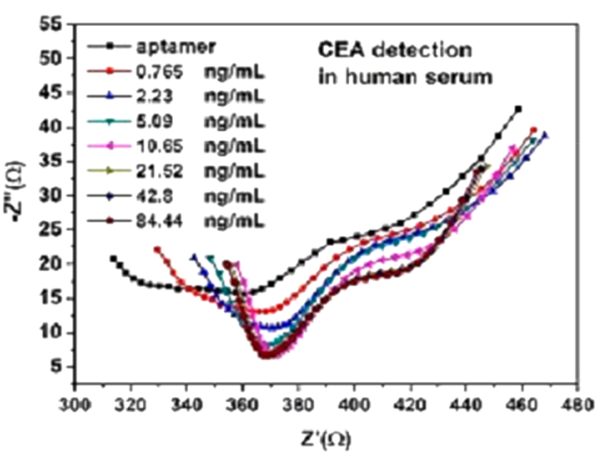

Figure 66. Different strategies to detect protein biomarkers. (a) (i) OECTs with 3 differently functionalized gates. Gates were modified with HRP, HER2 protein, or whole cancer cells. (ii) $\Delta V_{\mathrm{G}}^{\text {eff }}$ of the devices for the detection of HER2 biomarker in different cell lysates or whole cells. Adapted with permission from ref 789. Copyright 2017 WILEY-VCH Verlag GmbH \& Co. KGaA, Weinheim. (b) (i) Graphene/PEDOT:PSS modified paper electrode functionalized with an aptamer against carcinoembryonic antigens (CEA) and (ii) impedance of the electrode at different concentrations of CEA in human serum samples. Adapted with permission from ref 108. Copyright 2020 MDPI, Basel, Switzerland, under the terms and conditions of the Creative Commons Attribution 4.0 license, http://creativecommons.org/licenses/by/4.0/.

inhibitor (Figure 65a(ii)). The aforementioned sensing strategy was further employed to detect mannose and galactose on HeLa cells. ${ }^{787}$ In a follow-up work, a similar detection mechanism was used to detect sialic acid levels in serum samples by adopting 3aminobenzeneboronic acid (APBA) as the biorecognition element. ${ }^{788}$ The gate electrode was modified with carboxylated multiwalled carbon nanotubes to covalently bind APBA, which specifically recognizes sialic acid and therefore affects the gating of the transistor. The transistor showed a sialic acid concentration detection range of 0.1 to $7 \mathrm{mM}$, which fits well with the practical levels of sialic acid in samples from cancer patients and normal control. This biosensor also showed impressive selectivity with low responses toward other glycans: glucose, mannose, lactose, fructose, sucrose, maltose, galactose, and ribose. The work reported so far demonstrated the capabilities of OECT-based biosensors in sensing cell surface biomarkers and how different types of nanomaterials (i.e., $\mathrm{Au}$ nanoparticles, multiwall carbon nanotubes) have been explored to increase the detection sensitivity (Figure 65b). ${ }^{785}$ Overall, bioelectronic sensors are a promising approach for the selective and sensitive detection of glycans as biomarkers, and they hold great promise for clinical application. However, current work is mostly focused on the evaluation of glycan concentration, rather than exploring the promising clinical application of bioelectronics.

Protein biomarkers are the most frequently studied cellular biomarkers, and their detection has been used for differential diagnosis, determination of prognosis, prediction of responses to treatments, and monitoring of disease progression. To detect protein biomarkers at low concentrations in physiological environments (e.g., complex media), OECT-based biosensors appear, due to their inherent amplification property, as promising methods to meet this challenge.

$\mathrm{Fu}$ et al. reported the first OECT-based biosensor that can sensitively detect the cancer protein biomarker human epidermal growth factor receptor 2 (HER2) (Figure 66a). ${ }^{789}$ The devices are operated by detecting electrochemical activity on the gate electrode, which bears catalytic nanoprobes consisting of $\mathrm{Au}$ nanoparticles conjugated with HRP and the HER2 detection antibody. ${ }^{790}$ Upon binding, the electrochemical reaction of the HRP affects the gating efficiency, inducing an OECT response that is proportional to the concentration of HER2. The device was able to detect HER2 expressed on the surface of MCF-7 cells. Overall, the developed protein OECT sensors showed a LOD down to $10^{-14} \mathrm{~g} / \mathrm{mL}$, which is several orders of magnitude lower than the detection limits of other electrochemical approaches (e.g., cyclic voltammetry). As shown in Figure 66a(ii), the OECTs can successfully differentiate breast cancer cells (MCF-7) from normal cells (NIH/3T3) at various cell concentrations. This work paves the way for developing highly sensitive and low-cost biosensors for in-line sensing of protein biomarkers on the surface of living cells.

Using an aptamer as biorecognition element, a graphene/ PEDOT:PSS modified paper-based electrode was used to detect carcinoembryonic antigens (CEAs) using EIS. ${ }^{790}$ The low-cost paper-based aptasensor responds to CEA in the linear range of $0.77-14 \mathrm{ng} / \mathrm{mL}$, with a LOD of $0.45 \mathrm{ng} / \mathrm{mL}$ and $1.06 \mathrm{ng} / \mathrm{mL}$ for CEAs in PBS and human serum, respectively (Figure 66b), showing good correlation with the values reported in a standard 
immunoassay detection method. Apoptosis is the cellular mechanism that contributes to the removal of damaged or dangerous cells. ${ }^{791}$ Caspase- 3 is a protease, and it is an important biomarker of cell apoptosis that participates in the pathogenesis of cancer and neurodegenerative diseases. ${ }^{792}$ OECT-based biosensors have been developed for the detection of caspase- 3 in cancer cells. ${ }^{793}$ Peptides modified on gold nanoparticles on the gate electrode acted as "baits" for caspase-3, which upon binding selectively cleaved them. Upon cleavage, hence peptide removal, the OECT transfer curves shifted to higher voltages. The LOD for such a device goes down to $0.1 \mathrm{pM}$, being able to detect apoptosis in HeLa cells at a concentration of 10 apoptotic cells per $10 \mu \mathrm{L}$ (Figure 66c). Control experiments using caspase9 and BSA showed no distinct shift in the transfer curves, highlighting the selectivity of the developed approach.

\subsection{Characteristics and Challenges of Cell-Based Biosensors}

The use of biosensors as analytical tools for sensing of biomolecules either secreted by cells or present on their surfaces is not straightforward. There are still many challenges to be addressed in order to successfully integrate parallelizable electronic systems with cell-culture environments.

4.4.1. Cell culture: Biosensor Positioning. Biological assays are most often based on cell populations instead of a single cell; hence they correspond to averaged cellular responses. Forcing the cells close to or atop the sensing electrodes is a common strategy to limit the distance the released products have to travel before being detected. Adherent cells can be localized on predefined areas of a culture surface using chemical or physical patterning methods, while nonadherent cells can be localized using techniques such as negative dielectrophoresis or etched microwells. ${ }^{794}$ Addressing the challenge of single-cell resolution for biomarker detection, recent work by Mariani et al. reports the use of carbon nanoelectrodes to realize needle-type OECTs for single-cell analysis. The device was employed as an electrochemical sensor for the proof-of-concept detection of dopamine exhibiting a linear response in the low picomolar range, from 0.002 to $7 \mu \mathrm{M}$ DA with a detection limit of $1 \times 10^{-12}$ M.

Recent work by Strakosas et al. ${ }^{795}$ illustrated the use of a biofunctionalization strategy for continuous detection of utilization of glucose, as measure of cell metabolism directly from cell cultures. The production of $\mathrm{H}_{2} \mathrm{O}_{2}$ (as a byproduct of the GOx enzyme functionalized on PEDOT:PSS based OECTs) results in toxicity to the cells due to the presence of glucose in the cell culture media. A "biostack" functionalization approach was used to layer GOx with PLL and a peroxide scavenging enzyme (e.g., catalase) to prevent toxic levels of $\mathrm{H}_{2} \mathrm{O}_{2}$ from accumulating.

4.4.2. Eliminating the Interference. Eliminating the interference due to nonspecific binding in complex samples, such as cell culture medium, is a key aspect when designing biosensors especially when moving toward multiplexing. ${ }^{29,796,797}$ Zwitterionic polymers, synthetic polypeptides, and PEG-based polymers are reported as low fouling materials, owing to their strong binding to the interfacial hydration layer. ${ }^{798-800}$ Goda and Miyahara developed a series of zwitterionic EDOTs as monomers for the electrodeposition of antifouling $\mathrm{CPs}^{801}$ with poly(EDOT-co-EDOTPC) surface showing 4 times lower nonspecific adsorption compared to pristine PEDOT in $100 \%$ human serum solutions. Hyaluronic acid (HA), bearing repeated disaccharide containing carboxyl groups, hydroxy groups, and amide groups, has been also reported as an antifouling material. Copolymerized PEDOT/ HA composites (coupled with a specific antibody) have been used to selectively detect the cancer biomarker carcinoembryonic antigen with high sensitivity. ${ }^{802}$

Overcoming complex and time-consuming synthetic processes, recently a PEDOT:PSS-based OECT biosensing platform integrated with microfluidics was developed for simultaneous screening of glucose, lactate, and cholesterol in biofluids. The interference issues were resolved by operating the device at a bias far below the oxidation potential of the electroactive species present in the biological milieu. ${ }^{803} \mathrm{~A}$ recent study adopted resonance Raman spectroscopy to probe subtle molecular structural changes of PEDOT:PSS associated with its doping level, alongside electrochemical measurements. The sensitivity of this structural probe in detecting glucose was unaffected when the electrolyte was changed to cell culture media, underlying the specificity of this approach. ${ }^{804}$

4.4.3. Improving Device Performance. Common biosensing approaches are based on direct amperometric chemical reactions happening within the OECT electrolyte with direct charge transfer between the analyte and the $\mathrm{CP}$, which results in subunity transduction of gate to drain current. Hence, amperometric OECTs do not truly display current gains. By separating the chemical transduction and amplification processes on two different electrochemical cells, the Salleo group managed to fully leverage the large transconductance of OECT thus enabling truly high-gain chemical OECT transducers. $^{805}$ They demonstrated the inherent advantage of potentiometric OECT sensors over conventional amperometric OECTs by utilizing enzymatic reactions of GOx to detect glucose, achieving a good dynamic range and low OECT OFF currents of $30 \mathrm{nA}$.

\section{ON-CHIP OR FLUIDIC-INTEGRATED IN VITRO MODELS}

An abiding challenge of in vitro models is to successfully replicate the precise microenvironment found in the human body. For example, cells grown in static conditions lack the right physical cues and gradients of nutrients and oxygen. The incorporation of microfluidic technologies allows investigation of cellular microenvironments ensuring a laminar fluid flow that mimics the in vivo situation with high accuracy, low reagent consumption, and low waste generation. ${ }^{11,806}$ Dynamic fluid flow has also been reported to create subtle shear stress that directs cell proliferation and differentiation, formation of functional structures, and release of chemical factors. Microfluidics are typically made of polydimethylsiloxane (PDMS), ${ }^{807}$ poly(methyl methacrylate) (PMMA), ${ }^{808,809}$ polycarbonate (PC), ${ }^{810}$ and cyclic olefin polymer (COP). ${ }^{811-813}$ Benefits and limitations of their use have been reviewed elsewhere. ${ }^{814,815}$

Microfluidic-based cell cultures and OoCs have made strides in the past decade as in vitro models, with the prospect of reducing or replacing animal models being closer than ever. $^{816,817}$ Although the majority of organ models combine perfectly microfluidic technologies and tissue engineering, ${ }^{5}$ new approaches have recently emerged recognizing the additional value of incorporating biosensing elements ${ }^{818,819}$ that will allow in situ monitoring of cellular processes ${ }^{16,810}$ and metabolites 820 and drug delivery ${ }^{821}$ and detection. ${ }^{822}$ The expansion of the OoC field has raised the necessity for the coupling of these organ models with reliable in-line electrochemical sensors capable of providing label-free, real-time multiparametric information on 
a

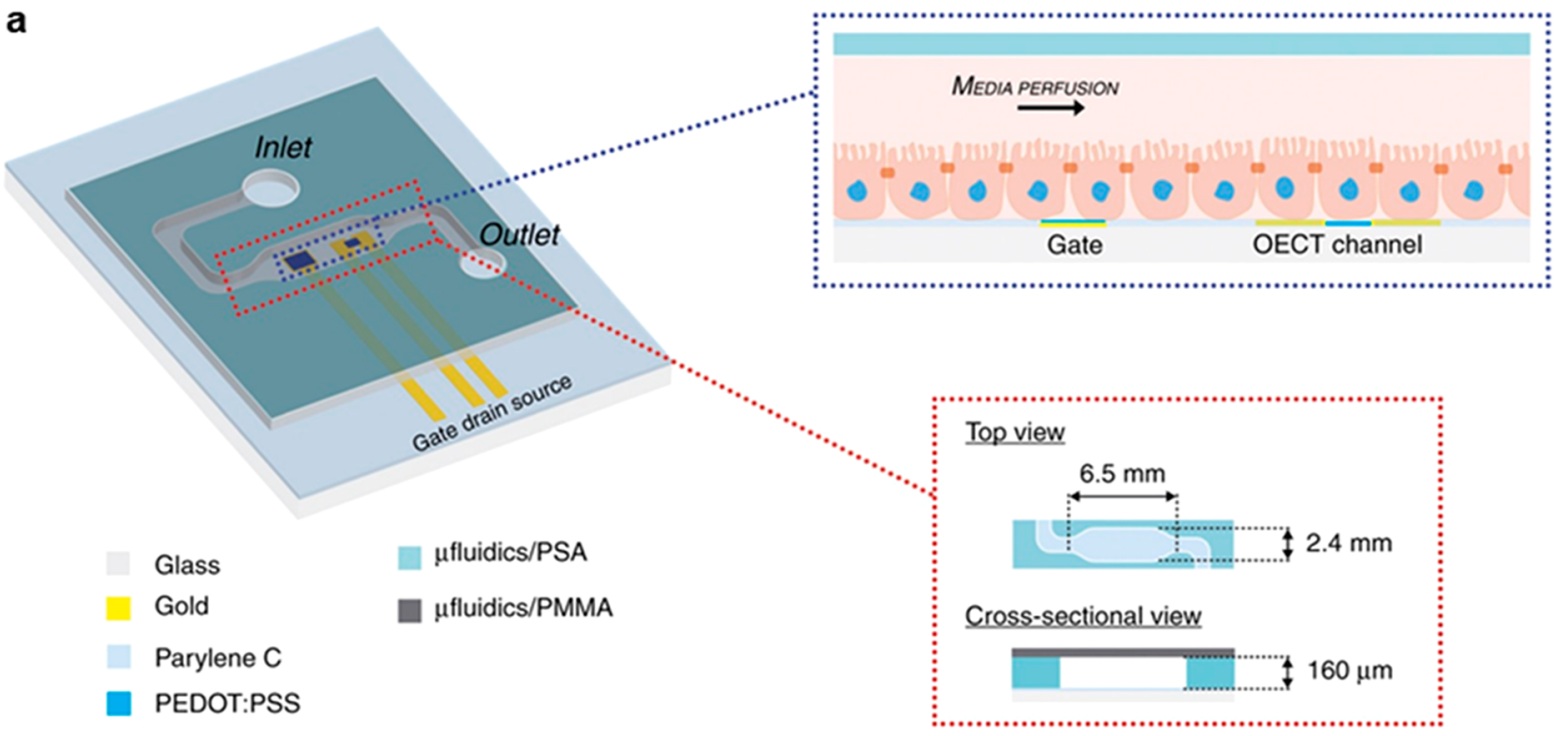

b

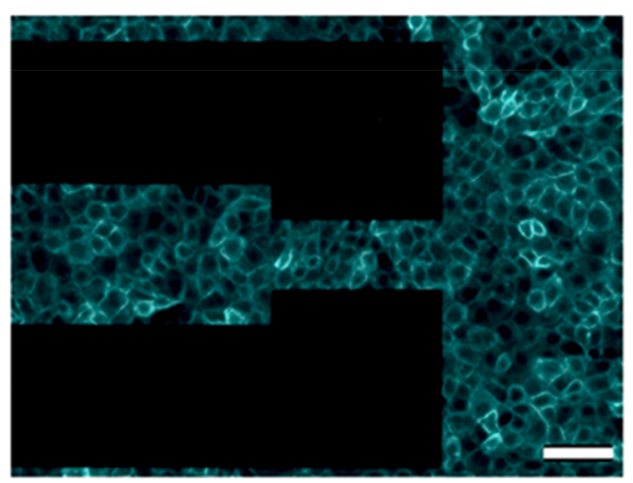

C

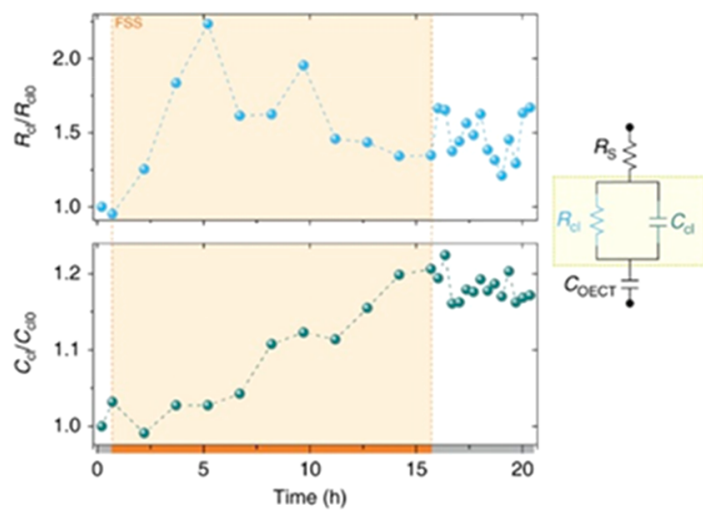

Figure 67. (a) Microfluidic chip with integrated OECT. Illustration showing the OECT device along with the cell monolayer lining the surface. Top and cross-sectional views showing the architecture of the bioelectronic microfluidic device. (b) Immunofluorescence image of MDCK II cell layer formed inside the channel of the OECT in the microfluidic chip. (c) Graphs showing the data $\left(R_{\mathrm{c}}, C_{\mathrm{cl}}\right)$ generated via in-line monitoring over $15 \mathrm{~h}$ under flow conditions. $R_{\mathrm{cl}}$ and $C_{\mathrm{cl}}$ calculated using the depicted equivalent circuit. Adapted with permission from ref 281 . Copyright 2017 Nature Licensed under Creative Commons Attribution 4.0 International (CC BY 4.0) License, https://creativecommons.org/licenses/by/4.0/.

cellular processes. ${ }^{40,53}$ Models of bone- ${ }^{823}$ heart-,${ }^{824}$ vasculature-, ${ }^{602}$ brain-, ${ }^{825-827}$ and kidney-on-a-chip ${ }^{660}$ have been developed to mimic specific tissues while electrically monitoring and sensing cellular processes. This synergy between $\mathrm{OoC}$ and bioelectronics could have a huge impact on advancing in vitro assays.

Different bioelectronic configurations have been adapted to couple microfluidic devices, mostly electrodes and OECTs. The OECT configuration has been extensively used by our group for spheroid impedance sensing, analyte detection, ${ }^{776,828}$ and cell monitoring ${ }^{281}$ inside microfluidic devices, and we were the first to demonstrate coupling between OECTs and microfluidics to achieve multiparametric monitoring of kidney tissue formation. This platform consisted of an OECT made on a glass substrate coupled to a PMMA microfluidic device. The gate electrode and the PEDOT:PSS channel were positioned in the chamber seen in Figure 67. MDCK-II cells were exposed to flow shear stress using a syringe pump. The illustration shows a cross-sectional view of the device, in which the different components are represented: from bottom to top, the glass $/ \mathrm{PaC}$ substrate with the OECT channel and gate, the epithelium, and the media perfusing through the microfluidic chamber. MDCK-II cells expressing red fluorescent protein (RFP)-tagged actin were seeded into the OECT channel, and confocal images were taken when fully confluent cells were grown inside the chamber (Figure 67b). Electrical measurements were taken at different time points to evaluate the evolution of the cell layer resistance $\left(R_{\mathrm{cl}}\right)$ and cell layer capacitance $\left(C_{\mathrm{cl}}\right)$ while the cells were exposed to fluid shear stress. An increment in the $R_{\mathrm{cl}}$ and $C_{\mathrm{cl}}$ values in the first $5 \mathrm{~h}$ at 0.3 dyn $\mathrm{cm}^{-2}$ was attributed to the reassembly of intercellular tight and adherent junctions due to the flow shear stress-induced mechanotransduction (Figure 67c). ${ }^{281}$ This platform clearly highlights the potential to integrate organic bioelectronic components as embedded sensors in microfluidics, without compromising the optical monitoring capabilities. Nevertheless, limitations may arise due to the single fluidic channel architecture and the lateral gate electrode positioning, which may affect efficiency during cell culture. Future developments could benefit from including multilayer microfluidics for mimicking basal and apical compartments for applications in complex cell-based assays such as organ-onchip models.

The adaptability of microfluidics enables different design-topurpose configurations such as trapping elements, ${ }^{660}$ membranes, ${ }^{820,829}$ topographies, ${ }^{830}$ or even an ion pump for drug delivery in vivo. ${ }^{821} \mathrm{~A}$ trapping PDMS-microfluidic device 


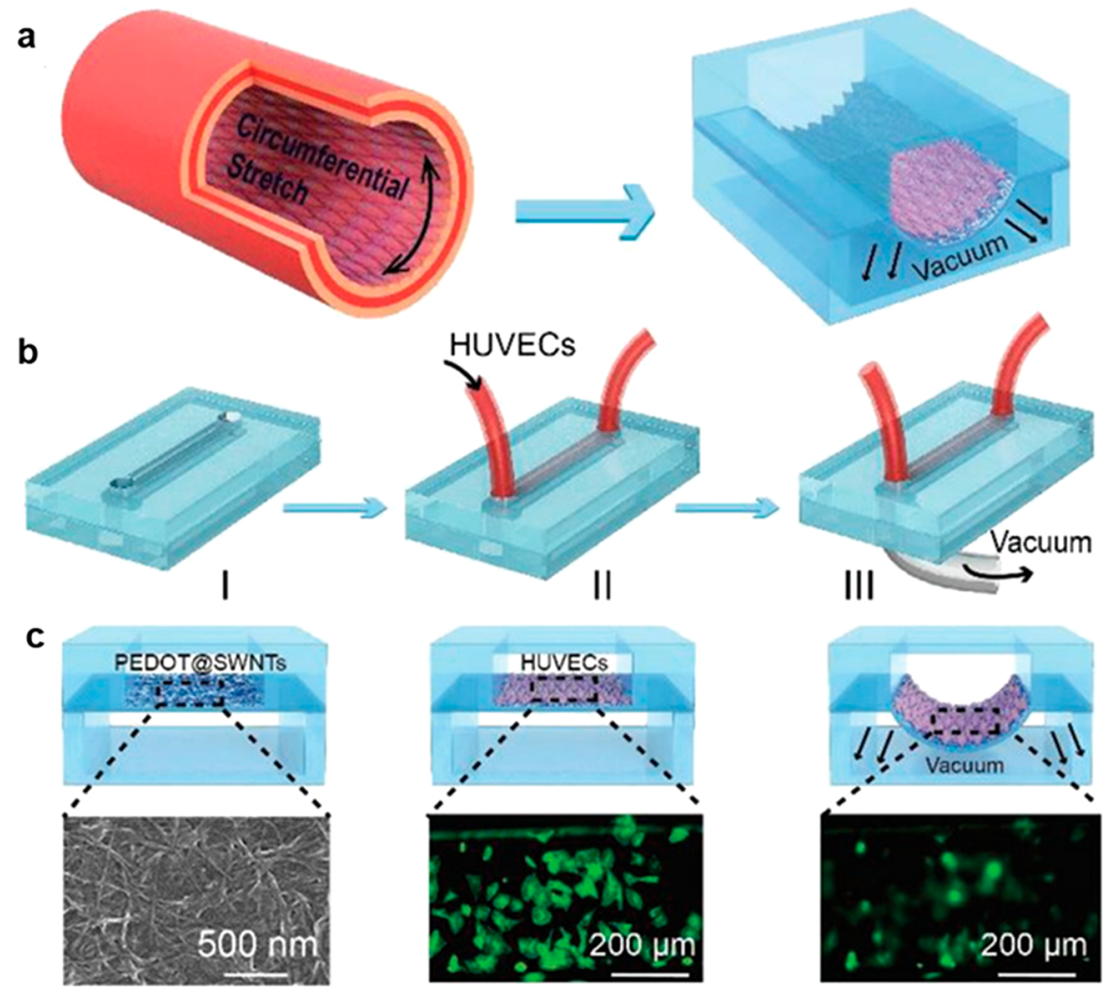

Figure 68. (a) Flexible electrochemical sensor-integrated vascular chip. (b) Schematic diagrams of the cell (HUVEC) seeding and operation process (vacuum induced circumferential stretch) of the fabricated device. (c) Sectional views of the area of interest: (I) SEM of the electrode (PEDOT coated SWCNT) and fluorescence microscopy images of HUVECs (II) without and (III) with circumferential stretch. Adapted with permission from ref 831. Copyright 2010 Wiley-VCH Verlag GmbH \& Co. KGaA, Weinheim.

coupled to a PEDOT:PSS-based transistor was fabricated for the electrical resistance measurement of different spheroid compositions and sizes. The microfluidic device and OECT were fabricated by lithography to ensure proper resolution. The microfluidic device comprised a single straight channel that terminated with a nozzle where the spheroid was positioned. Following the nozzle, a round chamber held the gate electrode. The channel of the OECT was positioned in the straight microfluidic channel. Measuring spheroids from different cell lines (TIFs, MDCK-II, and a combination of TIF plus MDCKII) showed differences in the spheroid resistance values. ${ }^{660}$ Devices capable of sensing metabolite conditions are very important in POC (point-of-care) technologies as they can provide useful insights for the prevention of diseases. In this sense, a finger-powered microfluidic device coupled to multiplexed OECTs was developed for the POC sensing of three critical biomarkers: lactate, glucose, and cholesterol in saliva. The device comprised a three OECT configuration plus a control OECT integrated into a PDMS-based microfluidic device. The fluid flow microfluidic device was activated by pressing an activation button with the finger on the top of the microfluidic device. Limitations of the system were the electrical crosstalk between OECTs and the differences in the currentresponse times for different OECTs due to the spatial resolution. Despite these limitations, the biosensor possessed excellent analytical performance with detection ranges that covered physiological ranges in saliva. ${ }^{776}$ Regarding bioelement sensing, nanofibers can be integrated as sensor elements, as done by Chen and co-workers. ${ }^{828}$ They integrated a PEO/PEDOT:PSS nanofiber into an ITO electrode inside a PDMS-based microfluidic device for the capture and release of circulating tumor cells (CTCs). The platform could capture targeted
EpCAM-positive cells (transmembrane glycoprotein with oncogenic potential) but also efficiently purify CTCs from an EpCAM-negative cell solution through the application of a cyclic potential electrical stimulation.

Moreover, stretchable microfluidics made use of the inherent ability of PDMS to contract under shear stress to generate a mechanical motion that will translate to mechanical shear stress for the cells. In nature, endothelial cells (vascular cells) are continuously exposed to dynamic blood flow that creates shear stress and vasodilation. These mechanotransduction phenomena induce morphological and behavioral changes to the endothelial tissue. In this context, Huang and co-workers integrated a stretchable electrochemical sensor inside a microfluidic device to simulate vascular parameters and monitor the biochemical signal produce in the process. The micropatterned sensor is constructed with PEDOT/SWCNTs that satisfy mechanical compression and electrochemical performance against deformation at real-time. To recapitulate the stretched endothelial, a PDMS membrane was built between two microfluidic PDMS layers and the stretch-induced signaling molecules released from the deformed endothelial cells were monitored (Figure 68a). The PEDOT/SWCNTs electrodes were patterned onto the PDMS and placed inside the microfluidic device for real-time electrical monitoring. Human umbilical vein endothelial cells (HUVECs) were seeded on the electrode to form the vascular endothelium. Vacuum was applied to induce elastic deformation of the PEDOT/SWCNTs membrane that stresses adherent cells (Figure 68b). Figure 68c illustrates the concept of the paper showing the SEM image of the integrated membrane (I), and the confocal images of the HUVEC cells cultured in the membrane before (II) and after circumferential stretching (III). ${ }^{831}$ 


\section{PERSPECTIVES}

The applications of bioelectronics including point-of-care diagnostics and electroceuticals have skyrocketed over the past decades and continue to evolve at a high pace. Materials technology has played an organic role during the evolution of bioelectronics, with organic electronics offering a breadth of new possibilities at various levels. Despite their vast potential, organic bioelectronics have still not reached the level of commercial or clinical translation, with the exception of few scarce examples (e.g., Multi Channel Systems, GmbH; MEAs incorporating PEDOT:PSS).

In vivo and on body interfacing applications are far more established compared to in vitro, given the great need for implantable biomedical devices and prosthetics on the one hand and the increased consumer adoption and combination with IoT in the case of wearable sensors on the other hand. Despite being especially promising for the dual purpose of monitoring and stimulating biological systems outside of a living organism in a controlled fashion, in vitro bioelectronics is still less explored and considerably less adopted. This is in part due to the commonly observed "reluctance to change" in the pharmaceutical industry posing unrealistic requirements in order to invest in and adopt early stage technologies leading to a vicious cycle that significantly delays their standardization and real-world implementation. There is therefore a clear need to bridge the gap between $\mathrm{R} \& \mathrm{D}$ and translation.

\subsection{Technological Challenges To Be Overcome}

To realize and standardize biointegrated chips or platforms that can probe biological systems from the molecule to individual cells to whole organism levels, there are technological bottlenecks that need to be addressed. Automation, compatibility with large-scale fabrication, and high throughput capabilities are all necessary to drive this technology forward. Other important challenges lie in the integration and the biological fate of the organic interfaces. In terms of integration, the incorporation of circuitry with sensors, actuators, and acquisition systems, including the development of versatile readout instrumentation and electrical connections, while minimizing manufacturing complexity, is vital. In terms of interfaces, a thorough understanding of the biotic-abiotic coupling is crucial and will result in more stable and long-lasting platforms. In addition, the ability to predict the coupling dynamics would allow iterative improvement of both the electronics and the biological systems. Last but not least, another abiding challenge that impedes standardization of this technology is the interpretation of the electrical readout to meaningful biological information, embracing machine and deep learning, where possible, as these mass producible bioelectronic assays become a significant component of measurement science capability.

\subsection{Opportunities}

Organic electronics, in the same way as carbon-based materials, have intrinsic similarities to the structure of biomacromolecules providing an ECM like environment. This makes them more biocompatible and mechanically compliant with tissues compared to their inorganic counterparts, overcoming limitations such as mechanical stiffness, reduced conformation, and adaptability. In line with the scaling down of in vitro models, the ongoing miniaturization of organic semiconductor devices opens new opportunities in interfacing and probing cells in vitro, delivering biomolecules inside cells, or electrically stimulating cells. Nanoscale organic bioelectronic devices can thus play a fundamental role in studying protein function and its reaction pathways inside the cells or on cell membranes. This opens new opportunities for drug testing and for assessing therapy efficacy at the molecular level as well as for personalized medicine. This is illustrated in section 3 for example, with the prospect of using human cells for cell membrane level studies.

At the tissue interfacing level, significant progress has been made in the area of $2 \mathrm{D}$ electrogenic cell cultures, particularly with respect to the recordings of cell-derived signals. Further efforts in refining these devices and maximizing SNR will aid in the improvement and development of more complex systems in the future. Particular focus should be paid to operating these recording systems in more complex cellular environments. A majority of studies record cellular signals from only a single cell type. However, to fully recreate bodily systems and functions, these measurements need to be able to single out individual signals from a host of cellular phenotypes. Regardless, further development of these devices can allow for a greater understanding of electrogenic cells, pushing toward an ability to truly recapitulate some of these interesting and complex biological systems. The same challenges lie in the case of nonelectrogenic cells, with the additional challenge of the standardization of the measurements and the establishment of the right figures of merits, which is not straightforward as in the case of electrophysiological recordings.

A considerable effort has been also made in promoting neuronal growth using electrical stimulation, but further experiments need to be performed to understand more the underlying biological mechanisms of the cell's behavior. Great strides have also been made in creating in vitro systems for eliciting changes in spiking behavior from neuronal and cardiac cells. Next steps should involve the transduction of an applied stimulus through networks of multiple cells.

The unique possibility of CPs to adopt 3D porous ECM mimicking architectures (scaffolds) has proven to be fruitful in successfully interfacing and supporting complex (3D) cell cultures while providing in line monitoring capabilities. Adaptation of those features in standardized assays (i.e., transwell setups) will offer a direct (and faster) route for commercialization benefiting from existing instrumentation, equipment, and protocols.

Organs-on-chips represent a new technology set to revolutionize the field of cell-based in vitro assays. Despite the great advances in this field, there are still no examples in the literature that recapitulate the complexity of organ-like models able to be monitored in a noninvasive manner and in real-time. We believe that now is time to push forward accurate, reliable, and authentic in vitro models that truly represent the in vivo situation while incorporating in-line electrical sensing of tissue. The market demands miniaturization and parallelization, and the need for multiplexing is the main drive to implement electrical sensors as the most straightforward approach for highlevel sensor integration in such in vitro systems. However, there still remains challenges notably when it comes to directly interfacing cell cultures with biosensors in terms of biofouling, sensitivity, and specificity as well as sensor stability and longterm operation. Three-dimensional CP based scaffolds have the potential to be integrated with microfluidics to meet the scalability, accuracy, and reliability requirements. We expect that the following years will witness such advancements aiding the development of multifunctional biomimetic OoC platforms that could eventually replace animal models in drug discovery and disease research. 


\section{AUTHOR INFORMATION}

\section{Corresponding Author}

Róisín M. Owens - Department of Chemical Engineering and Biotechnology, University of Cambridge, Cambridge CB3 OAS, U.K.; ๑ orcid.org/0000-0001-7856-2108; Email: rmo37@ cam.ac.uk

\section{Authors}

Charalampos Pitsalidis - Department of Physics, Khalifa University of Science and Technology, Abu Dhabi 127788, UAE; Department of Chemical Engineering and Biotechnology, University of Cambridge, Cambridge CB3 OAS, U.K.

Anna-Maria Pappa - Department of Biomedical Engineering, Khalifa University of Science and Technology, Abu Dhabi 127788, UAE; ๑ orcid.org/0000-0002-7980-4073

Alexander J. Boys - Department of Chemical Engineering and Biotechnology, University of Cambridge, Cambridge CB3 OAS, U.K.; ๑ orcid.org/0000-0002-6488-7005

Ying Fu - Department of Chemical Engineering and Biotechnology, University of Cambridge, Cambridge CB3 OAS, U.K.; Department of Pure and Applied Chemistry, Technology and Innovation Centre, University of Strathclyde, Glasgow G1 $1 R D, U . K$.

Chrysanthi-Maria Moysidou - Department of Chemical Engineering and Biotechnology, University of Cambridge, Cambridge CB3 OAS, U.K.; ๑ orcid.org/0000-0001-98092764

Douglas van Niekerk - Department of Chemical Engineering and Biotechnology, University of Cambridge, Cambridge CB3 OAS, U.K.

Janire Saez - Department of Chemical Engineering and Biotechnology, University of Cambridge, Cambridge CB3 OAS, U.K.; Microfluidics Cluster UPV/EHU, BIOMICs Microfluidics Group, Lascaray Research Center, University of the Basque Country UPV/EHU, 01006 Vitoria-Gasteiz, Spain; Ikerbasque, Basque Foundation for Science, E-48011 Bilbao, Spain; ๑ orcid.org/0000-0002-9246-0818

Achilleas Savva - Department of Chemical Engineering and Biotechnology, University of Cambridge, Cambridge CB3 OAS, U.K.; ๑ orcid.org/0000-0002-0197-0290

Donata Iandolo - INSERM, U1059 Sainbiose, Université Jean Monnet, Mines Saint-Étienne, Université de Lyon, 42023

Saint-Étienne, France; ๑ orcid.org/0000-0002-8090-8427

Complete contact information is available at:

https://pubs.acs.org/10.1021/acs.chemrev.1c00539

\section{Notes}

The authors declare no competing financial interest.

\section{Biographies}

Charalampos Pitsalidis is Assistant Professor in the Department of Physics at Khalifa University and visiting researcher at Cambridge University. During his postdoctoral research in Bioelectronic Systems and Technology group at Cambridge, he worked on bridging novel organic electronic devices with biological systems toward next generation in vitro cell culture platforms. His research now at $\mathrm{KU}$ centers on the use of organic and hybrid electronic materials for the development of 3D biomimetic structures and devices for monitoring and controlling biological systems at different length scales and complexity levels. Expanding on this, he focuses on scaffold-based electronics for use in wearable applications.
Anna-Maria Pappa is Assistant Professor in the Department of Biomedical Engineering at Khalifa University. She was previously the Oppenheimer research fellow in the Department of Chemical Engineering and Biotechnology at Cambridge University while simultaneously holding the Maudslay-Butler Research Fellowship in Engineering at Pembroke College, Cambridge. In 2017, she received the L'Oreal-UNESCO Women in Science award, while in 2019 she was listed on the Innovators under 35 MIT technology review. Her current research interests lie in biointegrated electronics and point-of-care sensors.

Alexander J. Boys received his Ph.D. in Materials Science and Engineering at Cornell University. He worked in materials characterization, biomechanics, and tissue engineered implant design through fellowships from the US National Institutes of Health and the National Science Foundation. Alex began work at the University of Cambridge in 2019, developing bioelectronic tissue engineered in vitro systems. $\mathrm{He}$ currently holds a fellowship from the Human Frontier Science Program for the development of bioelectronic implants and implantable materials. His interests involve tissue engineering, bioelectronics, and biomechanics.

Ying Fu is a Lecturer and Chancellor's Fellow in the Department for Pure and Applied Chemistry at University of Strathclyde. She was a research associate in the Department of Chemical Engineering and Biotechnology in the BioElectronic Systems and Technologies group. Dr. Fu holds a Ph.D. degree in Analytical Chemistry, and her research interests include novel biosensor development and application. She is currently working on highly sensitive organic electronics integrated with microfluidics for real time monitoring, aimed at early diagnosis and effective therapy for various diseases.

Chrysanthi-Maria Moysidou is a postdoctoral research associate in the Department of Chemical Engineering and Biotechnology at the University of Cambridge. She received her Diploma (B.Sc. and M.Sc.) in Chemical Engineering, followed by a Masters in Medical Research Methodology, at the Aristotle University of Thessaloniki. Her Ph.D. research was carried out in the Department of Chemical Engineering and Biotechnology of the University of Cambridge where she worked on merging tissue engineering and organs-on-chips with bioelectronics for building in vitro models of human tissues, with a particular focus on the gut-microbiome axis, under the supervision of Prof. Róisin M. Owens.

Douglas van Niekerk is a Ph.D. candidate under the supervision of Prof. Róisin M. Owens and is researching the integration of bioelectronics with tissue engineered research platforms for the study of disease, with a focus on esophageal cancer. Douglas joined the Sensor Technologies and Applications CDT at the University of Cambridge where he completed a Master of Research degree. Prior to that, he received Bachelors degrees in Biomedical Engineering (BEngSc) and Electrical Engineering $(\mathrm{BSc})$ from the University of the Witwatersrand.

Janire Saez is an Ikerbasque Research Fellow and Visiting Professor at the University of the Basque Country (UPV/EHU). Until 2021, she was a Marie Curie Fellow and Research Associate in the Bioelectronic Systems Technology group at the University of Cambridge working on bioelectronics and microfluidics. She has a degree in Chemistry and a Master's degree in Pharmacology from the University of the Basque Country (UPV/EHU). In 2017, she obtained her Ph.D. in Environment and Marine Resources from the same university. During this time, she studied the integration of smart materials into microfluidic devices for environmental water monitoring, fluidic handling, and sensing.

Achilleas Savva is a Marie Curie Fellow in the BioElectronic Systems Technologies group in the Department of Chemical Engineering and Biotechnology at the University of Cambridge. He is a Chemical 
Engineer with a Ph.D. in Materials Science and Engineering. His research interests are focused on the understanding of mixed ionic/ electronic conduction in conjugated polymers as well as on the development of bioelectronic devices for electrical and optical interaction with living systems. He is currently aiming to develop electroactive 3D bioelectronic platforms to monitor and control stem cell proliferation and differentiation with electrical and optical cues.

Donata Iandolo received her Ph.D. in Industrial Biotechnologies in 2010. Her research focus is now on bioelectronics and the use of conducting polymers at the interface with biological systems to both monitor and stimulate them. She was a Marie Sklodowska-Curie fellow until 2018, and currently she is a research fellow at Mines Saint-Etienne. Her work aims at developing and validating biophysical microenvironments able to replicate biological systems for tissue engineering and regenerative medicine applications.

Róisín M. Owens is Professor of Bioelectronics in the Department of Chemical Engineering and Biotechnology at the University of Cambridge and a Fellow of Newnham College. Her research centers on application of organic electronic materials for monitoring biological systems in vitro, with a specific interest in enhancing the biological complexity and adapting the electronics to be fit for purpose. She currently serves as co-investigator and co-director for the EPSRC CDT in Sensor Technologies, renewed in 2019. She is a 2019 laureate of the Suffrage Science award. In 2020, she became Scientific Editor for Materials Horizons (RSC). Her work has been cited more than 5000 times.

\section{ACKNOWLEDGMENTS}

R.O. acknowledges funding from the European Research Council (ERC) under the European Union's Horizon 2020 research and innovation program (Grant Agreement No. 723951). This material is also based upon work supported by the Air Force Office of Scientific Research under award number FA8655-20-1-7021 to RMO. A.M.P. acknowledges funding from the Oppenheimer Junior Research Fellowship and the Maudslay-Butler Research Fellowship at Pembroke College, Cambridge. D.v.N. is funded by the W.D Armstrong Trust Fund and the Oppenheimer Memorial Trust. Y.F. and D.I. were funded by the European Space Agency project BONUS. J.S. acknowledges funding from the European Union's Horizon 2020 research and innovation programme under the Marie Skłodowska-Curie grant, ICE METs (No. 842356). A.J.B. acknowledges support from his Cross-disciplinary Fellowship (Grant No. LT000034/2020-C) from the Human Frontier Science Program Organization. A.S. acknowledges funding from the European Union's Horizon 2020 research and innovation programme under the Marie Skłodowska-Curie grant, MultiStem (No. 895801).

\section{REFERENCES}

(1) Doke, S. K.; Dhawale, S. C. Alternatives to Animal Testing: A Review. Saudi Pharm. J. 2015, 23, 223-229.

(2) Graham, M. L.; Prescott, M. J. The Multifactorial Role of the 3Rs in Shifting the Harm-Benefit Analysis in Animal Models of Disease. Eur. J. Pharmacol. 2015, 759, 19-29.

(3) Aslantürk, Ö. S. In Vitro Cytotoxicity and Cell Viability Assays: Principles, Advantages, and Disadvantages. Genotoxicity - A Predict. Risk to Our Actual World 2018, 71923.

(4) Whitesides, G. M. The Origins and the Future of Microfluidics. Nature 2006, 442, 368-373.

(5) Lutolf, M. P.; Hubbell, J. A. Synthetic Biomaterials as Instructive Extracellular Microenvironments for Morphogenesis in Tissue Engineering. Nat. Biotechnol. 2005, 23, 47-55.
(6) Wu, Q.; Liu, J.; Wang, X.; Feng, L.; Wu, J.; Zhu, X.; Wen, W.; Gong, X. Organ-on-a-Chip: Recent Breakthroughs and Future Prospects. Biomed. Eng. Online 2020, 19, 9.

(7) Zhang, B.; Radisic, M. Organ-on-A-Chip Devices Advance to Market. Lab Chip 2017, 17, 2395-2420.

(8) Soucy, J. R.; Bindas, A. J.; Koppes, A. N.; Koppes, R. A. Instrumented Microphysiological Systems for Real-Time Measurement and Manipulation of Cellular Electrochemical Processes. iScience 2019, 21, 521-548.

(9) Arrowsmith, J.; Miller, P. Trial Watch: Phase II and Phase III Attrition Rates 2011-2012. Nat. Rev. Drug Discovery 2013, 12, 569.

(10) Khanna, I. Drug Discovery in Pharmaceutical Industry: Productivity Challenges and Trends. Drug Discovery Today 2012, 17, $1088-1102$

(11) Ronaldson-Bouchard, K.; Vunjak-Novakovic, G. Organs-on-aChip: A Fast Track for Engineered Human Tissues in Drug Development. Cell Stem Cell 2018, 22, 310-324.

(12) Ho, D.; Quake, S. R.; McCabe, E. R. B.; Chng, W. J.; Chow, E. K.; Ding, X.; Gelb, B. D.; Ginsburg, G. S.; Hassenstab, J.; Ho, C. M.; et al. Enabling Technologies for Personalized and Precision Medicine. Trends in Biotechnology. Elsevier Ltd May 1, 2020; pp 497-518.

(13) Ginsburg, G. S.; Phillips, K. A. Precision Medicine: From Science to Value. Health Aff. 2018, 37, 694-701.

(14) Langhans, S. A. Three-Dimensional in Vitro Cell Culture Models in Drug Discovery and Drug Repositioning. Front. Pharmacol. 2018, 9, 6.

(15) Denayer, T.; Stöhr, T.; Van Roy, M. Animal Models in Translational Medicine: Validation and Prediction. New Horizons Transl. Med. 2017, 2, 5-11.

(16) Moysidou, C. M.; Barberio, C.; Owens, R. M. Advances in Engineering Human Tissue Models. Front. Bioeng. Biotechnol. 2021, 8, 620962 .

(17) Sneddon, L. U.; Halsey, L. G.; Bury, N. R. Considering Aspects of the 3Rs Principles within Experimental Animal Biology. J. Exp. Biol. 2017, 220, 3007-3016.

(18) In Vitro Toxicity Testing Market - Global Forecast to 2025. MarketsandMarkets https://www.marketsandmarkets.com/MarketReports/in-vitro-toxicology-testing-market-209577065.html (accessed Apr 23, 2021).

(19) Sterilization Equipment Market by Product, End User - Global Forecast to 2025. MarketsandMarkets; 2021, https: / /www.reportlinker. $\mathrm{com} / \mathrm{p} 05099513 /$ Cell-Based-Assays-Market-By-Product-ApplicationEnd-User-Global-Forecast-to.html?utm_source=GNW.

(20) Organ-On-Chip (OOC) Market Size is USD 303.6 Million by 2026 at CAGR 39.9\%/Valuates Reports, https://www.prnewswire. $\mathrm{com} /$ in/news-releases/organ-on-chip-ooc-market-size-is-usd-303-6million-by-2026-at-cagr-39-9-valuates-reports-856995001.html (accessed Apr 23, 2021).

(21) Luca, A. C.; Mersch, S.; Deenen, R.; Schmidt, S.; Messner, I.; Schäfer, K. L.; Baldus, S. E.; Huckenbeck, W.; Piekorz, R. P.; Knoefel, W. T.; et al. Impact of the 3D Microenvironment on Phenotype, Gene Expression, and EGFR Inhibition of Colorectal Cancer Cell Lines. PLoS One 2013, 8, e59689.

(22) Fitzgerald, K. A.; Malhotra, M.; Curtin, C. M.; O’Brien, F. J.; O’Driscoll, C. M. Life in 3D Is Never Flat: 3D Models to Optimise Drug Delivery. J. Controlled Release 2015, 215, 39-54.

(23) Antoni, D.; Burckel, H.; Josset, E.; Noel, G. Three-Dimensional Cell Culture: A Breakthrough in Vivo. Int. J. Mol. Sci. 2015, 16, 55175527.

(24) Kieninger, J.; Weltin, A.; Flamm, H.; Urban, G. A. Microsensor Systems for Cell Metabolism-from 2D Culture to Organ-on-Chip. Lab Chip 2018, 18, 1274-1291.

(25) Lancaster, M. A.; Huch, M. Disease Modelling in Human Organoids. Dis. Models Mech. 2019, 12, dmm039347.

(26) Terrell, J. A.; Jones, C. G.; Kabandana, G. K. M.; Chen, C. From Cells-on-a-Chip to Organs-on-a-Chip: Scaffolding Materials for 3D Cell Culture in Microfluidics. J. Mater. Chem. B 2020, 8, 6667.

(27) Campbell, S. B.; Wu, Q.; Yazbeck, J.; Liu, C.; Okhovatian, S.; Radisic, M. Beyond Polydimethylsiloxane: Alternative Materials for 
Fabrication of Organ-on-a-chip Devices and Microphysiological Systems. ACS Biomater. Sci. Eng. 2021, 7, 2880.

(28) Giaever, I.; Keese, C. R. Micromotion of Mammalian Cells Measured Electrically. Proc. Natl. Acad. Sci. U. S. A. 1991, 88, 78967900.

(29) Pappa, A. M.; Parlak, O.; Scheiblin, G.; Mailley, P.; Salleo, A.; Owens, R. M. Organic Electronics for Point-of-Care Metabolite Monitoring. Trends Biotechnol. 2018, 36, 45-59.

(30) Thielecke, H.; Mack, A.; Robitzki, A. A Multicellular SpheroidBased Sensor for Anti-Cancer Therapeutics. Biosens. Bioelectron. 2001, 16, 261-269.

(31) McDonald, M.; Sebinger, D.; Brauns, L.; Gonzalez-Cano, L.; Menuchin-Lasowski, Y.; Psathaki, O. E.; Stumpf, A.; Rauen, T.; Schöler, H.; Jones, P. D. A Mesh Microelectrode Array for Non-Invasive Electrophysiology within Neural Organoids. bioRxiv 2020, DOI: $10.1101 / 2020.09 .02 .279125$.

(32) Kloß, D.; Kurz, R.; Jahnke, H. G.; Fischer, M.; Rothermel, A.; Anderegg, U.; Simon, J. C.; Robitzki, A. A. Microcavity Array (MCA)Based Biosensor Chip for Functional Drug Screening of 3D Tissue Models. Biosens. Bioelectron. 2008, 23, 1473-1480.

(33) Lei, K. F.; Lin, B. Y.; Tsang, N. M. Real-Time and Label-Free Impedimetric Analysis of the Formation and Drug Testing of Tumor Spheroids Formed via the Liquid Overlay Technique. RSC Adv. 2017, 7, 13939-13946.

(34) Lee, S. M.; Han, N.; Lee, R.; Choi, I. H.; Park, Y. B.; Shin, J. S.; Yoo, K. H. Real-Time Monitoring of 3D Cell Culture Using a 3D Capacitance Biosensor. Biosens. Bioelectron. 2016, 77, 56-61.

(35) Bürgel, S. C.; Diener, L.; Frey, O.; Kim, J. Y.; Hierlemann, A. Automated, Multiplexed Electrical Impedance Spectroscopy Platform for Continuous Monitoring of Microtissue Spheroids. Anal. Chem. 2016, 88, 10876-10883.

(36) Giaever, I.; Keese, C. R. Monitoring Fibroblast Behavior in Tissue Culture with an Applied Electric Field. Proc. Natl. Acad. Sci. U. S. A. 1984, 81, 3761-3764.

(37) Giaever, I.; Keese, C. R. A Morphological Biosensor for Mammalian Cells. Nature 1993, 366, 591-592.

(38) De León, S. E.; Pupovac, A.; McArthur, S. L. Three-Dimensional (3D) Cell Culture Monitoring: Opportunities and Challenges for Impedance Spectroscopy. Biotechnol. Bioeng. 2020, 117, 1230-1240.

(39) Jayaram, A. K.; Pitsalidis, C.; Tan, E.; Moysidou, C.-M.; De Volder, M. F. L.; Kim, J.-S.; Owens, R. M. 3D Hybrid Scaffolds Based on PEDOT:PSS/MWCNT Composites. Front. Chem. 2019, 7, 363.

(40) Moysidou, C. M.; Pitsalidis, C.; Al-Sharabi, M.; Withers, A. M.; Zeitler, J. A.; Owens, R. M. 3D Bioelectronic Model of the Human Intestine. Adv. Biol. 2021, 5, 2000306

(41) Inal, S.; Hama, A.; Ferro, M.; Pitsalidis, C.; Oziat, J.; Iandolo, D.; Pappa, A.-M.; Hadida, M.; Huerta, M.; Marchat, D.; et al. Conducting Polymer Scaffolds for Hosting and Monitoring 3D Cell Culture. Adv. Biosyst. 2017, 1, 1700052.

(42) Yang, R.; Tam, C. H.; Cheung, K. L.; Wong, K. C.; Xi, N.; Yang, J.; Lai, K. W. C. Cell Segmentation and Pipette Identification for Automated Patch Clamp Recording. Robot. Biomimetics 2014, 1, 20.

(43) Maher, M. P.; Pine, J.; Wright, J.; Tai, Y. C. The Neurochip: A New Multielectrode Device for Stimulating and Recording from Cultured Neurons. J. Neurosci. Methods 1999, 87, 45-56.

(44) Imboden, M.; de Coulon, E.; Poulin, A.; Dellenbach, C.; Rosset, S.; Shea, H.; Rohr, S. High-Speed Mechano-Active Multielectrode Array for Investigating Rapid Stretch Effects on Cardiac Tissue. Nat. Commun. 2019, 10, 834.

(45) Yuan, X.; Schröter, M.; Obien, M. E. J.; Fiscella, M.; Gong, W.; Kikuchi, T.; Odawara, A.; Noji, S.; Suzuki, I.; Takahashi, J.; et al. Versatile Live-Cell Activity Analysis Platform for Characterization of Neuronal Dynamics at Single-Cell and Network Level. Nat. Commun. 2020, 11, 4854.

(46) Zhang, X.; Wang, W.; Nordin, A. N.; Li, F.; Jang, S.; Voiculescu, I. The Influence of the Electrode Dimension on the Detection Sensitivity of Electric Cell-Substrate Impedance Sensing (ECIS) and Its Mathematical Modeling. Sens. Actuators, B 2017, 247, 780-790.
(47) Liu, H.; Bolonduro, O. A.; Hu, N.; Ju, J.; Rao, A. A.; Duffy, B. M.; Huang, Z.; Black, L. D.; Timko, B. P. Heart-on-a-Chip Model with Integrated Extra- and Intracellular Bioelectronics for Monitoring Cardiac Electrophysiology under Acute Hypoxia. Nano Lett. 2020, 20, 2585-2593.

(48) van der Helm, M. W.; Henry, O. Y. F.; Bein, A.; Hamkins-Indik, T.; Cronce, M. J.; Leineweber, W. D.; Odijk, M.; van der Meer, A. D.; Segerink, L. I.; Ingber, D. E.; et al. Non-Invasive Sensing of Transepithelial Barrier Function and Tissue Differentiation in Organs-on-Chips Using Impedance Spectroscopy. Lab Chip 2019, 19, 452-463.

(49) Yan, Z.; Han, M.; Shi, Y.; Badea, A.; Yang, Y.; Kulkarni, A.; Hanson, E.; Kandel, M. E.; Wen, X.; Zhang, F.; et al. ThreeDimensional Mesostructures as High-Temperature Growth Templates, Electronic Cellular Scaffolds, and Self-Propelled Microrobots. Proc. Natl. Acad. Sci. U. S. A. 2017, 114, E9455-E9464.

(50) Wan, A. M. D.; Inal, S.; Williams, T.; Wang, K.; Leleux, P.; Estevez, L.; Giannelis, E. P.; Fischbach, C.; Malliaras, G. G.; Gourdon, D. 3D Conducting Polymer Platforms for Electrical Control of Protein Conformation and Cellular Functions. J. Mater. Chem. B 2015, 3, 5040-5048.

(51) Guex, A. G.; Puetzer, J. L.; Armgarth, A.; Littmann, E.; Stavrinidou, E.; Giannelis, E. P.; Malliaras, G. G.; Stevens, M. M. Highly Porous Scaffolds of PEDOT:PSS for Bone Tissue Engineering. Acta Biomater. 2017, 62, 91-101.

(52) Iandolo, D.; Sheard, J.; Karavitas Levy, G.; Pitsalidis, C.; Tan, E.; Dennis, A.; Kim, J. S.; Markaki, A. E.; Widera, D.; Owens, R. M. Biomimetic and Electroactive 3D Scaffolds for Human Neural CrestDerived Stem Cell Expansion and Osteogenic Differentiation. MRS Commun. 2020, 10, 179-187.

(53) Pitsalidis, C.; Ferro, M. P.; Iandolo, D.; Tzounis, L.; Inal, S.; Owens, R. M. Transistor in a Tube: A Route to Three-Dimensional Bioelectronics. Sci. Adv. 2018, 4, eaat 4253.

(54) Pitsalidis, C.; Pappa, A.-M.; Hunter, S.; Payne, M. M.; Anthony, J. E.; Anthopoulos, T. D.; Logothetidis, S. Electrospray-Processed Soluble Acenes toward the Realization of High-Performance FieldEffect Transistors. ACS Appl. Mater. Interfaces 2015, 7, 6496.

(55) Inal, S.; Rivnay, J.; Suiu, A. O.; Malliaras, G. G.; McCulloch, I. Conjugated Polymers in Bioelectronics. Acc. Chem. Res. 2018, 51, $1368-1376$.

(56) Benfenati, V.; Toffanin, S.; Bonetti, S.; Turatti, G.; Pistone, A.; Chiappalone, M.; Sagnella, A.; Stefani, A.; Generali, G.; Ruani, G.; et al. A Transparent Organic Transistor Structure for Bidirectional Stimulation and Recording of Primary Neurons. Nat. Mater. 2013, $12,672-680$.

(57) Spanu, A.; Lai, S.; Cosseddu, P.; Tedesco, M.; Martinoia, S.; Bonfiglio, A. An Organic Transistor-Based System for Reference-Less Electrophysiological Monitoring of Excitable Cells. Sci. Rep. 2015, 5, 8807.

(58) Yao, C.; Li, Q.; Guo, J.; Yan, F.; Hsing, I. M. Rigid and Flexible Organic Electrochemical Transistor Arrays for Monitoring Action Potentials from Electrogenic Cells. Adv. Healthcare Mater. 2015, 4, $528-533$.

(59) Huerta, M.; Rivnay, J.; Ramuz, M.; Hama, A.; Owens, R. M. Early Detection of Nephrotoxicity In Vitro Using a Transparent Conducting Polymer Device. Appl. Vitr. Toxicol. 2016, 2, 17-25.

(60) Rivnay, J.; Ramuz, M.; Leleux, P.; Hama, A.; Huerta, M.; Owens, R. M. Organic Electrochemical Transistors for Cell-Based Impedance Sensing. Appl. Phys. Lett. 2015, 106, 043301.

(61) Higgins, S. G.; Lo Fiego, A.; Patrick, I.; Creamer, A.; Stevens, M. M. Organic Bioelectronics: Using Highly Conjugated Polymers to Interface with Biomolecules, Cells, and Tissues in the Human Body. Adv. Mater. Technol. 2020, 5, 2000384.

(62) Ohayon, D.; Inal, S. Organic Bioelectronics: From Functional Materials to Next-Generation Devices and Power Sources. Adv. Mater. 2020, 32, 2001439.

(63) Sundelacruz, S.; Li, C.; Choi, Y. J.; Levin, M.; Kaplan, D. L. Bioelectric Modulation of Wound Healing in a 3D Invitro Model of Tissue-Engineered Bone. Biomaterials 2013, 34, 6695-6705. 
(64) Simon, D. T.; Gabrielsson, E. O.; Tybrandt, K.; Berggren, M. Organic Bioelectronics: Bridging the Signaling Gap between Biology and Technology. Chem. Rev. 2016, 116, 13009-13041.

(65) Schmidt, C. E.; Shastri, V. R.; Vacanti, J. P.; Langer, R. Stimulation of Neurite Outgrowth Using an Electrically Conducting Polymer. Proc. Natl. Acad. Sci. U. S. A. 1997, 94, 8948-8953.

(66) Gelmi, A.; Schutt, C. E. Stimuli-Responsive Biomaterials: Scaffolds for Stem Cell Control. Adv. Healthcare Mater. 2021, 10, 2001125 .

(67) Löffler, S.; Libberton, B.; Richter-Dahlfors, A. Organic Bioelectronic Tools for Biomedical Applications. Electronics 2015, 4, 879-908.

(68) Dijk, G.; Rutz, A. L.; Malliaras, G. G. Stability of PEDOT:PSSCoated Gold Electrodes in Cell Culture Conditions. Adv. Mater. Technol. 2020, 5, 1900662.

(69) Le, T. H.; Kim, Y.; Yoon, H. Electrical and Electrochemical Properties of Conducting Polymers. Polymers (Basel, Switz.) 2017, 9, 150.

(70) Epstein, A. J. Electrical Conductivity in Conjugated Polymers. Annu. Technol. Conf. - ANTEC, Conf. Proc. 1998, 2, 1248-1252.

(71) Vezie, M. S.; Few, S.; Meager, I.; Pieridou, G.; Dörling, B.; Ashraf, R. S.; Goñi, A. R.; Bronstein, H.; McCulloch, I.; Hayes, S. C.; et al. Exploring the Origin of High Optical Absorption in Conjugated Polymers. Nat. Mater. 2016, 15, 746-753.

(72) Jozefowicz, M.; Yu, L. T.; Belorgey, G.; Buvet, R. Conductivité Electronique et Propriétés Chimiques de Polyanilines Oligomères. J. Polym. Sci., Part C: Polym. Symp. 1967, 16, 2943-2954.

(73) Macdiarmid, A. G.; Chiang, J. C.; Richter, A. F.; Epstein, A. J. Polyaniline: A New Concept in Conducting Polymers. Synth. Met. 1987, 18, 285-290.

(74) Yurash, B.; Cao, D. X.; Brus, V. V.; Leifert, D.; Wang, M.; Dixon, A.; Seifrid, M.; Mansour, A. E.; Lungwitz, D.; Liu, T.; et al. Towards Understanding the Doping Mechanism of Organic Semiconductors by Lewis Acids. Nat. Mater. 2019, 18, 1327-1334.

(75) Li, H.; DeCoster, M. E.; Ming, C.; Wang, M.; Chen, Y.; Hopkins, P. E.; Chen, L.; Katz, H. E. Enhanced Molecular Doping for High Conductivity in Polymers with Volume Freed for Dopants. Macromolecules 2019, 52, 9804-9812.

(76) Keene, S. T.; van der Pol, T. P. A.; Zakhidov, D.; Weijtens, C. H. L.; Janssen, R. A. J.; Salleo, A.; van de Burgt, Y. Enhancement-Mode PEDOT:PSS Organic Electrochemical Transistors Using Molecular De-Doping. Adv. Mater. 2020, 32, 2000270.

(77) Rivnay, J.; Inal, S.; Collins, B. A.; Sessolo, M.; Stavrinidou, E.; Strakosas, X.; Tassone, C.; Delongchamp, D. M.; Malliaras, G. G. Structural Control of Mixed Ionic and Electronic Transport in Conducting Polymers. Nat. Commun. 2016, 7, 11287.

(78) Zhang, S.; Li, Y.; Tomasello, G.; Anthonisen, M.; Li, X.; Mazzeo, M.; Genco, A.; Grutter, P.; Cicoira, F. Tuning the Electromechanical Properties of PEDOT:PSS Films for Stretchable Transistors And Pressure Sensors. Adv. Electron. Mater. 2019, 5, 1900191.

(79) Anderson, J. M. Biocompatibility and the Relationship to Standards: Meaning and Scope of Biomaterials Testing. Compr. Biomater. 2011, 4, 7-26.

(80) Boehler, C.; Oberueber, F.; Schlabach, S.; Stieglitz, T.; Asplund, M. Long-Term Stable Adhesion for Conducting Polymers in Biomedical Applications: IrOx and Nanostructured Platinum Solve the Chronic Challenge. ACS Appl. Mater. Interfaces 2017, 9, 189-197.

(81) Wustoni, S.; Saleh, A.; El-Demellawi, J. K.; Koklu, A.; Hama, A.; Druet, V.; Wehbe, N.; Zhang, Y.; Inal, S. MXene Improves the Stability and Electrochemical Performance of Electropolymerized PEDOT Films. APL Mater. 2020, 8, 121105.

(82) Berggren, M.; Crispin, X.; Fabiano, S.; Jonsson, M. P.; Simon, D. T.; Stavrinidou, E.; Tybrandt, K.; Zozoulenko, I. Ion Electron-Coupled Functionality in Materials and Devices Based on Conjugated Polymers. Adv. Mater. 2019, 31, 1805813

(83) Savva, A.; Hallani, R.; Cendra, C.; Surgailis, J.; Hidalgo, T. C.; Wustoni, S.; Sheelamanthula, R.; Chen, X.; Kirkus, M.; Giovannitti, A.; et al. Balancing Ionic and Electronic Conduction for High-Performance
Organic Electrochemical Transistors. Adv. Funct. Mater. 2020, 30, 1907657.

(84) Leleux, P.; Badier, J.-M.; Rivnay, J.; Bénar, C.; Hervé, T.; Chauvel, P.; Malliaras, G. G. Conducting Polymer Electrodes for Electroencephalography. Adv. Healthcare Mater. 2014, 3, 490-493.

(85) Akhtar, R.; Sherratt, M. J.; Cruickshank, J. K.; Derby, B. Characterizing the Elastic Properties of Tissues. Mater. Today 2011, 14, 96-105.

(86) Qu, J.; Ouyang, L.; Kuo, C. C.; Martin, D. C. Stiffness, Strength and Adhesion Characterization of Electrochemically Deposited Conjugated Polymer Films. Acta Biomater. 2016, 31, 114-121.

(87) Rivnay, J.; Wang, H.; Fenno, L.; Deisseroth, K.; Malliaras, G. G. Next-Generation Probes, Particles, and Proteins for Neural Interfacing. Sci. Adv. 2017, e1601649.

(88) Fu, F.; Wang, J.; Zeng, H.; Yu, J. Functional Conductive Hydrogels for Bioelectronics. ACS Mater. Lett. 2020, 2, 1287-1301.

(89) Tomczykowa, M.; Plonska-Brzezinska, M. E. Conducting Polymers, Hydrogels and Their Composites: Preparation, Properties and Bioapplications. Polymers (Basel, Switz.) 2019, 11, 350.

(90) Balint, R.; Cassidy, N. J.; Cartmell, S. H. Conductive Polymers: Towards a Smart Biomaterial for Tissue Engineering. Acta Biomater. 2014, 10, 2341-2353.

(91) Wang, S.; Sun, C.; Guan, S.; Li, W.; Xu, J.; Ge, D.; Zhuang, M.; Liu, T.; Ma, X. Chitosan/Gelatin Porous Scaffolds Assembled with Conductive Poly(3,4-Ethylenedioxythiophene) Nanoparticles for Neural Tissue Engineering. J. Mater. Chem. B 2017, 5, 4774-4788.

(92) Wang, S.; Guan, S.; Zhu, Z.; Li, W.; Liu, T.; Ma, X. Hyaluronic Acid Doped-Poly(3,4-Ethylenedioxythiophene)/Chitosan/Gelatin (PEDOT-HA/Cs/Gel) Porous Conductive Scaffold for Nerve Regeneration. Mater. Sci. Eng., C 2017, 71, 308-316.

(93) Li, M.; Guo, Y.; Wei, Y.; MacDiarmid, A. G.; Lelkes, P. I. Electrospinning Polyaniline-Contained Gelatin Nanofibers for Tissue Engineering Applications. Biomaterials 2006, 27, 2705-2715.

(94) Moutsatsou, P.; Coopman, K.; Georgiadou, S. Biocompatibility Assessment of Conducting PANI/Chitosan Nanofibers for Wound Healing Applications. Polymers (Basel, Switz.) 2017, 9, 687.

(95) Mihic, A.; Cui, Z.; Wu, J.; Vlacic, G.; Miyagi, Y.; Li, S.-H.; Lu, S.; Sung, H.-W.; Weisel, R. D.; Li, R.-K. A Conductive Polymer Hydrogel Supports Cell Electrical Signaling and Improves Cardiac Function After Implantation into Myocardial Infarct. Circulation 2015, 132, 772-784.

(96) Mao, J.; Zhang, Z. Polypyrrole as Electrically Conductive Biomaterials: Synthesis, Biofunctionalization, Potential Applications and Challenges. In Advances in Experimental Medicine and Biology; Chun, H. J., Park, C. H., Kwon, I. K., Khang, G., Eds.; Springer Singapore: Singapore, 2018; Vol. 1078, pp 347-370.

(97) Yang, J.; Choe, G.; Yang, S.; Jo, H.; Lee, J. Y. PolypyrroleIncorporated Conductive Hyaluronic Acid Hydrogels. Biomater. Res. 2016, 20, 31.

(98) Richardson, R. T.; Thompson, B.; Moulton, S.; Newbold, C.; Lum, M. G.; Cameron, A.; Wallace, G.; Kapsa, R.; Clark, G.; O’Leary, S. The Effect of Polypyrrole with Incorporated Neurotrophin-3 on the Promotion of Neurite Outgrowth from Auditory Neurons. Biomaterials 2007, 28, 513-523.

(99) Shin, J.; Choi, E. J.; Cho, J. H.; Cho, A.-N.; Jin, Y.; Yang, K.; Song, C.; Cho, S.-W. Three-Dimensional Electroconductive Hyaluronic Acid Hydrogels Incorporated with Carbon Nanotubes and Polypyrrole by Catechol-Mediated Dispersion Enhance Neurogenesis of Human Neural Stem Cells. Biomacromolecules 2017, 18, 3060-3072.

(100) Boehler, C.; Aqrawe, Z.; Asplund, M. Applications of PEDOT in Bioelectronic Medicine. Bioelectron. Med. 2019, 2, 89-99.

(101) Donahue, M. J.; Proctor, C. M.; Strakosas, X. Chapter 13 Polymers/PEDOT Derivatives for Bioelectronics. Redox Polymers for Energy and Nanomedicine; The Royal Society of Chemistry, 2021; pp $488-545$

(102) Zozoulenko, I.; Singh, A.; Singh, S. K.; Gueskine, V.; Crispin, X.; Berggren, M. Polarons, Bipolarons, And Absorption Spectroscopy of PEDOT. ACS Appl. Polym. Mater. 2019, 1, 83-94.

(103) Stavrinidou, E.; Leleux, P.; Rajaona, H.; Khodagholy, D.; Rivnay, J.; Lindau, M.; Sanaur, S.; Malliaras, G. G. Direct Measurement 
of Ion Mobility in a Conducting Polymer. Adv. Mater. 2013, 25, 44884493.

(104) Stavrinidou, E.; Leleux, P.; Rajaona, H.; Fiocchi, M.; Sanaur, S.; Malliaras, G. G. A Simple Model for Ion Injection and Transport in Conducting Polymers. J. Appl. Phys. 2013, 113, 244501.

(105) Savva, A.; Wustoni, S.; Inal, S. Ionic-to-Electronic Coupling Efficiency in PEDOT:PSS Films Operated in Aqueous Electrolytes. J. Mater. Chem. C 2018, 6, 12023-12030.

(106) Proctor, C. M.; Rivnay, J.; Malliaras, G. G. Understanding Volumetric Capacitance in Conducting Polymers. J. Polym. Sci., Part B: Polym. Phys. 2016, 54, 1433-1436.

(107) Berggren, M.; Malliaras, G. G. How Conducting Polymer Electrodes Operate. Science (Washington, DC, U. S.) 2019, 364, 233234.

(108) Romele, P.; Ghittorelli, M.; Kovács-Vajna, Z. M.; Torricelli, F. Ion buffering and interface charge enable high performance electronics with organic electrochemical transistors. Nat. Commun. 2019, 10, No. 3044.

(109) Rivnay, J.; Leleux, P.; Ferro, M.; Sessolo, M.; Williamson, A.; Koutsouras, D. A.; Khodagholy, D.; Ramuz, M.; Strakosas, X.; Owens, R. M.; et al. High-Performance Transistors for Bioelectronics through Tuning of Channel Thickness. Sci. Adv. 2015, 1, e1400251.

(110) Volkov, A. V.; Wijeratne, K.; Mitraka, E.; Ail, U.; Zhao, D.; Tybrandt, K.; Andreasen, J. W.; Berggren, M.; Crispin, X.; Zozoulenko, I. V. Understanding the Capacitance of PEDOT:PSS. Adv. Funct. Mater. 2017, 27, 1700329.

(111) Modarresi, M.; Mehandzhiyski, A.; Fahlman, M.; Tybrandt, K.; Zozoulenko, I. Microscopic Understanding of the Granular Structure and the Swelling of PEDOT:PSS. Macromolecules 2020, 53, 62676278

(112) Savva, A.; Wustoni, S.; Inal, S. Ionic-to-Electronic Coupling Efficiency in PEDOT:PSS Films Operated in Aqueous Electrolytes. $J$. Mater. Chem. C 2018, 6, 12023-12030.

(113) Solazzo, M.; Krukiewicz, K.; Zhussupbekova, A.; Fleischer, K.; Biggs, M. J.; Monaghan, M. G. PEDOT:PSS Interfaces Stabilised Using a PEGylated Crosslinker Yield Improved Conductivity and Biocompatibility. J. Mater. Chem. B 2019, 7, 4811-4820.

(114) Håkansson, A.; Han, S.; Wang, S.; Lu, J.; Braun, S.; Fahlman, M.; Berggren, M.; Crispin, X.; Fabiano, S. Effect of (3Glycidyloxypropyl)Trimethoxysilane (GOPS) on the Electrical Properties of PEDOT:PSS Films. J. Polym. Sci., Part B: Polym. Phys. 2017, 55, 814-820.

(115) El Mahmoudy, M.; Inal, S.; Charrier, A.; Uguz, I.; Malliaras, G. G.; Sanaur, S. Tailoring the Electrochemical and Mechanical Properties of PEDOT:PSS Films for Bioelectronics. Macromol. Mater. Eng. 2017, 302, 1600497.

(116) Mantione, D.; Del Agua, I.; Schaafsma, W.; Elmahmoudy, M.; Uguz, I.; Sanchez-Sanchez, A.; Sardon, H.; Castro, B.; Malliaras, G. G.; Mecerreyes, D. Low-Temperature Cross-Linking of PEDOT:PSS Films Using Divinylsulfone. ACS Appl. Mater. Interfaces 2017, 9, 1825418262 .

(117) del Agua, I.; Marina, S.; Pitsalidis, C.; Mantione, D.; Ferro, M.; Iandolo, D.; Sanchez-Sanchez, A.; Malliaras, G. G.; Owens, R. M.; Mecerreyes, D. Conducting Polymer Scaffolds Based on Poly(3,4Ethylenedioxythiophene) and Xanthan Gum for Live-Cell Monitoring. ACS Omega 2018, 3, 7424-7431.

(118) del Agua, I.; Mantione, D.; Ismailov, U.; Sanchez-Sanchez, A.; Aramburu, N.; Malliaras, G. G.; Mecerreyes, D.; Ismailova, E. DVSCrosslinked PEDOT:PSS Free-Standing and Textile Electrodes toward Wearable Health Monitoring. Adv. Mater. Technol. 2018, 3, 1700322.

(119) Kim, S.-M.; Kim, N.; Kim, Y.; Baik, M.-S.; Yoo, M.; Kim, D.; Lee, W.-J.; Kang, D.-H.; Kim, S.; Lee, K.; et al. High-Performance, Polymer-Based Direct Cellular Interfaces for Electrical Stimulation and Recording. NPG Asia Mater. 2018, 10, 255-265.

(120) Nikiforidis, G.; Wustoni, S.; Routier, C.; Hama, A.; Koklu, A.; Saleh, A.; Steiner, N.; Druet, V.; Fiumelli, H.; Inal, S. Benchmarking the Performance of Electropolymerized Poly(3,4-Ethylenedioxythiophene) Electrodes for Neural Interfacing. Macromol. Biosci. 2020, 20, 2000215.
(121) Borges-González, J.; Kousseff, C. J.; Nielsen, C. B. Organic Semiconductors for Biological Sensing. J. Mater. Chem. C 2019, 7, $1111-1130$.

(122) Moser, M.; Ponder, J. F., Jr.; Wadsworth, A.; Giovannitti, A.; McCulloch, I. Materials in Organic Electrochemical Transistors for Bioelectronic Applications: Past, Present, and Future. Adv. Funct. Mater. 2019, 29, 1807033.

(123) Inal, S.; Malliaras, G. G.; Rivnay, J. Benchmarking Organic Mixed Conductors for Transistors. Nat. Commun. 2017, 8, 1767.

(124) Paulsen, B. D.; Tybrandt, K.; Stavrinidou, E.; Rivnay, J. Organic Mixed Ionic-Electronic Conductors. Nat. Mater. 2020, 19, 13-26.

(125) Giovannitti, A.; Maria, I. P.; Hanifi, D.; Donahue, M. J.; Bryant, D.; Barth, K. J.; Makdah, B. E.; Savva, A.; Moia, D.; Zetek, M.; et al. The Role of the Side Chain on the Performance of N-Type Conjugated Polymers in Aqueous Electrolytes. Chem. Mater. 2018, 30, 2945.

(126) Moser, M.; Savagian, L. R.; Savva, A.; Matta, M.; Ponder, J. F.; Hidalgo, T. C.; Ohayon, D.; Hallani, R.; Reisjalali, M.; Troisi, A.; et al. Ethylene Glycol-Based Side Chain Length Engineering in Polythiophenes and Its Impact on Organic Electrochemical Transistor Performance. Chem. Mater. 2020, 32, 6618-6628.

(127) Schmode, P.; Savva, A.; Kahl, R.; Ohayon, D.; Meichsner, F.; Dolynchuk, O.; Thurn-Albrecht, T.; Inal, S.; Thelakkat, M. The Key Role of Side Chain Linkage in Structure Formation and Mixed Conduction of Ethylene Glycol Substituted Polythiophenes. ACS Appl. Mater. Interfaces 2020, 12, 13029-13039.

(128) Savva, A.; Cendra, C.; Giugni, A.; Torre, B.; Surgailis, J.; Ohayon, D.; Giovannitti, A.; McCulloch, I.; Di Fabrizio, E.; Salleo, A.; et al. Influence of Water on the Performance of Organic Electrochemical Transistors. Chem. Mater. 2019, 31, 927-937.

(129) Giovannitti, A.; Sbircea, D.-T.; Inal, S.; Nielsen, C. B.; Bandiello, E.; Hanifi, D. A.; Sessolo, M.; Malliaras, G. G.; McCulloch, I.; Rivnay, J. Controlling the Mode of Operation of Organic Transistors through Side-Chain Engineering. Proc. Natl. Acad. Sci. U. S. A. 2016, 113, 12017-12022.

(130) Onorato, J. W.; Luscombe, C. K. Morphological Effects on Polymeric Mixed Ionic/Electronic Conductors. Mol. Syst. Des. Eng. 2019, 4, 310-324.

(131) Surgailis, J.; Savva, A.; Druet, V.; Paulsen, B. D.; Wu, R.; Hamidi-Sakr, A.; Ohayon, D.; Nikiforidis, G.; Chen, X.; McCulloch, I.; et al. Mixed Conduction in an N-Type Organic Semiconductor in the Absence of Hydrophilic Side-Chains. Adv. Funct. Mater. 2021, 31, 2010165.

(132) Nielsen, C. B.; Giovannitti, A.; Sbircea, D. T.; Bandiello, E.; Niazi, M. R.; Hanifi, D. A.; Sessolo, M.; Amassian, A.; Malliaras, G. G.; Rivnay, J.; et al. Molecular Design of Semiconducting Polymers for High-Performance Organic Electrochemical Transistors. J. Am. Chem. Soc. 2016, 138, 10252-10259.

(133) Ohayon, D.; Savva, A.; Du, W.; Paulsen, B. D.; Uguz, I.; Ashraf, R. S.; Rivnay, J.; McCulloch, I.; Inal, S. Influence of Side Chains on the N-Type Organic Electrochemical Transistor Performance. ACS Appl. Mater. Interfaces 2021, 13, 4253-4266.

(134) Ghezzi, D.; Antognazza, M. R.; Dal Maschio, M.; Lanzarini, E.; Benfenati, F.; Lanzani, G. A Hybrid Bioorganic Interface for Neuronal Photoactivation. Nat. Commun. 2011, 2, 166.

(135) Macchia, E.; Manoli, K.; Holzer, B.; Di Franco, C.; Ghittorelli, M.; Torricelli, F.; Alberga, D.; Mangiatordi, G. F.; Palazzo, G.; Scamarcio, G.; Torsi, L. Single-Molecule Detection with a MillimetreSized Transistor. Nat. Commun. 2018, 9, 3223.

(136) Inal, S.; Rivnay, J.; Leleux, P.; Ferro, M.; Ramuz, M.; Brendel, J. C.; Schmidt, M. M.; Thelakkat, M.; Malliaras, G. G. A High Transconductance Accumulation Mode Electrochemical Transistor. Adv. Mater. 2014, 26, 7450-7455.

(137) Nicolini, T.; Surgailis, J.; Savva, A.; Scaccabarozzi, A. D.; Nakar, R.; Thuau, D.; Wantz, G.; Richter, L. J.; Dautel, O.; Hadziioannou, G.; et al. A Low-Swelling Polymeric Mixed Conductor Operating in Aqueous Electrolytes. Adv. Mater. 2021, 33, 2005723.

(138) Sun, H.; Vagin, M.; Wang, S.; Crispin, X.; Forchheimer, R.; Berggren, M.; Fabiano, S. Complementary Logic Circuits Based on 
High-Performance n-Type Organic Electrochemical Transistors. Adv. Mater. 2018, 30, 1704916.

(139) Moser, M.; Savva, A.; Thorley, K.; Paulsen, B. D.; Hidalgo, T. C.; Ohayon, D.; Chen, H.; Giovannitti, A.; Marks, A.; Gasparini, N.; et al. Polaron Delocalization in Donor-Acceptor Polymers and Its Impact on Organic Electrochemical Transistor Performance. Angew. Chem., Int. Ed. 2021, 60, 7777-7785.

(140) Du, W.; Ohayon, D.; Combe, C.; Mottier, L.; Maria, I. P.; Ashraf, R. S.; Fiumelli, H.; Inal, S.; McCulloch, I. Improving the Compatibility of Diketopyrrolopyrrole Semiconducting Polymers for Biological Interfacing by Lysine Attachment. Chem. Mater. 2018, 30, 6164-6172.

(141) Giovannitti, A.; Nielsen, C. B.; Sbircea, D.-T.; Inal, S.; Donahue, M.; Niazi, M. R.; Hanifi, D. A.; Amassian, A.; Malliaras, G. G.; Rivnay, J.; McCulloch, I. N-Type Organic Electrochemical Transistors with Stability in Water. Nat. Commun. 2016, 7, 13066.

(142) Di Pietro, R.; Fazzi, D.; Kehoe, T. B.; Sirringhaus, H. Spectroscopic Investigation of Oxygen- and Water-Induced Electron Trapping and Charge Transport Instabilities in n-Type Polymer Semiconductors. J. Am. Chem. Soc. 2012, 134, 14877-14889.

(143) Paterson, A. F.; Savva, A.; Wustoni, S.; Tsetseris, L.; Paulsen, B. D.; Faber, H.; Emwas, A. H.; Chen, X.; Nikiforidis, G.; Hidalgo, T. C.; et al. Water Stable Molecular N-Doping Produces Organic Electrochemical Transistors with High Transconductance and Record Stability. Nat. Commun. 2020, 11, 3004.

(144) Yang, C. Y.; Stoeckel, M. A.; Ruoko, T. P.; Wu, H. Y.; Liu, X.; Kolhe, N. B.; Wu, Z.; Puttisong, Y.; Musumeci, C.; Massetti, M.; et al. A High-Conductivity n-Type Polymeric Ink for Printed Electronics. Nat. Commun. 2021, 12, 2354.

(145) Romele, P.; Gkoupidenis, P.; Koutsouras, D. A.; Lieberth, K.; Kovács-Vajna, Z. M.; Blom, P. W. M.; Torricelli, F. Multiscale Real Time and High Sensitivity Ion Detection with Complementary Organic Electrochemical Transistors Amplifier. Nat. Commun. 2020, 11, 3743.

(146) Ohayon, D.; Nikiforidis, G.; Savva, A.; Giugni, A.; Wustoni, S.; Palanisamy, T.; Chen, X.; Maria, I. P.; Di Fabrizio, E.; Costa, P. M. F. J.; et al. Biofuel Powered Glucose Detection in Bodily Fluids with an NType Conjugated Polymer. Nat. Mater. 2020, 19, 456-463.

(147) Pappa, A. M.; Ohayon, D.; Giovannitti, A.; Maria, I. P.; Savva, A.; Uguz, I.; Rivnay, J.; McCulloch, I.; Owens, R. M.; Inal, S. Direct Metabolite Detection with an N-Type Accumulation Mode Organic Electrochemical Transistor. Sci. Adv. 2018, 4, eaat0911.

(148) Savva, A.; Ohayon, D.; Surgailis, J.; Paterson, A. F.; Hidalgo, T. C.; Chen, X.; Maria, I. P.; Paulsen, B. D.; Petty, A. J.; Rivnay, J.; et al. Solvent Engineering for High-Performance n-Type Organic Electrochemical Transistors. Adv. Electron. Mater. 2019, 5, 1900249.

(149) Bischak, C. G.; Flagg, L. Q.; Yan, K.; Li, C.-Z.; Ginger, D. S. Fullerene Active Layers for N-Type Organic Electrochemical Transistors. ACS Appl. Mater. Interfaces 2019, 11, 28138-28144.

(150) Wong, J. Y.; Langer, R.; Ingber, D. E. Electrically Conducting Polymers Can Noninvasively Control the Shape and Growth of Mammalian Cells. Proc. Natl. Acad. Sci. U. S. A. 1994, 91, 3201-3204.

(151) Gomez, N.; Schmidt, C. E. Nerve Growth Factor-Immobilized Polypyrrole: Bioactive Electrically Conducting Polymer for Enhanced Neurite Extension. J. Biomed. Mater. Res., Part A 2007, 81A, 135-149.

(152) Schmidt, C. E.; Shastri, V. R.; Vacanti, J. P.; Langer, R. Stimulation of Neurite Outgrowth Using an Electrically Conducting Polymer. Proc. Natl. Acad. Sci. U. S. A. 1997, 94, 8948-8953.

(153) Collazos-Castro, J. E.; Polo, J. L.; Hernández-Labrado, G. R.; Padial-Cañete, V.; García-Rama, C. Bioelectrochemical Control of Neural Cell Development on Conducting Polymers. Biomaterials 2010, 31, 9244-9255.

(154) Zhu, B.; Luo, S. C.; Zhao, H.; Lin, H. A.; Sekine, J.; Nakao, A.; Chen, C.; Yamashita, Y.; Yu, H. H. Large Enhancement in Neurite Outgrowth on a Cell Membrane-Mimicking Conducting Polymer. Nat. Commun. 2014, 5, 4523.

(155) Formentín, P.; Catalán; Pol, L.; Fernández-Castillejo, S.; Solà, R.; Marsal, L. F. Collagen and Fibronectin Surface Modification of Nanoporous Anodic Alumina and Macroporous Silicon for Endothelial Cell Cultures. J. Biol. Eng. 2018, 12, 21.
(156) Wei, Y.; Zhang, J.; Li, H.; Zhang, L.; Bi, H. Multifunctional Copolymer Coating of Polyethylene Glycol, Glycidyl Methacrylate, and REDV to Enhance the Selectivity of Endothelial Cells. J. Biomater. Sci., Polym. Ed. 2015, 26, 1357-1371.

(157) Fabregat, G.; Ballano, G.; Armelin, E.; Del Valle, L. J.; Cativiela, C.; Alemán, C. An Electroactive and Biologically Responsive Hybrid Conjugate Based on Chemical Similarity. Polym. Chem. 2013, 4, 14121424.

(158) Chen, L.; Yan, C.; Zheng, Z. Functional Polymer Surfaces for Controlling Cell Behaviors. Mater. Today 2018, 21, 38-59.

(159) Liu, X.; Yue, Z.; Higgins, M. J.; Wallace, G. G. Conducting Polymers with Immobilised Fibrillar Collagen for Enhanced Neural Interfacing. Biomaterials 2011, 32, 7309-7317.

(160) Molino, P. J.; Higgins, M. J.; Innis, P. C.; Kapsa, R. M. I.; Wallace, G. G. Fibronectin and Bovine Serum Albumin Adsorption and Conformational Dynamics on Inherently Conducting Polymers: A QCM-D Study. Langmuir 2012, 28, 8433-8445.

(161) Kotwal, A.; Schmidt, C. E. Electrical Stimulation Alters Protein Adsorption and Nerve Cell Interactions with Electrically Conducting Biomaterials. Biomaterials 2001, 22, 1055-1064.

(162) Sordini, L.; Garrudo, F. F. F.; Rodrigues, C. A. V.; Linhardt, R. J.; Cabral, J. M. S.; Ferreira, F. C.; Morgado, J. Effect of Electrical Stimulation Conditions on Neural Stem Cells Differentiation on CrossLinked PEDOT:PSS Films. Front. Bioeng. Biotechnol. 2021, 9, 591838.

(163) Bhagwat, N.; Murray, R. E.; Shah, S. I.; Kiick, K. L.; Martin, D. C. Biofunctionalization of PEDOT Films with Laminin-Derived Peptides. Acta Biomater. 2016, 41, 235-246.

(164) Darmanin, T.; Guittard, F. Wettability of Conducting Polymers: From Superhydrophilicity to Superoleophobicity. Prog. Polym. Sci. 2014, 39, 656-682.

(165) Xu, L.; Chen, W.; Mulchandani, A.; Yan, Y. Reversible Conversion of Conducting Polymer Films from Superhydrophobic to Superhydrophilic. Angew. Chem., Int. Ed. 2005, 44, 6009-6012.

(166) Strover, L.; Roux, C.; Malmström, J.; Pei, Y.; Williams, D. E.; Travas-Sejdic, J. Switchable Surfaces of Electroactive Polymer Brushes Grafted from Polythiophene ATRP-Macroinitiator. Synth. Met. 2012, 162, 381-390.

(167) Ravoo, B. J. Microcontact Chemistry: Surface Reactions in Nanoscale Confinement. J. Mater. Chem. 2009, 19, 8902-8906.

(168) Casanova, M. R.; Reis, R. L.; Martins, A.; Neves, N. M. Surface Biofunctionalization to Improve the Efficacy of Biomaterial Substrates to Be Used in Regenerative Medicine. Mater. Horiz. 2020, 7, 22582275.

(169) Lee, S.; Ozlu, B.; Eom, T.; Martin, D. C.; Shim, B. S. Electrically Conducting Polymers for Bio-Interfacing Electronics: From Neural and Cardiac Interfaces to Bone and Artificial Tissue Biomaterials. Biosens. Bioelectron. 2020, 170, 112620.

(170) Xie, J.; MacEwan, M. R.; Willerth, S. M.; Li, X.; Moran, D. W.; Sakiyama-Elbert, S. E.; Xia, Y. Conductive Core-Sheath Nanofibers and Their Potential Application in Neural Tissue Engineering. Adv. Funct. Mater. 2009, 19, 2312-2318.

(171) Hackett, A. J.; Malmström, J.; Travas-Sejdic, J. Functionalization of Conducting Polymers for Biointerface Applications. Prog. Polym. Sci. 2017, 70, 18-33.

(172) Ramanavicius, S.; Ramanavicius, A. Conducting Polymers in the Design of Biosensors and Biofuel Cells. Polymers 2021, 13, 49.

(173) Rahman, M.; Li, X.-B.; Lopa, N.; Ahn, S.; Lee, J.-J. Electrochemical DNA Hybridization Sensors Based on Conducting Polymers. Sensors 2015, 15, 3801-3829.

(174) Lee, J. Y.; Lee, J. W.; Schmidt, C. E. Neuroactive Conducting Scaffolds: Nerve Growth Factor Conjugation on Active EsterFunctionalized Polypyrrole. J. R. Soc., Interface 2009, 6, 801-810.

(175) Gomez, N.; Schmidt, C. E. Nerve Growth Factor-Immobilized Polypyrrole: Bioactive Electrically Conducting Polymer for Enhanced Neurite Extension. J. Biomed. Mater. Res., Part A 2007, 81A, 135-149. (176) Richardson, R. T.; Thompson, B.; Moulton, S.; Newbold, C.; Lum, M. G.; Cameron, A.; Wallace, G.; Kapsa, R.; Clark, G.; O’Leary, S. The Effect of Polypyrrole with Incorporated Neurotrophin-3 on the 
Promotion of Neurite Outgrowth from Auditory Neurons. Biomaterials 2007, 28, 513-523.

(177) Li, Y.; Neoh, K. G.; Cen, L.; Kang, E. T. Porous and Electrically Conductive Polypyrrole- Poly (Vinyl Alcohol) Composite and Its Applications as a Biomaterial. Langmuir 2005, 21, 10702-10709.

(178) Kim, D. H.; Richardson-Burns, S. M.; Hendricks, J. L.; Sequera, C.; Martin, D. C. Effect of Immobilized Nerve Growth Factor on Conductive Polymers: Electrical Properties and Cellular Response. Adv. Funct. Mater. 2007, 17, 79-86.

(179) Berezhetska, O.; Liberelle, B.; De Crescenzo, G.; Cicoira, F. A Simple Approach for Protein Covalent Grafting on Conducting Polymer Films. J. Mater. Chem. B 2015, 3, 5087-5094.

(180) Baumgartner, J.; Jönsson, J. I.; Jager, E. W. H. Switchable Presentation of Cytokines on Electroactive Polypyrrole Surfaces for Hematopoietic Stem and Progenitor Cells. J. Mater. Chem. B 2018, 6, $4665-4675$

(181) Widge, A. S.; Jeffries-El, M.; Cui, X.; Lagenaur, C. F.; Matsuoka, Y. Self-Assembled Monolayers of Polythiophene Conductive Polymers Improve Biocompatibility and Electrical Impedance of Neural Electrodes. Biosens. Bioelectron. 2007, 22, 1723-1732.

(182) Liu, X.; Yue, Z.; Higgins, M. J.; Wallace, G. G. Conducting Polymers with Immobilised Fibrillar Collagen for Enhanced Neural Interfacing. Biomaterials 2011, 32, 7309-7317.

(183) Lee, J. W.; Serna, F.; Nickels, J.; Schmidt, C. E. Carboxylic AcidFunctionalized Conductive Polypyrrole as a Bioactive Platform for Cell Adhesion. Biomacromolecules 2006, 7, 1692-1695.

(184) De Giglio, E.; Sabbatini, L.; Colucci, S.; Zambonin, G. Synthesis, Analytical Characterization, and Osteoblast Adhesion Properties on RGD-Grafted Polypyrrole Coatings on Titanium Substrates. J. Biomater. Sci., Polym. Ed. 2000, 11, 1073-1083.

(185) Wang, K.; Tang, R. Y.; Zhao, X. B.; Li, J. J.; Lang, Y. R.; Jiang, X. X.; Sun, H. J.; Lin, Q. X.; Wang, C. Y. Covalent Bonding of YIGSR and RGD to PEDOT/PSS/MWCNT-COOH Composite Material to Improve the Neural Interface. Nanoscale 2015, 7, 18677-18685.

(186) Bax, D. V.; Tipa, R. S.; Kondyurin, A.; Higgins, M. J.; Tsoutas, K.; Gelmi, A.; Wallace, G. G.; McKenzie, D. R.; Weiss, A. S.; Bilek, M. M. M. Cell Patterning via Linker-Free Protein Functionalization of an Organic Conducting Polymer (Polypyrrole) Electrode. Acta Biomater. 2012, 8, 2538-2548.

(187) Bousalem, S.; Mangeney, C.; Chehimi, M. M.; Basinska, T.; Miksa, B.; Slomkowski, S. Synthesis, Characterization and Potential Biomedical Applications of N-Succinimidyl Ester Functionalized, Polypyrrole-Coated Polystyrene Latex Particles. Colloid Polym. Sci. 2004, 282, 1301-1307.

(188) Svennersten, K.; Bolin, M. H.; Jager, E. W. H.; Berggren, M.; Richter-Dahlfors, A. Electrochemical Modulation of Epithelia Formation Using Conducting Polymers. Biomaterials 2009, 30, 62576264.

(189) Bousalem, S.; Benabderrahmane, S.; Sang, Y. Y. C.; Mangeney, C.; Chehimi, M. M. Covalent Immobilization of Human Serum Albumin onto Reactive Polypyrrole-Coated Polystyrene Latex Particles. J. Mater. Chem. 2005, 15, 3109-3116.

(190) De Giglio, E.; Sabbatini, L.; Zambonin, P. G. Development and Analytical Characterization of Cysteine-Grafted Polypyrrole Films Electrosynthesized on Ptand Ti-Substrates as Precursors of Bioactive Interfaces. J. Biomater. Sci., Polym. Ed. 1999, 10, 845-858.

(191) Strakosas, X.; Sessolo, M.; Hama, A.; Rivnay, J.; Stavrinidou, E.; Malliaras, G. G.; Owens, R. M. A Facile Biofunctionalisation Route for Solution Processable Conducting Polymer Devices. J. Mater. Chem. B 2014, 2, 2537-2545.

(192) Povlich, L. K.; Cho, J. C.; Leach, M. K.; Corey, J. M.; Kim, J.; Martin, D. C. Synthesis, Copolymerization and Peptide-Modification of Carboxylic Acid-Functionalized 3,4-Ethylenedioxythiophene (EDOTacid) for Neural Electrode Interfaces. Biochim. Biophys. Acta, Gen. Subj. 2013, 1830, 4288-4293.

(193) Lee, J. Y.; Bashur, C. A.; Milroy, C. A.; Forciniti, L.; Goldstein, A. S.; Schmidt, C. E. Nerve Growth Factor-Immobilized Electrically Conducting Fibrous Scaffolds for Potential Use in Neural Engineering Applications. IEEE Trans. Nanobioscience 2012, 11, 15-21.
(194) Maione, S.; Gil, A. M.; Fabregat, G.; Del Valle, L. J.; Triguero, J.; Laurent, A.; Jacquemin, D.; Estrany, F.; Jiménez, A. I.; Zanuy, D.; et al. Electroactive Polymer-Peptide Conjugates for Adhesive Biointerfaces. Biomater. Sci. 2015, 3, 1395-1405.

(195) Bhagwat, N.; Murray, R. E.; Shah, S. I.; Kiick, K. L.; Martin, D. C. Biofunctionalization of PEDOT Films with Laminin-Derived Peptides. Acta Biomater. 2016, 41, 235-246.

(196) Asplund, M.; von Holst, H.; Inganäs, O. Composite Biomolecule/PEDOT Materials for Neural Electrodes. Biointerphases 2008, 3, 83-93.

(197) Gelmi, A.; Higgins, M. J.; Wallace, G. G. Physical Surface and Electromechanical Properties of Doped Polypyrrole Biomaterials. Biomaterials 2010, 31, 1974-1983.

(198) Molino, P. J.; Yue, Z.; Zhang, B.; Tibbens, A.; Liu, X.; Kapsa, R. M. I.; Higgins, M. J.; Wallace, G. G. Influence of Biodopants on PEDOT Biomaterial Polymers: Using QCM-D to Characterize Polymer Interactions with Proteins and Living Cells. Adv. Mater. Interfaces 2014, 1, 1300122.

(199) Fahlgren, A.; Bratengeier, C.; Gelmi, A.; Semeins, C. M.; KleinNulend, J.; Jager, E. W. H.; Bakker, A. D. Biocompatibility of Polypyrrole with Human Primary Osteoblasts and the Effect of Dopants. PLoS One 2015, 10, No. e0134023.

(200) Qin, C.; Yue, Z.; Chao, Y.; Forster, R. J.; Maolmhuaidh, F.; Huang, X. F.; Beirne, S.; Wallace, G. G.; Chen, J. Bipolar Electroactive Conducting Polymers for Wireless Cell Stimulation. Appl. Mater. Today 2020, 21, 100804 .

(201) Molino, P. J.; Zhang, B. B.; Wallace, G. G.; Hanks, T. W. Surface Modification of Polypyrrole/Biopolymer Composites for Controlled Protein and Cellular Adhesion. Biofouling 2013, 29, 1155-1167.

(202) Molino, P. J.; Garcia, L.; Stewart, E. M.; Lamaze, M.; Zhang, B.; Harris, A. R.; Winberg, P.; Wallace, G. G. PEDOT Doped with Algal, Mammalian and Synthetic Dopants: Polymer Properties, Protein and Cell Interactions, and Influence of Electrical Stimulation on Neuronal Cell Differentiation. Biomater. Sci. 2018, 6, 1250-1261.

(203) Bissell, M. J.; Hall, H. G.; Parry, G. How Does the Extracellular Matrix Direct Gene Expression? J. Theor. Biol. 1982, 99, 31-68.

(204) Boudreau, N.; Bissell, M. J. Extracellular Matrix Signaling: Integration of Form and Function in Normal and Malignant Cells. Curr. Opin. Cell Biol. 1998, 10, 640-646.

(205) Wang, N.; Butler, J. P.; Ingber, D. E. Mechanotransduction across the Cell Surface and through the Cytoskeleton. Science (Washington, DC, U. S.) 1993, 260, 1124-1127.

(206) Ingber, D. E. Mechanobiology and Diseases of Mechanotransduction. Ann. Med. 2003, 35, 564-577.

(207) Fromherz, P. Three Levels of Neuroelectronic Interfacing: Silicon Chips with Ion Channels, Nerve Cells, and Brain Tissue. Ann. N. Y. Acad. Sci. 2006, 1093, 143-160.

(208) Wu, C.; Zhu, X.; Man, T.; Chung, P. S.; Teitell, M. A.; Chiou, P. Y. Lift-off Cell Lithography for Cell Patterning with Clean Background. Lab Chip 2018, 18, 3074-3078.

(209) Kandere-Grzybowska, K.; Campbell, C.; Komarova, Y.; Grzybowski, B. A.; Borisy, G. G. Molecular Dynamics Imaging in Micropatterned Living Cells. Nat. Methods 2005, 2, 739-741.

(210) Lee, S. H.; Rho, W. Y.; Park, S. J.; Kim, J.; Kwon, O. S.; Jun, B. H. Multifunctional Self-Assembled Monolayers via Microcontact Printing and Degas-Driven Flow Guided Patterning. Sci. Rep. 2018, 8, 16763.

(211) Yiannakou, C.; Simitzi, C.; Manousaki, A.; Fotakis, C.; Ranella, A.; Stratakis, E. Cell Patterning via Laser Micro/Nano Structured Silicon Surfaces. Biofabrication 2017, 9, 025024.

(212) Razal, J. M.; Kita, M.; Quigley, A. F.; Kennedy, E.; Moulton, S. E.; Kapsa, R. M. I.; Clark, G. M.; Wallace, C. C. Wet-Spun Biodegradable Fibers on Conducting Platforms: Novel Architectures for Muscle Regeneration. Adv. Funct. Mater. 2009, 19, 3381-3388.

(213) Guo, W.; Zhang, X.; Yu, X.; Wang, S.; Qiu, J.; Tang, W.; Li, L.; Liu, H.; Wang, Z. L. Self-Powered Electrical Stimulation for Enhancing Neural Differentiation of Mesenchymal Stem Cells on GraphenePoly(3,4-Ethylenedioxythiophene) Hybrid Microfibers. ACS Nano 2016, 10, 5086-5095. 
(214) Santoro, F.; van de Burgt, Y.; Keene, S. T.; Cui, B.; Salleo, A. Enhanced Cell-Chip Coupling by Rapid Femtosecond Laser Patterning of Soft PEDOT:PSS Biointerfaces. ACS Appl. Mater. Interfaces 2017, 9, 39116-39121.

(215) Greco, F.; Fujie, T.; Ricotti, L.; Taccola, S.; Mazzolai, B.; Mattoli, V. Microwrinkled Conducting Polymer Interface for Anisotropic Multicellular Alignment. ACS Appl. Mater. Interfaces 2013, 5, 573-584.

(216) Weiss, P. Experiments on Cell and Axon Orientation in Vitro: The Role of Colloidal Exudates in Tissue Organization. J. Exp. Zool. 1945, 100, 353-386.

(217) Harrison, R. G. The Cultivation of Tissues in Extraneous Media as a Method of Morpho-genetic Study. Anat. Rec. 1912, 6, 181-193.

(218) Gisbert Roca, F.; André, F. M.; Más Estellés, J.; Monleón Pradas, M.; Mir, L. M.; Martínez-Ramos, C. BDNF-Gene Transfected Schwann Cell-Assisted Axonal Extension and Sprouting on New PLAPPy Microfiber Substrates. Macromol. Biosci. 2021, 21, 2000391.

(219) Wu, Z. L.; Qi, Y. N.; Yin, X. J.; Yang, X.; Chen, C. M.; Yu, J. Y.; Yu, J. C.; Lin, Y. M.; Hui, F.; Liu, P. L.; et al. Polymer-Based Device Fabrication and Applications Using Direct Laser Writing Technology. Polymers (Basel, Switz.) 2019, 11, 553.

(220) Ravi-Kumar, S.; Lies, B.; Zhang, X.; Lyu, H.; Qin, H. Laser Ablation of Polymers: A Review. Polym. Int. 2019, 68, 1391-1401.

(221) Karuri, N. W.; Liliensiek, S.; Teixeira, A. I.; Abrams, G.; Campbell, S.; Nealey, P. F.; Murphy, C. J. Bilogical Length Scale Topography Enhances Cell-Substratum Adhesion of Human Corneal Epithelial Cells. J. Cell Sci. 2004, 117, 3153-3164.

(222) Nguyen, A. T.; Sathe, S. R.; Yim, E. K. F. From Nano to Micro: Topographical Scale and Its Impact on Cell Adhesion, Morphology and Contact Guidance. J. Phys.: Condens. Matter 2016, 28, 183001.

(223) Pas, J.; Pitsalidis, C.; Koutsouras, D. A.; Quilichini, P. P.; Santoro, F.; Cui, B.; Gallais, L.; O'Connor, R. P.; Malliaras, G. G.; Owens, R. M. Neurospheres on Patterned PEDOT:PSS Microelectrode Arrays Enhance Electrophysiology Recordings. Adv. Biosyst. 2018, 2, 1700164

(224) Ohayon, D.; Pitsalidis, C.; Pappa, A. M.; Hama, A.; Zhang, Y.; Gallais, L.; Owens, R. M. Laser Patterning of Self-Assembled Monolayers on PEDOT:PSS Films for Controlled Cell Adhesion. Adv. Mater. Interfaces 2017, 4, 1700191.

(225) Tomaskovic-Crook, E.; Zhang, P.; Ahtiainen, A.; Kaisvuo, H.; Lee, C.-Y.; Beirne, S.; Aqrawe, Z.; Svirskis, D.; Hyttinen, J.; Wallace, G. G.; et al. Human Neural Tissues from Neural Stem Cells Using Conductive Biogel and Printed Polymer Microelectrode Arrays for 3D Electrical Stimulation. Adv. Healthcare Mater. 2019, 8, 1900425.

(226) Tullii, G.; Giona, F.; Lodola, F.; Bonfadini, S.; Bossio, C.; Varo, S.; Desii, A.; Criante, L.; Sala, C.; Pasini, M.; et al. High-Aspect-Ratio Semiconducting Polymer Pillars for 3D Cell Cultures. ACS Appl. Mater. Interfaces 2019, 11, 28125-28137.

(227) Abedi, A.; Hasanzadeh, M.; Tayebi, L. Conductive Nanofibrous Chitosan/PEDOT:PSS Tissue Engineering Scaffolds. Mater. Chem. Phys. 2019, 237, 121882.

(228) Lee, J. Y.; Bashur, C. A.; Goldstein, A. S.; Schmidt, C. E. Polypyrrole-Coated Electrospun PLGA Nanofibers for Neural Tissue Applications. Biomaterials 2009, 30, 4325-4335.

(229) Amorini, F.; Zironi, I.; Marzocchi, M.; Gualandi, I.; Calienni, M.; Cramer, T.; Fraboni, B.; Castellani, G. Electrically Controlled "Sponge Effect" of PEDOT:PSS Governs Membrane Potential and Cellular Growth. ACS Appl. Mater. Interfaces 2017, 9, 6679-6689.

(230) Gomez-Carretero, S.; Libberton, B.; Svennersten, K.; Persson, K.; Jager, E.; Berggren, M.; Rhen, M.; Richter-Dahlfors, A. RedoxActive Conducting Polymers Modulate Salmonella Biofilm Formation by Controlling Availability of Electron Acceptors. npj Biofilms Microbiomes 2017, 3, 19.

(231) Saltó, C.; Saindon, E.; Bolin, M.; Kanciurzewska, A.; Fahlman, M.; Jager, E. W. H.; Tengvall, P.; Arenas, E.; Berggren, M. Control of Neural Stem Cell Adhesion and Density by an Electronic Polymer Surface Switch. Langmuir 2008, 24, 14133-14138.
(232) Lundin, V.; Herland, A.; Berggren, M.; Jager, E. W. H.; Teixeira, A. I. Control of Neural Stem Cell Survival by Electroactive Polymer Substrates. PLoS One 2011, 6, e18624.

(233) Herland, A.; Persson, K. M.; Lundin, V.; Fahlman, M.; Berggren, M.; Jager, E. W. H.; Teixeira, A. I. Electrochemical Control of Growth Factor Presentation to Steer Neural Stem Cell Differentiation. Angew. Chem., Int. Ed. 2011, 50, 12529-12533.

(234) Wan, A. M. D.; Schur, R. M.; Ober, C. K.; Fischbach, C.; Gourdon, D.; Malliaras, G. G. Electrical Control of Protein Conformation. Adv. Mater. 2012, 24, 2501-2505.

(235) Zhang, H.; Molino, P. J.; Wallace, G. G.; Higgins, M. J. Quantifying Molecular-Level Cell Adhesion on Electroactive Conducting Polymers using Electrochemical-Single Cell Force Spectroscopy. Sci. Rep. 2015, 5, 13334.

(236) Marzocchi, M.; Gualandi, I.; Calienni, M.; Zironi, I.; Scavetta, E.; Castellani, G.; Fraboni, B. Physical and Electrochemical Properties of PEDOT:PSS as a Tool for Controlling Cell Growth. ACS Appl. Mater. Interfaces 2015, 7, 17993-18003.

(237) DeFranco, J. A.; Schmidt, B. S.; Lipson, M.; Malliaras, G. G. Photolithographic Patterning of Organic Electronic Materials. Org. Electron. 2006, 7, 22-28.

(238) Liao, C.; Zhang, M.; Yao, M. Y.; Hua, T.; Li, L.; Yan, F. Adv. Mater. 2015, 27, 7493-7527.

(239) Harris, A. R.; Molino, P. J.; Kapsa, R. M. I.; Clark, G. M.; Paolini, A. G.; Wallace, G. G. Correlation of the Impedance and Effective Electrode Area of Doped PEDOT Modified Electrodes for BrainMachine Interfaces. Analyst 2015, 140, 3164-3174.

(240) Sahalianov, I.; Singh, S. K.; Tybrandt, K.; Berggren, M.; Zozoulenko, I. The Intrinsic Volumetric Capacitance of Conducting Polymers: Pseudo-Capacitors or Double-Layer Supercapacitors? RSC Adv. 2019, 9, 42498-42508.

(241) Vomero, M.; Castagnola, E.; Ciarpella, F.; Maggiolini, E.; Goshi, N.; Zucchini, E.; Carli, S.; Fadiga, L.; Kassegne, S.; Ricci, D. Highly Stable Glassy Carbon Interfaces for Long-Term Neural Stimulation and Low-Noise Recording of Brain Activity. Sci. Rep. 2017, 7, 40332.

(242) Aqrawe, Z.; Montgomery, J.; Travas-Sejdic, J.; Svirskis, D. Conducting Polymers for Neuronal Microelectrode Array Recording and Stimulation. Sens. Actuators, B 2018, 257, 753-765.

(243) Schander, A.; Teßmann, T.; Strokov, S.; Stemmann, H.; Kreiter, A. K.; Lang, W. In-Vitro Evaluation of the Long-Term Stability of PEDOT: PSS Coated Microelectrodes for Chronic Recording and Electrical Stimulation of Neurons. 8th Annual International Conference of the IEEE Engineering in Medicine and Biology Society (EMBC) 2016, 6174-6177.

(244) Khodagholy, D.; Gelinas, J. N.; Thesen, T.; Doyle, W.; Devinsky, O.; Malliaras, G. G.; Buzsáki, G. NeuroGrid: Recording Action Potentials from the Surface of the Brain. Nat. Neurosci. 2015, 18, 310-315.

(245) Xu, L.; Gutbrod, S. R.; Ma, Y.; Petrossians, A.; Liu, Y.; Webb, R. C.; Fan, J. A.; Yang, Z.; Xu, R.; Whalen III, J. J.; et al. Materials and Fractal Designs for 3D Multifunctional Integumentary Membranes with Capabilities in Cardiac Electrotherapy. Adv. Mater. 2015, 27, $1731-1737$

(246) Khodagholy, D.; Doublet, T.; Quilichini, P.; Gurfinkel, M.; Leleux, P.; Ghestem, A.; Ismailova, E.; Hervé, T.; Sanaur, S.; Bernard, C.; Malliaras, G. G. In Vivo Recordings of Brain Activity Using Organic Transistors. Nat. Commun. 2013, 4, 1575

(247) Decataldo, F.; Cramer, T.; Martelli, D.; Gualandi, I.; Korim, W. S.; Yao, S. T.; Tessarolo, M.; Murgia, M.; Scavetta, E.; Amici, R.; Fraboni, B. Stretchable Low Impedance Electrodes for Bioelectronic Recording from Small Peripheral Nerves. Sci. Rep. 2019, 9, 10598.

(248) Ferlauto, L.; D’Angelo, A. N.; Vagni, P.; Airaghi Leccardi, M. J. I.; Mor, F. M.; Cuttaz, E. A.; Heuschkel, M. O.; Stoppini, L.; Ghezzi, D. Development and Characterization of PEDOT:PSS/Alginate Soft Microelectrodes for Application in Neuroprosthetics. Front. Neurosci. 2018, 12, 648 .

(249) Abidian, M. R.; Corey, J. M.; Kipke, D. R.; Martin, D. C. Conducting-Polymer Nanotubes Improve Electrical Properties, 
Mechanical Adhesion, Neural Attachment and Neurite Outgrowth of Neural Electrodes. Small 2010, 6, 421-429.

(250) Löffler, S.; Richter-Dahlfors, A. Phase Angle Spectroscopy on Transparent Conducting Polymer Electrodes for Real-Time Measurement of Epithelial Barrier Integrity. J. Mater. Chem. B 2015, 3, 49975000.

(251) Liu, H. Y.; Pappa, A. M.; Pavia, A.; Pitsalidis, C.; Thiburce, Q.; Salleo, A.; Owens, R. M.; Daniel, S. Self-Assembly of Mammalian-Cell Membranes on Bioelectronic Devices with Functional Transmembrane Proteins. Langmuir 2020, 36, 7325-7331.

(252) Koutsouras, D. A.; Lingstedt, L. V.; Lieberth, K.; Reinholz, J.; Mailänder, V.; Blom, P. W. M.; Gkoupidenis, P. Probing the Impedance of a Biological Tissue with PEDOT:PSS-Coated Metal Electrodes: Effect of Electrode Size on Sensing Efficiency. Adv. Healthcare Mater. 2019, 8, 1901215.

(253) Pappa, A.-M.; Liu, H.-Y.; Traberg-Christensen, W.; Thiburce, Q.; Savva, A.; Pavia, A.; Salleo, A.; Daniel, S.; Owens, R. M. Optical and Electronic Ion Channel Monitoring from Native Human Membranes. ACS Nano 2020, 14, 12538.

(254) Castagnola, V.; Descamps, E.; Lecestre, A.; Dahan, L.; Remaud, J.; Nowak, L. G.; Bergaud, C. Parylene-Based Flexible Neural Probes with PEDOT Coated Surface for Brain Stimulation and Recording. Biosens. Bioelectron. 2015, 67, 450-457.

(255) Kshirsagar, P.; Dickreuter, S.; Mierzejewski, M.; Burkhardt, C. J.; Chassé, T.; Fleischer, M.; Jones, P. D. Transparent Graphene/ PEDOT:PSS Microelectrodes for Electro- and Optophysiology. Adv. Mater. Technol. 2019, 4, 1800318.

(256) Susloparova, A.; Halliez, S.; Begard, S.; Colin, M.; Buée, L.; Pecqueur, S.; Alibart, F.; Thomy, V.; Arscott, S.; Pallecchi, E.; et al. Low Impedance and Highly Transparent Microelectrode Arrays (MEA) for in Vitro Neuron Electrical Activity Probing. Sens. Actuators, B 2021, $327,128895$.

(257) Middya, S.; Curto, V. F.; Fernández-Villegas, A.; Robbins, M.; Gurke, J.; Moonen, E. J. M.; Kaminski Schierle, G. S.; Malliaras, G. G. Microelectrode Arrays for Simultaneous Electrophysiology and Advanced Optical Microscopy. Adv. Sci. 2021, 8, 2004434.

(258) Garma, L. D.; Ferrari, L. M.; Scognamiglio, P.; Greco, F.; Santoro, F. Inkjet-Printed PEDOT:PSS Multi-Electrode Arrays for Low-Cost in Vitro Electrophysiology. Lab Chip 2019, 19, 3776-3786.

(259) Sinha, S. K.; Noh, Y.; Reljin, N.; Treich, G. M.; HajebMohammadalipour, S.; Guo, Y.; Chon, K. H.; Sotzing, G. A. ScreenPrinted PEDOT:PSS Electrodes on Commercial Finished Textiles for Electrocardiography. ACS Appl. Mater. Interfaces 2017, 9, 3752437528.

(260) Middya, S.; Curto, V. F.; Fernández-Villegas, A.; Robbins, M.; Gurke, J.; Moonen, E. J. M.; Kaminski Schierle, G. S.; Malliaras, G. G. Microelectrode Arrays for Simultaneous Electrophysiology and Advanced Optical Microscopy. Adv. Sci. 2021, 8, 2004434.

(261) Guo, X.; Xu, Y.; Ogier, S.; Ng, T. N.; Caironi, M.; Perinot, A.; Li, L.; Zhao, J.; Tang, W.; Sporea, R. A.; et al. Current Status and Opportunities of Organic Thin-Film Transistor Technologies. IEEE Trans. Electron Devices 2017, 64, 1906-1921.

(262) Paterson, A. F.; Singh, S.; Fallon, K. J.; Hodsden, T.; Han, Y.; Schroeder, B. C.; Bronstein, H.; Heeney, M.; McCulloch, I.; Anthopoulos, T. D. Recent Progress in High-Mobility Organic Transistors: A Reality Check. Adv. Mater. 2018, 30, 1801079.

(263) Strakosas, X.; Bongo, M.; Owens, R. M. The Organic Electrochemical Transistor for Biological Applications. J. Appl. Polym. Sci. 2015, 132, 41735.

(264) Rivnay, J.; Inal, S.; Salleo, A.; Owens, R. M.; Berggren, M.; Malliaras, G. G. Organic Electrochemical Transistors. Nat. Rev. Mater. 2018, 3, 17086

(265) Toss, H.; Suspène, C.; Piro, B.; Yassar, A.; Crispin, X.; Kergoat, L.; Pham, M.-C.; Berggren, M. On the Mode of Operation in Electrolyte-Gated Thin Film Transistors Based on Different Substituted Polythiophenes. Org. Electron. 2014, 15, 2420-2427.

(266) Spanu, A.; Martines, L.; Bonfiglio, A. Interfacing Cells with Organic Transistors: A Review of in Vitro and in Vivo Applications. Lab Chip 2021, 21, 795-820.
(267) Duong, D. T.; Tuchman, Y.; Chakthranont, P.; Cavassin, P.; Colucci, R.; Jaramillo, T. F.; Salleo, A.; Faria, G. C. A Universal Platform for Fabricating Organic Electrochemical Devices. Adv. Electron. Mater. 2018, 4, 1800090.

(268) Spyropoulos, G. D.; Gelinas, J. N.; Khodagholy, D. Internal IonGated Organic Electrochemical Transistor: A Building Block for Integrated Bioelectronics. Sci. Adv. 2019, 5, No. eaau7378.

(269) Friedlein, J. T.; McLeod, R. R.; Rivnay, J. Device Physics of Organic Electrochemical Transistors. Org. Electron. 2018, 63, 398-414. (270) Chen, S.; Surendran, A.; Wu, X.; Lee, S. Y.; Stephen, M.; Leong, W. L. Recent Technological Advances in Fabrication and Application of Organic Electrochemical Transistors. Adv. Mater. Technol. 2020, 5, 2000523.

(271) Venkatraman, V.; Friedlein, J. T.; Giovannitti, A.; Maria, I. P.; McCulloch, I.; McLeod, R. R.; Rivnay, J. Subthreshold Operation of Organic Electrochemical Transistors for Biosignal Amplification. Adv. Sci. 2018, 5, 1800453.

(272) Khodagholy, D.; Rivnay, J.; Sessolo, M.; Gurfinkel, M.; Leleux, P.; Jimison, L. H.; Stavrinidou, E.; Herve, T.; Sanaur, S.; Owens, R. M.; Malliaras, G. G. High Transconductance Organic Electrochemical Transistors. Nat. Commun. 2013, 4, 2133.

(273) Zhang, Y.; Li, J.; Li, R.; Sbircea, D. T.; Giovannitti, A.; Xu, J.; Xu, H.; Zhou, G.; Bian, L.; McCulloch, I.; et al. Liquid-Solid Dual-Gate Organic Transistors with Tunable Threshold Voltage for Cell Sensing. ACS Appl. Mater. Interfaces 2017, 9, 38687-38694.

(274) Lingstedt, L. V.; Ghittorelli, M.; Brückner, M.; Reinholz, J.; Crăciun, N. I.; Torricelli, F.; Mailänder, V.; Gkoupidenis, P.; Blom, P. W. M. Monitoring of Cell Layer Integrity with a Current-Driven Organic Electrochemical Transistor. Adv. Healthcare Mater. 2019, 8, 1900128.

(275) Tria, S. A.; Jimison, L. H.; Hama, A.; Bongo, M.; Owens, R. M. Validation of the Organic Electrochemical Transistor for in Vitro Toxicology. Biochim. Biophys. Acta, Gen. Subj. 2013, 1830, 4381-4390.

(276) Bernards, D. A.; Malliaras, G. G.; Toombes, G. E. S.; Gruner, S. M. Gating of an Organic Transistor through a Bilayer Lipid Membrane with Ion Channels. Appl. Phys. Lett. 2006, 89, 053505.

(277) Faria, G. C.; Duong, D. T.; Salleo, A. On the Transient Response of Organic Electrochemical Transistors. Org. Electron. 2017, 45, 215-221.

(278) Decataldo, F.; Barbalinardo, M.; Tessarolo, M.; Vurro, V.; Calienni, M.; Gentili, D.; Valle, F.; Cavallini, M.; Fraboni, B. Organic Electrochemical Transistors: Smart Devices for Real-Time Monitoring of Cellular Vitality. Adv. Mater. Technol. 2019, 4, 1900207.

(279) Alfonso, J. C. L.; Schaadt, N. S.; Schönmeyer, R.; Brieu, N.; Forestier, G.; Wemmert, C.; Feuerhake, F.; Hatzikirou, H. In-Silico Insights on the Prognostic Potential of Immune Cell Infiltration Patterns in the Breast Lobular Epithelium. Sci. Rep. 2016, 6, 33322.

(280) Romele, P.; Gkoupidenis, P.; Koutsouras, D. A.; Lieberth, K.; Kovács-Vajna, Z. M.; Blom, P. W. M.; Torricelli, F. Multiscale Real Time and High Sensitivity Ion Detection with Complementary Organic Electrochemical Transistors Amplifier. Nat. Commun. 2020, 11, 3743.

(281) Curto, V. F.; Marchiori, B.; Hama, A.; Pappa, A. M.; Ferro, M. P.; Braendlein, M.; Rivnay, J.; Fiocchi, M.; Malliaras, G. G.; Ramuz, M.; Owens, R. M. Organic Transistor Platform with Integrated Microfluidics for In-Line Multi-Parametric in Vitro Cell Monitoring. Microsystems Nanoeng. 2017, 3, 17028.

(282) Marquez, A. V.; McEvoy, N.; Pakdel, A. Organic Electrochemical Transistors (OECTs) Toward Flexible and Wearable Bioelectronics. Molecules 2020, 25, 5288.

(283) Yeung, S. Y.; Gu, X.; Tsang, C. M.; Tsao, S. W.; Hsing, I. Engineering Organic Electrochemical Transistor (OECT) to Be Sensitive Cell-Based Biosensor through Tuning of Channel Area. Sens. Actuators, A 2019, 287, 185-193.

(284) White, H. S.; Kittlesen, G. P.; Wrighton, M. S. Chemical Derivatization of an Array of Three Gold Microelectrodes with Polypyrrole: Fabrication of a Molecule-Based Transistor. J. Am. Chem. Soc. 1984, 106, 5375-5377.

(285) Ramuz, M.; Margita, K.; Hama, A.; Leleux, P.; Rivnay, J.; Bazin, I.; Owens, R. M. Optimization of a Planar All-Polymer Transistor for 
Characterization of Barrier Tissue. ChemPhysChem 2015, 16, 12101216.

(286) Donahue, M. J.; Williamson, A.; Strakosas, X.; Friedlein, J. T.; McLeod, R. R.; Gleskova, H.; Malliaras, G. G. High-Performance Vertical Organic Electrochemical Transistors. Adv. Mater. 2018, 30, 1705031 .

(287) Liang, Y.; Brings, F.; Maybeck, V.; Ingebrandt, S.; Wolfrum, B.; Pich, A.; Offenhäusser, A.; Mayer, D. Tuning Channel Architecture of Interdigitated Organic Electrochemical Transistors for Recording the Action Potentials of Electrogenic Cells. Adv. Funct. Mater. 2019, 29, 1902085 .

(288) Nissa, J.; Janson, P.; Simon, D. T.; Berggren, M. Expanding the Understanding of Organic Electrochemical Transistor Function. Appl. Phys. Lett. 2021, 118, 053301.

(289) Tang, K.; Miao, W.; Guo, S. Crosslinked PEDOT:PSS Organic Electrochemical Transistors on Interdigitated Electrodes with Improved Stability. ACS Appl. Polym. Mater. 2021, 3, 1436-1444.

(290) Lee, W.; Kobayashi, S.; Nagase, M.; Jimbo, Y.; Saito, I.; Inoue, Y.; Yambe, T.; Sekino, M.; Malliaras, G. G.; Yokota, T.; et al. Nonthrombogenic, Stretchable, Active Multielectrode Array for Electroanatomical Mapping. Sci. Adv. 2018, 4, No. eaau2426.

(291) Zhang, Q.; Leonardi, F.; Casalini, S.; Temiño, I.; Mas-Torrent, M. High Performing Solution-Coated Electrolyte-Gated Organic FieldEffect Transistors for Aqueous Media Operation. Sci. Rep. 2016, 6, 39623.

(292) Macchia, E.; Picca, R. A.; Manoli, K.; Di Franco, C.; Blasi, D.; Sarcina, L.; Ditaranto, N.; Cioffi, N.; Österbacka, R.; Scamarcio, G.; et al. About the Amplification Factors in Organic Bioelectronic Sensors. Mater. Horiz. 2020, 7, 999-1013.

(293) Macchia, E.; Tiwari, A.; Manoli, K.; Holzer, B.; Ditaranto, N.; Picca, R. A.; Cioffi, N.; Di Franco, C.; Scamarcio, G.; Palazzo, G.; et al. Label-Free and Selective Single-Molecule Bioelectronic Sensing with a Millimeter-Wide Self-Assembled Monolayer of Anti-Immunoglobulins. Chem. Mater. 2019, 31, 6476-6483.

(294) Mulla, M. Y.; Tuccori, E.; Magliulo, M.; Lattanzi, G.; Palazzo, G.; Persaud, K.; Torsi, L. Capacitance-Modulated Transistor Detects Odorant Binding Protein Chiral Interactions. Nat. Commun. 2015, 6, 6010.

(295) Mulla, M. Y.; Torsi, L.; Manoli, K. Electronic Biosensors Based on EGOFETs. Methods Enzymol. 2020, 642, 403-433.

(296) Sun, C.; Wang, X.; Auwalu, M. A. A.; Cheng, S.; Hu, W. Organic Thin Film Transistors-based Biosensors. EcoMat 2021, 3, No. e12094.

(297) Gueskine, V.; Singh, A.; Vagin, M.; Crispin, X.; Zozoulenko, I. Molecular Oxygen Activation at a Conducting Polymer: Electrochemical Oxygen Reduction Reaction at PEDOT Revisited, a Theoretical Study. J. Phys. Chem. C 2020, 124, 13263-13272.

(298) Picca, R. A.; Manoli, K.; Macchia, E.; Tricase, A.; Di Franco, C.; Scamarcio, G.; Cioffi, N.; Torsi, L. A Study on the Stability of WaterGated Organic Field-Effect-Transistors Based on a Commercial p-Type Polymer. Front. Chem. 2019, 7, 667.

(299) Torricelli, F.; Adrahtas, D. Z.; Bao, Z.; Berggren, M.; Biscarini, F.; Bonfiglio, A.; Bortolotti, C. A.; Frisbie, C. D.; Macchia, E.; Malliaras, G. G.; et al. Electrolyte-Gated Transistors for Enhanced Performance Bioelectronics. Nat. Rev. Methods Prim. 2021, 1, 66.

(300) Barra, M.; Viggiano, D.; Di Capua, R.; Di Girolamo, F.; Santoro, F.; Taglialatela, M.; Cassinese, A. Cell Viability Studies and Operation in Cellular Culture Medium of N-Type Organic Field-Effect Transistors. J. Appl. Phys. 2012, 111, 034702.

(301) Kyndiah, A.; Leonardi, F.; Tarantino, C.; Cramer, T.; MillanSolsona, R.; Garreta, E.; Montserrat, N.; Mas-Torrent, M.; Gomila, G. Bioelectronic Recordings of Cardiomyocytes with Accumulation Mode Electrolyte Gated Organic Field Effect Transistors. Biosens. Bioelectron. 2020, 150, 111844.

(302) Zhang, Y.; Li, J.; Li, R.; Sbircea, D.-T.; Giovannitti, A.; Xu, J.; Xu, H.; Zhou, G.; Bian, L.; McCulloch, I.; et al. Liquid-Solid Dual-Gate Organic Transistors with Tunable Threshold Voltage for Cell Sensing. ACS Appl. Mater. Interfaces 2017, 9, 38687-38694.

(303) Desbief, S.; di Lauro, M.; Casalini, S.; Guerin, D.; Tortorella, S.; Barbalinardo, M.; Kyndiah, A.; Murgia, M.; Cramer, T.; Biscarini, F.; et al. Electrolyte-Gated Organic Synapse Transistor Interfaced with Neurons. Org. Electron. 2016, 38, 21-28.

(304) Kyndiah, A.; Leonardi, F.; Tarantino, C.; Cramer, T.; MillanSolsona, R.; Garreta, E.; Montserrat, N.; Mas-Torrent, M.; Gomila, G. Bioelectronic Recordings of Cardiomyocytes with Accumulation Mode Electrolyte Gated Organic Field Effect Transistors. Biosens. Bioelectron. 2020, 150, 111844 .

(305) Uguz, I.; Proctor, C. M.; Curto, V. F.; Pappa, A.-M.; Donahue, M. J.; Ferro, M.; Owens, R. M.; Khodagholy, D.; Inal, S.; Malliaras, G. G. A Microfluidic Ion Pump for In Vivo Drug Delivery. Adv. Mater. 2017, 29, 1701217

(306) Williamson, A.; Rivnay, J.; Kergoat, L.; Jonsson, A.; Inal, S.; Uguz, I.; Ferro, M.; Ivanov, A.; Sjöström, T. A.; Simon, D. T.; et al. Controlling Epileptiform Activity with Organic Electronic Ion Pumps. Adv. Mater. 2015, 27, 3138-3144.

(307) Cherian, D.; Armgarth, A.; Beni, V.; Linderhed, U.; Tybrandt, K.; Nilsson, D.; Simon, D. T.; Berggren, M. Large-Area Printed Organic Electronic Ion Pumps. Flex. Print. Electron. 2019, 4, 022001.

(308) Jonsson, A.; Inal, S.; Uguz, L.; Williamson, A. J.; Kergoat, L.; Rivnay, J.; Khodagholy, D.; Berggren, M.; Bernard, C.; Malliaras, G. G.; et al. Bioelectronic Neural Pixel: Chemical Stimulation and Electrical Sensing at the Same Site. Proc. Natl. Acad. Sci. U. S. A. 2016, 113, 94409445.

(309) Bernacka-Wojcik, I.; Huerta, M.; Tybrandt, K.; Karady, M.; Mulla, M. Y.; Poxson, D. J.; Gabrielsson, E. O.; Ljung, K.; Simon, D. T.; Berggren, M.; et al. Implantable Organic Electronic Ion Pump Enables ABA Hormone Delivery for Control of Stomata in an Intact Tobacco Plant. Small 2019, 15, 1902189.

(310) Tybrandt, K.; Larsson, K. C.; Kurup, S.; Simon, D. T.; Kjäll, P.; Isaksson, J.; Sandberg, M.; Jager, E. W. H.; Richter-Dahlfors, A.; Berggren, M. Translating Electronic Currents to Precise AcetylcholineInduced Neuronal Signaling Using an Organic Electrophoretic Delivery Device. Adv. Mater. 2009, 21, 4442-4446.

(311) Jonsson, A.; Sjöström, T. A.; Tybrandt, K.; Berggren, M.; Simon, D. T. Chemical Delivery Array with Millisecond Neurotransmitter Release. Sci. Adv. 2016, 2, 1601340.

(312) Jakešová, M.; Sjöström, T. A.; Đerek, V.; Poxson, D.; Berggren, M.; Głowacki, E. D.; Simon, D. T. Wireless Organic Electronic Ion Pumps Driven by Photovoltaics. npj Flex. Electron. 2019, 3, 14.

(313) Isaksson, J.; Nilsson, D.; Kjäll, P.; Robinson, N. D.; RichterDahlfors, A.; Berggren, M. Electronically Controlled PH Gradients and Proton Oscillations. Org. Electron. 2008, 9, 303-309.

(314) Simon, D. T.; Kurup, S.; Larsson, K. C.; Hori, R.; Tybrandt, K.; Goiny, M.; Jager, E. W. H.; Berggren, M.; Canlon, B.; Richter-Dahlfors, A. Organic Electronics for Precise Delivery of Neurotransmitters to Modulate Mammalian Sensory Function. Nat. Mater. 2009, 8, 742.

(315) Isaksson, J.; Kjäll, P.; Nilsson, D.; Robinson, N.; Berggren, M.; Richter-Dahlfors, A. Electronic Control of Ca2+ Signalling in Neuronal Cells Using an Organic Electronic Ion Pump. Nat. Mater. 2007, 6, 673679.

(316) Tybrandt, K.; Gabrielsson, E. O.; Berggren, M. Toward Complementary Ionic Circuits: The Npn Ion Bipolar Junction Transistor. J. Am. Chem. Soc. 2011, 133, 10141-10145.

(317) Tortiglione, C.; Antognazza, M. R.; Tino, A.; Bossio, C.; Marchesano, V.; Bauduin, A.; Zangoli, M.; Morata, S. V.; Lanzani, G. Semiconducting Polymers Are Light Nanotransducers in Eyeless Animals. Sci. Adv. 2017, 3, No. e1601699.

(318) Ghezzi, D.; Antognazza, M. R.; MacCarone, R.; Bellani, S.; Lanzarini, E.; Martino, N.; Mete, M.; Pertile, G.; Bisti, S.; Lanzani, G.; et al. A Polymer Optoelectronic Interface Restores Light Sensitivity in Blind Rat Retinas. Nat. Photonics 2013, 7, 400-406.

(319) Chen, R.; Gore, F.; Nguyen, Q.-A.; Ramakrishnan, C.; Patel, S.; Kim, S. H.; Raffiee, M.; Kim, Y. S.; Hsueh, B.; Krook-Magnusson, E.; et al. Deep Brain Optogenetics without Intracranial Surgery. Nat. Biotechnol. 2021, 39, 161-164.

(320) Silverå-Ejneby, M.; Jakešová, M.; Ferrero, J. J.; Migliaccio, L.; Zhao, Z.; Berggren, M.; Khodagholy, D.; Đerek, V.; Gelinas, J.; Głowacki, E. D. A Chronic Photocapacitor Implant for Noninvasive 
Neurostimulation with Deep Red Light. bioRxiv 2020, DOI: 10.1101/ 2020.07.01.182113.

(321) Martino, N.; Feyen, P.; Porro, M.; Bossio, C.; Zucchetti, E.; Ghezzi, D.; Benfenati, F.; Lanzani, G.; Antognazza, M. R. Photothermal Cellular Stimulation in Functional Bio-Polymer Interfaces. Sci. Rep. 2015, 5, 8911 .

(322) Rand, D.; Jakešová, M.; Lubin, G.; Vẻbraitè, I.; David-Pur, M.; Đerek, V.; Cramer, T.; Sariciftci, N. S.; Hanein, Y.; Głowacki, E. D. Direct Electrical Neurostimulation with Organic Pigment Photocapacitors. Adv. Mater. 2018, 30, 1707292.

(323) Silverå Ejneby, M.; Migliaccio, L.; Gicevičius, M.; Đerek, V.; Jakešová, M.; Elinder, F.; Głowacki, E. D. Extracellular Photovoltage Clamp Using Conducting Polymer-Modified Organic Photocapacitors. Adv. Mater. Technol. 2020, 5, 1900860.

(324) Lodola, F.; Rosti, V.; Tullii, G.; Desii, A.; Tapella, L.; Catarsi, P.; Lim, D.; Moccia, F.; Antognazza, M. R. Conjugated Polymers Optically Regulate the Fate of Endothelial Colony-Forming Cells. Sci. Adv. 2019, 5 , No. eaav4620.

(325) Đerek, V.; Rand, D.; Migliaccio, L.; Hanein, Y.; Głowacki, E. D. Untangling Photofaradaic and Photocapacitive Effects in Organic Optoelectronic Stimulation Devices. Front. Bioeng. Biotechnol. 2020, 8, 284.

(326) Hopkins, J.; Travaglini, L.; Lauto, A.; Cramer, T.; Fraboni, B.; Seidel, J.; Mawad, D. Photoactive Organic Substrates for Cell Stimulation: Progress and Perspectives. Adv. Mater. Technol. 2019, 4, 1800744.

(327) Vaquero, S.; Bossio, C.; Bellani, S.; Martino, N.; Zucchetti, E.; Lanzani, G.; Antognazza, M. R. Conjugated Polymers for the Optical Control of the Electrical Activity of Living Cells. J. Mater. Chem. B 2016, 4, 5272-5283.

(328) Srivastava, S. B.; Melikov, R.; Yildiz, E.; Dikbas, U. M.; Sadeghi, S.; Kavakli, I. H.; Sahin, A.; Nizamoglu, S. Bulk-Heterojunction Photocapacitors with High Open-Circuit Voltage for Low Light Intensity Photostimulation of Neurons. J. Mater. Chem. C 2021, 9, 1755

(329) Jakesova, M.; Silvera Ejneby, M.; Đerek, V.; Schmidt, T.; Gryszel, M.; Brask, J.; Schindl, R.; Simon, D. T.; Berggren, M.; Elinder, F.; Glowacki, E. D. Optoelectronic Control of Single Cells Using Organic Photocapacitors. Sci. Adv. 2019, 5, No. eaav5265.

(330) Benfenati, V.; Martino, N.; Antognazza, M. R.; Pistone, A.; Toffanin, S.; Ferroni, S.; Lanzani, G.; Muccini, M. Organic Polymers: Photostimulation of Whole-Cell Conductance in Primary Rat Neocortical Astrocytes Mediated by Organic Semiconducting Thin Films (Adv. Healthcare Mater. 3/2014). Adv. Healthcare Mater. 2014, 3, 306. (331) Zangoli, M.; Di Maria, F.; Zucchetti, E.; Bossio, C.; Antognazza, M. R.; Lanzani, G.; Mazzaro, R.; Corticelli, F.; Baroncini, M.; Barbarella, G. Engineering Thiophene-Based Nanoparticles to Induce Phototransduction in Live Cells under Illumination. Nanoscale 2017, 9, 9202-9209.

(332) Abdel Aziz, I.; Malferrari, M.; Roggiani, F.; Tullii, G.; Rapino, S.; Antognazza, M. R. Light-Triggered Electron Transfer between a Conjugated Polymer and Cytochrome $\mathrm{C}$ for Optical Modulation of Redox Signaling. iScience 2020, 23, 101091.

(333) Antognazza, M. R.; Abdel Aziz, I.; Lodola, F. Use of Exogenous and Endogenous Photomediators as Efficient ROS Modulation Tools: Results and Perspectives for Therapeutic Purposes. Oxid. Med. Cell. Longevity 2019, 2019, 2867516.

(334) Moros, M.; Lewinska, A.; Onorato, G.; Antognazza, M. R.; Di Francesca, M.; Blasio, M.; Lanzani, G.; Tino, A.; Wnuk, M.; Tortiglione, C. Light-Triggered Modulation of Cell Antioxidant Defense by Polymer Semiconducting Nanoparticles in a Model Organism. MRS Commun. 2018, 8, 918-925.

(335) Gerasimenko, T.; Nikulin, S.; Zakharova, G.; Poloznikov, A.; Petrov, V.; Baranova, A.; Tonevitsky, A. Impedance Spectroscopy as a Tool for Monitoring Performance in 3D Models of Epithelial Tissues. Front. Bioeng. Biotechnol. 2020, 7, 474.

(336) Tu, D.; Kergoat, L.; Crispin, X.; Berggren, M.; Forchheimer, R. Transient Analysis of Electrolyte-Gated Organic Field-Effect Transistors. Proc. SPIE 2012, 84780L.
(337) Kaphle, V.; Paudel, P. R.; Dahal, D.; Radha Krishnan, R. K.; Lüssem, B. Finding the Equilibrium of Organic Electrochemical Transistors. Nat. Commun. 2020, 11, 2515.

(338) Melzer, K.; Brändlein, M.; Popescu, B.; Popescu, D.; Lugli, P.; Scarpa, G. Characterization and Simulation of Electrolyte-Gated Organic Field-Effect Transistors. Faraday Discuss. 2014, 174, 399-411.

(339) Bernards, D. A.; Malliaras, G. G. Steady-State and Transient Behavior of Organic Electrochemical Transistors. Adv. Funct. Mater. 2007, 17, 3538-3544.

(340) Colucci, R.; Barbosa, H. F. D. P.; Günther, F.; Cavassin, P.; Faria, G. C. Recent Advances in Modeling Organic Electrochemical Transistors. Flexible and Printed Electronics 2020, 5, 013001.

(341) Szymanski, M. Z.; Tu, D.; Forchheimer, R. 2-D Drift-Diffusion Simulation of Organic Electrochemical Transistors. IEEE Trans. Electron Devices 2017, 64, 5114-5120.

(342) Paudel, P. R.; Kaphle, V.; Dahal, D.; Radha Krishnan, R. K.; Lüssem, B. Tuning the Transconductance of Organic Electrochemical Transistors. Adv. Funct. Mater. 2021, 31, 2004939.

(343) Nissa, J.; Janson, P.; Berggren, M.; Simon, D. T. The Role of Relative Capacitances in Impedance Sensing with Organic Electrochemical Transistors. Adv. Electron. Mater. 2021, 7, 2001173.

(344) Bernards, D. A.; MacAya, D. J.; Nikolou, M.; Defranco, J. A.; Takamatsu, S.; Malliaras, G. G. Enzymatic Sensing with Organic Electrochemical Transistors. J. Mater. Chem. 2008, 18, 116-120.

(345) Srinivasan, B.; Kolli, A. R.; Esch, M. B.; Abaci, H. E.; Shuler, M. L.; Hickman, J. J. TEER Measurement Techniques for In Vitro Barrier Model Systems. J. Lab. Autom. 2015, 20, 107-126.

(346) Bard, A. J.; Faulkner, L. R. Electrochem. Methods: Fundamentals and Applications 2001, 2, 580-632.

(347) Barsoukov, E.; Macdonald, J. R. Impendance Spectroscopy; John Wiley \& Sons, Inc., 2018

(348) Fariat, G. C.; Duongt, D. T.; Salleo, A.; Polyzoidis, C. A.; Logothetidis, S.; Rivnay, J.; Owens, R.; Malliaras, G. G. Organic Electrochemical Transistors as Impedance Biosensors. MRS Commun. 2014, 4, 189-194.

(349) Benson, K.; Cramer, S.; Galla, H. J. Impedance-Based Cell Monitoring: Barrier Properties and Beyond. Fluids Barriers CNS 2013 $10,5$.

(350) Ramuz, M.; Hama, A.; Rivnay, J.; Leleux, P.; Owens, R. M. Monitoring of Cell Layer Coverage and Differentiation with the Organic Electrochemical Transistor. J. Mater. Chem. B 2015, 3, 59715977.

(351) Hassan, Q.; Ahmadi, S.; Kerman, K. Recent Advances in Monitoring Cell Behavior Using Cell-Based Impedance Spectroscopy. Micromachines 2020, 11, 590.

(352) Rivnay, J.; Ramuz, M.; Leleux, P.; Hama, A.; Huerta, M.; Owens, R. M. Organic Electrochemical Transistors for Cell-Based Impedance Sensing. Appl. Phys. Lett. 2015, 106, 043301.

(353) Yang, N. J.; Hinner, M. J. Getting across the Cell Membrane: An Overview for Small Molecules, Peptides, and Proteins. Methods Mol. Biol. 2015, 1266, 29-53.

(354) Singer, S.; Nicolson, G. L. The Fluid Mosaic Model of the Structure of Cell Membranes. Science (Washington, DC, U. S.) 1972, $175,720-731$.

(355) Goñi, F. M. The Basic Structure and Dynamics of Cell Membranes: An Update of the Singer-Nicolson Model. Biochim. Biophys. Acta, Biomembr. 2014, 1838, 1467-1476.

(356) Bernardino de la Serna, J.; Schütz, G. J.; Eggeling, C.; Cebecauer, M. There Is No Simple Model of the Plasma Membrane Organization. Front. Cell Dev. Biol. 2016, 4, 106.

(357) Knobloch, J.; Suhendro, D. K.; Zieleniecki, J. L.; Shapter, J. G.; Köper, I. Membrane-Drug Interactions Studied Using Model Membrane Systems. Saudi J. Biol. Sci. 2015, 22, 714-718.

(358) Sarkis, J.; Vié, V. Biomimetic Models to Investigate Membrane Biophysics Affecting Lipid-Protein Interaction. Front. Bioeng. Biotechnol. 2020, 8, 270.

(359) Stein, H.; Spindler, S.; Bonakdar, N.; Wang, C.; Sandoghdar, V. Production of Isolated Giant Unilamellar Vesicles under High Salt Concentrations. Front. Physiol. 2017, 8, 63. 
(360) Langmuir, I. The Constitution and Fundamental Properties of Solids and Liquids. Part I. Solids. J. Am. Chem. Soc. 1916, 38, 22212295.

(361) Cavassin, P.; Pappa, A.-M.; Pitsalidis, C.; F. P. Barbosa, H.; Colucci, R.; Saez, J.; Tuchman, Y.; Salleo, A.; Faria, G. C.; Owens, R. M. Organic Transistors Incorporating Lipid Monolayers for Drug Interaction Studies. Adv. Mater. Technol. 2020, 5, 1900680.

(362) Pitsalidis, C.; Pappa, A. M.; Porel, M.; Artim, C. M.; Faria, G. C.; Duong, D. D.; Alabi, C. A.; Daniel, S.; Salleo, A.; Owens, R. M. Biomimetic Electronic Devices for Measuring Bacterial Membrane Disruption. Adv. Mater. 2018, 30, 1803130.

(363) Mueller, P.; Rudin, D. O.; Ti Tien, H.; Wescott, W. C. Reconstitution of Cell Membrane Structure in Vitro and Its Transformation into an Excitable System. Nature 1962, 194, 979-980.

(364) Boheim, G. Statistical Analysis of Alamethicin Channels in Black Lipid Membranes. J. Membr. Biol. 1974, 19, 277-303.

(365) Tamm, L. K.; McConnell, H. M. Supported Phospholipid Bilayers. Biophys. J. 1985, 47, 105-113.

(366) Chadli, M.; Rebaud, S.; Maniti, O.; Tillier, B.; Cortès, S.; GirardEgrot, A. New Tethered Phospholipid Bilayers Integrating Functional G-Protein-Coupled Receptor Membrane Proteins. Langmuir 2017, 33, 10385-10401.

(367) Knoll, W.; Frank, C. W.; Heibel, C.; Naumann, R.; Offenhäusser, A.; Rühe, J.; Schmidt, E. K.; Shen, W. W.; Sinner, A. Functional Tethered Lipid Bilayers. Rev. Mol. Biotechnol. 2000, 74, 137-158.

(368) Santoro, F.; Lubrano, C.; Matrone, G. M.; Iaconis, G. New Frontiers for Selective Biosensing with Biomembrane-Based Organic Transistors. ACS Nano 2020, 14, 12271-12280.

(369) Angione, M. D.; Cotrone, S.; Magliulo, M.; Mallardi, A.; Altamura, D.; Giannini, C.; Cioffi, N.; Sabbatini, L.; Fratini, E.; Baglioni, P.; et al. Interfacial Electronic Effects in Functional Biolayers Integrated into Organic Field-Effect Transistors. Proc. Natl. Acad. Sci. U. S. A. 2012, 109, 6429-6434.

(370) Magliulo, M.; Mallardi, A.; Mulla, M. Y.; Cotrone, S.; Pistillo, B. R.; Favia, P.; Vikholm-Lundin, I.; Palazzo, G.; Torsi, L. ElectrolyteGated Organic Field-Effect Transistor Sensors Based on Supported Biotinylated Phospholipid Bilayer. Adv. Mater. 2013, 25, 2090-2094.

(371) Zhang, Y.; Inal, S.; Hsia, C. Y.; Ferro, M.; Ferro, M.; Daniel, S.; Owens, R. M. Supported Lipid Bilayer Assembly on PEDOT:PSS Films and Transistors. Adv. Funct. Mater. 2016, 26, 7304-7313.

(372) Morigaki, K.; Tawa, K. Vesicle Fusion Studied by Surface Plasmon Resonance and Surface Plasmon Fluorescence Spectroscopy. Biophys. J. 2006, 91, 1380-1387.

(373) Zhang, Y.; Wustoni, S.; Savva, A.; Giovannitti, A.; McCulloch, I.; Inal, S. Lipid Bilayer Formation on Organic Electronic Materials. J. Mater. Chem. C 2018, 6, 5218-5227.

(374) Hohner, A. O.; David, M. P. C.; Rädler, J. O. Controlled Solvent-Exchange Deposition of Phospholipid Membranes onto Solid Surfaces. Biointerphases 2010, 5, 1-8.

(375) Jackman, J. A.; Cho, N. J. Supported Lipid Bilayer Formation: Beyond Vesicle Fusion. Langmuir 2020, 36, 1387-1400.

(376) Su, H.; Liu, H. Y.; Pappa, A. M.; Hidalgo, T. C.; Cavassin, P.; Inal, S.; Owens, R. M.; Daniel, S. Facile Generation of BiomimeticSupported Lipid Bilayers on Conducting Polymer Surfaces for Membrane Biosensing. ACS Appl. Mater. Interfaces 2019, 11, 4379943810 .

(377) Liu, H. Y.; Pappa, A. M.; Hidalgo, T. C.; Inal, S.; Owens, R. M.; Daniel, S. Biomembrane-Based Organic Electronic Devices for LigandReceptor Binding Studies. Anal. Bioanal. Chem. 2020, 412, 6265-6273. (378) Kawan, M.; Hidalgo, T. C.; Du, W.; Pappa, A.-M.; Owens, R. M.; McCulloch, I.; Inal, S. Monitoring Supported Lipid Bilayers with NType Organic Electrochemical Transistors. Mater. Horiz. 2020, 7, 2348-2358.

(379) Pappa, A.-M.; Liu, H.-Y.; Traberg-Christensen, W.; Thiburce, Q.; Savva, A.; Pavia, A.; Salleo, A.; Daniel, S.; Owens, R. M. Optical and Electronic Ion Channel Monitoring from Native Human Membranes. ACS Nano 2020, 14, 12538.
(380) Tang, T.; Savva, A.; Traberg, W. C.; Xu, C.; Thiburce, Q.; Liu, H.-Y.; Pappa, A.-M.; Martinelli, E.; Withers, A.; Cornelius, M. Functional Infectious Nanoparticle Detector: Finding Viruses by Detecting Their Host Entry Functions Using Organic Bioelectronic Devices. ACS Nano 2021, 15, 18142.

(381) Bint E Naser, S. F.; Su, H.; Liu, H.-Y.; Manzer, Z. A.; Chao, Z.; Roy, A.; Pappa, A.-M.; Salleo, A.; Owens, R. M.; Daniel, S. Detection of Ganglioside-Specific Toxin Binding with Biomembrane-Based Bioelectronic Sensors. ACS Appl. Bio Mater. 2021, 4, 7942.

(382) Feher, J. Active Transport: Pumps and Exchangers. In Quantitative Human Physiology; Feher, J., Ed.; Academic Press, 2012; pp 134-140.

(383) Feher, J. The Origin of the Resting Membrane Potential. In Quantitative Human Physiology; Feher, J., Ed.; Academic Press, 2012; pp 205-214.

(384) Feher, J. The Action Potential. In Quantitative Human Physiology; Feher, J., Ed.; Academic Press, 2012; Vol. 24, pp 215-226. (385) Feher, J. The Cardiac Action Potential. In Quantitative Human Physiology; Feher, J., Ed.; Academic Press, 2012; pp 458-466.

(386) Feher, J. Propagation of the Action Potential. In Quantitative Human Physiology; Feher, J., Ed.; Academic Press, 2012; pp 227-235.

(387) Herreras, O. Local Field Potentials: Myths and Misunderstandings. Front. Neural Circuits 2016, 10, 101.

(388) Fang, Y.; Li, X.; Fang, Y. Organic Bioelectronics for Neural Interfaces. J. Mater. Chem. C 2015, 3, 6424-6430.

(389) Burnstine-Townley, A.; Eshel, Y.; Amdursky, N. Conductive Scaffolds for Cardiac and Neuronal Tissue Engineering: Governing Factors and Mechanisms. Adv. Funct. Mater. 2020, 30, 1901369.

(390) Gu, X.; Yao, C.; Liu, Y.; Hsing, I. M. 16-Channel Organic Electrochemical Transistor Array for In Vitro Conduction Mapping of Cardiac Action Potential. Adv. Healthcare Mater. 2016, 5, 2345-2351.

(391) Gu, X.; Yeung, S. Y.; Chadda, A.; Poon, E. N. Y.; Boheler, K. R.; Hsing, I. M. Organic Electrochemical Transistor Arrays for In Vitro Electrophysiology Monitoring of 2D and 3D Cardiac Tissues. Adv. Biosyst. 2019, 3, 1800248.

(392) Liang, Y.; Ernst, M.; Brings, F.; Kireev, D.; Maybeck, V.; Offenhäusser, A.; Mayer, D. High Performance Flexible Organic Electrochemical Transistors for Monitoring Cardiac Action Potential. Adv. Healthcare Mater. 2018, 7, 1800304.

(393) Zhao, Y.; Inayat, S.; Dikin, D. A.; Singer, J. H.; Ruoff, R. S.; Troy, J. B. Patch Clamp Technique: Review of the Current State of the Art and Potential Contributions from Nanoengineering. Proc. Inst. Mech. Eng., Part N 2008, 222, 1-11.

(394) Grienberger, C.; Konnerth, A. Imaging Calcium in Neurons. Neuron 2012, 73, 862-885.

(395) Cui, X.; Lee, V. A.; Raphael, Y.; Wiler, J. A.; Hetke, J. F.; Anderson, D. J.; Martin, D. C. Surface Modification of Neural Recording Electrodes with Conducting Polymer/Biomolecule Blends. J. Biomed. Mater. Res. 2001, 56, 261-272.

(396) Collazos-Castro, J. E.; Hernández-Labrado, G. R.; Polo, J. L.; García-Rama, C. N-Cadherin- and L1-Functionalised Conducting Polymers for Synergistic Stimulation and Guidance of Neural Cell Growth. Biomaterials 2013, 34, 3603-3617.

(397) Cuttaz, E.; Goding, J.; Vallejo-Giraldo, C.; Aregueta-Robles, U.; Lovell, N.; Ghezzi, D.; Green, R. A. Conductive Elastomer Composites for Fully Polymeric, Flexible Bioelectronics. Biomater. Sci. 2019, 7, $1372-1385$.

(398) Feng, Z. Q.; Wu, J.; Cho, W.; Leach, M. K.; Franz, E. W.; Naim, Y. I.; Gu, Z. Z.; Corey, J. M.; Martin, D. C. Highly Aligned Poly(3,4Ethylene Dioxythiophene) (PEDOT) Nano- and Microscale Fibers and Tubes. Polymer 2013, 54, 702-708.

(399) Aggas, J. R.; Abasi, S.; Phipps, J. F.; Podstawczyk, D. A.; Guiseppi-Elie, A. Microfabricated and 3-D Printed Electroconductive Hydrogels of PEDOT:PSS and Their Application in Bioelectronics. Biosens. Bioelectron. 2020, 168, 112568.

(400) Dominguez-Alfaro, A.; Alegret, N.; Arnaiz, B.; Salsamendi, M.; Mecerreyes, D.; Prato, M. Toward Spontaneous Neuronal Differentiation of SH-SY5Y Cells Using Novel Three-Dimensional Electro- 
polymerized Conductive Scaffolds. ACS Appl. Mater. Interfaces 2020, 12, 57330-57342.

(401) Krukiewicz, K.; Chudy, M.; Vallejo-Giraldo, C.; Skorupa, M.; Więclawska, D.; Turczyn, R.; Biggs, M. Fractal Form PEDOT/Au Assemblies as Thin-Film Neural Interface Materials. Biomed. Mater. 2018, 13, 054102 .

(402) Krukiewicz, K.; Chudy, M.; Gregg, S.; Biggs, M. J. P. The Synergistic Effects of Gold Particles and Dexamethasone on the Electrochemical and Biological Performance of PEDOT Neural Interfaces. Polymers (Basel, Switz.) 2019, 11, 67.

(403) Lee, S.; Eom, T.; Kim, M. K.; Yang, S. G.; Shim, B. S. Durable Soft Neural Micro-Electrode Coating by an Electrochemical Synthesis of PEDOT:PSS/Graphene Oxide Composites. Electrochim. Acta 2019, 313, 79-90.

(404) Lichtenstein, M. P.; Carretero, N. M.; Pérez, E.; Pulido-Salgado, M.; Moral-Vico, J.; Solà, C.; Casañ-Pastor, N.; Suñol, C. Biosafety Assessment of Conducting Nanostructured Materials by Using CoCultures of Neurons and Astrocytes. NeuroToxicology 2018, 68, 115125.

(405) Luo, X.; Weaver, C. L.; Zhou, D. D.; Greenberg, R.; Cui, X. T. Highly Stable Carbon Nanotube Doped Poly(3,4-Ethylenedioxythiophene) for Chronic Neural Stimulation. Biomaterials 2011, 32, 55515557.

(406) Luo, X.; Weaver, C. L.; Tan, S.; Cui, X. T. Pure Graphene Oxide Doped Conducting Polymer Nanocomposite for Bio-Interfacing. J. Mater. Chem. B 2013, 1, 1340-1348.

(407) Mandelli, J. S.; Koepp, J.; Hama, A.; Sanaur, S.; Rae, G. A.; Rambo, C. R. Cell Viability and Cytotoxicity of Inkjet-Printed Flexible Organic Electrodes on Parylene C. Biomed. Microdevices 2021, 23, 2.

(408) Nyberg, T.; Inganäs, O.; Jerregård, H. Polymer Hydrogel Microelectrodes for Neural Communication. Biomed. Microdevices 2002, 4, 43-52.

(409) Tadayyon, G.; Krukiewicz, K.; Britton, J.; Larrañaga, A.; VallejoGiraldo, C.; Fernandez-Yague, M.; Guo, Y.; Orpella-Aceret, G.; Li, L.; Poudel, A.; et al. In Vitro Analysis of a Physiological Strain Sensor Formulated from a PEDOT:PSS Functionalized Carbon NanotubePoly(Glycerol Sebacate Urethane) Composite. Mater. Sci. Eng., C 2021, 121, 111857.

(410) Vallejo-Giraldo, C.; Krukiewicz, K.; Calaresu, I.; Zhu, J.; Palma, M.; Fernandez-Yague, M.; McDowell, B. W.; Peixoto, N.; Farid, N.; O'Connor, G.; et al. Attenuated Glial Reactivity on Topographically Functionalized Poly(3,4-Ethylenedioxythiophene):P-Toluene Sulfonate (PEDOT:PTS) Neuroelectrodes Fabricated by Microimprint Lithography. Small 2018, 14, 1800863.

(411) Wu, B.; Cao, B.; Taylor, I. M.; Woeppel, K.; Cui, X. T. Facile Synthesis of a 3,4-Ethylene-Dioxythiophene (EDOT) Derivative for Ease of Bio-Functionalization of the Conducting Polymer PEDOT. Front. Chem. 2019, 7, 178.

(412) Yang, J.; Kim, D. H.; Hendricks, J. L.; Leach, M.; Northey, R.; Martin, D. C. Ordered Surfactant-Templated Poly(3,4-Ethylenedioxythiophene) (PEDOT) Conducting Polymer on Microfabricated Neural Probes. Acta Biomater. 2005, 1, 125-136.

(413) Magaz, A.; Spencer, B. F.; Hardy, J. G.; Li, X.; Gough, J. E.; Blaker, J. J. Modulation of Neuronal Cell Affinity on PEDOT-PSS Nonwoven Silk Scaffolds for Neural Tissue Engineering. ACS Biomater. Sci. Eng. 2020, 6, 6906-6916.

(414) Mantione, D.; del Agua, I.; Schaafsma, W.; Diez-Garcia, J.; Castro, B.; Sardon, H.; Mecerreyes, D. Poly(3,4-Ethylenedioxythiophene):GlycosAminoGlycan Aqueous Dispersions: Toward Electrically Conductive Bioactive Materials for Neural Interfaces. Macromol. Biosci. 2016, 16, 1227-1238.

(415) Krukiewicz, K.; Kowalik, A.; Czerwinska-Glowka, D.; Biggs, M. J. P. Electrodeposited Poly(3,4-Ethylenedioxypyrrole) Films as Neural Interfaces: Cytocompatibility and Electrochemical Studies. Electrochim. Acta 2019, 302, 21-30.

(416) Moral-Vico, J.; Carretero, N. M.; Pérez, E.; Suñol, C.; Lichtenstein, M.; Casañ-Pastor, N. Dynamic Electrodeposition of Aminoacid-Polypyrrole on Aminoacid-PEDOT Substrates: Conduct- ing Polymer Bilayers as Electrodes in Neural Systems. Electrochim. Acta 2013, 111, 250-260.

(417) Teshima, T.; Nakashima, H.; Kasai, N.; Sasaki, S.; Tanaka, A.; Tsukada, S.; Sumitomo, K. Mobile Silk Fibroin Electrode for Manipulation and Electrical Stimulation of Adherent Cells. Adv. Funct. Mater. 2016, 26, 8185-8193.

(418) Green, R. A.; Lovell, N. H.; Poole-Warren, L. A. Impact of CoIncorporating Laminin Peptide Dopants and Neurotrophic Growth Factors on Conducting Polymer Properties. Acta Biomater. 2010, 6, $63-71$.

(419) Baek, S.; Green, R. A.; Poole-Warren, L. A. Effects of Dopants on the Biomechanical Properties of Conducting Polymer Films on Platinum Electrodes. J. Biomed. Mater. Res., Part A 2014, 102, 27432754.

(420) Cellot, G.; Lagonegro, P.; Tarabella, G.; Scaini, D.; Fabbri, F.; Iannotta, S.; Prato, M.; Salviati, G.; Ballerini, L. PEDOT:PSS Interfaces Support the Development of Neuronal Synaptic Networks with Reduced Neuroglia Response in Vitro. Front. Neurosci. 2016, 9, 521.

(421) Charkhkar, H.; Frewin, C.; Nezafati, M.; Knaack, G. L.; Peixoto, N.; Saddow, S. E.; Pancrazio, J. J. Use of Cortical Neuronal Networks for in Vitro Material Biocompatibility Testing. Biosens. Bioelectron. 2014, 53, 316-323.

(422) Che, J.; Xiao, Y.; Zhu, X.; Sun, X. Electro-Synthesized PEDOT/ Glutamate Chemically Modified Electrode: A Combination of Electrical and Biocompatible Features. Polym. Int. 2008, 57, 750-755.

(423) Song, H. K.; Toste, B.; Ahmann, K.; Hoffman-Kim, D.; Palmore, G. T. R. Micropatterns of Positive Guidance Cues Anchored to Polypyrrole Doped with Polyglutamic Acid: A New Platform for Characterizing Neurite Extension in Complex Environments. Biomaterials 2006, 27, 473-484.

(424) Stewart, E. M.; Wu, Z.; Huang, X. F.; Kapsa, R. M. I.; Wallace, G. G. Use of Conducting Polymers to Facilitate Neurite Branching in Schizophrenia-Related Neuronal Development. Biomater. Sci. 2016, 4, 1244-1251.

(425) Wadhwa, R.; Lagenaur, C. F.; Cui, X. T. Electrochemically Controlled Release of Dexamethasone from Conducting Polymer Polypyrrole Coated Electrode. J. Controlled Release 2006, 110, 531541.

(426) Manzari-Tavakoli, A.; Tarasi, R.; Sedghi, R.; Moghimi, A.; Niknejad, H. Fabrication of Nanochitosan Incorporated Polypyrrole/ Alginate Conducting Scaffold for Neural Tissue Engineering. Sci. Rep. 2020, 10, 22012.

(427) Muller, D.; Silva, J. P.; Rambo, C. R.; Barra, G. M. O.; Dourado, F.; Gama, F. M. Neuronal Cells Behavior on Polypyrrole Coated Bacterial Nanocellulose Three-Dimensional (3D) Scaffolds. J. Biomater. Sci., Polym. Ed. 2013, 24, 1368-1377.

(428) Sasso, L.; Vazquez, P.; Vedarethinam, I.; Castillo-León, J.; Emnéus, J.; Svendsen, W. E. Conducting Polymer 3D Microelectrodes. Sensors 2010, 10, 10986-11000.

(429) Thunberg, J.; Kalogeropoulos, T.; Kuzmenko, V.; Hägg, D.; Johannesson, S.; Westman, G.; Gatenholm, P. In Situ Synthesis of Conductive Polypyrrole on Electrospun Cellulose Nanofibers: Scaffold for Neural Tissue Engineering. Cellulose 2015, 22, 1459-1467.

(430) Gomez, N.; Lee, J. Y.; Nickels, J. D.; Schmidt, C. E. Micropatterned Polypyrrole: A Combination of Electrical and Topographical Characteristics for the Stimulation of Cells. Adv. Funct. Mater. 2007, 17, 1645-1653.

(431) Granato, A. E. C.; Ribeiro, A. C.; Marciano, F. R.; Rodrigues, B. V. M.; Lobo, A. O.; Porcionatto, M. Polypyrrole Increases Branching and Neurite Extension by Neuro2A Cells on PBAT Ultrathin Fibers. Nanomedicine 2018, 14, 1753-1763.

(432) Hernández-Ferrer, J.; Pérez-Bruzón, R. N.; Azanza, M. J.; González, M.; Del Moral, R.; Ansón-Casaos, A.; De La Fuente, J. M.; Marijuan, P. C.; Martínez, M. T. Study of Neuron Survival on Polypyrrole-Embedded Single-Walled Carbon Nanotube Substrates for Long-Term Growth Conditions. J. Biomed. Mater. Res., Part A 2014, 102, 4443-4454.

(433) Kim, S. Y.; Kim, K. M.; Hoffman-Kim, D.; Song, H. K.; Palmore, G. T. R. Quantitative Control of Neuron Adhesion at a Neural Interface 
Using a Conducting Polymer Composite with Low Electrical Impedance. ACS Appl. Mater. Interfaces 2011, 3, 16-21.

(434) Lee, J. Y.; Bashur, C. A.; Goldstein, A. S.; Schmidt, C. E. Polypyrrole-Coated Electrospun PLGA Nanofibers for Neural Tissue Applications. Biomaterials 2009, 30, 4325-4335.

(435) Abasi, S.; Aggas, J. R.; Guiseppi-Elie, A. Physiochemical and Morphological Dependent Growth of NIH/3T3 and PC-12 on Polyaniline-Chloride/Chitosan Bionanocomposites. Mater. Sci. Eng., C 2019, 99, 1304-1312.

(436) Bhang, S. H.; Jeong, S. I.; Lee, T. J.; Jun, I.; Lee, Y. B.; Kim, B. S.; Shin, H. Electroactive Electrospun Polyaniline/Poly[(L-Lactide)-Co( $\alpha$-Caprolactone)] Fibers for Control of Neural Cell Function. Macromol. Biosci. 2012, 12, 402-411.

(437) Cullen, D. K.; Patel, A. R.; Doorish, J. F.; Smith, D. H.; Pfister, B. J. Developing a Tissue-Engineered Neural-Electrical Relay Using Encapsulated Neuronal Constructs on Conducting Polymer Fibers. J. Neural Eng. 2008, 5, 374-384.

(438) Liu, S.; Wang, J.; Zhang, D.; Zhang, P.; Ou, J.; Liu, B.; Yang, S. Investigation on Cell Biocompatible Behaviors of Polyaniline Film Fabricated via Electroless Surface Polymerization. Appl. Surf. Sci. 2010, $256,3427-3431$.

(439) Oren, R.; Sfez, R.; Korbakov, N.; Shabtai, K.; Cohen, A.; Erez, H.; Dormann, A.; Cohen, H.; Shappir, J.; Spira, M. E.; et al. Electrically Conductive 2D-PAN-Containing Surfaces as a Culturing Substrate for Neurons. J. Biomater. Sci., Polym. Ed. 2004, 15, 1355-1374.

(440) Shrestha, B. K.; Shrestha, S.; Baral, E. R.; Lee, J. Y.; Kim, B. S.; Park, C. H.; Kim, C. S. П-Conjugated Polyaniline-Assisted Flexible Titania Nanotubes with Controlled Surface Morphology as Regenerative Medicine in Nerve Cell Growth. Chem. Eng. J. 2019, 360, 701713.

(441) Yang, H.; Zhu, G.; Huang, Y.; Shi, X.; Wang, Y. The Stimulation of the Differentiation of Pheochromocytoma (PC12-L) Cells into Neuron-like Cells by Electrically Conductive Nanofibre Mesh. Appl. Mater. Today 2016, 5, 215-222.

(442) Juarez-Hernandez, L. J.; Cornella, N.; Pasquardini, L.; Battistoni, S.; Vidalino, L.; Vanzetti, L.; Caponi, S.; Serra, M. D.; Iannotta, S.; Pederzolli, C.; et al. Bio-Hybrid Interfaces to Study Neuromorphic Functionalities: New Multidisciplinary Evidences of Cell Viability on Poly(Anyline) (PANI), a Semiconductor Polymer with Memristive Properties. Biophys. Chem. 2016, 208, 40-47.

(443) Bystrenova, E.; Jelitai, M.; Tonazzini, I.; Lazar, A. N.; Huth, M.; Stoliar, P.; Dionigi, C.; Cacace, M. G.; Nickel, B.; Madarasz, E.; Biscarini, F.; et al. Neural Networks Grown on Organic Semiconductors. Adv. Funct. Mater. 2008, 18, 1751-1756.

(444) Gattazzo, F.; De Maria, C.; Whulanza, Y.; Taverni, G.; Ahluwalia, A.; Vozzi, G. Realisation and Characterization of Conductive Hollow Fibers for Neuronal Tissue Engineering. J. Biomed. Mater. Res., Part B 2015, 103, 1107-1119.

(445) Bettinger, C. J.; Bruggeman, J. P.; Misra, A.; Borenstein, J. T.; Langer, R. S. Biocompatibility of Biodegradable Semiconducting Melanin Films for Nerve Tissue Engineering. Biomaterials 2009, 30, 3050-3057.

(446) Toffanin, S.; Benfenati, V.; Pistone, A.; Bonetti, S.; Koopman, W.; Posati, T.; Sagnella, A.; Natali, M.; Zamboni, R.; Ruani, G.; et al. NType Perylene-Based Organic Semiconductors for Functional Neural Interfacing. J. Mater. Chem. B 2013, 1, 3850-3859.

(447) Abidian, M. R.; Corey, J. M.; Kipke, D. R.; Martin, D. C. Conducting-Polymer Nanotubes Improve Electrical Properties, Mechanical Adhesion, Neural Attachment and Neurite Outgrowth of Neural Electrodes. Small 2010, 6, 421-429.

(448) Richardson-Burns, S. M.; Hendricks, J. L.; Foster, B.; Povlich, L. K.; Kim, D. H.; Martin, D. C. Polymerization of the Conducting Polymer Poly(3,4-Ethylenedioxythiophene) (PEDOT) around Living Neural Cells. Biomaterials 2007, 28, 1539-1552.

(449) Lu, C. H.; Hsiao, Y. S.; Kuo, C. W.; Chen, P. Electrically Tunable Organic Bioelectronics for Spatial and Temporal Manipulation of Neuron-like Pheochromocytoma (PC-12) Cells. Biochim. Biophys. Acta, Gen. Subj. 2013, 1830, 4321-4328.
(450) Fallahi, A.; Mandla, S.; Kerr-Phillip, T.; Seo, J.; Rodrigues, R. O.; Jodat, Y. A.; Samanipour, R.; Hussain, M. A.; Lee, C. K.; Bae, H.; et al. Flexible and Stretchable PEDOT-Embedded Hybrid Substrates for Bioengineering and Sensory Applications. ChemNanoMat 2019, 5, 729-737.

(451) Lei, Q.; He, J.; Li, D. Electrohydrodynamic 3D Printing of Layer-Specifically Oriented, Multiscale Conductive Scaffolds for Cardiac Tissue Engineering. Nanoscale 2019, 11, 15195-15205.

(452) Puiggalí-Jou, A.; Ordoño, J.; del Valle, L. J.; Pérez-Amodio, S.; Engel, E.; Alemán, C. Tuning Multilayered Polymeric Self-Standing Films for Controlled Release of L-Lactate by Electrical Stimulation. J. Controlled Release 2021, 330, 669-683.

(453) Stříteský, S.; Marková, A.; Víteček, J.; Safař́ková, E.; Hrabal, M.; Kubáć, L.; Kubala, L.; Weiter, M.; Vala, M. Printing Inks of Electroactive Polymer PEDOT:PSS: The Study of Biocompatibility, Stability, and Electrical Properties. J. Biomed. Mater. Res., Part A 2018, 106, 1121-1128.

(454) Kai, D.; Prabhakaran, M. P.; Jin, G.; Ramakrishna, S. Polypyrrole-Contained Electrospun Conductive Nanofibrous Membranes for Cardiac Tissue Engineering. J. Biomed. Mater. Res., Part A 2011, 99A, 376-385.

(455) Parchehbaf-Kashani, M.; Sepantafar, M.; Talkhabi, M.; Sayahpour, F. A.; Baharvand, H.; Pahlavan, S.; Rajabi, S. Design and Characterization of an Electroconductive Scaffold for Cardiomyocytes Based Biomedical Assays. Mater. Sci. Eng., C 2020, 109, 110603.

(456) Spearman, B. S.; Hodge, A. J.; Porter, J. L.; Hardy, J. G.; Davis, Z. D.; Xu, T.; Zhang, X.; Schmidt, C. E.; Hamilton, M. C.; Lipke, E. A. Conductive Interpenetrating Networks of Polypyrrole and Polycaprolactone Encourage Electrophysiological Development of Cardiac Cells. Acta Biomater. 2015, 28, 109-120.

(457) Tsui, J. H.; Ostrovsky-Snider, N. A.; Yama, D. M. P.; Donohue, J. D.; Choi, J. S.; Chavanachat, R.; Larson, J. D.; Murphy, A. R.; Kim, D. H. Conductive Silk-Polypyrrole Composite Scaffolds with Bioinspired Nanotopographic Cues for Cardiac Tissue Engineering. J. Mater. Chem. B 2018, 6, 7185-7196.

(458) Alves, T.; Souza, J.; Amaral, V.; Almeida, D.; Grotto, D.; Lima, R.; Aranha, N.; Silveira Filho, L.; Oliveira Junior, J.; Barros, C.; et al. Biomimetic Dense Lamellar Scaffold Based on a Colloidal Complex of the Polyaniline (PANi) and Biopolymers for Electroactive and Physiomechanical Stimulation of the Myocardial. Colloids Surf., A 2019, 579, 123650 .

(459) Baheiraei, N.; Yeganeh, H.; Ai, J.; Gharibi, R.; EbrahimiBarough, S.; Azami, M.; Vahdat, S.; Baharvand, H. Preparation of a Porous Conductive Scaffold from Aniline Pentamer-Modified Polyurethane/PCL Blend for Cardiac Tissue Engineering. J. Biomed. Mater. Res., Part A 2015, 103, 3179-3187.

(460) Bertuoli, P. T.; Ordono, J.; Armelin, E.; Pérez-Amodio, S.; Baldissera, A. F.; Ferreira, C. A.; Puiggalí, J.; Engel, E.; Del Valle, L. J.; Alemán, C. Electrospun Conducting and Biocompatible Uniaxial and Core-Shell Fibers Having Poly(Lactic Acid), Poly(Ethylene Glycol), and Polyaniline for Cardiac Tissue Engineering. ACS Omega 2019, 4, $3660-3672$.

(461) Wang, L.; Wu, Y.; Hu, T.; Guo, B.; Ma, P. X. Electrospun Conductive Nanofibrous Scaffolds for Engineering Cardiac Tissue and 3D Bioactuators. Acta Biomater. 2017, 59, 68-81.

(462) Šafaŕíková, E.; Švihálková Šindlerová, L.; Stříteský, S.; Kubala, L.; Vala, M.; Weiter, M.; Víteček, J. Evaluation and Improvement of Organic Semiconductors' Biocompatibility towards Fibroblasts and Cardiomyocytes. Sens. Actuators, B 2018, 260, 418-425.

(463) Maráková, N.; Boeva, Z. A.; Humpolícek, P.; Lindfors, T.; Pacherník, J.; Kašpárková, V.; Radaszkiewicz, K. A.; Capáková, Z.; Minařík, A.; Lehocký, M. Electrochemically Prepared Composites of Graphene Oxide and Conducting Polymers: Cytocompatibility of Cardiomyocytes and Neural Progenitors. Mater. Sci. Eng., C 2019, 105, 110029.

(464) Alba, N. A.; Du, Z. J.; Catt, K. A.; Kozai, T. D. Y.; Cui, X. T. In Vivo Electrochemical Analysis of a PEDOT/MWCNT Neural Electrode Coating. Biosensors 2015, 5, 618-646. 
(465) Catt, K.; Li, H.; Cui, X. T. Poly (3,4-Ethylenedioxythiophene) Graphene Oxide Composite Coatings for Controlling Magnesium Implant Corrosion. Acta Biomater. 2017, 48, 530-540.

(466) Saunier, V.; Flahaut, E.; Blatché, M. C.; Bergaud, C.; Maziz, A. Carbon Nanofiber-PEDOT Composite Films as Novel Microelectrode for Neural Interfaces and Biosensing. Biosens. Bioelectron. 2020, 165, 112413.

(467) Asplund, M.; Thaning, E.; Lundberg, J.; Sandberg-Nordqvist, A. C.; Kostyszyn, B.; Inganäs, O.; Von Holst, H. Toxicity Evaluation of PEDOT/Biomolecular Composites Intended for Neural Communication Electrodes. Biomed. Mater. 2009, 4, 045009.

(468) Chen, N.; Luo, B.; Patil, A. C.; Wang, J.; Gammad, G. G. L.; Yi, Z.; Liu, X.; Yen, S. C.; Ramakrishna, S.; Thakor, N. V. Nanotunnels within Poly(3,4-Ethylenedioxythiophene)-Carbon Nanotube Composite for Highly Sensitive Neural Interfacing. ACS Nano 2020, 14, 80598073.

(469) Du, Z. J.; Luo, X.; Weaver, C. L.; Cui, X. T. Poly(3,4Ethylenedioxythiophene)-Ionic Liquid Coating Improves Neural Recording and Stimulation Functionality of MEAs. J. Mater. Chem. C 2015, 3, 6515-6524.

(470) Marroquin, J. B.; Coleman, H. A.; Tonta, M. A.; Zhou, K.; Winther-Jensen, B.; Fallon, J.; Duffy, N. W.; Yan, E.; Abdulwahid, A. A.; Jasieniak, J. J.; et al. Neural Electrodes Based on 3D Organic Electroactive Microfibers. Adv. Funct. Mater. 2018, 28, 1700927.

(471) Kolarcik, C. L.; Luebben, S. D.; Sapp, S. A.; Hanner, J.; Snyder, N.; Kozai, T. D. Y.; Chang, E.; Nabity, J. A.; Nabity, S. T.; Lagenaur, C. F.; et al. Elastomeric and Soft Conducting Microwires for Implantable Neural Interfaces. Soft Matter 2015, 11, 4847-4861.

(472) Lu, Y.; Li, Y.; Pan, J.; Wei, P.; Liu, N.; Wu, B.; Cheng, J.; Lu, C.; Wang, L. Poly(3,4-Ethylenedioxythiophene)/Poly(Styrenesulfonate)Poly(Vinyl Alcohol)/Poly(Acrylic Acid) Interpenetrating Polymer Networks for Improving Optrode-Neural Tissue Interface in Optogenetics. Biomaterials 2012, 33, 378-394.

(473) Wang, K.; Tang, R.-Y.; Zhao, X.-B.; Li, J.-J.; Lang, Y.-R.; Jiang, X.-X.; Sun, H.-J.; Lin, Q.-X.; Wang, C.-Y. Covalent Bonding of YIGSR and RGD to PEDOT/PSS/MWCNT-COOH Composite Material to Improve the Neural Interface. Nanoscale 2015, 7, 18677-18685.

(474) Zhou, H.; Cheng, X.; Rao, L.; Li, T.; Duan, Y. Y. Poly(3,4Ethylenedioxythiophene)/Multiwall Carbon Nanotube Composite Coatings for Improving the Stability of Microelectrodes in Neural Prostheses Applications. Acta Biomater. 2013, 9, 6439-6449.

(475) George, P. M.; Lyckman, A. W.; Lavan, D. A.; Hegde, A.; Leung, Y.; Avasare, R.; Testa, C.; Alexander, P. M.; Langer, R.; Sur, M. Fabrication and Biocompatibility of Polypyrrole Implants Suitable for Neural Prosthetics. Biomaterials 2005, 26, 3511-3519.

(476) Lu, Y.; Li, T.; Zhao, X.; Li, M.; Cao, Y.; Yang, H.; Duan, Y. Y. Electrodeposited Polypyrrole/Carbon Nanotubes Composite Films Electrodes for Neural Interfaces. Biomaterials 2010, 31, 5169-5181.

(477) Xu, H.; Holzwarth, J. M.; Yan, Y.; Xu, P.; Zheng, H.; Yin, Y.; Li, S.; Ma, P. X. Conductive PPY/PDLLA Conduit for Peripheral Nerve Regeneration. Biomaterials 2014, 35, 225-235.

(478) Cui, Z.; Ni, N. C.; Wu, J.; Du, G. Q.; He, S.; Yau, T. M.; Weisel, R. D.; Sung, H. W.; Li, R. K. Polypyrrole-Chitosan Conductive Biomaterial Synchronizes Cardiomyocyte Contraction and Improves Myocardial Electrical Impulse Propagation. Theranostics 2018, 8, $2752-2764$.

(479) Wang, L.; Jiang, J.; Hua, W.; Darabi, A.; Song, X.; Song, C.; Zhong, W.; Xing, M. M. Q.; Qiu, X. Mussel-Inspired Conductive Cryogel as Cardiac Tissue Patch to Repair Myocardial Infarction by Migration of Conductive Nanoparticles. Adv. Funct. Mater. 2016, 26, $4293-4305$.

(480) Lei, T.; Guan, M.; Liu, J.; Lin, H. C.; Pfattner, R.; Shaw, L.; McGuire, A. F.; Huang, T. C.; Shao, L.; Cheng, K. T.; et al. Biocompatible and Totally Disintegrable Semiconducting Polymer for Ultrathin and Ultralightweight Transient Electronics. Proc. Natl. Acad. Sci. U. S. A. 2017, 114, 5107-5112.

(481) Chen, S.; Hsieh, M. H.; Li, S. H.; Wu, J.; Weisel, R. D.; Chang, Y.; Sung, H. W.; Li, R. K. A Conductive Cell-Delivery Construct as a Bioengineered Patch That Can Improve Electrical Propagation and
Synchronize Cardiomyocyte Contraction for Heart Repair. J. Controlled Release 2020, 320, 73-82.

(482) Zhang, C.; Hsieh, M. H.; Wu, S. Y.; Li, S. H.; Wu, J.; Liu, S. M.; Wei, H. J.; Weisel, R. D.; Sung, H. W.; Li, R. K. A Self-Doping Conductive Polymer Hydrogel That Can Restore Electrical Impulse Propagation at Myocardial Infarct to Prevent Cardiac Arrhythmia and Preserve Ventricular Function. Biomaterials 2020, 231, 119672.

(483) Kapnisi, M.; Mansfield, C.; Marijon, C.; Guex, A. G.; Perbellini, F.; Bardi, I.; Humphrey, E. J.; Puetzer, J. L.; Mawad, D.; Koutsogeorgis, D. C.; et al. Auxetic Cardiac Patches with Tunable Mechanical and Conductive Properties toward Treating Myocardial Infarction. Adv. Funct. Mater. 2018, 28, 1800618.

(484) Someya, T.; Bao, Z.; Malliaras, G. G. The Rise of Plastic Bioelectronics. Nature 2016, 540, 379-385.

(485) Owens, R. M.; Malliaras, G. G. Organic Electronics at the Interface with Biology. MRS Bull. 2010, 35, 449-456.

(486) Blau, A.; Murr, A.; Wolff, S.; Sernagor, E.; Medini, P.; Iurilli, G.; Ziegler, C.; Benfenati, F. Flexible, All-Polymer Microelectrode Arrays for the Capture of Cardiac and Neuronal Signals. Biomaterials 2011, 32, $1778-1786$

(487) Aqrawe, Z.; Wright, B.; Patel, N.; Vyas, Y.; Malmstrom, J.; Montgomery, J. M.; Williams, D.; Travas-Sejdic, J.; Svirskis, D. The Influence of Macropores on PEDOT/PSS Microelectrode Coatings for Neuronal Recording and Stimulation. Sens. Actuators, B 2019, 281, $549-560$.

(488) Zips, S.; Grob, L.; Rinklin, P.; Terkan, K.; Adly, N. Y.; Weiß, L. J. K.; Mayer, D.; Wolfrum, B. Fully Printed $\mu$-Needle Electrode Array from Conductive Polymer Ink for Bioelectronic Applications. ACS Appl. Mater. Interfaces 2019, 11, 32778-32786.

(489) Jones, P. D.; Moskalyuk, A.; Barthold, C.; Gutöhrlein, K.; Heusel, G.; Schröppel, B.; Samba, R.; Giugliano, M. Low-Impedance 3D PEDOT:PSS Ultramicroelectrodes. Front. Neurosci. 2020, 14, 405. (490) Wang, C.; Rubakhin, S. S.; Enright, M. J.; Sweedler, J. V.; Nuzzo, R. G. 3D Particle-Free Printing of Biocompatible Conductive Hydrogel Platforms for Neuron Growth and Electrophysiological Recording. Adv. Funct. Mater. 2021, 31, 2010246.

(491) Flachs, D.; Köhler, T.; Thielemann, C. Transparent Poly(3,4Ethylenedioxythiophene)-Based Microelectrodes for Extracellular Recording. Biointerphases 2018, 13, 041008.

(492) Koutsouras, D. A.; Hama, A.; Pas, J.; Gkoupidenis, P.; Hivert, B.; Faivre-Sarrailh, C.; Di Pasquale, E.; Owens, R. M.; Malliaras, G. G. PEDOT:PSS Microelectrode Arrays for Hippocampal Cell Culture Electrophysiological Recordings. MRS Commun. 2017, 7, 259-265.

(493) Kim, S. M.; Kim, N.; Kim, Y.; Baik, M. S.; Yoo, M.; Kim, D.; Lee, W. J.; Kang, D. H.; Kim, S.; Lee, K.; et al. High-Performance, PolymerBased Direct Cellular Interfaces for Electrical Stimulation and Recording. NPG Asia Mater. 2018, 10, 255-265.

(494) Charkhkar, H.; Knaack, G. L.; Mandal, H. S.; Keefer, E. W.; Pancrazio, J. J. Effects of Carbon Nanotube and Conducting Polymer Coated Microelectrodes on Single-Unit Recordings in Vitro. 2014 36th Annu. Int. Conf. IEEE Eng. Med. Biol. Soc. EMBC 2014 2014, 469-473. (495) Izsak, J.; Seth, H.; Andersson, M.; Vizlin-Hodzic, D.; Theiss, S.; Hanse, E.; Ågren, H.; Funa, K.; Illes, S. Robust Generation of PersonSpecific, Synchronously Active Neuronal Networks Using Purely Isogenic Human IPSC-3D Neural Aggregate Cultures. Front. Neurosci. 2019, 13, 351.

(496) Mandal, H. S.; Knaack, G. L.; Charkhkar, H.; McHail, D. G.; Kastee, J. S.; Dumas, T. C.; Peixoto, N.; Rubinson, J. F.; Pancrazio, J. J. Improving the Performance of Poly(3,4-Ethylenedioxythiophene) for Brain-Machine Interface Applications. Acta Biomater. 2014, 10, 24462454.

(497) Aqrawe, Z.; Patel, N.; Vyas, Y.; Bansal, M.; Montgomery, J.; Travas-Sejdic, J.; Svirskis, D. A Simultaneous Optical and Electrical InVitro Neuronal Recording System to Evaluate Microelectrode Performance. PLoS One 2020, 15, e0237709.

(498) Gerwig, R.; Fuchsberger, K.; Schroeppel, B.; Link, G. S.; Heusel, G.; Kraushaar, U.; Schuhmann, W.; Stett, A.; Stelzle, M. PEDOT-CNT Composite Microelectrodes for Recording and Electrostimulation 
Applications: Fabrication, Morphology, and Electrical Properties. Front. Neuroeng. 2012, 5, 8.

(499) Kumar, N.; Dougherty, J. A.; Manring, H. R.; Elmadbouh, I.; Mergaye, M.; Czirok, A.; Greta Isai, D.; Belevych, A. E.; Yu, L.; Janssen, P. M. L.; et al. Assessment of Temporal Functional Changes and MiRNA Profiling of Human IPSC-Derived Cardiomyocytes. Sci. Rep. 2019, 9, 13188.

(500) Choi, J. S.; Smith, A. S. T.; Williams, N. P.; Matsubara, T.; Choi, M.; Kim, J. W.; Kim, H. J.; Choi, S.; Kim, D. H. Nanopatterned Nafion Microelectrode Arrays for In Vitro Cardiac Electrophysiology. Adv. Funct. Mater. 2020, 30, 1910660.

(501) Stauffer, W. R.; Lau, P. M.; Bi, G. Q.; Cui, X. T. Rapid Modulation of Local Neural Activity by Controlled Drug Release from Polymer-Coated Recording Microelectrodes. J. Neural Eng. 2011, 8, 044001.

(502) Smith, A. S. T.; Choi, E.; Gray, K.; Macadangdang, J.; Ahn, E. H.; Clark, E. C.; Laflamme, M. A.; Wu, J. C.; Murry, C. E.; Tung, L.; et al. NanoMEA: A Tool for High-Throughput, Electrophysiological Phenotyping of Patterned Excitable Cells. Nano Lett. 2020, 20, 15611570.

(503) Heizmann, S.; Kilias, A.; Ruther, P.; Egert, U.; Asplund, M. Active Control of Dye Release for Neuronal Tracing Using PEDOTPSS Coated Electrodes. IEEE Trans. Neural Syst. Rehabil. Eng. 2018, 26, 299-306.

(504) Feiner, R.; Engel, L.; Fleischer, S.; Malki, M.; Gal, I.; Shapira, A.; Shacham-Diamand, Y.; Dvir, T. Engineered Hybrid Cardiac Patches with Multifunctional Electronics for Online Monitoring and Regulation of Tissue Function. Nat. Mater. 2016, 15, 679-685.

(505) Feiner, R.; Fleischer, S.; Shapira, A.; Kalish, O.; Dvir, T. Multifunctional Degradable Electronic Scaffolds for Cardiac Tissue Engineering. J. Controlled Release 2018, 281, 189-195.

(506) Lee, S.; Sasaki, D.; Kim, D.; Mori, M.; Yokota, T.; Lee, H.; Park, S.; Fukuda, K.; Sekino, M.; Matsuura, K.; et al. Ultrasoft Electronics to Monitor Dynamically Pulsing Cardiomyocytes. Nat. Nanotechnol. 2019, 14, 156-160.

(507) Demelas, M.; Lai, S.; Spanu, A.; Martinoia, S.; Cosseddu, P.; Barbaro, M.; Bonfiglio, A. Charge Sensing by Organic ChargeModulated Field Effect Transistors: Application to the Detection of Bio-Related Effects. J. Mater. Chem. B 2013, 1, 3811-3819.

(508) Spanu, A.; Lai, S.; Cosseddu, P.; Bonfiglio, A.; Tedesco, M.; Martinoia, S. Organic FET Device as a Novel Sensor for Cell Bioelectrical and Metabolic Activity Recordings. Int. IEEE/EMBS Conf. Neural Eng. NER 2013, 937-940.

(509) Spanu, A.; Tedesco, M.; Martinoia, S.; Lai, S.; Cosseddu, P.; Bonfiglio, A. Bioelectrical and Metabolic Activity Recordings by Means of Organic Field Effect Transistors. Proc. 2015 18th AISEM Annu. Conf. AISEM 2015 2015, 47-49.

(510) Spanu, A.; Tedesco, M. T.; Martines, L.; Martinoia, S.; Bonfiglio, A. An Organic Neurophysiological Tool for Neuronal Metabolic Activity Monitoring. APL Bioeng. 2018, 2, 046105.

(511) Hempel, F.; Law, J. K. Y.; Nguyen, T. C.; Munief, W.; Lu, X.; Pachauri, V.; Susloparova, A.; Vu, X. T.; Ingebrandt, S. PEDOT:PSS Organic Electrochemical Transistor Arrays for Extracellular Electrophysiological Sensing of Cardiac Cells. Biosens. Bioelectron. 2017, 93, 132-138.

(512) Cea, C.; Spyropoulos, G. D.; Jastrzebska-Perfect, P.; Ferrero, J. J.; Gelinas, J. N.; Khodagholy, D. Enhancement-Mode Ion-Based Transistor as a Comprehensive Interface and Real-Time Processing Unit for in Vivo Electrophysiology. Nat. Mater. 2020, 19, 679-686.

(513) Habib, A.; Zhu, X.; Can, U. I.; McLanahan, M. L.; Zorlutuna, P.; Yanik, A. A. Electro-Plasmonic Nanoantenna: A Nonfluorescent Optical Probe for Ultrasensitive Label-Free Detection of Electrophysiological Signals. Sci. Adv. 2019, 5, eaav9786.

(514) Santoro, F.; Van De Burgt, Y.; Keene, S. T.; Cui, B.; Salleo, A. Enhanced Cell-Chip Coupling by Rapid Femtosecond Laser Patterning of Soft PEDOT:PSS Biointerfaces. ACS Appl. Mater. Interfaces 2017, 9, 39116-39121.
(515) Kielar, M.; Gooch, H.; Xu, L.; Pandey, A. K.; Sah, P. Direct Detection of Neuronal Activity Using Organic Photodetectors. ACS Photonics 2021, 8, 228-237.

(516) Juzekaeva, E.; Nasretdinov, A.; Battistoni, S.; Berzina, T.; Iannotta, S.; Khazipov, R.; Erokhin, V.; Mukhtarov, M. Coupling Cortical Neurons through Electronic Memristive Synapse. Adv. Mater. Technol. 2019, 4, 1800350.

(517) Keene, S. T.; Lubrano, C.; Kazemzadeh, S.; Melianas, A.; Tuchman, Y.; Polino, G.; Scognamiglio, P.; Cinà, L.; Salleo, A.; van de Burgt, Y.; et al. A Biohybrid Synapse with Neurotransmitter-Mediated Plasticity. Nat. Mater. 2020, 19, 969-973.

(518) Matter, K.; Balda, M. S. Functional Analysis of Tight Junctions. Methods 2003, 30, 228-234.

(519) Förster, C. Tight Junctions and the Modulation of Barrier Function in Disease. Histochem. Cell Biol. 2008, 130, 55-70.

(520) Tang, V. W.; Goodenough, D. A. Paracellular Ion Channel at the Tight Junction. Biophys. J. 2003, 84, 1660-1673.

(521) Mullin, J. M. Epithelial Barriers, Compartmentation, and Cancer. Sci. STKE 2004, 2004, No. pe2.

(522) Asphahani, F.; Thein, M.; Veiseh, O.; Edmondson, D.; Kosai, R.; Veiseh, M.; Xu, J.; Zhang, M. Influence of Cell Adhesion and Spreading on Impedance Characteristics of Cell-Based Sensors. Biosens. Bioelectron. 2008, 23, 1307-1313.

(523) Bagnaninchi, P. O.; Drummond, N. Real-Time Label-Free Monitoring of Adipose-Derived Stem Cell Differentiation with Electric Cell-Substrate Impedance Sensing. Proc. Natl. Acad. Sci. U. S. A. 2011 , 108, 6462-6467.

(524) Gu, A. Y.; Kho, D. T.; Johnson, R. H.; Graham, E. S.; O'Carroll, S. J. In Vitro Wounding Models Using the Electric Cell-Substrate Impedance Sensing (ECIS)-Z $\theta$ Technology. Biosensors 2018, 8, 90.

(525) Pan, Y.; Hu, N.; Wei, X.; Gong, L.; Zhang, B.; Wan, H.; Wang, P. 3D Cell-Based Biosensor for Cell Viability and Drug Assessment by 3D Electric Cell/Matrigel-Substrate Impedance Sensing. Biosens. Bioelectron. 2019, 130, 344-351.

(526) Zitzmann, F. D.; Jahnke, H. G.; Nitschke, F.; Beck-Sickinger, A. G.; Abel, B.; Belder, D.; Robitzki, A. A. A Novel Microfluidic Microelectrode Chip for a Significantly Enhanced Monitoring of NPY-Receptor Activation in Live Mode. Lab Chip 2017, 17, 42944302.

(527) Schmid, Y. R. F.; Bürgel, S. C.; Misun, P. M.; Hierlemann, A.; Frey, O. Electrical Impedance Spectroscopy for Microtissue Spheroid Analysis in Hanging-Drop Networks. ACS Sensors 2016, 1, 1028-1035.

(528) Ateh, D. D.; Vadgama, P.; Navsaria, H. A. Culture of Human Keratinocytes on Polypyrrole-Based Conducting Polymers. Tissue Eng. 2006, 12, 645-655.

(529) Lin, P.; Yan, F.; Yu, J.; Chan, H. L. W.; Yang, M. The Application of Organic Electrochemical Transistors in Cell-Based Biosensors. Adv. Mater. 2010, 22, 3655-3660.

(530) Garcia-Hernando, M.; Saez, J.; Savva, A.; Basabe-Desmonts, L.; Owens, R. M.; Benito-Lopez, F. An Electroactive and ThermoResponsive Material for the Capture and Release of Cells. Biosens. Bioelectron. 2021, 191, 113405.

(531) Rivnay, J.; Leleux, P.; Hama, A.; Ramuz, M.; Huerta, M.; Malliaras, G. G.; Owens, R. M. Using White Noise to Gate Organic Transistors for Dynamic Monitoring of Cultured Cell Layers. Sci. Rep. 2015, 5, 11613.

(532) Jimison, L. H.; Tria, S. A.; Khodagholy, D.; Gurfinkel, M.; Lanzarini, E.; Hama, A.; Malliaras, G. G.; Owens, R. M. Measurement of Barrier Tissue Integrity with an Organic Electrochemical Transistor. Adv. Mater. 2012, 24, 5919-5923.

(533) Tria, S.; Jimison, L.; Hama, A.; Bongo, M.; Owens, R. Sensing of EGTA Mediated Barrier Tissue Disruption with an Organic Transistor. Biosensors 2013, 3, 44-57.

(534) Romeo, A.; Tarabella, G.; D’Angelo, P.; Caffarra, C.; Cretella, D.; Alfieri, R.; Petronini, P. G.; Iannotta, S. Drug-Induced Cellular Death Dynamics Monitored by a Highly Sensitive Organic Electrochemical System. Biosens. Bioelectron. 2015, 68, 791-797.

(535) Lingstedt, L. V.; Ghittorelli, M.; Brückner, M.; Reinholz, J.; Crăciun, N. I.; Torricelli, F.; Mailänder, V.; Gkoupidenis, P.; Blom, P. 
W. M. Monitoring of Cell Layer Integrity with a Current-Driven Organic Electrochemical Transistor. Adv. Healthcare Mater. 2019, 8, 1900128.

(536) Huerta, M.; Rivnay, J.; Ramuz, M.; Hama, A.; Owens, R. M. Early Detection of Nephrotoxicity In Vitro Using a Transparent Conducting Polymer Device. Appl. Vitr. Toxicol. 2016, 2, 17-25.

(537) Yeung, S. Y.; Gu, X.; Tsang, C. M.; Tsao, S. W. G.; Hsing, I.-m. Organic Electrochemical Transistor Array for Monitoring Barrier Integrity of Epithelial Cells Invaded by Nasopharyngeal Carcinoma. Sens. Actuators, B 2019, 297, 126761.

(538) Salyk, O.; Víteček, J.; Omasta, L.; Šafaříková, E.; Stříteský, S.; Vala, M.; Weiter, M. Organic Electrochemical Transistor Microplate for Real-Time Cell Culture Monitoring. Appl. Sci. 2017, 7, 998.

(539) Ferrell, N.; Desai, R. R.; Fleischman, A. J.; Roy, S.; Humes, H. D.; Fissell, W. H. A Microfluidic Bioreactor with Integrated Transepithelial Electrical Resistance (TEER) Measurement Electrodes for Evaluation of Renal Epithelial Cells. Biotechnol. Bioeng. 2010, 107, $707-716$.

(540) Yao, C.; Xie, C.; Lin, P.; Yan, F.; Huang, P.; Hsing, I.-M. Organic Electrochemical Transistor Array for Recording Transepithelial Ion Transport of Human Airway Epithelial Cells. Adv. Mater. 2013, 25, 6575-6580.

(541) Ramuz, M.; Hama, A.; Huerta, M.; Rivnay, J.; Leleux, P.; Owens, R. M. Combined Optical and Electronic Sensing of Epithelial Cells Using Planar Organic Transistors. Adv. Mater. 2014, 26, 70837090.

(542) Cicoira, F.; Sessolo, M.; Yaghmazadeh, O.; DeFranco, J. A.; Yang, S. Y.; Malliaras, G. G. Influence of Device Geometry on Sensor Characteristics of Planar Organic Electrochemical Transistors. Adv. Mater. 2010, 22, 1012-1016.

(543) Tria, S. A.; Ramuz, M.; Huerta, M.; Leleux, P.; Rivnay, J.; Jimison, L. H.; Hama, A.; Malliaras, G. G.; Owens, R. M. Dynamic Monitoring of Salmonella Typhimurium Infection of Polarized Epithelia Using Organic Transistors. Adv. Healthcare Mater. 2014, 3, $1053-1060$.

(544) Ferro, M. P.; Leclerc, L.; Sleiman, M.; Marchiori, B.; Pourchez, J.; Owens, R. M.; Ramuz, M. Effect of E Cigarette Emissions on Tracheal Cells Monitored at the Air-Liquid Interface Using an Organic Electrochemical Transistor. Adv. Biosyst. 2019, 3, 1800249.

(545) Decataldo, F.; Barbalinardo, M.; Gentili, D.; Tessarolo, M.; Calienni, M.; Cavallini, M.; Fraboni, B. Organic Electrochemical Transistors for Real-Time Monitoring of In Vitro Silver Nanoparticle Toxicity. Adv. Biosyst. 2020, 4, 1900204.

(546) Krauss, G.; Meichsner, F.; Hochgesang, A.; Mohanraj, J.; Salehi, S.; Schmode, P.; Thelakkat, M. Polydiketopyrrolopyrroles Carrying Ethylene Glycol Substituents as Efficient Mixed Ion-Electron Conductors for Biocompatible Organic Electrochemical Transistors. Adv. Funct. Mater. 2021, 31, 2010048.

(547) Diacci, C.; Lee, J. W.; Janson, P.; Dufil, G.; Méhes, G.; Berggren, M.; Simon, D. T.; Stavrinidou, E. Real-Time Monitoring of Glucose Export from Isolated Chloroplasts Using an Organic Electrochemical Transistor. Adv. Mater. Technol. 2020, 5, 1900262.

(548) Tarabella, G.; D’Angelo, P.; Cifarelli, A.; Dimonte, A.; Romeo, A.; Berzina, T.; Erokhin, V.; Iannotta, S. A Hybrid Living/Organic Electrochemical Transistor Based on the Physarum Polycephalum Cell Endowed with Both Sensing and Memristive Properties. Chem. Sci. 2015, 6, 2859-2868.

(549) Stavrinidou, E.; Gabrielsson, R.; Gomez, E.; Crispin, X.; Nilsson, O.; Simon, D. T.; Berggren, M. Electronic Plants. Sci. Adv. 2015, 1, e1501136.

(550) Mahat, M. M.; Sabere, A. S. M.; Azizi, J.; Amdan, N. A. N. Potential Applications of Conducting Polymers to Reduce Secondary Bacterial Infections among COVID-19 Patients: A Review. Emergent Mater. 2021, 4, 279-292.

(551) Löffler, S.; Libberton, B.; Richter-Dahlfors, A. Organic Bioelectronics in Infection. J. Mater. Chem. B 2015, 3, 4979-4992.

(552) Jeong, C. J.; Sharker, S. M.; In, I.; Park, S. Y. Iron Oxide@ PEDOT-Based Recyclable Photothermal Nanoparticles with Poly-
(Vinylpyrrolidone) Sulfobetaines for Rapid and Effective Antibacterial Activity. ACS Appl. Mater. Interfaces 2015, 7, 9469-9478.

(553) Enshaei, H.; Puiggalí-Jou, A.; del Valle, L. J.; Turon, P.; Saperas, N.; Alemán, C. Nanotheranostic Interface Based on Antibiotic-Loaded Conducting Polymer Nanoparticles for Real-Time Monitoring of Bacterial Growth Inhibition. Adv. Healthcare Mater. 2021, 10, 2001636.

(554) Molina, B. G.; Del Valle, L. J.; Turon, P.; Armelin, E.; Alemán, C. Electrochemical Sensor for Bacterial Metabolism Based on the Detection of NADH by Polythiophene Nanoparticles. J. Phys. Chem. C 2019, 123, 22181-22190.

(555) Méhes, G.; Roy, A.; Strakosas, X.; Berggren, M.; Stavrinidou, E.; Simon, D. T. Organic Microbial Electrochemical Transistor Monitoring Extracellular Electron Transfer. Adv. Sci. 2020, 7, 2000641.

(556) Da Silva, F. A. G., Jr.; Queiroz, J. C.; Macedo, E. R.; Fernandes, A. W. C.; Freire, N. B.; Da Costa, M. M.; De Oliveira, H. P. Antibacterial Behavior of Polypyrrole: The Influence of Morphology and Additives Incorporation. Mater. Sci. Eng., C 2016, 62, 317-322.

(557) Odaci, D.; Kiralp Kayahan, S.; Timur, S.; Toppare, L. Use of a Thiophene-Based Conducting Polymer in Microbial Biosensing. Electrochim. Acta 2008, 53, 4104-4108.

(558) Namvar, A.; Warriner, K. Microbial Imprinted Polypyrrole/ Poly(3-Methylthiophene) Composite Films for the Detection of Bacillus Endospores. Biosens. Bioelectron. 2007, 22, 2018-2024.

(559) Le, D. Q.; Takai, M.; Suekuni, S.; Tokonami, S.; Nishino, T.; Shiigi, H.; Nagaoka, T. Development of an Observation Platform for Bacterial Activity Using Polypyrrole Films Doped with Bacteria. Anal. Chem. 2015, 87, 4047-4052.

(560) Saito, M.; Ishiki, K.; Nguyen, D. Q.; Shiigi, H. A Microbial Platform Based on Conducting Polymers for Evaluating Metabolic Activity. Anal. Chem. 2019, 91, 12793-12798.

(561) Gomez-Carretero, S.; Libberton, B.; Svennersten, K.; Persson, K.; Jager, E.; Berggren, M.; Rhen, M.; Richter-Dahlfors, A. RedoxActive Conducting Polymers Modulate Salmonella Biofilm Formation by Controlling Availability of Electron Acceptors. npj Biofilms Microbiomes 2017, 3, 19.

(562) Sánchez-Jiménez, M.; Estrany, F.; Borràs, N.; Maiti, B.; Díaz Díaz, D.; Del Valle, L. J.; Alemán, C. Antimicrobial Activity of Poly(3,4Ethylenedioxythiophene) n-Doped with a Pyridinium-Containing Polyelectrolyte. Soft Matter 2019, 15, 7695-7703.

(563) Seshadri, D. T.; Bhat, N. V. Use of Polyaniline as an Antimicrobial Agent in Textiles. Indian J. Fibre Text. Res. 2005, 30, 204-206.

(564) Shi, N.; Guo, X.; Jing, H.; Gong, J.; Sun, C.; Yang, K. Antibacterial Effect of the Conducting Polyaniline. J. Mater. Sci. Technol. 2006, 22, 289-290.

(565) Gizdavic-Nikolaidis, M. R.; Bennett, J. R.; Swift, S.; Easteal, A. J.; Ambrose, M. Broad Spectrum Antimicrobial Activity of Functionalized Polyanilines. Acta Biomater. 2011, 7, 4204-4209.

(566) Varesano, A.; Vineis, C.; Aluigi, A.; Rombaldoni, F.; Tonetti, C.; Mazzuchetti, G. Antibacterial Efficacy of Polypyrrole in Textile Applications. Fibers Polym. 2013, 14, 36-42.

(567) Firoz Babu, K.; Dhandapani, P.; Maruthamuthu, S.; Anbu Kulandainathan, M. One Pot Synthesis of Polypyrrole Silver Nanocomposite on Cotton Fabrics for Multifunctional Property. Carbohydr. Polym. 2012, 90, 1557-1563.

(568) Liu, F.; Yuan, Y.; Li, L.; Shang, S.; Yu, X.; Zhang, Q.; Jiang, S.; $\mathrm{Wu}, \mathrm{Y}$. Synthesis of Polypyrrole Nanocomposites Decorated with Silver Nanoparticles with Electrocatalysis and Antibacterial Property. Composites, Part B 2015, 69, 232-236.

(569) Fujii, S.; Matsuzawa, S.; Hamasaki, H.; Nakamura, Y.; Bouleghlimat, A.; Buurma, N. J. Polypyrrole-Palladium Nanocomposite Coating of Micrometer-Sized Polymer Particles toward a Recyclable Catalyst. Langmuir 2012, 28, 2436-2447.

(570) Soleimani, M.; Ghorbani, M.; Salahi, S. Antibacterial Activity of Polypyrrole-Chitosan Nanocomposite: Mechanism of Action. Int. J. Nanosci. Nanotechnol. 2016, 12, 191-197.

(571) Salam, M. A.; Obaid, A. Y.; El-Shishtawy, R. M.; Mohamed, S. A. Synthesis of Nanocomposites of Polypyrrole/Carbon Nanotubes/ 
Silver Nano Particles and Their Application in Water Disinfection. RSC Adv. 2017, 7, 16878-16884.

(572) Salabat, A.; Mirhoseini, F.; Mahdieh, M.; Saydi, H. A Novel Nanotube-Shaped Polypyrrole-Pd Composite Prepared Using Reverse Microemulsion Polymerization and Its Evaluation as an Antibacterial Agent. New J. Chem. 2015, 39, 4109-4114.

(573) Baoukina, S.; Monticelli, L.; Tieleman, D. P. Interaction of Pristine and Functionalized Carbon Nanotubes with Lipid Membranes. J. Phys. Chem. B 2013, 117, 12113-12123.

(574) Ko, Y.; Kim, J.; Jeong, H. Y.; Kwon, G.; Kim, D.; Ku, M.; Yang, J.; Yamauchi, Y.; Kim, H. Y.; Lee, C.; et al. Antibacterial Poly (3,4Ethylenedioxythiophene):Poly(Styrene-Sulfonate)/Agarose Nanocomposite Hydrogels with Thermo-Processability and Self-Healing. Carbohydr. Polym. 2019, 203, 26-34.

(575) Le, D. Q.; Tokonami, S.; Nishino, T.; Shiigi, H.; Nagaoka, T. Electrochemical Evaluation of Poly(3,4-Ethylenedioxythiophene) Films Doped with Bacteria Based on Viability Analysis. Bioelectrochemistry 2015, 105, 50-55.

(576) Lee, B. S.; Lin, Y. C.; Hsu, W. C.; Hou, C. H.; Shyue, J. J.; Hsiao, S. Y.; Wu, P. J.; Lee, Y. Te; Luo, S. C. Engineering Antifouling and Antibacterial Stainless Steel for Orthodontic Appliances through Layerby-Layer Deposition of Nanocomposite Coatings. ACS Appl. Bio Mater. 2020, 3, 486-494.

(577) Yu, L.; Yan, X.; Ye, C.; Zhao, H.; Chen, X.; Hu, F.; Li, H. Bacterial Respiration and Growth Rates Affect the Feeding Preferences, Brood Size and Lifespan of Caenorhabditis Elegans. PLoS One 2015, 10, No. e0134401.

(578) Hrynkiewicz, K.; Baum, C.; Leinweber, P. Density, Metabolic Activity, and Identity of Cultivable Rhizosphere Bacteria on Salix Viminalis in Disturbed Arable and Landfill Soils. J. Plant Nutr. Soil Sci. 2010, 173, 747-756.

(579) Stokes, J. M.; Lopatkin, A. J.; Lobritz, M. A.; Collins, J. J. Bacterial Metabolism and Antibiotic Efficacy. Cell Metab. 2019, 30, 251-259.

(580) Song, R. B.; Wu, Y. C.; Lin, Z. Q.; Xie, J.; Tan, C. H.; Loo, J. S. C.; Cao, B.; Zhang, J. R.; Zhu, J. J.; Zhang, Q. Living and Conducting: Coating Individual Bacterial Cells with In Situ Formed Polypyrrole. Angew. Chem., Int. Ed. 2017, 56, 10516-10520.

(581) Li, C.; Ding, L.; Cui, H.; Zhang, L.; Xu, K.; Ren, H. Application of Conductive Polymers in Biocathode of Microbial Fuel Cells and Microbial Community. Bioresour. Technol. 2012, 116, 459-465.

(582) Przyborski, S. Technology Platforms for 3D Cell Culture; WileyBlackwell, 2017.

(583) Koledova, Z. 3D Cell Culture: An Introduction. Methods Mol. Biol. 2017, 1612, 1-11.

(584) Kapałczyńska, M.; Kolenda, T.; Przybyła, W.; Zajączkowska, M.; Teresiak, A.; Filas, V.; Ibbs, M.; Bliźniak, R.; Łuczewski, Ł.; Lamperska, K. 2D and 3D Cell Cultures - a Comparison of Different Types of Cancer Cell Cultures. Arch. Med. Sci. 2018, 14, 910-919.

(585) Duval, K.; Grover, H.; Han, L. H.; Mou, Y.; Pegoraro, A. F.; Fredberg, J.; Chen, Z. Modeling Physiological Events in 2D vs. 3D Cell Culture. Physiology 2017, 32, 266-277.

(586) Owens, R. M.; Iandolo, D.; Wittmann, K. A (Bio) Materials Approach to Three-Dimensional Cell Biology. MRS Commun. 2017, 7, 287-288.

(587) Bissell, M. J. Goodbye Flat Biology - Time for the 3rd and the 4th Dimensions. J. Cell Sci. 2017, 130, 3-5.

(588) Abbott, A. Biology's New Dimension. Nature 2003, 424, 870872.

(589) Chen, C. S. 3D Biomimetic Cultures: The Next Platform for Cell Biology. Trends Cell Biol. 2016, 26, 798-800.

(590) Alexander, F. A.; Price, D. T.; Bhansali, S. From Cellular Cultures to Cellular Spheroids: Is Impedance Spectroscopy a Viable Tool for Monitoring Multicellular Spheroid (MCS) Drug Models? IEEE Rev. Biomed. Eng. 2013, 6, 63-76.

(591) Alegret, N.; Dominguez-Alfaro, A.; Mecerreyes, D. 3D Scaffolds Based on Conductive Polymers for Biomedical Applications. Biomacromolecules 2019, 20, 73-89.
(592) Rogers, Z. J.; Zeevi, M. P.; Koppes, R.; Bencherif, S. A. Electroconductive Hydrogels for Tissue Engineering: Current Status and Future Perspectives. Bioelectricity 2020, 2, 279-292.

(593) Pyarasani, R. D.; Jayaramudu, T.; John, A. Polyaniline-Based Conducting Hydrogels. J. Mater. Sci. 2019, 54, 974-996.

(594) Oktay, S.; Alemdar, N. Electrically Controlled Release of 5Fluorouracil from Conductive Gelatin Methacryloyl-Based Hydrogels. J. Appl. Polym. Sci. 2019, 136, 46914.

(595) Paradee, N.; Thanokiang, J.; Sirivat, A. Conductive Poly(2Ethylaniline) Dextran-Based Hydrogels for Electrically Controlled Diclofenac Release. Mater. Sci. Eng., C 2021, 118, 111346.

(596) Puiggalí-Jou, A.; Cazorla, E.; Ruano, G.; Babeli, I.; Ginebra, M. P.; Garciá-Torres, J.; Alemán, C. Electroresponsive Alginate-Based Hydrogels for Controlled Release of Hydrophobic Drugs. ACS Biomater. Sci. Eng. 2020, 6, 6228-6240.

(597) De Paoli, M.-A.; Gazotti, W. A. Conductive Polymer Blends: Preparation, Properties and Applications. Macromol. Symp. 2002, 189, 83-104.

(598) Zhou, L.; Fan, L.; Yi, X.; Zhou, Z.; Liu, C.; Fu, R.; Dai, C.; Wang, Z.; Chen, X.; Yu, P.; et al. Soft Conducting Polymer Hydrogels CrossLinked and Doped by Tannic Acid for Spinal Cord Injury Repair. ACS Nano 2018, 12, 10957-10967.

(599) Moussa, M.; El-Kady, M. F.; Dubal, D.; Tung, T. T.; Nine, M. J.; Mohamed, N.; Kaner, R. B.; Losic, D. Self-Assembly and Cross-Linking of Conducting Polymers into 3D Hydrogel Electrodes for Supercapacitor Applications. ACS Appl. Energy Mater. 2020, 3, 923-932.

(600) Lu, B.; Yuk, H.; Lin, S.; Jian, N.; Qu, K.; Xu, J.; Zhao, X. Pure PEDOT:PSS Hydrogels. Nat. Commun. 2019, 10, 1043.

(601) Min, J. H.; Patel, M.; Koh, W. G. Incorporation of Conductive Materials into Hydrogels for Tissue Engineering Applications. Polymers 2018, 10, 1078

(602) Jin, Z. H.; Liu, Y. L.; Fan, W. T.; Huang, W. H. Integrating Flexible Electrochemical Sensor into Microfluidic Chip for Simulating and Monitoring Vascular Mechanotransduction. Small 2020, 16, 1903204.

(603) Shi, Z.; Gao, H.; Feng, J.; Ding, B.; Cao, X.; Kuga, S.; Wang, Y.; Zhang, L.; Cai, J. In Situ Synthesis of Robust Conductive Cellulose/ Polypyrrole Composite Aerogels and Their Potential Application in Nerve Regeneration. Angew. Chem., Int. Ed. 2014, 53, 5380-5384.

(604) Wang, L.; Hu, S.; Ullah, M. W.; Li, X.; Shi, Z.; Yang, G. Enhanced Cell Proliferation by Electrical Stimulation Based on Electroactive Regenerated Bacterial Cellulose Hydrogels. Carbohydr. Polym. 2020, 249, 116829.

(605) Spencer, A. R.; Primbetova, A.; Koppes, A. N.; Koppes, R. A.; Fenniri, H.; Annabi, N. Electroconductive Gelatin MethacryloylPEDOT:PSS Composite Hydrogels: Design, Synthesis, and Properties. ACS Biomater. Sci. Eng. 2018, 4, 1558-1567.

(606) Liu, R.; Zhang, S.; Zhao, C.; Yang, D.; Cui, T.; Liu, Y.; Min, Y. Regulated Surface Morphology of Polyaniline/Polylactic Acid Composite Nanofibers via Various Inorganic Acids Doping for Enhancing Biocompatibility in Tissue Engineering. Nanoscale Res. Lett. 2021, 16, 4.

(607) Xu, C.; Guan, S.; Wang, S.; Gong, W.; Liu, T.; Ma, X.; Sun, C. Biodegradable and Electroconductive Poly(3,4-Ethylenedioxythiophene)/Carboxymethyl Chitosan Hydrogels for Neural Tissue Engineering. Mater. Sci. Eng., C 2018, 84, 32-43.

(608) Xu, J.; Wong, C. W.; Hsu, S. H. An Injectable, Electroconductive Hydrogel/Scaffold for Neural Repair and Motion Sensing. Chem. Mater. 2020, 32, 10407-10422.

(609) Gong, H. Y.; Park, J.; Kim, W.; Kim, J.; Lee, J. Y.; Koh, W. G. A Novel Conductive and Micropatterned PEG-Based Hydrogel Enabling the Topographical and Electrical Stimulation of Myoblasts. ACS Appl. Mater. Interfaces 2019, 11, 47695-47706.

(610) Justin, G.; Guiseppi-Elie, A. Electroconductive Blends of Poly(HEMA-Co-PEGMA-Co-HMMAco-SPMA) and Poly(Py-CoPyBA): In Vitro Biocompatibility. J. Bioact. Compat. Polym. 2010, 25, $121-140$. 
(611) Agarwala, S. Electrically Conducting Hydrogels for Health Care: Concept, Fabrication Methods, and Applications. International Journal of Bioprinting 2020, 6, 273.

(612) Rastin, H.; Zhang, B.; Bi, J.; Hassan, K.; Tung, T. T.; Losic, D. 3D Printing of Cell-Laden Electroconductive Bioinks for Tissue Engineering Applications. J. Mater. Chem. B 2020, 8, 5862-5876.

(613) Distler, T.; Polley, C.; Shi, F.; Schneidereit, D.; Ashton, M. D.; Friedrich, O.; Kolb, J. F.; Hardy, J. G.; Detsch, R.; Seitz, H.; et al. Electrically Conductive and 3D-Printable Oxidized Alginate-Gelatin Polypyrrole:PSS Hydrogels for Tissue Engineering. Adv. Healthcare Mater. 2021, 10, 2001876.

(614) Chen, X.; Wu, Y.; Ranjan, V. D.; Zhang, Y. Three-Dimensional Electrical Conductive Scaffold from Biomaterial-Based Carbon Microfiber Sponge with Bioinspired Coating for Cell Proliferation and Differentiation. Carbon 2018, 134, 174-182.

(615) Solazzo, M.; O’Brien, F. J.; Nicolosi, V.; Monaghan, M. G. The Rationale and Emergence of Electroconductive Biomaterial Scaffolds in Cardiac Tissue Engineering. APL Bioengineering 2019, 3, 041501.

(616) Sikorski, P. Electroconductive Scaffolds for Tissue Engineering Applications. Biomater. Sci. 2020, 8, 5583-5588.

(617) Del Agua, I.; Marina, S.; Pitsalidis, C.; Mantione, D.; Ferro, M.; Iandolo, D.; Sanchez-Sanchez, A.; Malliaras, G. G.; Owens, R. M.; Mecerreyes, D. Conducting Polymer Scaffolds Based on Poly(3,4Ethylenedioxythiophene) and Xanthan Gum for Live-Cell Monitoring. ACS Omega 2018, 3, 7424-7431.

(618) Alegret, N.; Dominguez-Alfaro, A.; González-Domínguez, J. M.; Arnaiz, B.; Cossío, U.; Bosi, S.; Vázquez, E.; Ramos-Cabrer, P.; Mecerreyes, D.; Prato, M. Three-Dimensional Conductive Scaffolds as Neural Prostheses Based on Carbon Nanotubes and Polypyrrole. ACS Appl. Mater. Interfaces 2018, 10, 43904-43914.

(619) Dominguez-Alfaro, A.; Alegret, N.; Arnaiz, B.; GonzálezDomínguez, J. M.; Martin-Pacheco, A.; Cossío, U.; Porcarelli, L.; Bosi, S.; Vázquez, E.; Mecerreyes, D.; et al. Tailored Methodology Based on Vapor Phase Polymerization to Manufacture PEDOT/CNT Scaffolds for Tissue Engineering. ACS Biomater. Sci. Eng. 2020, 6, 1269-1278.

(620) Iandolo, D.; Pennacchio, F. A.; Mollo, V.; Rossi, D.; Dannhauser, D.; Cui, B.; Owens, R. M.; Santoro, F. Electron Microscopy for 3D Scaffolds-Cell Biointerface Characterization. Adv. Biosyst. 2019, 3, 1800103.

(621) Iandolo, D.; Ravichandran, A.; Liu, X.; Wen, F.; Chan, J. K. Y.; Berggren, M.; Teoh, S.-H.; Simon, D. T. Development and Characterization of Organic Electronic Scaffolds for Bone Tissue Engineering. Adv. Healthcare Mater. 2016, 5, 1505-1512.

(622) Zhao, W.; Li, Y.; Wu, S.; Wang, D.; Zhao, X.; Xu, F.; Zou, M.; Zhang, H.; He, X.; Cao, A. Highly Stable Carbon Nanotube/ Polyaniline Porous Network for Multifunctional Applications. ACS Appl. Mater. Interfaces 2016, 8, 34027-34033.

(623) Song, F.; Jie, W.; Zhang, T.; Li, W.; Jiang, Y.; Wan, L.; Liu, W.; Li, X.; Liu, B. Room-Temperature Fabrication of a Three-Dimensional Reduced-Graphene Oxide/Polypyrrole/Hydroxyapatite Composite Scaffold for Bone Tissue Engineering. RSC Adv. 2016, 6, 9280492812.

(624) Sajesh, K. M.; Jayakumar, R.; Nair, S. V.; Chennazhi, K. P. Biocompatible Conducting Chitosan/Polypyrrole-Alginate Composite Scaffold for Bone Tissue Engineering. Int. J. Biol. Macromol. 2013, 62, $465-471$.

(625) Fani, N.; Hajinasrollah, M.; Asghari Vostikolaee, M.; Baghaban Eslaminejad, M.; Mashhadiabbas, F.; Tongas, N.; Rasoulianboroujeni, M.; Yadegari, A.; Ede, K.; Tahriri, M.; et al. Influence of Conductive PEDOT:PSS in a Hard Tissue Scaffold: In Vitro and in Vivo Study. J. Bioact. Compat. Polym. 2019, 34, 436-441.

(626) Hesam Mahmoudinezhad, M.; Karkhaneh, A.; Jadidi, K. Effect of PEDOT:PSS in Tissue Engineering Composite Scaffold on Improvement and Maintenance of Endothelial Cell Function. J. Biosci. 2018, 43, 307-319.

(627) Yang, B.; Yao, F.; Ye, L.; Hao, T.; Zhang, Y.; Zhang, L.; Dong, D.; Fang, W.; Wang, Y.; Zhang, X.; et al. A Conductive PEDOT/ Alginate Porous Scaffold as a Platform to Modulate the Biological
Behaviors of Brown Adipose-Derived Stem Cells. Biomater. Sci. 2020, 8, $3173-3185$

(628) Lari, A.; Sun, T.; Sultana, N. PEDOT:PSS-Containing Nanohydroxyapatite/Chitosan Conductive Bionanocomposite Scaffold: Fabrication and Evaluation. J. Nanomater. 2016, 2016, 9421203.

(629) Zhao, X.; Li, P.; Guo, B.; Ma, P. X. Antibacterial and Conductive Injectable Hydrogels Based on Quaternized Chitosan-Graft-Polyaniline/Oxidized Dextran for Tissue Engineering. Acta Biomater. 2015, 26, $236-248$

(630) Lawal, A. T.; Wallace, G. G. Vapour Phase Polymerisation of Conducting and Non-Conducting Polymers: A Review. Talanta 2014, 119, 133-143.

(631) Park, J. S.; Kim, B.; Lee, B. T.; Choi, J. S.; Yim, J. H. Fabrication of an Electroconductive, Flexible, and Soft Poly(3,4-Ethylenedioxythiophene)-Thermoplastic Polyurethane Hybrid Scaffold by: In Situ Vapor Phase Polymerization. J. Mater. Chem. B 2018, 6, 4082-4088.

(632) Kim, S. W.; Lee, S. W.; Kim, J.; Yim, J. H.; Cho, K. Y. ThreeDimensional, High-Porosity Conducting Skeletal Structure from Biodegradable Microparticles with Vapor-Phase Polymerized Conformal Surface Layer. Polymer 2016, 102, 127-135.

(633) Choi, J. S.; Park, J. S.; Kim, B.; Lee, B. T.; Yim, J. H. In Vitro Biocompatibility of Vapour Phase Polymerised Conductive Scaffolds for Cell Lines. Polymer 2017, 124, 95-100.

(634) Solazzo, M.; Monaghan, M. G. Structural Crystallisation of Crosslinked 3D PEDOT:PSS Anisotropic Porous Biomaterials to Generate Highly Conductive Platforms for Tissue Engineering Applications. Biomater. Sci. 2021, 9, 4317.

(635) Tang, X.; Saveh-Shemshaki, N.; Kan, H. M.; Khan, Y.; Laurencin, C. T. Biomimetic Electroconductive Nanofibrous Matrices for Skeletal Muscle Regenerative Engineering. Regen. Eng. Transl. Med. 2020, 6, 228-237.

(636) Bolin, M. H.; Svennersten, K.; Wang, X.; Chronakis, I. S.; Richter-Dahlfors, A.; Jager, E. W. H.; Berggren, M. Nano-Fiber Scaffold Electrodes Based on PEDOT for Cell Stimulation. Sens. Actuators, B 2009, 142, 451-456.

(637) Chen, C.; Yu, Y.; Li, K.; Zhao, M.; Liu, L.; Yang, J.; Liu, J.; Sun, D. Facile Approach to the Fabrication of 3D Electroconductive Nanofibers with Controlled Size and Conductivity Templated by Bacterial Cellulose. Cellulose 2015, 22, 3929-3939.

(638) Yow, S.-Z.; Lim, T. H.; Yim, E. K. F.; Lim, C. T.; Leong, K. W. A 3D Electroactive Polypyrrole-Collagen Fibrous Scaffold for Tissue Engineering. Polymers (Basel, Switz.) 2011, 3, 527-544.

(639) Chen, J.; Yu, M.; Guo, B.; Ma, P. X.; Yin, Z. Conductive Nanofibrous Composite Scaffolds Based on In-Situ Formed Polyaniline Nanoparticle and Polylactide for Bone Regeneration. J. Colloid Interface Sci. 2018, 514, 517-527.

(640) Guex, A. G.; Spicer, C. D.; Armgarth, A.; Gelmi, A.; Humphrey, E. J.; Terracciano, C. M.; Harding, S. E.; Stevens, M. M. Electrospun Aniline-Tetramer-Co-Polycaprolactone Fibers for Conductive, Biodegradable Scaffolds. MRS Commun. 2017, 7, 375-382.

(641) Garrudo, F. F. F.; Mikael, P. E.; Rodrigues, C. A. V.; Udangawa, R. W.; Paradiso, P.; Chapman, C. A.; Hoffman, P.; Colaço, R.; Cabral, J. M. S.; Morgado, J.; et al. Polyaniline-Polycaprolactone Fibers for Neural Applications: Electroconductivity Enhanced by PseudoDoping. Mater. Sci. Eng., C 2021, 120, 111680.

(642) Xue, J.; Wu, T.; Dai, Y.; Xia, Y. Electrospinning and Electrospun Nanofibers: Methods, Materials, and Applications. Chem. Rev. 2019, 119,5298

(643) Wang, W.; Ouaras, K.; Rutz, A. L.; Li, X.; Gerigk, M.; Naegele, T. E.; Malliaras, G. G.; Huang, Y. Y. S. Inflight Fiber Printing toward Array and 3D Optoelectronic and Sensing Architectures. Sci. Adv. 2020, 6, No. eaba0931.

(644) Babaie, A.; Bakhshandeh, B.; Abedi, A.; Mohammadnejad, J.; Shabani, I.; Ardeshirylajimi, A.; Reza Moosavi, S.; Amini, J.; Tayebi, L. Synergistic Effects of Conductive PVA/PEDOT Electrospun Scaffolds and Electrical Stimulation for More Effective Neural Tissue Engineering. Eur. Polym. J. 2020, 140, 110051. 
(645) Abedi, A.; Hasanzadeh, M.; Tayebi, L. Conductive Nanofibrous Chitosan/PEDOT:PSS Tissue Engineering Scaffolds. Mater. Chem. Phys. 2019, 237, 121882.

(646) Sarvari, R.; Agbolaghi, S.; Beygi-Khosrowshahi, Y.; Massoumi, B. Towards Skin Tissue Engineering Using Poly(2-Hydroxy Ethyl Methacrylate)-Co-Poly(N-Isopropylacrylamide)-Co-Poly $(\alpha$-Caprolactone) Hydrophilic Terpolymers. Int. J. Polym. Mater. 2019, 68, 691700.

(647) Sarvari, R.; Akbari-Alanjaraghi, M.; Massoumi, B.; BeygiKhosrowshahi, Y.; Agbolaghi, S. Conductive and Biodegradable Scaffolds Based on a Five-Arm and Functionalized Star-like Polyaniline-Polycaprolactone Copolymer with a d-Glucose Core. New J. Chem. 2017, 41, 6371-6384.

(648) Zhu, R.; Sun, Z.; Li, C.; Ramakrishna, S.; Chiu, K.; He, L. Electrical Stimulation Affects Neural Stem Cell Fate and Function in Vitro. Exp. Neurol. 2019, 319, 112963.

(649) Prabhakaran, M. P.; Ghasemi-Mobarakeh, L.; Jin, G.; Ramakrishna, S. Electrospun Conducting Polymer Nanofibers and Electrical Stimulation of Nerve Stem Cells. J. Biosci. Bioeng. 2011, 112, 501-507.

(650) Daraeinejad, Z.; Shabani, I. Enhancing Biocompatibility of Polyaniline-Based Scaffolds by Using a Bioactive Dopant. Synth. Met. 2021, 271, 116642.

(651) Das, S.; Sharma, M.; Saharia, D.; Sarma, K. K.; Muir, E. M.; Bora, U. Electrospun Silk-Polyaniline Conduits for Functional Nerve Regeneration in Rat Sciatic Nerve Injury Model. Biomed. Mater. 2017, 12,045025 .

(652) Khalili Amand, F.; Esmaeili, A. Investigating the Properties of Electrospun Nanofibers Made of Hybride Polymer Containing Anticoagulant Drugs. Carbohydr. Polym. 2020, 228, 115397.

(653) Li, Y.; Li, X.; Zhao, R.; Wang, C.; Qiu, F.; Sun, B.; Ji, H.; Qiu, J.; Wang, C. Enhanced Adhesion and Proliferation of Human Umbilical Vein Endothelial Cells on Conductive PANI-PCL Fiber Scaffold by Electrical Stimulation. Mater. Sci. Eng., C 2017, 72, 106-112.

(654) Garrudo, F. F. F.; Chapman, C. A.; Hoffman, P. R.; Udangawa, R. W.; Silva, J. C.; Mikael, P. E.; Rodrigues, C. A. V.; Cabral, J. M. S.; Morgado, J. M. F.; Ferreira, F. C.; et al. Polyaniline-Polycaprolactone Blended Nanofibers for Neural Cell Culture. Eur. Polym. J. 2019, 117, $28-37$.

(655) Rasti Boroojeni, F.; Mashayekhan, S.; Abbaszadeh, H. A.; Ansarizadeh, M.; Khoramgah, M. S.; Movaghar, V. R. Bioinspired Nanofiber Scaffold for Differentiating Bone Marrow-Derived Neural Stem Cells to Oligodendrocyte-like Cells: Design, Fabrication, and Characterization. Int. J. Nanomed. 2020, 15, 3903-3920.

(656) Rezk, A. I.; Bhattarai, D. P.; Park, J.; Park, C. H.; Kim, C. S. Polyaniline-Coated Titanium Oxide Nanoparticles and SimvastatinLoaded Poly $(\alpha$-Caprolactone) Composite Nanofibers Scaffold for Bone Tissue Regeneration Application. Colloids Surf., B 2020, 192, 111007.

(657) Gähwiler, B. H. Organotypic Cultures of Neural Tissue. Trends Neurosci. 1988, 11, 484-489.

(658) Shamir, E. R.; Ewald, A. J. Three-Dimensional Organotypic Culture: Experimental Models of Mammalian Biology and Disease. Nat. Rev. Mol. Cell Biol. 2014, 15, 647-664.

(659) Huerta, M.; Rivnay, J.; Ramuz, M.; Hama, A.; Owens, R. M. Research Update: Electrical Monitoring of Cysts Using Organic Electrochemical Transistors. APL Mater. 2015, 3, 030701.

(660) Curto, V. F.; Ferro, M. P.; Mariani, F.; Scavetta, E.; Owens, R. M. A Planar Impedance Sensor for 3D Spheroids. Lab Chip 2018, 18, 933-943.

(661) Lancaster, M. A.; Huch, M. Disease Modelling in Human Organoids. Dis. Models \&amp; Mech. 2019, 12, No. dmm039347.

(662) Dutta, D.; Heo, I.; Clevers, H. Disease Modeling in Stem CellDerived 3D Organoid Systems. Trends Mol. Med. 2017, 23, 393-410.

(663) Kim, J.; Koo, B. K.; Knoblich, J. A. Human Organoids: Model Systems for Human Biology and Medicine. Nat. Rev. Mol. Cell Biol. 2020, 21, 571-584.

(664) Clevers, H. Modeling Development and Disease with Organoids. Cell 2016, 165, 1586-1597.
(665) Kalmykov, A.; Huang, C.; Bliley, J.; Shiwarski, D.; Tashman, J.; Abdullah, A.; Rastogi, S. K.; Shukla, S.; Mataev, E.; Feinberg, A. W.; et al. Organ-on-e-Chip: Three-Dimensional Self-Rolled Biosensor Array for Electrical Interrogations of Human Electrogenic Spheroids. Sci. Adv. 2019, 5, eaax0729.

(666) Galvani, L. De Viribus Electricitatis in Motu Musculari Commentarius 1791, 7, 363-418.

(667) Levin, M. Molecular Bioelectricity: How Endogenous Voltage Potentials Control Cell Behavior and Instruct Pattern Regulation in Vivo. Mol. Biol. Cell 2014, 25, 3835-3850.

(668) Levin, M.; Selberg, J.; Rolandi, M. Endogenous Bioelectrics in Development, Cancer, and Regeneration: Drugs and Bioelectronic Devices as Electroceuticals for Regenerative Medicine. iScience 2019, 22, 519-533.

(669) Sundelacruz, S.; Levin, M.; Kaplan, D. L. Membrane Potential Controls Adipogenic and Osteogenic Differentiation of Mesenchymal Stem Cells. PLoS One 2008, 3, e3737.

(670) Sundelacruz, S.; Moody, A. T.; Levin, M.; Kaplan, D. L. Membrane Potential Depolarization Alters Calcium Flux and Phosphate Signaling During Osteogenic Differentiation of Human Mesenchymal Stem Cells. Bioelectricity 2019, 1, 56-66.

(671) Bicer, M.; Sheard, J.; Iandolo, D.; Boateng, S. Y.; Cottrell, G. S.; Widera, D. Electrical Stimulation of Adipose-Derived Stem Cells in 3D Nanofibrillar Cellulose Increases Their Osteogenic Potential. Biomolecules 2020, 10, 1696.

(672) Kwon, H. J.; Lee, G. S.; Chun, H. Electrical Stimulation Drives Chondrogenesis of Mesenchymal Stem Cells in the Absence of Exogenous Growth Factors. Sci. Rep. 2016, 6, 39302.

(673) Vadlamani, R. A.; Nie, Y.; Detwiler, D. A.; Dhanabal, A.; Kraft, A. M.; Kuang, S.; Gavin, T. P.; Garner, A. L. Nanosecond Pulsed Electric Field Induced Proliferation and Differentiation of Osteoblasts and Myoblasts. J. R. Soc., Interface 2019, 16, 20190079.

(674) Li, K.; Ning, T.; Wang, H.; Jiang, Y.; Zhang, J.; Ge, Z. Nanosecond Pulsed Electric Fields Enhance Mesenchymal Stem Cells Differentiation via DNMT1-Regulated OCT4/NANOG Gene Expression. Stem Cell Res. Ther. 2020, 11, 308.

(675) Petty, A. J.; Keate, R. L.; Jiang, B.; Ameer, G. A.; Rivnay, J. Conducting Polymers for Tissue Regeneration in Vivo $†$. Chem. Mater. 2020, 32, 4095-4115.

(676) da Silva, L. P.; Kundu, S. C.; Reis, R. L.; Correlo, V. M. Electric Phenomenon: A Disregarded Tool in Tissue Engineering and Regenerative Medicine. Trends Biotechnol. 2020, 38, 24-49.

(677) Lee, S.; Ozlu, B.; Eom, T.; Martin, D. C.; Shim, B. S. Electrically Conducting Polymers for Bio-Interfacing Electronics: From Neural and Cardiac Interfaces to Bone and Artificial Tissue Biomaterials. Biosens. Bioelectron. 2020, 170, 112620.

(678) Hsiao, Y. S.; Lin, C. C.; Hsieh, H. J.; Tsai, S. M.; Kuo, C. W.; Chu, C. W.; Chen, P. Manipulating Location, Polarity, and Outgrowth Length of Neuron-like Pheochromocytoma (PC-12) Cells on Patterned Organic Electrode Arrays. Lab Chip 2011, 11, 3674-3680.

(679) Chen, X.; Liu, C.; Huang, Z.; Pu, X.; Shang, L.; Yin, G.; Xue, C. Preparation of Carboxylic Graphene Oxide-Composited Polypyrrole Conduits and Their Effect on Sciatic Nerve Repair under Electrical Stimulation. J. Biomed. Mater. Res., Part A 2019, 107, 2784-2795.

(680) Gomez, N.; Schmidt, C. E. Nerve Growth Factor-Immobilized Polypyrrole: Bioactive Electrically Conducting Polymer for Enhanced Neurite Extension. J. Biomed. Mater. Res., Part A 2007, 81A, 135-149. (681) Kim, S.; Jang, L. K.; Jang, M.; Lee, S.; Hardy, J. G.; Lee, J. Y. Electrically Conductive Polydopamine-Polypyrrole as High Performance Biomaterials for Cell Stimulation in Vitro and Electrical Signal Recording in Vivo. ACS Appl. Mater. Interfaces 2018, 10, 33032-33042. (682) Liu, C.; Huang, Z.; Pu, X.; Shang, L.; Yin, G.; Chen, X.; Cheng, S. Fabrication of Carboxylic Graphene Oxide-Composited Polypyrrole Film for Neurite Growth under Electrical Stimulation. Front. Mater. Sci. 2019, 13, 258-267.

(683) Zeng, J.; Huang, Z.; Yin, G.; Qin, J.; Chen, X.; Gu, J. Fabrication of Conductive NGF-Conjugated Polypyrrole-Poly(l-Lactic Acid) Fibers and Their Effect on Neurite Outgrowth. Colloids Surf., B 2013, $110,450-457$. 
(684) Zhou, X.; Yang, A.; Huang, Z.; Yin, G.; Pu, X.; Jin, J. Enhancement of Neurite Adhesion, Alignment and Elongation on Conductive Polypyrrole-Poly(Lactide Acid) Fibers with Cell-Derived Extracellular Matrix. Colloids Surf., B 2017, 149, 217-225.

(685) Zhang, J.; Qiu, K.; Sun, B.; Fang, J.; Zhang, K.; Ei-Hamshary, H.; Al-Deyab, S. S.; Mo, X. The Aligned Core-Sheath Nanofibers with Electrical Conductivity for Neural Tissue Engineering. J. Mater. Chem. B 2014, 2, 7945-7954.

(686) Sun, B.; Wu, T.; Wang, J.; Li, D.; Wang, J.; Gao, Q.; Bhutto, M. A.; El-Hamshary, H.; Al-Deyab, S. S.; Mo, X. Polypyrrole-Coated Poly(1-Lactic Acid-: Co - $\epsilon$-Caprolactone)/Silk Fibroin Nanofibrous Membranes Promoting Neural Cell Proliferation and Differentiation with Electrical Stimulation. J. Mater. Chem. B 2016, 4, 6670-6679.

(687) Wang, S.; Guan, S.; Wang, J.; Liu, H.; Liu, T.; Ma, X.; Cui, Z. Fabrication and Characterization of Conductive Poly (3,4-Ethylenedioxythiophene) Doped with Hyaluronic Acid/Poly (L-Lactic Acid) Composite Film for Biomedical Application. J. Biosci. Bioeng. 2017, 123, 116-125.

(688) Jing, W.; Zhang, Y.; Cai, Q.; Chen, G.; Wang, L.; Yang, X.; Zhong, W. Study of Electrical Stimulation with Different Electric-Field Intensities in the Regulation of the Differentiation of PC12 Cells. ACS Chem. Neurosci. 2019, 10, 348-357.

(689) Heo, D. N.; Lee, S. J.; Timsina, R.; Qiu, X.; Castro, N. J.; Zhang, L. G. Development of 3D Printable Conductive Hydrogel with Crystallized PEDOT:PSS for Neural Tissue Engineering. Mater. Sci. Eng., C 2019, 99, 582-590.

(690) Rajnicek, A. M.; Zhao, Z.; Moral-Vico, J.; Cruz, A. M.; McCaig, C. D.; Casañ-Pastor, N. Controlling Nerve Growth with an Electric Field Induced Indirectly in Transparent Conductive Substrate Materials. Adv. Healthcare Mater. 2018, 7, 1800473.

(691) Ho, D.; Zou, J.; Chen, X.; Munshi, A.; Smith, N. M.; Agarwal, V.; Hodgetts, S. I.; Plant, G. W.; Bakker, A. J.; Harvey, A. R.; et al. Hierarchical Patterning of Multifunctional Conducting Polymer Nanoparticles as a Bionic Platform for Topographic Contact Guidance. ACS Nano 2015, 9, 1767-1774.

(692) Bhattarai, D. P.; Hwang, T. I.; Kim, J. I.; Lee, J. H.; Chun, S.; Kim, B. S.; Park, C. H.; Kim, C. S. Synthesis of Polypyrrole Nanorods via Sacrificial Removal of Aluminum Oxide Nanopore Template: A Study on Cell Viability, Electrical Stimulation and Neuronal Differentiation of PC12 cells. Mater. Sci. Eng., C 2020, 107, 110325.

(693) Shrestha, S.; Shrestha, B. K.; Joong, O. K.; Park, C. H.; Kim, C. S. Para -Substituted Sulfonic Acid-Doped Protonated Emeraldine Salt Nanobuds: A Potent Neural Interface Targeting PC12 Cell Interactions and Promotes Neuronal Cell Differentiation. Biomater. Sci. 2021, 9, 1691-1704.

(694) Elashnikov, R.; Rimpelová, S.; Děkanovský, L.; Svorčík, V.; Lyutakov, O. Polypyrrole-Coated Cellulose Nanofibers: Influence of Orientation, Coverage and Electrical Stimulation on SH-SY5Y Behavior. J. Mater. Chem. B 2019, 7, 6500-6507.

(695) Moroder, P.; Runge, M. B.; Wang, H.; Ruesink, T.; Lu, L.; Spinner, R. J.; Windebank, A. J.; Yaszemski, M. J. Material Properties and Electrical Stimulation Regimens of Polycaprolactone FumaratePolypyrrole Scaffolds as Potential Conductive Nerve Conduits. Acta Biomater. 2011, 7, 944-953.

(696) Zheng, Z.; Huang, L.; Yan, L.; Yuan, F.; Wang, L.; Wang, K.; Lawson, T.; Lin, M.; Liu, Y. Polyaniline Functionalized Graphene Nanoelectrodes for the Regeneration of PC12 Cells via Electrical Stimulation. Int. J. Mol. Sci. 2019, 20, 2013.

(697) Tsai, N. C.; She, J. W.; Wu, J. G.; Chen, P.; Hsiao, Y. S.; Yu, J. Poly(3,4-Ethylenedioxythiophene) Polymer Composite Bioelectrodes with Designed Chemical and Topographical Cues to Manipulate the Behavior of PC12 Neuronal Cells. Adv. Mater. Interfaces 2019, 6, 1801576.

(698) Bonisoli, A.; Marino, A.; Ciofani, G.; Greco, F. Topographical and Electrical Stimulation of Neuronal Cells through Microwrinkled Conducting Polymer Biointerfaces. Macromol. Biosci. 2017, 17, 1700128.

(699) Yan, L.; Zhao, B.; Liu, X.; Li, X.; Zeng, C.; Shi, H.; Xu, X.; Lin, T.; Dai, L.; Liu, Y. Aligned Nanofibers from Polypyrrole/Graphene as
Electrodes for Regeneration of Optic Nerve via Electrical Stimulation. ACS Appl. Mater. Interfaces 2016, 8, 6834-6840.

(700) Zhang, Q.; Esrafilzadeh, D.; Crook, J. M.; Kapsa, R.; Stewart, E. M.; Tomaskovic-Crook, E.; Wallace, G. G.; Huang, X. F. Electrical Stimulation Using Conductive Polymer Polypyrrole Counters Reduced Neurite Outgrowth of Primary Prefrontal Cortical Neurons from NRG1-KO and DISC1-LI Mice. Sci. Rep. 2017, 7, 42525.

(701) Reddy, S.; Xiao, Q.; Liu, H.; Li, C.; Chen, S.; Wang, C.; Chiu, K.; Chen, N.; Tu, Y.; Ramakrishna, S.; et al. Bionanotube/Poly (3,4Ethylenedioxythiophene) Nanohybrid as an Electrode for the Neural Interface and Dopamine Sensor. ACS Appl. Mater. Interfaces 2019, 11, 18254-18267.

(702) Nguyen, H. T.; Sapp, S.; Wei, C.; Chow, J. K.; Nguyen, A.; Coursen, J.; Luebben, S.; Chang, E.; Ross, R.; Schmidt, C. E. Electric Field Stimulation through a Biodegradable Polypyrrole-Co- Polycaprolactone Substrate Enhances Neural Cell Growth. J. Biomed. Mater. Res., Part A 2014, 102, 2554-2564.

(703) Shannon, R. V. A Model of Safe Levels for Electrical Stimulation. IEEE Trans. Biomed. Eng. 1992, 39, 424-426.

(704) Nagamine, K.; Hirata, T.; Okamoto, K.; Abe, Y.; Kaji, H.; Nishizawa, M. Portable Micropatterns of Neuronal Cells Supported by Thin Hydrogel Films. ACS Biomater. Sci. Eng. 2015, 1, 329-334.

(705) Nyberg, T.; Shimada, A.; Torimitsu, K. Ion Conducting Polymer Microelectrodes for Interfacing with Neural Networks. J. Neurosci. Methods 2007, 160, 16-25.

(706) Evans, A. J.; Thompson, B. C.; Wallace, G. G.; Millard, R.; O'Leary, S. J.; Clark, G. M.; Shepherd, R. K.; Richardson, R. T. Promoting Neurite Outgrowth from Spiral Ganglion Neuron Explants Using Polypyrrole/BDNF-Coated Electrodes. J. Biomed. Mater. Res., Part A 2009, 91A, 241-250.

(707) Rong, G.; Kim, E. H.; Qiang, Y.; Di, W.; Zhong, Y.; Zhao, X.; Fang, H.; Clark, H. A. Imaging Sodium Flux during Action Potentials in Neurons with Fluorescent Nanosensors and Transparent Microelectrodes. ACS Sensors 2018, 3, 2499-2505.

(708) Kim, R.; Nam, Y. Polydopamine-Doped Conductive Polymer Microelectrodes for Neural Recording and Stimulation. J. Neurosci. Methods 2019, 326, 108369.

(709) Ostrovsky, S.; Hahnewald, S.; Kiran, R.; Mistrik, P.; Hessler, R.; Tscherter, A.; Senn, P.; Kang, J.; Kim, J.; Roccio, M.; et al. Conductive Hybrid Carbon Nanotube (CNT)-Polythiophene Coatings for Innovative Auditory Neuron-Multi-Electrode Array Interfacing. RSC $A d v$. 2016, 6, 41714-41723.

(710) Zhang, Q.; Beirne, S.; Shu, K.; Esrafilzadeh, D.; Huang, X. F.; Wallace, G. G. Electrical Stimulation with a Conductive Polymer Promotes Neurite Outgrowth and Synaptogenesis in Primary Cortical Neurons. Sci. Rep. 2018, 8, 9855.

(711) Simon, D. T.; Larsson, K. C.; Nilsson, D.; Burström, G.; Galter, D.; Berggren, M.; Richter-Dahlfors, A. An Organic Electronic Biomimetic Neuron Enables Auto-Regulated Neuromodulation. Biosens. Bioelectron. 2015, 71, 359-364.

(712) Cho, Y.; Shi, R.; Ivanisevic, A.; Ben Borgens, R. A Mesoporous Silica Nanosphere-Based Drug Delivery System Using an Electrically Conducting Polymer. Nanotechnology 2009, 20, 275102.

(713) Boyden, E. S. A History of Optogenetics: The Development of Tools for Controlling Brain Circuits with Light. F1000 Biol. Rep. 2011, 3,11 .

(714) Matarèse, B. F. E.; Feyen, P. L. C.; de Mello, J. C.; Benfenati, F. Sub-Millisecond Control of Neuronal Firing by Organic Light-Emitting Diodes. Front. Bioeng. Biotechnol. 2019, 7, 278.

(715) Morton, A.; Murawski, C.; Pulver, S. R.; Gather, M. C. HighBrightness Organic Light-Emitting Diodes for Optogenetic Control of Drosophila Locomotor Behaviour. Sci. Rep. 2016, 6, 31117.

(716) Sridharan, A.; Shah, A.; Kumar, S. S.; Kyeh, J.; Smith, J.; BlainChristen, J.; Muthuswamy, J. Optogenetic Modulation of Cortical Neurons Using Organic Light Emitting Diodes (OLEDs). Biomed. Phys. Eng. Express 2020, 6, 025003.

(717) Abdullaeva, O. S.; Schulz, M.; Balzer, F.; Parisi, J.; Lützen, A.; Dedek, K.; Schiek, M. Photoelectrical Stimulation of Neuronal Cells by 
an Organic Semiconductor-Electrolyte Interface. Langmuir 2016, 32, $8533-8542$.

(718) Sherwood, C.; Elkington, D. C.; Dickinson, M.; Belcher, W.; Dastoor, P. C.; Feron, K.; Brichta, A.; Lim, R.; Griffith, M. J. Organic Semiconductors for Optically Triggered Neural Interfacing: The Impact of Device Architecture in Determining Response Magnitude and Polarity. IEEE J. Sel. Top. Quantum Electron. 2021, 27, 7400212.

(719) DiFrancesco, M. L.; Colombo, E.; Papaleo, E. D.; Maya Vetencourt, J. F.; Manfredi, G.; Lanzani, G.; Benfenati, F. A Hybrid P3HT-Graphene Interface for Efficient Photostimulation of Neurons. Carbon 2020, 162, 308-317.

(720) Feyen, P.; Colombo, E.; Endeman, D.; Nova, M.; Laudato, L.; Martino, N.; Antognazza, M. R.; Lanzani, G.; Benfenati, F.; Ghezzi, D. Light-Evoked Hyperpolarization and Silencing of Neurons by Conjugated Polymers. Sci. Rep. 2016, 6, 22718.

(721) Hsiao, Y. S.; Liao, Y. H.; Chen, H. L.; Chen, P.; Chen, F. C. Organic Photovoltaics and Bioelectrodes Providing Electrical Stimulation for PC12 Cell Differentiation and Neurite Outgrowth. ACS Appl. Mater. Interfaces 2016, 8, 9275-9284.

(722) Wu, Y.; Peng, Y.; Bohra, H.; Zou, J.; Ranjan, V. D.; Zhang, Y.; Zhang, Q.; Wang, M. Photoconductive Micro/Nanoscale Interfaces of a Semiconducting Polymer for Wireless Stimulation of Neuron-Like Cells. ACS Appl. Mater. Interfaces 2019, 11, 4833-4841.

(723) Fernandes, E. G. R.; Zucolotto, V.; De Queiroz, A. A. A. Electrospinning of Hyperbranched Poly-L-Lysine/Polyaniline Nanofibers for Application in Cardiac Tissue Engineering. J. Macromol. Sci., Part A: Pure Appl.Chem. 2010, 47, 1203-1207.

(724) Kai, D.; Prabhakaran, M. P.; Jin, G.; Ramakrishna, S. Biocompatibility Evaluation of Electrically Conductive Nanofibrous Scaffolds for Cardiac Tissue Engineering. J. Mater. Chem. B 2013, 1, $2305-2314$.

(725) Roshanbinfar, K.; Vogt, L.; Greber, B.; Diecke, S.; Boccaccini, A. R.; Scheibel, T.; Engel, F. B. Electroconductive Biohybrid Hydrogel for Enhanced Maturation and Beating Properties of Engineered Cardiac Tissues. Adv. Funct. Mater. 2018, 28, 1803951.

(726) Nishizawa, M.; Nozaki, H.; Kaji, H.; Kitazume, T.; Kobayashi, N.; Ishibashi, T.; Abe, T. Electrodeposition of Anchored Polypyrrole Film on Microelectrodes and Stimulation of Cultured Cardiac Myocytes. Biomaterials 2007, 28, 1480-1485.

(727) Hsiao, C. W.; Bai, M. Y.; Chang, Y.; Chung, M. F.; Lee, T. Y.; Wu, C. T.; Maiti, B.; Liao, Z. X.; Li, R. K.; Sung, H. W. Electrical Coupling of Isolated Cardiomyocyte Clusters Grown on Aligned Conductive Nanofibrous Meshes for Their Synchronized Beating. Biomaterials 2013, 34, 1063-1072.

(728) Thourson, S. B.; Payne, C. K. Modulation of Action Potentials Using PEDOT: PSS Conducting Polymer Microwires. Sci. Rep. 2017, 7, 10402 .

(729) Hoop, M.; Chen, X. Z.; Ferrari, A.; Mushtaq, F.; Ghazaryan, G.; Tervoort, T.; Poulikakos, D.; Nelson, B.; Pané, S. Ultrasound-Mediated Piezoelectric Differentiation of Neuron-like PC12 Cells on PVDF Membranes. Sci. Rep. 2017, 7, 4028.

(730) Lee, Y. S.; Collins, G.; Livingston Arinzeh, T. Neurite Extension of Primary Neurons on Electrospun Piezoelectric Scaffolds. Acta Biomater. 2011, 7, 3877-3886.

(731) Royo-Gascon, N.; Wininger, M.; Scheinbeim, J. I.; Firestein, B. L.; Craelius, W. Piezoelectric Substrates Promote Neurite Growth in Rat Spinal Cord Neurons. Ann. Biomed. Eng. 2013, 41, 112-122.

(732) Hitscherich, P.; Wu, S.; Gordan, R.; Xie, L.-H.; Arinzeh, T.; Lee, E. J. The Effect of PVDF-TrFE Scaffolds on Stem Cell Derived Cardiovascular Cells. Biotechnol. Bioeng. 2016, 113, 1577-1585.

(733) Liu, Y.; McGuire, A. F.; Lou, H. Y.; Li, T. L.; Tok, J. B. H.; Cui, B.; Bao, Z. Soft Conductive Micropillar Electrode Arrays for Biologically Relevant Electrophysiological Recording. Proc. Natl. Acad. Sci. U. S. A. 2018, 115, 11718-11723.

(734) Liu, X.; Zhao, H.; Lu, Y.; Li, S.; Lin, L.; Du, Y.; Wang, X. In Vitro Cardiomyocyte-Driven Biogenerator Based on Aligned Piezoelectric Nanofibers. Nanoscale 2016, 8, 7278-7286.
(735) Boys, A. J.; Owens, R. M. Measuring Cellular Contraction: Current Progress and a Future in Bioelectronics. APL Mater. 2021, 9, 040903.

(736) Higuchi, A.; Suresh Kumar, S.; Ling, Q.-D.; Alarfaj, A. A.; Munusamy, M. A.; Murugan, K.; Hsu, S.-T.; Benelli, G.; Umezawa, A. Polymeric Design of Cell Culture Materials That Guide the Differentiation of Human Pluripotent Stem Cells. Prog. Polym. Sci. 2017, 65, 83-126.

(737) Mahla, R. S. Stem Cells Applications in Regenerative Medicine and Disease Therapeutics. Int. J. Cell Biol. 2016, 2016, 6940283.

(738) Tomaskovic-Crook, E.; Higginbottom, S. L.; James, E. C.; Rathbone, S. J. C.; Crook, J. M. Electroceuticals for Neural Regenerative Nanomedicine; In Neural Regenerative Nanomedicine; Razavi, M., Ed.; Academic Press, 2020; Chapter 7, pp 213-257.

(739) Levin, M.; Pezzulo, G.; Finkelstein, J. M. Endogenous Bioelectric Signaling Networks: Exploiting Voltage Gradients for Control of Growth and Form. Annu. Rev. Biomed. Eng. 2017, 19, 353-387.

(740) Stewart, E.; Kobayashi, N. R.; Higgins, M. J.; Quigley, A. F.; Jamali, S.; Moulton, S. E.; Kapsa, R. M. I.; Wallace, G. G.; Crook, J. M. Electrical Stimulation Using Conductive Polymer Polypyrrole Promotes Differentiation of Human Neural Stem Cells: A Biocompatible Platform for Translational Neural Tissue Engineering. Tissue Eng., Part C 2015, 21, 385-393.

(741) Ritzau-Reid, K. I.; Spicer, C. D.; Gelmi, A.; Grigsby, C. L.; Ponder, J. F.; Bemmer, V.; Creamer, A.; Vilar, R.; Serio, A.; Stevens, M. M. An Electroactive Oligo-EDOT Platform for Neural Tissue Engineering. Adv. Funct. Mater. 2020, 30, 2003710.

(742) Heo, D. N.; Acquah, N.; Kim, J.; Lee, S. J.; Castro, N. J.; Zhang, L. G. Directly Induced Neural Differentiation of Human AdiposeDerived Stem Cells Using Three-Dimensional Culture System of Conductive Microwell with Electrical Stimulation. Tissue Eng., Part A 2018, 24, 537-545.

(743) Fukada, E.; Yasuda, I. On the Piezoelectric Effect of Bone. J. Phys. Soc. Jpn. 1957, 12, 1158-1162.

(744) Iandolo, D.; Sheard, J.; Karavitas Levy, G.; Pitsalidis, C.; Tan, E.; Dennis, A.; Kim, J. S.; Markaki, A. E.; Widera, D.; Owens, R. M. Biomimetic and Electroactive 3D Scaffolds for Human Neural CrestDerived Stem Cell Expansion and Osteogenic Differentiation. MRS Commun. 2020, 10, 179-187.

(745) Hardy, J. G.; Sukhavasi, R. C.; Aguilar, D.; Villancio-Wolter, M. K.; Mouser, D. J.; Geissler, S. A.; Nguy, L.; Chow, J. K.; Kaplan, D. L.; Schmidt, C. E. Electrical Stimulation of Human Mesenchymal Stem Cells on Biomineralized Conducting Polymers Enhances Their Differentiation towards Osteogenic Outcomes. J. Mater. Chem. B 2015, 3, 8059-8064.

(746) He, Y.; Wang, S.; Mu, J.; Dai, L.; Zhang, Z.; Sun, Y.; Shi, W.; Ge, D. Synthesis of Polypyrrole Nanowires with Positive Effect on MC3T3E1 Cell Functions through Electrical Stimulation. Mater. Sci. Eng., C 2017, 71, 43-50.

(747) Meng, S.; Zhang, Z.; Rouabhia, M. Accelerated Osteoblast Mineralization on a Conductive Substrate by Multiple Electrical Stimulation. J. Bone Miner. Metab. 2011, 29, 535-544.

(748) Zhang, J.; Li, M.; Kang, E. T.; Neoh, K. G. Electrical Stimulation of Adipose-Derived Mesenchymal Stem Cells in Conductive Scaffolds and the Roles of Voltage-Gated Ion Channels. Acta Biomater. 2016, 32, $46-56$.

(749) Zhang, J.; Neoh, K. G.; Kang, E. T. Electrical Stimulation of Adipose-Derived Mesenchymal Stem Cells and Endothelial Cells CoCultured in a Conductive Scaffold for Potential Orthopaedic Applications. J. Tissue Eng. Regener. Med. 2018, 12, 878-889.

(750) Wei, Y.; Mo, X.; Zhang, P.; Li, Y.; Liao, J.; Li, Y.; Zhang, J.; Ning, C.; Wang, S.; Deng, X.; et al. Directing Stem Cell Differentiation via Electrochemical Reversible Switching between Nanotubes and Nanotips of Polypyrrole Array. ACS Nano 2017, 11, 5915-5924.

(751) Cheng, Y. C.; Chen, C. H.; Kuo, H. W.; Yen, T. L.; Mao, Y. Y.; $\mathrm{Hu}, \mathrm{W}$. W. Electrical Stimulation through Conductive Substrate to Enhance Osteo-Differentiation of Human Dental Pulp-Derived Stem Cells. Appl. Sci. 2019, 9, 3938. 
(752) Cui, L.; Zhang, J.; Zou, J.; Yang, X.; Guo, H.; Tian, H.; Zhang, P.; Wang, Y.; Zhang, N.; Zhuang, X.; et al. Electroactive Composite Scaffold with Locally Expressed Osteoinductive Factor for Synergistic Bone Repair upon Electrical Stimulation. Biomaterials 2020, 230, 119617.

(753) McConnell, H. M.; Owicki, J. C.; Parce, J. W.; Miller, D. L.; Baxter, G. T.; Wada, H. G.; Pitchford, S. The Cytosensor Microphysiometer: Biological Applications of Silicon Technology. Science 1992, 257, 1906-1912.

(754) Kieninger, J.; Weltin, A.; Flamm, H.; Urban, G. A. Microsensor Systems for Cell Metabolism-from 2D Culture to Organ-on-Chip. Lab Chip 2018, 18, 1274-1291.

(755) Modena, M. M.; Chawla, K.; Misun, P. M.; Hierlemann, A. Smart Cell Culture Systems: Integration of Sensors and Actuators into Microphysiological Systems. ACS Chem. Biol. 2018, 13, 1767-1784.

(756) Turner, A. P. F. Biosensors: Sense and Sensibility. Chem. Soc. Rev. 2013, 42, 3184.

(757) Derkus, B. Applying the Miniaturization Technologies for Biosensor Design. Biosens. Bioelectron. 2016, 79, 901-913.

(758) Shinawi, T. F.; Kimmel, D. W.; Cliffel, D. E. Multianalyte Microphysiometry Reveals Changes in Cellular Bioenergetics upon Exposure to Fluorescent Dyes. Anal. Chem. 2013, 85, 11677-11680.

(759) Ntziachristos, V.; Pleitez, M. A.; Aime, S.; Brindle, K. M. Emerging Technologies to Image Tissue Metabolism. Cell Metab. 2019, 29, 518-538.

(760) Müller, J.; Ballini, M.; Livi, P.; Chen, Y.; Radivojevic, M.; Shadmani, A.; Viswam, V.; Jones, I. L.; Fiscella, M.; Diggelmann, R.; et al. High-Resolution CMOS MEA Platform to Study Neurons at Subcellular, Cellular, and Network Levels. Lab Chip 2015, 15, 27672780.

(761) Hierlemann, A.; Frey, U.; Hafizovic, S.; Heer, F. Growing Cells Atop Microelectronic Chips: Interfacing Electrogenic Cells In Vitro With CMOS-Based Microelectrode Arrays. Proc. IEEE 2011, 99, 252284.

(762) Ronkainen, N. J.; Halsall, H. B.; Heineman, W. R. Electrochemical Biosensors. Chem. Soc. Rev. 2010, 39, 1747-1763.

(763) Ges, I. A.; Baudenbacher, F. Enzyme Electrodes to Monitor Glucose Consumption of Single Cardiac Myocytes in Sub-Nanoliter Volumes. Biosens. Bioelectron. 2010, 25, 1019-1024.

(764) Piro, B.; Mattana, G.; Reisberg, S. Transistors for Chemical Monitoring of Living Cells. Biosensors 2018, 8, 65.

(765) Rivnay, J.; Owens, R. M.; Malliaras, G. G. The Rise of Organic Bioelectronics. Chem. Mater. 2014, 26, 679-685.

(766) Pitsalidis, C.; Pappa, A. M.; Moysidou, C. M.; Iandolo, D.; Owens, R. M. Conducting and Conjugated Polymers for Biosensing Applications. Conjug. Polym. 2019, 697-742.

(767) Adhikari, B.; Majumdar, S. Polymers in Sensor Applications. Prog. Polym. Sci. 2004, 29, 699-766.

(768) Forzani, E. S.; Zhang, H.; Nagahara, L. A.; Amlani, I.; Tsui, R.; Tao, N. A Conducting Polymer Nanojunction Sensor for Glucose Detection. Nano Lett. 2004, 4, 2519.

(769) Khan, R.; Solanki, P. R.; Kaushik, A.; Singh, S. P.; Ahmad, S.; Malhotra, B. D. Cholesterol Biosensor Based on Electrochemically Prepared Polyaniline Conducting Polymer Film in Presence of a Nonionic Surfactant. J. Polym. Res. 2009, 16, 363-373.

(770) Kumar, K.; Tang, C. S.; Rossetti, F. F.; Textor, M.; Keller, B.; Vörös, J.; Reimhult, E. Formation of Supported Lipid Bilayers on Indium Tin Oxide for Dynamically-Patterned Membrane-Functionalized Microelectrode Arrays. Lab Chip 2009, 9, 718-725.

(771) Wustoni, S.; Wang, S.; Alvarez, J. R.; Hidalgo, T. C.; Nunes, S. P.; Inal, S. An Organic Electrochemical Transistor Integrated with a Molecularly Selective Isoporous Membrane for Amyloid- $\beta$ Detection. Biosens. Bioelectron. 2019, 143, 111561.

(772) Wang, J. Electrochemical Glucose Biosensors. Chem. Rev. 2008, $108,814-825$.

(773) Strakosas, X.; Huerta, M.; Donahue, M. J.; Hama, A.; Pappa, A.M.; Ferro, M.; Ramuz, M.; Rivnay, J.; Owens, R. M. Catalytically Enhanced Organic Transistors for in Vitro Toxicology Monitoring through Hydrogel Entrapment of Enzymes. J. Appl. Polym. Sci. 2017, 134,44483 .

(774) Cass, A. E. G.; Davis, G.; Francis, G. D.; Hill, H. A. O.; Aston, W. J.; Higgins, I. J.; Plotkin, E. V.; Scott, L. D. L.; Turner, A. P. F. Ferrocene-Mediated Enzyme Electrode for Amperometric Determination of Glucose. Anal. Chem. 1984, 56, 667-671.

(775) Scheiblin, G.; Aliane, A.; Strakosas, X.; Curto, V. F.; Coppard, R.; Marchand, G.; Owens, R. M.; Mailley, P.; Malliaras, G. G. ScreenPrinted Organic Electrochemical Transistors for Metabolite Sensing. MRS Commun. 2015, 5, 507-511.

(776) Pappa, A.-M.; Curto, V. F.; Braendlein, M.; Strakosas, X.; Donahue, M. J.; Fiocchi, M.; Malliaras, G. G.; Owens, R. M. Organic Transistor Arrays Integrated with Finger-Powered Microfluidics for Multianalyte Saliva Testing. Adv. Healthcare Mater. 2016, 5, 22952302.

(777) Braendlein, M.; Pappa, A. M.; Ferro, M.; Lopresti, A.; Acquaviva, C.; Mamessier, E.; Malliaras, G. G.; Owens, R. M. Lactate Detection in Tumor Cell Cultures Using Organic Transistor Circuits. Adv. Mater. 2017, 29, 1605744.

(778) Park, S. J.; Lee, J.; Seo, S. E.; Kim, K. H.; Park, C. S.; Lee, S. H.; Ban, H. S.; Lee, B. D.; Song, H. S.; Kim, J.; et al. High-Performance Conducting Polymer Nanotube-Based Liquid-Ion Gated Field-Effect Transistor Aptasensor for Dopamine Exocytosis. Sci. Rep. 2020, 10, 3772.

(779) Larsen, S. T.; Vreeland, R. F.; Heien, M. L.; Taboryski, R. Characterization of Poly(3,4-Ethylenedioxythiophene):Tosylate Conductive Polymer Microelectrodes for Transmitter Detection. Analyst 2012, 137, 1831-1836.

(780) Yang, S. Y.; Kim, B. N.; Zakhidov, A. A.; Taylor, P. G.; Lee, J. K.; Ober, C. K.; Lindau, M.; Malliaras, G. G. Detection of Transmitter Release from Single Living Cells Using Conducting Polymer Microelectrodes. Adv. Mater. 2011, 23, H184.

(781) Sessolo, M.; Rivnay, J.; Bandiello, E.; Malliaras, G. G.; Bolink, H. J. Ion-Selective Organic Electrochemical Transistors. Adv. Mater. 2014, 26, 4803-4807.

(782) Svarovsky, S. A.; Joshi, L. Cancer Glycan Biomarkers and Their Detection-Past, Present and Future. Anal. Methods 2014, 6, 39183936.

(783) Wang, N.; Liu, Y.; Fu, Y.; Yan, F. AC Measurements Using Organic Electrochemical Transistors for Accurate Sensing. ACS Appl. Mater. Interfaces 2018, 10, 25834.

(784) Lin, P.; Yan, F. Organic Thin-Film Transistors for Chemical and Biological Sensing. Adv. Mater. 2012, 24, 34-51.

(785) Chen, L.; Fu, Y.; Wang, N.; Yang, A.; Li, Y.; Wu, J.; Ju, H.; Yan, F. Organic Electrochemical Transistors for the Detection of Cell Surface Glycans. ACS Appl. Mater. Interfaces 2018, 10, 18470-18477.

(786) Zhang, X.; Teng, Y.; Fu, Y.; Xu, L.; Zhang, S.; He, B.; Wang, C.; Zhang, W. Lectin-Based Biosensor Strategy for Electrochemical Assay of Glycan Expression on Living Cancer Cells. Anal. Chem. 2010, 82, 9455-9460.

(787) Chen, L.; Wu, J.; Yan, F.; Ju, H. A Facile Strategy for Quantitative Sensing of Glycans on Cell Surface Using Organic Electrochemical Transistors. Biosens. Bioelectron. 2021, 175, 112878.

(788) Chen, L.; Wang, N.; Wu, J.; Yan, F.; Ju, H. Organic Electrochemical Transistor for Sensing of Sialic Acid in Serum Samples. Anal. Chim. Acta 2020, 1128, 231-237.

(789) Fu, Y.; Wang, N.; Yang, A.; Law, H. K.-w.; Li, L.; Yan, F. Highly Sensitive Detection of Protein Biomarkers with Organic Electrochemical Transistors. Adv. Mater. 2017, 29, 1703787.

(790) Fu, Y.; Liu, K.; Sun, Q.; Lin, B.; Lu, D.; Xu, Z.; Hu, C.; Fan, G.; Zhang, S.; Wang, C.; et al. A Highly Sensitive Immunosensor for Calmodulin Assay Based on Enhanced Biocatalyzed Precipitation Adopting a Dual-Layered Enzyme Strategy. Biosens. Bioelectron. 2014, $56,258-263$.

(791) Elmore, S. Apoptosis: A Review of Programmed Cell Death. Toxicol. Pathol. 2007, 35, 495-516.

(792) Porter, A. G.; Jänicke, R. U. Emerging Roles of Caspase-3 in Apoptosis. Cell Death Differ. 1999, 6, 99-104. 
(793) Yu, J.; Yang, A.; Wang, N.; Ling, H.; Song, J.; Chen, X.; Lian, Y.; Zhang, Z.; Yan, F.; Gu, M. Highly Sensitive Detection of Caspase-3 Activity Based on Peptide-Modified Organic Electrochemical Transistor Biosensors. Nanoscale 2021, 13, 2868-2874.

(794) Thomas, R. S.; Morgan, H.; Green, N. G. Negative DEP Traps for Single Cell Immobilisation. Lab Chip 2009, 9, 1534-1540.

(795) Strakosas, X.; Donahue, M. J.; Hama, A.; Braendlein, M.; Huerta, M.; Simon, D. T.; Berggren, M.; Malliaras, G. G.; Owens, R. M. Biostack: Nontoxic Metabolite Detection from Live Tissue. Adv. Sci. 2021, 2101711.

(796) Cho, I.-H.; Kim, D. H.; Park, S. Electrochemical Biosensors: Perspective on Functional Nanomaterials for on-Site Analysis. Biomater. Res. 2020, 24, 6.

(797) Turner, A. P. F. Biosensors: Sense and Sensibility. Chem. Soc. Rev. 2013, 42, 3184.

(798) Jiang, C.; Alam, M. T.; Silva, S. M.; Taufik, S.; Fan, S.; Gooding, J. J. Unique Sensing Interface That Allows the Development of an Electrochemical Immunosensor for the Detection of Tumor Necrosis Factor $\alpha$ in Whole Blood. ACS Sensors 2016, 1, 1432-1438.

(799) Jiang, C.; Wang, G.; Hein, R.; Liu, N.; Luo, X.; Davis, J. J. Antifouling Strategies for Selective in Vitro and in Vivo Sensing. Chem. Rev. 2020, 120, 3852-3889.

(800) Fu, Y.; Jiang, C.; Tofaris, G. K.; Davis, J. J. Facile Impedimetric Analysis of Neuronal Exosome Markers in Parkinson's Disease Diagnostics. Anal. Chem. 2020, 92, 13647-13651.

(801) Goda, T.; Miyahara, Y. Electrodeposition of Zwitterionic PEDOT Films for Conducting and Antifouling Surfaces. Langmuir 2019, 35, 1126-1133.

(802) Wang, W.; Cui, M.; Song, Z.; Luo, X. An Antifouling Electrochemical Immunosensor for Carcinoembryonic Antigen Based on Hyaluronic Acid Doped Conducting Polymer PEDOT. RSC Adv. 2016, 6, 88411-88416.

(803) Pappa, A.; Curto, V. F.; Braendlein, M.; Strakosas, X.; Donahue, M. J.; Fiocchi, M.; Malliaras, G. G.; Owens, R. M. Organic Transistor Arrays Integrated with Finger-Powered Microfluidics for Multianalyte Saliva Testing. Adv. Healthcare Mater. 2016, 5, 2295.

(804) Tan, E.; Pappa, A.-M.; Pitsalidis, C.; Nightingale, J.; Wood, S.; Castro, F. A.; Owens, R. M.; Kim, J.-S. A Highly Sensitive Molecular Structural Probe Applied to in Situ Biosensing of Metabolites Using PEDOT:PSS. Biotechnol. Bioeng. 2020, 117, 291.

(805) Tan, S. T. M.; Giovannitti, A.; Melianas, A.; Moser, M.; Cotts, B. L.; Singh, D.; McCulloch, I.; Salleo, A. High-Gain Chemically Gated Organic Electrochemical Transistor. Adv. Funct. Mater. 2021, 31, 2010868.

(806) Ramadan, Q.; Zourob, M. Organ-on-a-Chip Engineering: Toward Bridging the Gap between Lab and Industry. Biomicrofluidics 2020, 14, 041501.

(807) Sackmann, E. K.; Fulton, A. L.; Beebe, D. J. The Present and Future Role of Microfluidics in Biomedical Research. Nature 2014, 507, 181-189.

(808) Saez, J.; Glennon, T.; Czugala, M.; Tudor, A.; Ducreé, J.; Diamond, D.; Florea, L.; Benito-Lopez, F. Reusable Ionogel-Based Photo-Actuators in a Lab-on-a-Disc. Sens. Actuators, B 2018, 257, $963-$ 970.

(809) Tudor, A.; Saez, J.; Florea, L.; Benito-Lopez, F.; Diamond, D. Poly(Ionic Liquid) Thermo-Responsive Hydrogel Microfluidic Actuators. Sens. Actuators, B 2017, 247, 749-755.

(810) Henry, O. Y. F.; Villenave, R.; Cronce, M. J.; Leineweber, W. D.; Benz, M. A.; Ingber, D. E. Organs-on-Chips with Integrated Electrodes for Trans-Epithelial Electrical Resistance (TEER) Measurements of Human Epithelial Barrier Function. Lab Chip 2017, 17, 2264-2271.

(811) Saez, J.; Etxebarria, J.; Antoñana-Diez, M.; Benito-Lopez, F. OnDemand Generation and Removal of Alginate Biocompatible Microvalves for Flow Control in Microfluidics. Sens. Actuators, B 2016, 234, $1-7$.

(812) Lanfranco, R.; Saez, J.; Di Nicolò, E.; Benito-Lopez, F.; Buscaglia, M. Phantom Membrane Microfluidic Cross-Flow Filtration Device for the Direct Optical Detection of Water Pollutants. Sens. Actuators, B 2018, 257, 924-930.
(813) Saez, J.; Basabe-Desmonts, L.; Benito-Lopez, F. Low-Cost Origami Fabrication of 3D Self-Aligned Hybrid Microfluidic Structures. Microfluid. Nanofluid. 2016, 20, 116.

(814) Hou, X.; Zhang, Y. S.; Santiago, G. T. De; Alvarez, M. M.; Ribas, J.; Jonas, S. J.; Weiss, P. S.; Andrews, A. M.; Aizenberg, J.; Khademhosseini, A. Interplay between Materials and Microfluidics. Nat. Rev. Mater. 2017, 2, 17016.

(815) Campbell, S. B.; Wu, Q.; Yazbeck, J.; Liu, C.; Okhovatian, S.; Radisic, M. Beyond Polydimethylsiloxane: Alternative Materials for Fabrication of Organ-on-a-Chip Devices and Microphysiological Systems. ACS Biomater. Sci. Eng. 2021, 7, 2880.

(816) Sung, J. H.; Kam, C.; Shuler, M. L. A Microfluidic Device for a Pharmacokinetic-Pharmacodynamic (PK-PD) Model on a Chip. Lab Chip 2010, 10, 446-455.

(817) Baker, M. Tissue Models: A Living System on a Chip. Nature 2011, 471, 661-665.

(818) Mi, S.; Xia, J.; Xu, Y.; Du, Z.; Sun, W. An Integrated Microchannel Biosensor Platform to Analyse Low Density Lactate Metabolism in HepG2 Cells in Vitro. RSC Adv. 2019, 9, 9006.

(819) Hur, W.; Son, S. E.; Seong, G. H. Electrochemical Live Cell Patterning. Electrochem. Commun. 2020, 117, 106778.

(820) Koklu, A.; Wustoni, S.; Musteata, V.-E.; Ohayon, D.; Moser, M.; McCulloch, I.; Nunes, S. P.; Inal, S. Microfluidic Integrated Organic Electrochemical Transistor with a Nanoporous Membrane for Amyloid- $\beta$ Detection. ACS Nano 2021, 15, 8130.

(821) Uguz, I.; Proctor, C. M.; Curto, V. F.; Pappa, A.-M.; Donahue, M. J.; Ferro, M.; Owens, R. M.; Khodagholy, D.; Inal, S.; Malliaras, G. G. A Microfluidic Ion Pump for In Vivo Drug Delivery. Adv. Mater. 2017, 29, 1701217.

(822) Liu, Z.; Jin, M.; Cao, J.; Niu, R.; Li, P.; Zhou, G.; Yu, Y.; van den Berg, A.; Shui, L. Electrochemical Sensor Integrated Microfluidic Device for Sensitive and Simultaneous Quantification of Dopamine and 5-Hydroxytryptamine. Sens. Actuators, B 2018, 273, 873-883.

(823) Naskar, S.; Panda, A. K.; Kumaran, V.; Mehta, B.; Basu, B. Controlled Shear Flow Directs Osteogenesis on UHMWPE-Based Hybrid Nanobiocomposites in a Custom-Designed PMMA Microfluidic Device. ACS Appl. Bio Mater. 2018, 1, 414.

(824) Li, L.; Chen, Z.; Shao, C.; Sun, L.; Sun, L.; Zhao, Y. Graphene Hybrid Anisotropic Structural Color Film for Cardiomyocytes' Monitoring. Adv. Funct. Mater. 2020, 30, 1906353.

(825) Sakai, K.; Shimba, K.; Kotani, K.; Jimbo, Y. A Co-Culture Microtunnel Technique Demonstrating a Significant Contribution of Unmyelinated Schwann Cells to the Acceleration of Axonal Conduction in Schwann Cell-Regulated Peripheral Nerve Development $\dagger$. Integr. Biol. 2017, 9, 678.

(826) Kanagasabapathi, T. T.; Ciliberti, D.; Martinoia, S.; Wadman, W. J.; Decré, M. M. J. Dual-Compartment Neurofluidic System for Electrophysiological Measurements in Physically Segregated and Functionally Connected Neuronal Cell Culture. Front. Neuroeng. $2011,4,13$.

(827) Kanagasabapathi, T. T.; Massobrio, P.; Barone, R. A.; Tedesco, M.; Martinoia, S.; Wadman, W. J.; Decré, M. M. J. Functional Connectivity and Dynamics of Cortical-Thalamic Networks CoCultured in a Dual Compartment Device. J. Neural Eng. 2012, 9, 036010.

(828) Tarabella, G.; Balducci, A. G.; Coppedè, N.; Marasso, S.; D’Angelo, P.; Barbieri, S.; Cocuzza, M.; Colombo, P.; Sonvico, F.; Mosca, R.; et al. Liposome Sensing and Monitoring by Organic Electrochemical Transistors Integrated in Microfluidics. Biochim. Biophys. Acta, Gen. Subj. 2013, 1830, 4374-4380.

(829) Yu, C. C.; Ho, B. C.; Juang, R. S.; Hsiao, Y. S.; Naidu, R. V. R.; Kuo, C. W.; You, Y. W.; Shyue, J. J.; Fang, J. T.; Chen, P. Poly(3,4Ethylenedioxythiophene)-Based Nanofiber Mats as an Organic Bioelectronic Platform for Programming Multiple Capture/Release Cycles of Circulating Tumor Cells. ACS Appl. Mater. Interfaces 2017, 9, 30329-30342.

(830) Ji, J.; Li, M.; Chen, Z.; Wang, H.; Jiang, X.; Zhuo, K.; Liu, Y.; Yang, X.; Gu, Z.; Sang, S.; Shu, Y. In Situ Fabrication of Organic 
Electrochemical Transistors on a Microfluidic Chip. Nano Res. 2019, 12, 1943.

(831) Jin, Z.; Liu, Y.; Fan, W.; Huang, W. Integrating Flexible Electrochemical Sensor into Microfluidic Chip for Simulating and Monitoring Vascular Mechanotransduction. Small 2020, 16, 1903204. 